\title{
TESTING REYCLED CONCRETE AGGREGATE SUFFERING DIFFERENT LEVELS OF ALKALI-SILICA REACTION FOR USE IN NEW STRUCTURES
}

by

Matthew Piersanti, B.Eng

Ryerson University, 2013

\author{
A thesis \\ presented to Ryerson University \\ in partial fulfillment of the \\ requirements for the degree of \\ Master of Applied Science \\ in the Program of \\ Civil Engineering
}

Toronto, Ontario, Canada, 2015

(C) Matthew Piersanti 2015 


\section{Author's Declaration}

I hereby declare that I am the sole author of this thesis. This is a true copy of the thesis, including any required final revisions, as accepted by my examiners.

I authorize Ryerson University to lend this thesis to other institutions or individuals for the purpose of scholarly research.

I further authorize Ryerson University to reproduce this thesis by photocopying or by other means, in total or in part, at the request of other institutions or individuals for the purpose of scholarly research.

I understand that my thesis may be made electronically available to the public. 


\begin{abstract}
TESTING REYCLED CONCRETE AGGREGATE SUFFERING DIFFERENT LEVELS OF

ALKALI-SILICA REACTION FOR USE IN NEW STRUCTURES
\end{abstract}

Master of Applied Science, 2015

Matthew Piersanti

Department of Civil Engineering

Ryerson University

As concrete reaches the end of its service life, it is demolished and placed in landfills, which is not sustainable as this consumes land space. Many demolished structures are crushed into recycled concrete aggregate (RCA) and used in new construction work to reduce concrete waste. To be used in concrete, the effects of RCA on the new structures should be carefully examined. The RCA studied in this research is an alkali-silica reactive gravel from Sudbury, Ontario. The RCA was obtained from different elements of a 20-year old bridge that suffered different levels of deterioration. It was determined that the level of deterioration that affected the previous structure does not significantly affect the expansion that will occur in the new structure. It was also determined that the expansion could be mitigated through the use of supplementary cementing materials although higher levels are required compared those required for the virgin aggregate. 


\section{Acknowledgements}

I would like to thank my supervisor, Dr. Medhat Shehata, for providing me with the opportunity to complete the research presented in this paper. His continuous support, guidance, and dedication for his students is incomparable and has motivated me to pursue this degree.

I would also like to thank the Ontario Ministry of Transportation (MTO) as this research was funded by a grant under the Highway Infrastructure Innovation Funding Program. In addition, the help and support through the duration of this research from Carole Anne MacDonald and Stephen Senior from the MTO has not gone unnoticed.

In addition, I would like to thank my family for providing me the opportunity to further my education. Without their support and encouragement, it would not have been possible.

Finally, I would like to thank my fellow members of Dr. Shehata's research team for your help and support in the lab. 


\section{Table of Contents}

Author's Declaration.............................................................. ii

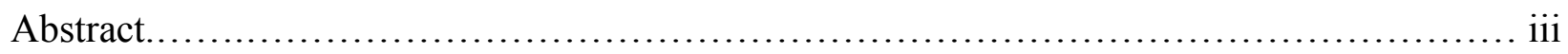

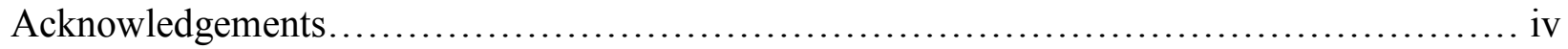

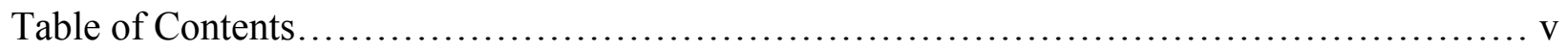

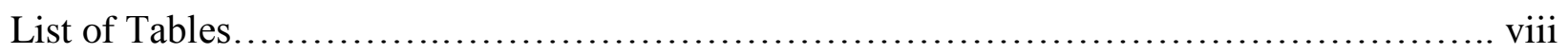

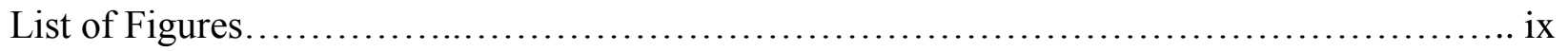

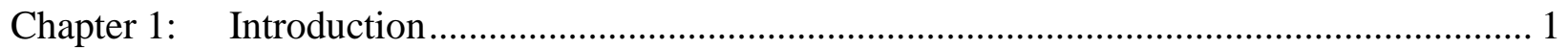

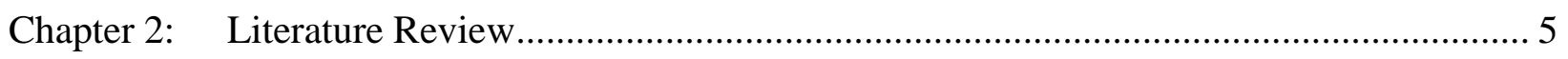

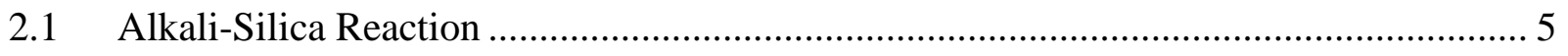

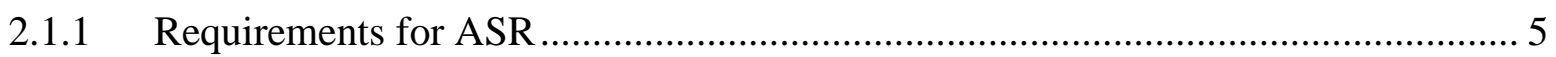

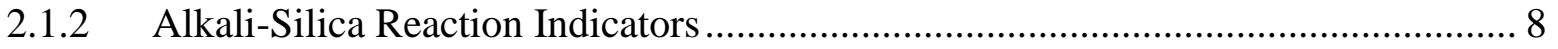

2.1.3 Alkali-Silica Reaction Lab Testing Methods................................................. 13

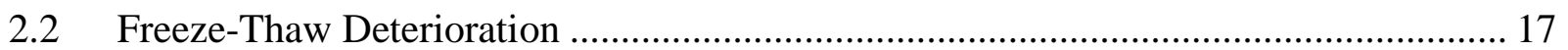

2.2.1 Causes for Freeze-Thaw Deterioration .......................................................... 18

2.2.2 Indicators for Freeze-Thaw Deterioration ..................................................... 22

2.2.3 Freeze-Thaw Deterioration Lab Testing Methods ............................................ 25

2.3 Supplementary Cementing Materials ................................................................. 28

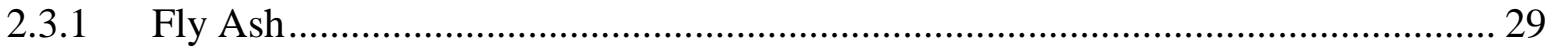

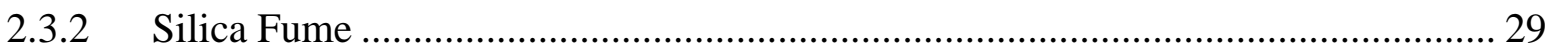

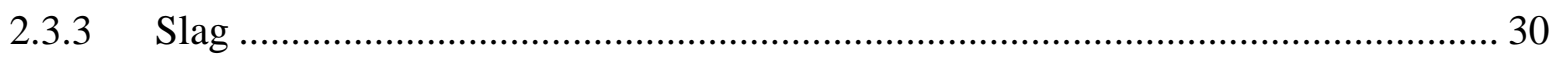

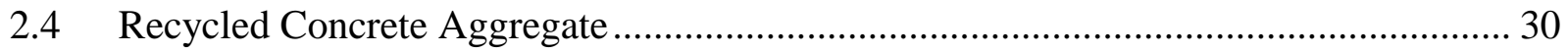

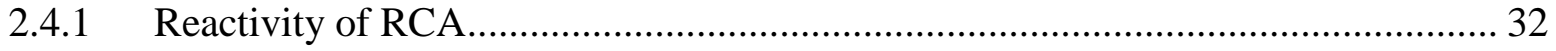

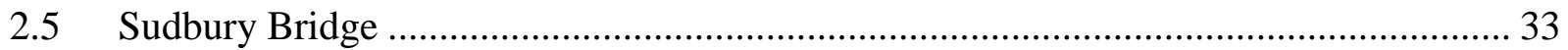

2.4.1 Bridge Condition Surveys ........................................................................... 35

Chapter 3: Experimental Procedures and Material Details................................................ 37

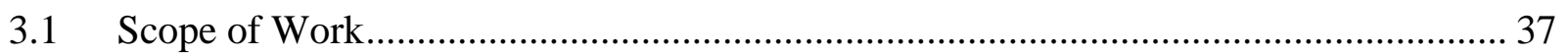

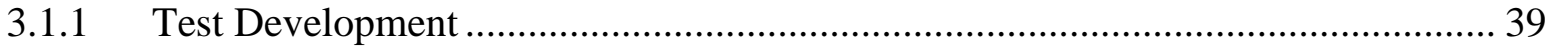

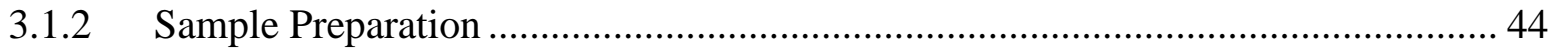

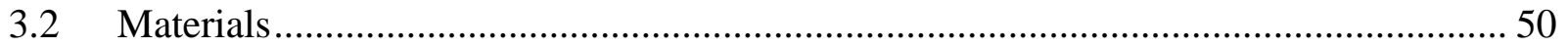

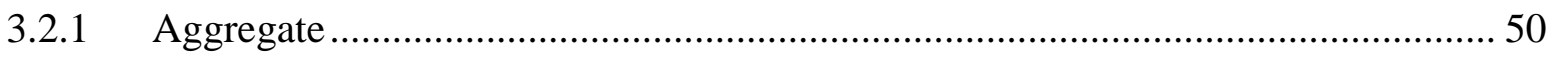

3.2.2 Cementing Materials .............................................................................. 52 


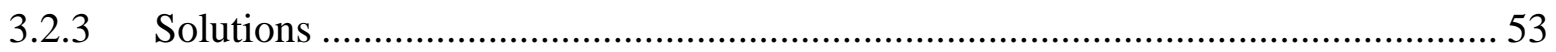

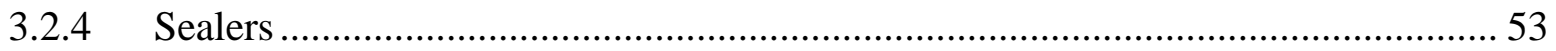

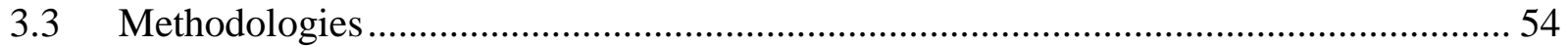

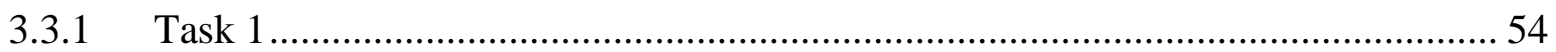

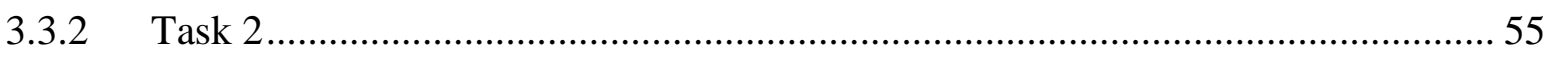

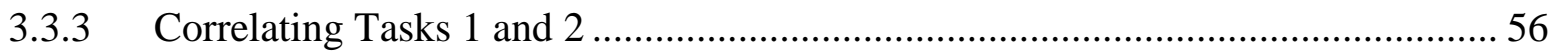

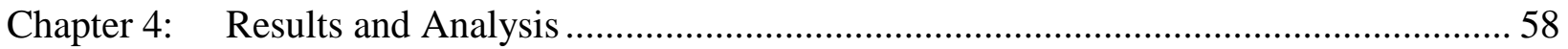

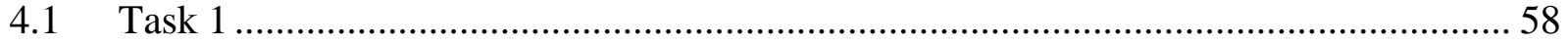

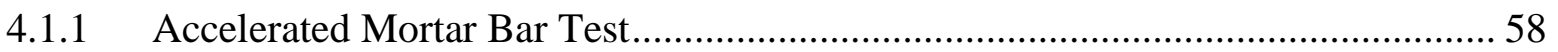

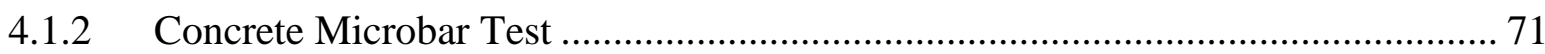

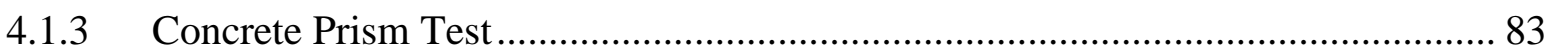

4.1.4 Comparing the AMBT and CPT Results .............................................................. 93

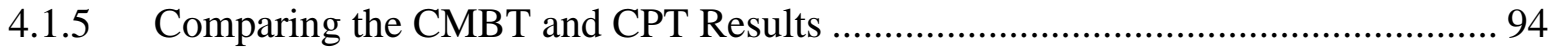

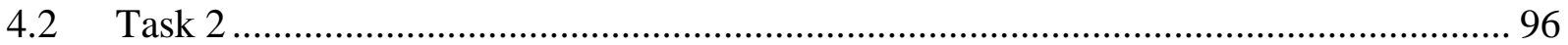

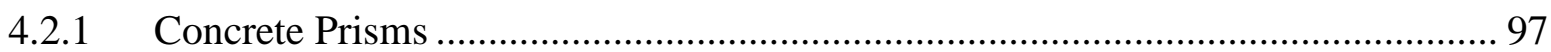

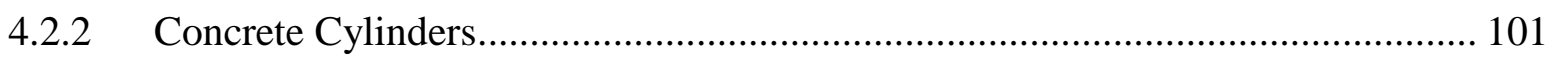

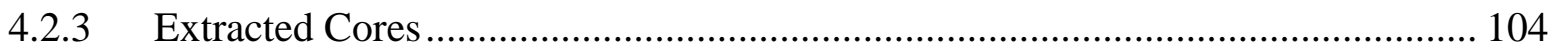

4.2.4 Comparing Lab Data: Prisms, Cylinders, and Cores ............................................ 105

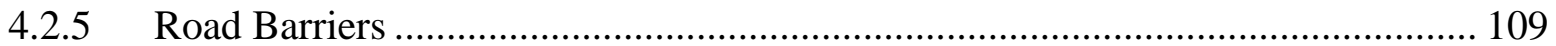

4.2.6 Comparing Lab and Field Data...................................................................... 110

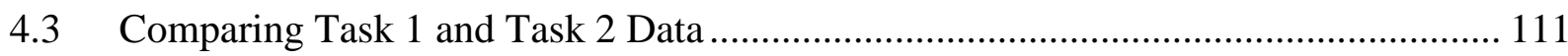

4.3.1 Comparing RCA-M, RCA-H, and RCA-L ………........................................ 112

4.3.2 Comparing the Effects of SCM and the Silane-based Sealer ............................. 112

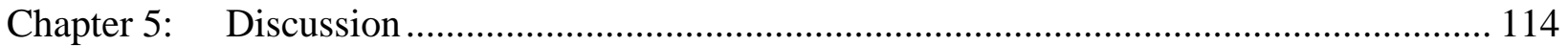

5.1 Analyzing the Effects of SCM on Sudbury Aggregates ........................................... 114

5.2 Analyzing the Expansions of Virgin Sudbury aggregate and Sudbury RCA-M.......... 116

5.3 Comparing CPT Results of Sudbury Aggregates to Minimum SCM Requirements ... 118

5.4 Comparing CPT Results of Sudbury Aggregates to Spratt Aggregates....................... 120

5.5 Analyzing the Effects of Cement Content, Silane-sealer, and Sample Geometry ....... 123

5.6 Analyzing the Expansion in Extracted Cores and Road Barriers................................. 125

5.7 Analyzing the Expansion between RCA-M, RCA-H, and RCA-L.............................. 126

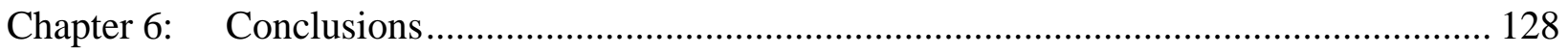


Chapter 7: Recommendations for Future work

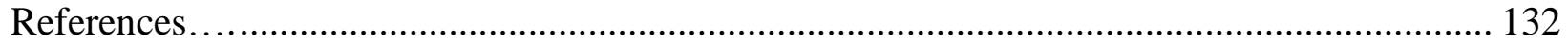




\section{List of Tables}

Table 2.1.1: Comparison of results between the CPT and AMBT

Table 3.2.1: Aggregate Properties

Table 3.2.2: Chemical Analysis of Cementing Materials

Table 4.1.1: Summary of 14-day expansion data for AMBT

Table 4.1.2: Summary of 28-day expansion data for CMBT

Table 4.1.3: Summary of 1-year and expected 2-year expansion data for CPT

Table 5.1.1: Comparing 1-year and expected 2-year expansion data for CPT of Sudbury and Spratt aggregates

Table 5.3.1: Minimum level of SCM required for Sudbury aggregates 


\section{List of Figures}

Figure 2.1.1: Process of Alkali-Silica Reaction

Figure 2.1.2: Pore Solution Concentration of Cement in First 24 Hours

Figure 2.1.3: Prism test expansion at 1 year given various cement contents

Figure 2.1.4: Comparison between crystalline structures of opal and quartz

Figure 2.1.5: Microscopic view of expansive ASR gel filling into a crack in Spratt RCA

Figure 2.1.6: Map cracking on bridge in Sudbury, Ontario

Figure 2.1.7: Horizontal cracking on road barrier

Figure 2.1.8: Localized cracking on bridge panel

Figure 2.1.9: ASR induced Pop-out

Figure 2.1.10: Concrete prism samples in storage bucket

Figure 2.1.11: 2-year CPT vs. 14-day AMBT expansion

Figure 2.1.12: Microbars in $1 \mathrm{~N} \mathrm{NaOH}$ solution at day 7

Figure 2.2.1: Microscopic view of air voids through use of air entrainment

Figure 2.2.2: Non-air entrained vs. Air-entrained

Figure 2.2.3: Use of Air Entrainment

Figure 2.2.4: Map cracking caused from freezing and thawing

Figure 2.2.5: D-Cracking at the surface joint

Figure 2.2.6: Scaling of concrete due to freeze-thaw deterioration

Figure 2.2.7: Pop-out due to frost susceptible aggregate

Figure 2.2.8: Number of freeze-thaw cycles vs. compressive strength

Figure 2.2.9: Air content vs. scaling due to freeze-thaw cycles

Figure 2.4.1: Deterioration of highway road barriers in Sudbury, Ontario

Figure 2.4.2: Low and high deteriorating road barriers from bridge condition survey conducted by MTO in 2011

Figure 3.1.1: Core drilling and measuring stud placement apparatus

Figure 3.1.2: Application of silane-sealer on cores

Figure 3.1.3: Cylinder mould for expansion testing

Figure 3.1.4: Graded RCA used for AMBT starting from retained on 2.36mm (left) to retained on $0.149 \mathrm{~mm}$ (right)

Figure 3.2.1: Gel formed from ASR exposed around aggregates at the surface of a concrete core taken from a bridge panel in Sudbury

Figure 4.1.1: AMBT Expansion results of Sudbury control and Sudbury containing fly ash

Figure 4.1.2: AMBT Expansion results of Sudbury control and Sudbury containing slag

Figure 4.1.3: AMBT Expansion results of Sudbury containing HSF and various SCM

Figure 4.1.4: AMBT summary of 14 day expansion results for virgin Sudbury aggregate

Figure 4.1.5: AMBT Expansion results of Sudbury RCA control and Sudbury RCA containing

fly ash

Figure 4.1.6: AMBT Expansion results of Sudbury RCA control and Sudbury RCA containing

slag

Figure 4.1.7: AMBT Expansion results of Sudbury RCA containing HSF and various SCM

Figure 4.1.8: AMBT summary of 14 day expansion results for Sudbury RCA-M

Figure 4.1.9: AMBT Summary of 14-day expansion results

Figure 4.1.10: AMBT Summary of 28-day expansion results

Figure 4.1.11: 28-day repeat results of AMBT

Figure 4.1.12: Spratt, Sudbury, and Sudbury RCA-M CMBT expansion results 
Figure 4.1.13: CMBT Expansion results of Sudbury control and Sudbury containing fly ash Figure 4.1.14: CMBT Expansion results of Sudbury control and Sudbury containing slag Figure 4.1.15: CMBT Expansion results of Sudbury containing HSF and various SCM Figure 4.1.16: CMBT summary of 28-day expansion results for virgin Sudbury aggregate Figure 4.1.17: CMBT Expansion results of Sudbury RCA control and Sudbury RCA containing fly ash

Figure 4.1.18: CMBT Expansion results of Sudbury RCA control and Sudbury RCA containing slag

Figure 4.1.19: CMBT Expansion results of Sudbury RCA-M containing HSF and various SCM Figure 4.1.20: CMBT summary of 28-day expansion results for Sudbury RCA-M

Figure 4.1.21: CMBT Summary of 28-day expansion results Figure 4.1.22: CMBT Summary of 56-day expansion results Figure 4.1.23: CPT Expansion results of Sudbury control and Sudbury containing fly ash Figure 4.1.24: CPT Expansion results of Sudbury control and Sudbury containing slag Figure 4.1.25: CPT Expansion results of Sudbury containing HSF and various SCM Figure 4.1.26: CPT summary of 65-week (1 year for Control) expansion results for virgin Sudbury aggregate

Figure 4.1.27: CPT Expansion results of Sudbury RCA control and Sudbury RCA containing fly ash

Figure 4.1.28: CPT Expansion results of Sudbury RCA control and Sudbury RCA containing slag

Figure 4.1.29: CPT Expansion results of Sudbury RCA-M containing HSF and various SCM Figure 4.1.30: CPT summary of 65-week (1 year for Control) expansion results for Sudbury RCA-M

Figure 4.1.31: CPT Summary of 65 week (1 year for control mixes) expansion results Figure 4.1.32: 1-year expansion results for control mixes containing GU Portland cement and expected 2-year expansion results for mixes containing SCM

Figure 4.1.33: 14-day AMBT vs. 1-year and expected 2-year CPT expansion for virgin Sudbury aggregate

Figure 4.1.34: 14-day AMBT vs. 1-year and expected 2-year CPT expansion for Sudbury RCA$\mathrm{M}$

\section{RCA-M}

Figure 4.1.35: 28-day CMBT vs. 1-year and expected 2-year CPT expansion for virgin Sudbury aggregate

Figure 4.1.36: 28-day CMBT vs. 1-year and expected 2-year CPT expansion for Sudbury RCAM

Figure 4.2.1: Prisms cast with virgin Sudbury aggregate with varying cement contents Figure 4.2.2: Prisms cast with RCA-H with varying cement contents Figure 4.2.3: Prisms cast with RCA-L with varying cement contents Figure 4.2.4: 52-week expansion of prisms cast with virgin Sudbury aggregate, RCA-H, and RCA-L

Figure 4.2.5: Cylinders cast with virgin Sudbury aggregate with varying cement contents Figure 4.2.6: Cylinders cast with RCA-H with varying cement contents Figure 4.2.7: Cylinders cast with RCA-L with varying cement contents Figure 4.2.8: 52-week expansion of cylinders cast with virgin Sudbury aggregate, RCA-H, and RCA-L 
Figure 4.2.9: High and low deteriorated cores

Figure 4.2.10: 52-week expansion of prisms and cylinders cast with standard mix

Figure 4.2.11: 52-week expansion reduction due to silane-based sealer on standard mix samples Figure 4.2.12: 52-week expansion of prisms and cylinders cast with bridge mix

Figure 4.2.13: 52-week expansion reduction due to silane-based sealer on bridge mix samples

Figure 4.2.14: 52-week expansion of high and low deteriorated cylinders and cores

Figure 4.2.15: 80 week expansion data for high and low deteriorated road barriers

Figure 4.2.16: 1-year expansion data of high and low deteriorated cores and road barriers

Figure 4.2.17: Comparing 1-year expansion results of RCA-M, RCA-H, and RCA-L

Figure 4.2.18: Comparing the effects of SCM and the silane-based sealer as an ASR mitigating technique

Figure 5.1.1: Effects of Portland cement alkali content on expansion of concrete prisms 


\section{Chapter 1: Introduction}

Years ago, concrete structures were built without worrying about the type of aggregate that would be used in the concrete mix. Starting in the mid- $19^{\text {th }}$ century, it has become apparent that it is important to know many characteristics about the mixture, such as the type of aggregate, before it is used to build structures. With any concrete structure, over time, deterioration will begin and the structure will continue to deteriorate due to a variety of mechanisms. In most cases, the structure can be monitored and repaired, or it can be neglected and left to deteriorate until it has reached a point that is deemed unsafe, in which the structure reaches the end of its service life. However, some deterioration modes in concrete cannot be properly mitigated or repaired after the structure is complete, such as Alkali-Silica reaction (ASR). Not only is it difficult to control the effects of ASR, but the deterioration mode is slow-acting, thus does not become apparent until many years after construction. If more information is known about the aggregate before being used, such as its susceptibility to ASR deterioration, preventative measures can be made in order to reduce or remove the deterioration that were to occur in the structure.

As structures reach the end of their service life, they need to be demolished and replaced. Demolished concrete structures produce a large amount of waste that sit in our landfills, consuming space while having large negative effects on our economy and environment. Recently, research is continuing to pick up on the use of demolished concrete structures as a building material in new concrete. As our awareness for the environment becomes more important in the construction industry with each year, it is important to strive to find new ways of being more environmentally friendly. One of these ways is by crushing demolished structures into coarse aggregate (generally $19.5 \mathrm{~mm}$ to $4.75 \mathrm{~mm}$ ), and using it as a recycled concrete aggregate (RCA). However, using RCA 
as a building material has some side effects that need to be evaluated before it can be used. First and foremost, it is important to know what aggregate was used in the original structure and what type of deterioration has occurred to that structure or aggregate. It is also important to note that the use of RCA might produce concrete of lower durability than concrete made with virgin aggregate. In addition, the absorption of RCA is much higher than that of virgin aggregate due to the presence of the old cement. Because if the reduction in durability, it is important to be extra cautious when building with RCA, especially in areas of harsh weather climates. These side effects create hurdles for the builder, thus the use of RCA is very minimal at this time, especially in Canada.

When dealing with RCA, extensive information on the original aggregate and the previous structure is crucial. Information on the aggregate, such as its durability and its susceptibility to various deterioration mechanisms are important to know in general, but more so when building with an RCA. Generally, the necessary information is readily available about types of virgin aggregate so the required precautions can be taken when using it, such as their durability or susceptibility. However, this information cannot be relied on if the plan is to use an RCA containing the same virgin aggregate due to the deterioration it has encountered in its original structure. For instance, if a structure suffered from freeze-thaw deterioration and ASR, it is important to test both the virgin aggregate and the RCA obtained from the structure in order to determine its usability. This includes determining if the original aggregate is frost susceptible and how reactive it is to ASR. It is also important to test the RCA obtained from the structure in order to determine the difference in durability, absorption, reactivity, and whether or not the deterioration that occurred in the previous structure will continue to deteriorate or aid in deteriorating the new structure. Thus, with continuing research, the comfort and use of RCA can 
become more popular in society, whether it is a durable aggregate or an aggregate that may be susceptible to freeze-thaw deterioration or ASR.

This thesis will examine the use of RCA produced from structures that suffered both ASR ad freezing/thawing damage as a building material. To do so, a variety of lab and field tests have been completed in order to satisfy a number of objectives. The RCA for this research came from two sources: high deteriorated road barriers and low deteriorated road barriers. The objectives of this research include: (1) to compare the expansion between virgin aggregate and RCA containing the same virgin aggregate, (2) to determine if RCA produced from high deteriorated road barriers expand more or less than that of RCA obtained from low deteriorated road barriers, (3) to determine if the accelerated mortar bar test (AMBT) and the concrete microbar test (CMBT) will predict the expansion obtained by the concrete prism test (CPT) for both moderately reactive virgin aggregate and RCA, and (4) to correlate lab and field data through the use of prisms, cylinder, cores, and road barriers. By comparing the level of expansion between the virgin aggregate and an RCA containing the same aggregate, the type and level of mitigation required for RCA can be determined. The next step includes determining whether or not the level of deterioration that has occurred in the previous structure effects the deterioration that will occur in the new structure. If not, the same mitigation techniques can be applied to both RCA produced from high deteriorated road barriers and low deteriorated road barriers. Then, tests were completed using the accelerated mortar bar test, the concrete microbar test, and the concrete prism test in order to confirm the validity of their ability to predict the expansion of concrete prisms. A variety of supplementary cementing materials (SCM) were used in this process in an effort to reduce the expansion of the RCA to a usable limit. Finally, by comparing expansion between the lab, with the use of concrete 
prisms, cylinders, and cores, an effort was made to correlate the lab data to data obtained using concrete road barriers in the field. 


\section{Chapter 2: Literature Review}

\subsection{Alkali-Silica Reaction}

Alkali-silica reaction (ASR) is a slow acting deterioration in concrete that only occurs when alkalis, reactive siliceous aggregate, and water are all present. As shown in Figure 2.1.1-A, the reaction occurs due to the mixture of sodium $\left(\mathrm{Na}^{+}\right)$and potassium $\left(\mathrm{K}^{+}\right)$ions with hydroxyl ions $\left(\mathrm{OH}^{-}\right)$, resulting in an increase of $\mathrm{pH}$ (Thomas, Fournier, and Folliard, 2013). Due to the high $\mathrm{pH}$ level, the hydroxyl ions intrude on the reactive silica $\left(\mathrm{SiO}_{2}\right)$, causing it to dissipate (Thomas, Fournier, and Folliard, 2013). When calcium is introduced, a gel is formed around the aggregate and the absorption of water from the surrounding cement paste causes expansion, shown in Figure 2.1.2-B and 2.1.2-C. This expansion results in a direct increase of pressure, unavoidably causing the concrete to crack (Thomas, Fournier, and Folliard, 2013).

A)

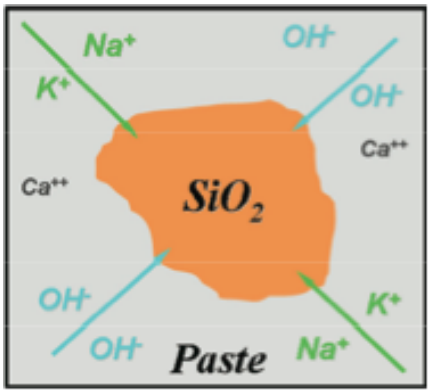

Ion reaction causing increased $\mathrm{pH}$
B)

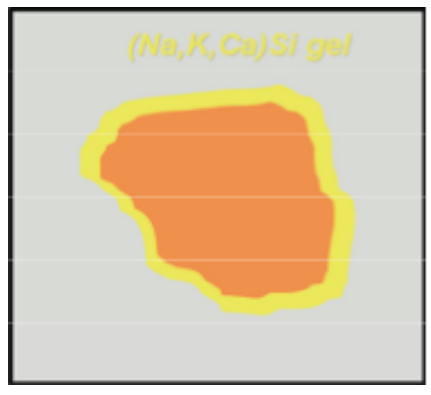

Calcium gel formation
C)

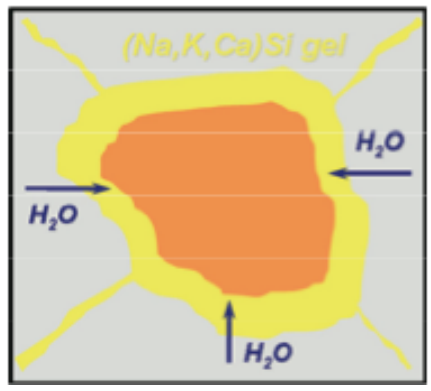

$\mathrm{H}^{2} 0$ causing gel expansion

Figure 2.1.1: Process of Alkali-Silica Reaction (Thomas, Fournier, and Folliard, 2013)

\subsubsection{Requirements for ASR}

\subsubsection{Alkalis}

The alkalis are mainly contributed from the Portland cement, which contains sodium and potassium, but can also be obtained from supplementary cementing materials (SCMs), aggregates, chemical admixtures, or external sources (Thomas, Fournier, and Folliard, 2013). Although 
cement paste generally contains less than $1 \%$ of sodium oxide equivalent $\left(\mathrm{Na}_{2} \mathrm{Oe}\right)$, which represents the alkali content by percent in the cement, the alkalis are highly soluble (Thomas, Fournier, and Folliard, 2013). Because of the solubility, they make up the majority of the pore solution of the cement. Shown below in Figure 2.1.2, within 24 hours, the concentration of the pore solution in cement paste becomes mainly potassium, hydroxyl, and sodium ions. Thus, the alkalis are able to move easily within the paste and attack the aggregate.

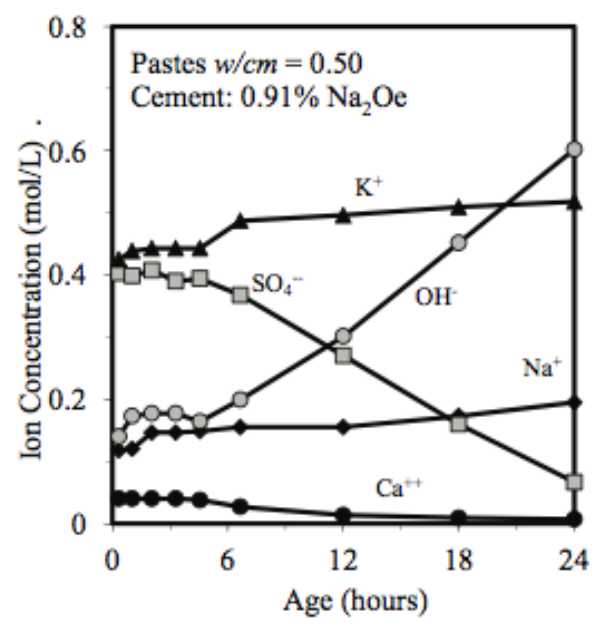

Figure 2.1.2: Pore Solution Concentration of Cement in First 24 Hours (Diamond, 1983)

It was earlier proposed that using cement with limited alkali content would reduce the risk of alkali-silica reaction (Stanton, 1940). However, a new approach of limiting the total alkali content on the concrete has since been preferred (Thomas, Fournier, and Folliard, 2013). This may be done by decreasing the amount of cement in the mix design, such as $275 \mathrm{~kg} / \mathrm{m}^{3}$ instead of $420 \mathrm{~kg} / \mathrm{m}^{3}$. Figure 2.1.3 shows expansion data at 1 year for the concrete prism test of specimens with siliceous limestone at various cement contents and total concrete alkali contents. It is shown that having a total concrete alkali content below $3 \mathrm{~kg} / \mathrm{m}^{3}$ is favourable. This can be obtained in a combined effort of low alkali cement and lower cement content within the concrete. 


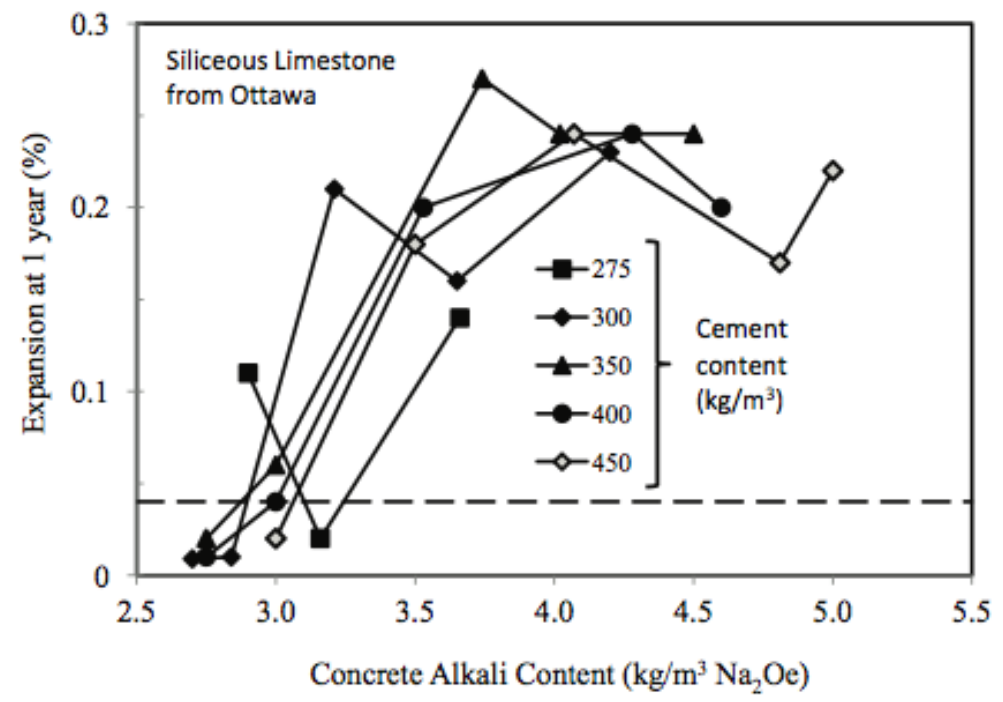

Figure 2.1.3: Prism test expansion at 1 year given various cement contents (Thomas, Fournier, and Folliard, 2013)

\subsubsection{Aggregate Type}

The type of aggregate used in the concrete is important to note as many stones contain silica $\left(\mathrm{SiO}_{2}\right)$. With that taken into consideration, only aggregates that contain reactive silica will cause ASR to occur. Some sources of reactive siliceous aggregates include opal, chalcedony, rhyolite, and volcanic glass (Thomas, Fournier, and Folliard, 2013). Aggregates that contain disordered structures are unstable at high $\mathrm{pH}$ levels thus are far more susceptible to reacting with the alkalis, causing expansion (Thomas, Fournier, and Folliard, 2013). Figure 2.1.4 shows a comparison between two crystalline structures, opal (highly susceptible to ASR) and quartz (not susceptible to ASR). It is clear that opal allows for the alkalis to easily enter and access the silica due to its disoriented structure. On the contrary, quartz has a very well structured system that guards the silica from the alkalis, thus not allowing the reaction to occur. 


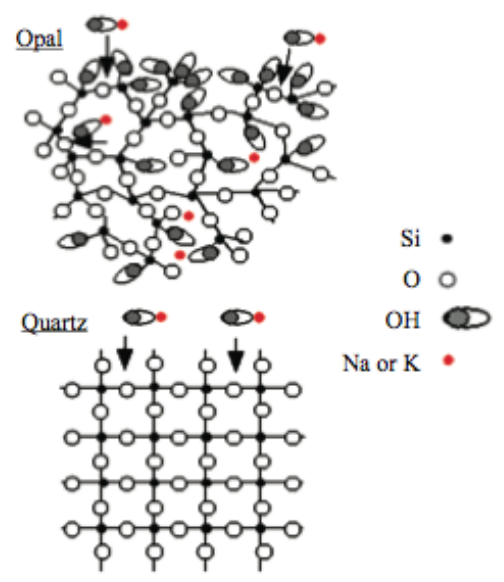

\section{Figure 2.1.4: Comparison between crystalline structures of opal and quartz (Thomas, Fournier, and Folliard, 2013)}

\subsubsection{Moisture}

In order for the reaction to occur, it is said that the internal relative humidity within the concrete should be at least $80 \%$. Differential moisture within a structure can cause different levels of ASR within the same structure (Thomas, Fournier, and Folliard, 2013). Essentially, if a structure is an area where a portion is in constant moisture and another portion is constantly dry, the moist portion can suffer from ASR and expand while the dry section will not. Thus, if the structure can be kept away from moisture and constantly dry, ASR cannot occur.

\subsubsection{Alkali-Silica Reaction Indicators}

When concrete begins to deteriorate, it is essential to determine the cause of the deterioration. There are several visual indicators that may be analyzed in order to determine the source of the deterioration. Some indicators of ASR include the type of cracking, deformation due to expansion, localized deterioration of concrete, presence of expansive gel, and pop-outs (Thomas, Fournier, and Folliard, 2013). It is crucial to be aware of the characteristics when diagnosing the deterioration mechanism in order to control and limit the issue. 
Samples of the concrete can also be polished and looked at under a microscope to determine what is causing the expansion. In order to confirm ASR caused expansion or deterioration to concrete, it is important to do so. Figure 2.1.5 shows a crack in a recycled concrete aggregate filled with ASR expansive gel.

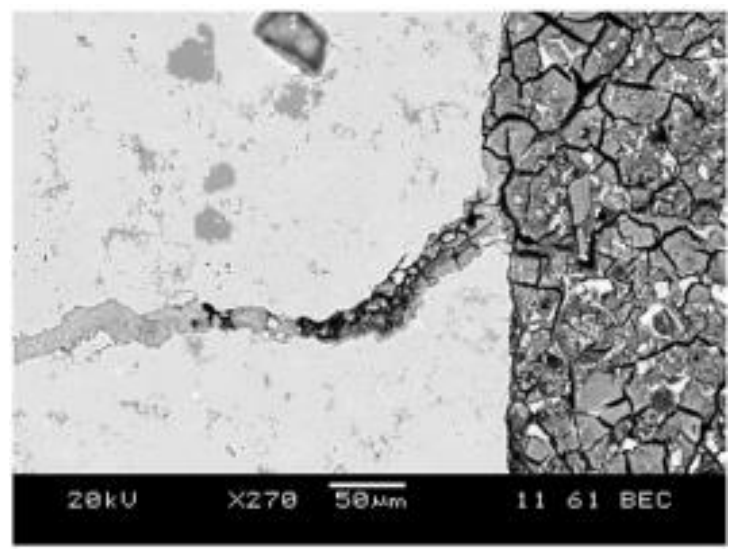

Figure 2.1.5: Microscopic view of expansive ASR gel filling into a crack in Spratt RCA (Shehata et al, 2010)

\subsubsection{Cracking}

Cracking caused by alkali-silica reaction is normally portrayed at the surface as 'map' cracking, similar to that of freezing and thawing. Map cracking is a cause of little or no restraint in all directions causing random cracking on the surface (Thomas, Fournier, and Folliard, 2013). Figures 2.1.6-A and 2.1.6-B show two cases of map cracking from a bridge in Sudbury, Ontario. 
A)

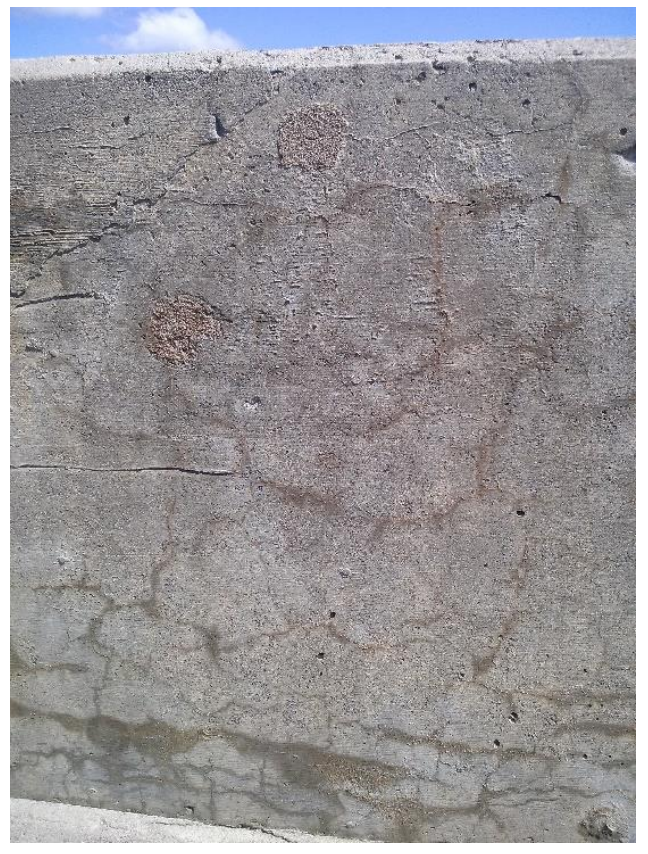

Map cracking on road barrier
B)

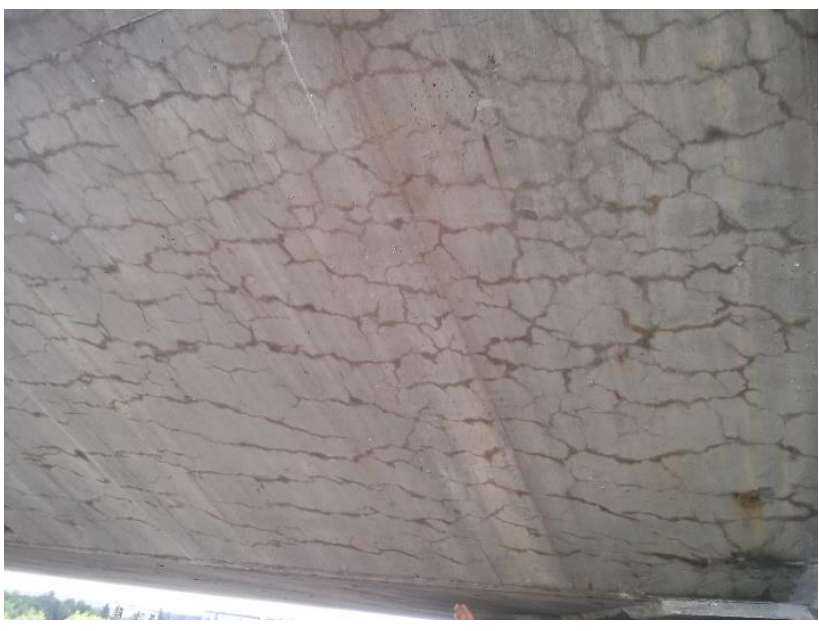

Map cracking on underpass of highway

Figure 2.1.6: Map cracking on bridge in Sudbury, Ontario

Figure 2.1.6-A depicts map cracking on the backside of a highway wall barrier. It is evident that there is some localization of ASR as the cracks appear much more concentrated near the bottom of the wall barrier. Figure 2.1.6-B displays a large area of map cracking on the bottom side of a bridge, which appears as though there is no localization of cracking. There is also a noticeable change in colour where the cracks are present, which are often caused by the gel migrating through the cracks (Thomas, Fournier, and Folliard, 2013).

Certain factors can alter the formation of cracks such as the shape and size of the concrete, environmental conditions, types of loads applied to the concrete member, and restrains from reinforcement within the concrete. For example, if horizontal reinforcement is present in the concrete, it can cause the cracks to run parallel of the rebar. In Figure 2.1.7, a wall barrier shows horizontal cracking along the top end of the barrier. It is apparent that the horizontal cracking is 
caused by the restriction applied by the rebar running parallel, as shown on the cross-section of the barrier.

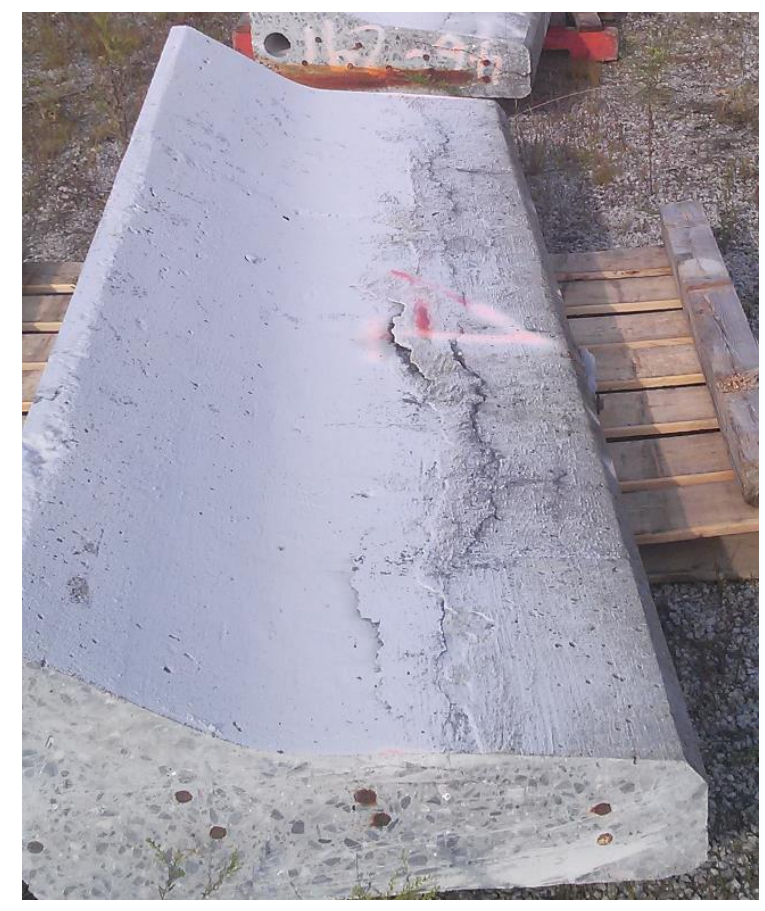

Figure 2.1.7: Horizontal cracking on road barrier

Figure 2.1.7 shows that although the rebar may restrict the cracking from moving into the perpendicular direction, it does not reduce the effect of the deterioration on the surface. Adversely, the cracking becomes larger and more imminent in the horizontal direction.

Localized cracking can occur under a few circumstances. If only a portion of the concrete is experiencing ASR or a portion of the concrete is more susceptible to ASR given the environmental conditions, that portion will expand quicker and will crack at an earlier time than the rest of the structure. For example, if a portion of the concrete is located indoors without moisture present, and the rest of the structure is constantly in contact with moisture, it is likely that localized cracking will occur on the moist portion of the concrete. Similarly, if one portion is under constant moisture while another portion is under periodic moisture, the portion that is constantly 
moist will experience quicker expansion, thus resulting in more imminent and apparent cracking. In Figure 2.1.8, localized cracking is shown on a bridge panel from Sudbury, Ontario as there is aggressive and concentrated cracking near the top of the panel. The cracking begins to become less apparent and almost non-existent as it approaches the bottom of the panel.

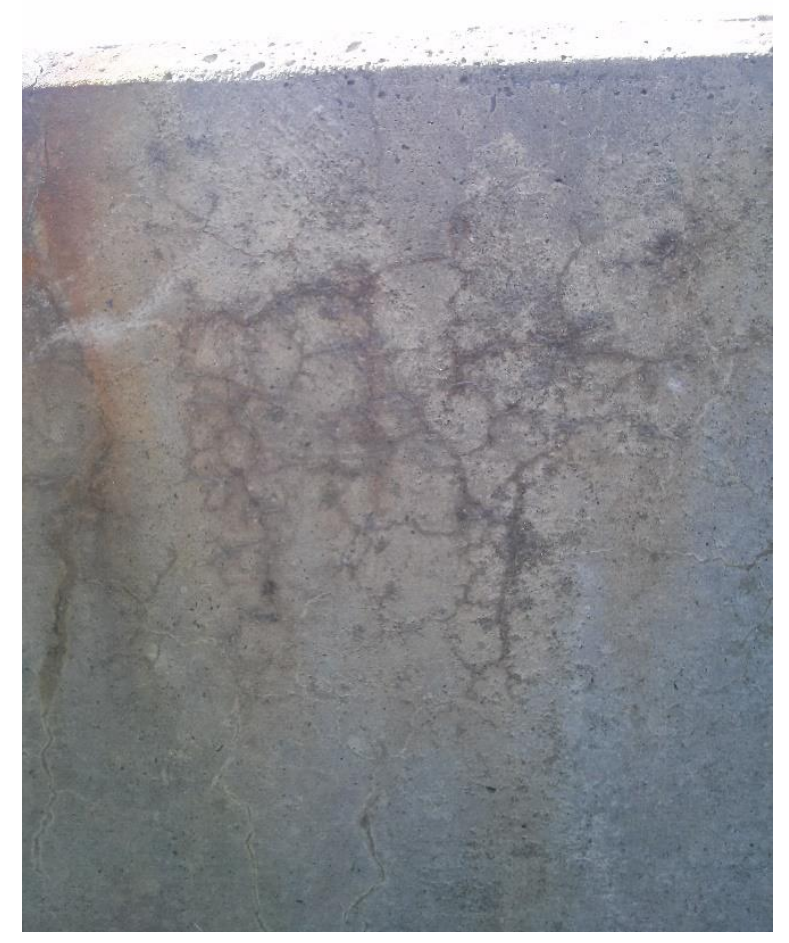

Figure 2.1.8: Localized cracking on bridge panel

\subsubsection{Pop-outs and Gel Presence}

Pop-outs are not as common in ASR as they are a result of other deterioration mechanisms such as frost action or poor cement and aggregate bond. However, they can occur if the reaction occurs close to the surface (Thomas, Fournier, and Folliard, 2013). This is due to the expansion of the aggregates undergoing ASR near the surface. Since the stress required to cause a pop-out near the surface is far less than the stress required to cause cracking within the concrete, the cracking tends 
to occur near the surface. A pop-out caused by ASR can be identified by the gel that begins to expose at the surface of the concrete. Figure 2.1.9 shows a pop-out with gel surrounding the crack.

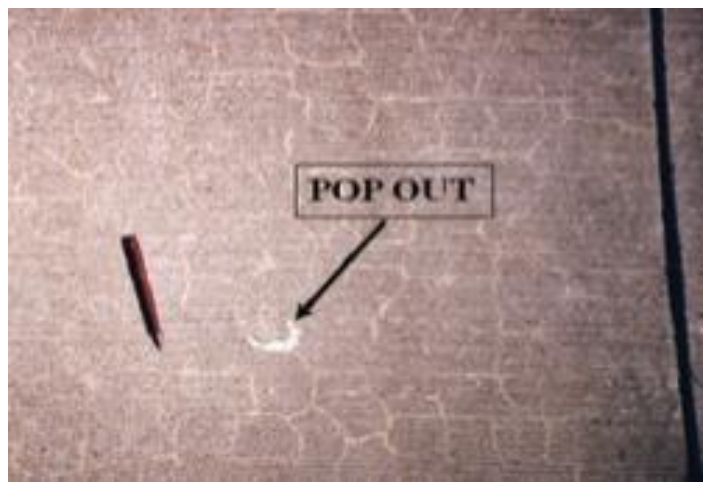

Figure 2.1.9: ASR induced Pop-out (Thomas, Fournier, and Folliard, 2013)

\subsubsection{Alkali-Silica Reaction Lab Testing Methods}

There are several test methods than can be carried out in the lab in order to determine the reactivity of the aggregate prior to its use. These test methods include the concrete prism test (CPT), accelerated mortar bar test (AMBT), and concrete microbar test (CMBT). These test methods aims at accelerating the rate of reaction to obtain results in a relatively short period of time. The amount of expansion for a given aggregate is determined in order to understand the susceptibility of the aggregate and determine whether or not it is safe to use. Preventative measures, such as the application of sealers and the use of supplementary cementing materials (SCMs) can be used in order to reduce the expansion. Once the desired expansion results are obtained, an understanding of the type of aggregate and the level of preventative measures required is obtained.

\subsubsection{Concrete Prism Test}

The concrete prism test is the most reliable ASR lab testing method. It consists of casting three prisms with alkalis raised to $1.25 \% \mathrm{Na}_{2} \mathrm{O}_{e}$ and a cement content of $420 \mathrm{~kg} / \mathrm{m}^{3}$. The prisms are then positioned above water in a container and placed in $100 \%$ humidity at $38^{\circ} \mathrm{C}$. The downfall is that 
the test requires 1 year to obtain completed results for concrete containing virgin aggregate and 2 years for concrete containing SCMs, where the expansion limit is 0.04\% (CSA A23-25A, 2009). Figure 2.1.10 shows a set of 3 concrete prisms samples placed in a bucket elevated over water. Once the lid is sealed, the samples are ready to be placed into the heat room set at $100 \%$ humidity and $38^{\circ} \mathrm{C}$. This procedure is designed to obtain accelerated results, with readings taken at specific times throughout the year(s), to determine the reactivity of the aggregate.

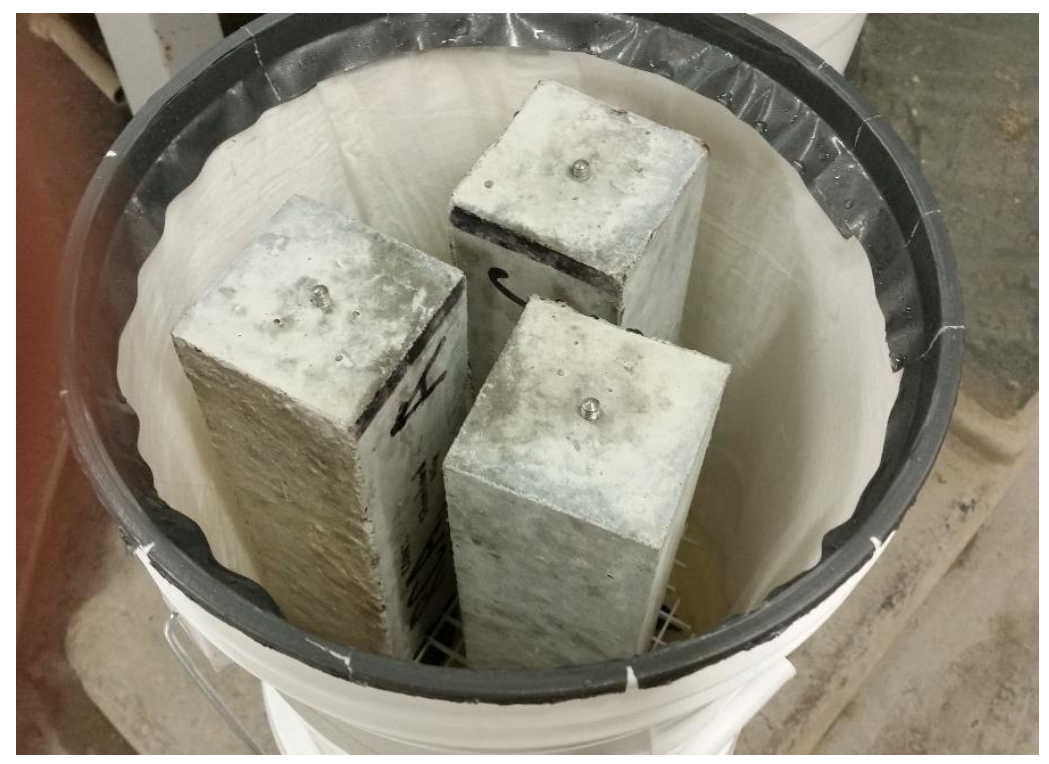

Figure 2.1.10: Concrete prism samples in storage bucket

\subsubsection{Accelerated Mortar Bar Test}

The accelerated mortar bar test consists of creating three samples that contain crushed forms of the aggregate and graded in accordance to CSA A23.2-25A (2009). The samples are soaked in $1 \mathrm{~N}$ of sodium hydroxide $(\mathrm{NaOH})$ solution and the test has a total duration of 28 days. At 14 days, CSA A23.2-25A (2009) indicates that expansion results greater than $0.15 \%$ are considered reactive ( $0.10 \%$ for limestone). Although results can be obtained in less than a month, these results should not be analyzed independently. 
It is important to correlate the data obtained from the AMBT with data from the concrete prism test. The following chart (Figure 2.1.11) shows a comparison between 2-year data of the prism test and 14-day data of the AMBT to determine its reliability. The figure shows that the reliability of the results obtained from the mortar bar test becomes problematic when the data is very close to the expansion limit. For example, the one data point that lies directly on the $0.04 \%$ limit for the CPT is beyond the limit of $0.1 \%$ for the AMBT. Thus it is important to carry out the CPT test before declaring the aggregate non-reactive.

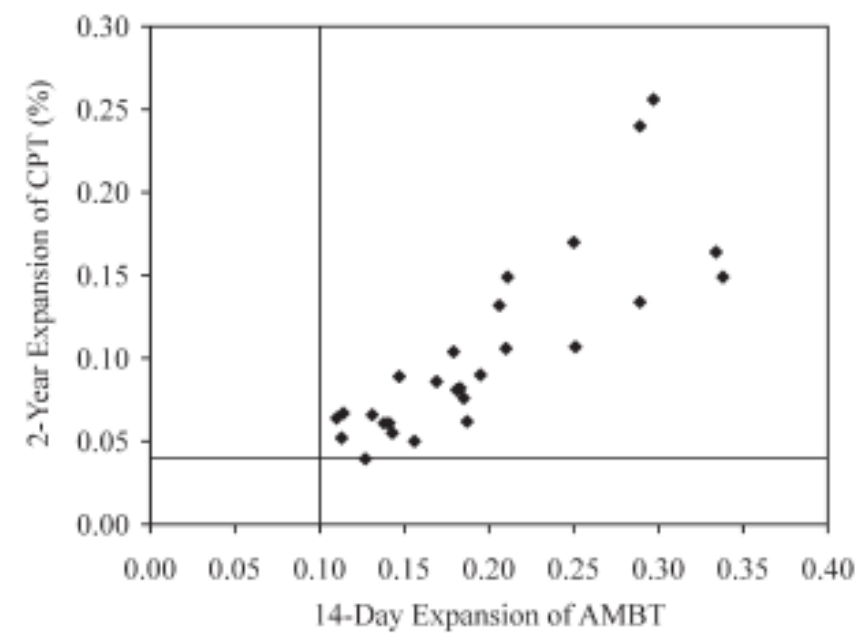

Figure 2.1.11: 2-year CPT vs. 14-day AMBT expansion (Shehata et al, 2010)

Table 2.1.1 below further explains that there are some discrepancies within the results obtained between the two tests. There are circumstances in which the concrete prisms test passes and the accelerated mortar bar test fails - of the 184 tests completed, there were $9(4.9 \%)$ occurrences. Similarly, there were 3 cases (1.6\%) in which the AMBT passed and the CPT failed. Although these numbers may be low and seem insignificant, there were an additional $73(40 \%)$ tests that were deemed inconclusive. The inconclusive data represents cases in which the expansion was too close to the limit to have confidence that it had passed or failed the test. These inconclusive zones were determined to be 1.96 times the standard deviation, thus the AMBT inconclusive zone was 
$0.10 \%+/-0.03 \%$ and the CPT inconclusive zone was $0.04 \%+/-0.018 \%$. Therefore, there was a combined 85 cases, or $46.5 \%$ of the time, in which the two tests were unable to confirm the same conclusion. Thus, it is recommended to wait for the results obtained from the CPT, as it is regarded to as the most reliable test method for ASR.

\begin{tabular}{|c|c|c|}
\hline $\begin{array}{l}\text { Concrete } \\
\text { prism test }\end{array}$ & $\begin{array}{l}\text { Accelerated } \\
\text { mortar bar test }\end{array}$ & $\begin{array}{l}\text { Frequency } \\
\text { of outcome }\end{array}$ \\
\hline Pass $(<0.012 \%$ at 2 years) & Pass ( $<0.070 \%$ at 14 days) & $33(18 \%)$ \\
\hline Fail $(>0.052 \%$ at 2 years) & Fail $(>0.130 \%$ at 14 days $)$ & $66(36 \%)$ \\
\hline Pass $(<0.012 \%$ at 2 years) & Fail $(>0.130 \%$ at 14 days $)$ & $9(4.9 \%)$ \\
\hline Fail ( $>0.052 \%$ at 2 years) & Pass ( $<0.070 \%$ at 14 days) & $3(1.6 \%)$ \\
\hline \multicolumn{2}{|c|}{$\begin{array}{l}\text { Inconclusive (CPT }=0.012 \% \text { to } 0.052 \% \\
\text { AMBT }=0.070 \% \text { to } 0.130 \% \text {, or both) }\end{array}$} & $73(40 \%)$ \\
\hline \multicolumn{2}{|l|}{ Total } & 184 \\
\hline
\end{tabular}

Table 2.1.1: Comparison of results between the CPT and AMBT (Thomas et al., 2006)

\subsubsection{Concrete Microbar Test}

The microbar test consists of creating three samples that contain aggregate within the $4.75 \mathrm{~mm}$

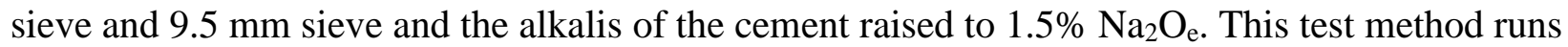
similarly to that of the accelerated mortar bar test. The samples must be soaked in $1 \mathrm{~N}$ of $\mathrm{NaOH}$ solution and run for 28 days. The results should not solely be relied on. Figure 2.1.12 shows a set of microbars that have been placed in the oven, under the same conditions as mortar bars. The samples are elevated to allow the solution to access all areas of the bars. A lid is placed on the container and the microbars are placed in an over set to $80^{\circ} \mathrm{C}$. 


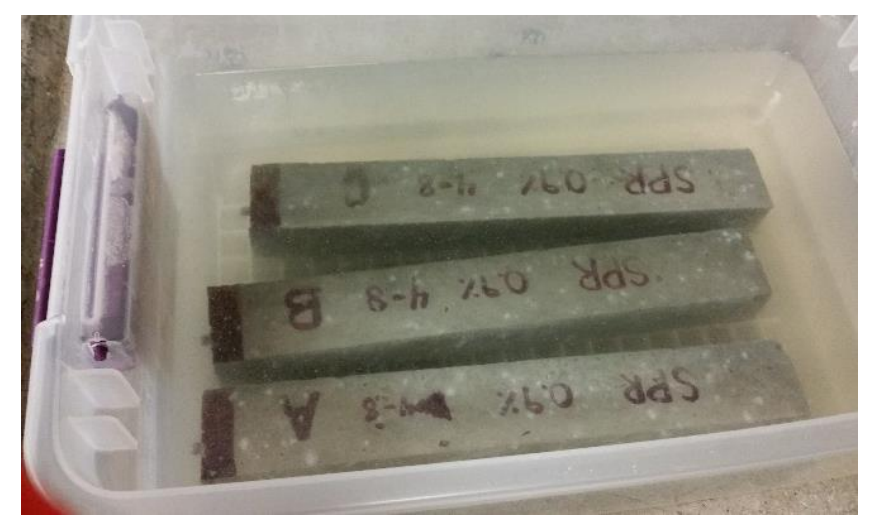

Figure 2.1.12: Microbars in $1 \mathrm{~N}$ NaOH solution at day 7

\subsection{Freeze-Thaw Deterioration}

Freezing and thawing is a cycle primarily dependent on weather conditions that causes concrete to deteriorate. The concept of freeze-thaw deterioration is as such: under lower temperature conditions, the water within concrete's pores will freeze, resulting in the expansion of concrete. It is estimated that water expands about 9\% when it freezes (Portland Cement Association, 2014). Over a series of numerous freeze-thaw cycles, the expansion inevitably results in stress within the concrete. In order to relieve the stresses, cracks in the concrete develop. Although the concept of freezing and thawing in concrete is simple, there are various factors constantly affecting it, causing excessive difficulty when determining the concrete's susceptibility. The best method to limit the concrete's susceptibility to freezing and thawing is to use local experimental data that takes the weather conditions into account and applies requirements for concrete design such as watercement ratio, air characteristics, and curing procedures (Ronning, 2001). There are a number of laboratory test results that may also be used to aid the determination of frost susceptibility such as determining the frost susceptibility of the aggregate and running freezing and thawing cycles on samples of different aggregates, water-cement ratios, and air entrainment in the lab. After running 
accelerated freezing and thawing cycles on these samples, the frost susceptibility of the concrete can be found by running them through cracking and scaling tests.

\subsubsection{Causes for Freeze-Thaw Deterioration}

There are a number of factors that will promote deterioration due to freezing and thawing in concrete. Using frost susceptible aggregates, lack of air entrainment, high water-cement ratio, and deicing salts are the main causes of freeze-thaw deterioration. Any of those factors in combination with saturated, improperly cured concrete in harsh weather climates, such as Canadian winters, will aid in the level of deterioration. Figure 2.2.1 shows a microscopic view of concrete that allows for roughly $10 \%$ expansion through freezing and thawing by creating air voids through the use of air entrainment (Sika, 2014). By using air entrainment to create air voids within the concrete, there is now more room for water to penetrate the concrete, making it less likely for the concrete to be fully saturated. When concrete is fully saturated, it is most susceptible to freeze-thaw deterioration.

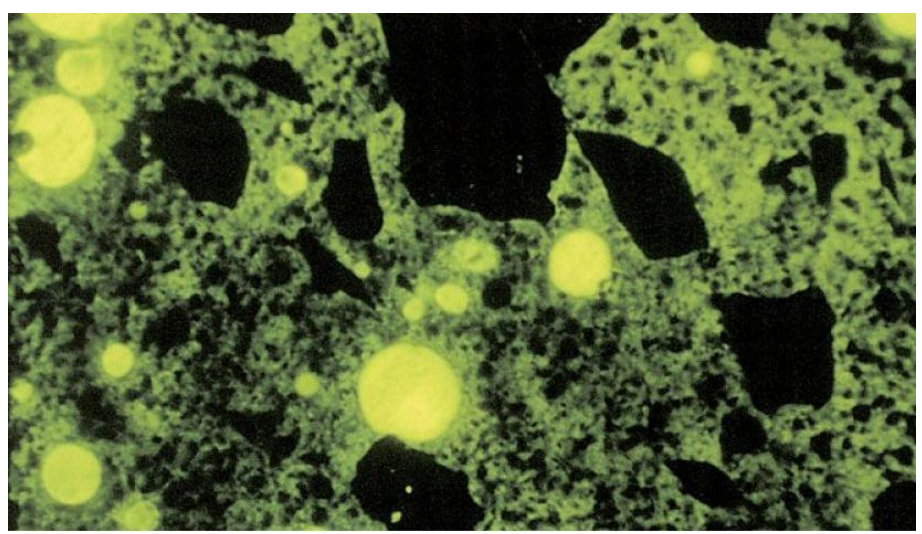

Figure 2.2.1: Microscopic view of air voids through use of air entrainment (Sika, 2014)

\subsubsection{Frost Susceptible Aggregates}

Some aggregates are more susceptible to freezing and thawing damage than others. This is largely due to the porosity of the aggregate; the more porous the aggregate, the more water it can absorb. It was concluded "it would be difficult to prove that any other physical property is of greater 
importance than the porosity characteristics in either natural or artificial aggregates" (Salcedo, 1984). The hydraulic pressure generated within the aggregate is the major cause of freezing and thawing damage. In addition, as permeability of the aggregate increases, internal hydraulic pressure decreases (Salcedo, 1984). Thus, it is the pore size that is of importance rather than the number of pores. Large amounts of small pores in an aggregate can lead to suctioning of water, resulting in fully saturated aggregate (Ronning, 2001). As the water freezes within the fully saturated aggregate it expands and cracks the aggregate, resulting in stress on the concrete. The end result is either a pop-out (if the aggregate is near the surface of the concrete) or internal cracking. Thus, it is important to ensure strong, durable aggregates are being used in areas where freezing and thawing deterioration is of concern.

\subsubsection{Air Entrainment}

The use of air entrainment admixtures allows you to intentionally create air bubbles within the concrete. These air bubbles are spaced evenly, about 0.01 inches apart and roughly the same order of magnitude in diameter (Hodgson, 2000). The main purpose of using air entrainment is to cope with freezing and thawing deterioration. The following figure shows the difference between two identical concrete samples, one without air entrainment (left) and the other with air entrainment (right). As shown in Figure 2.2.2 below, the tiny, pin-sized voids evenly spaced in the sample on the right are the air-entrained bubbles while the larger voids that appear in both samples are the entrapped air voids (Portland Cement Association, 2014). 


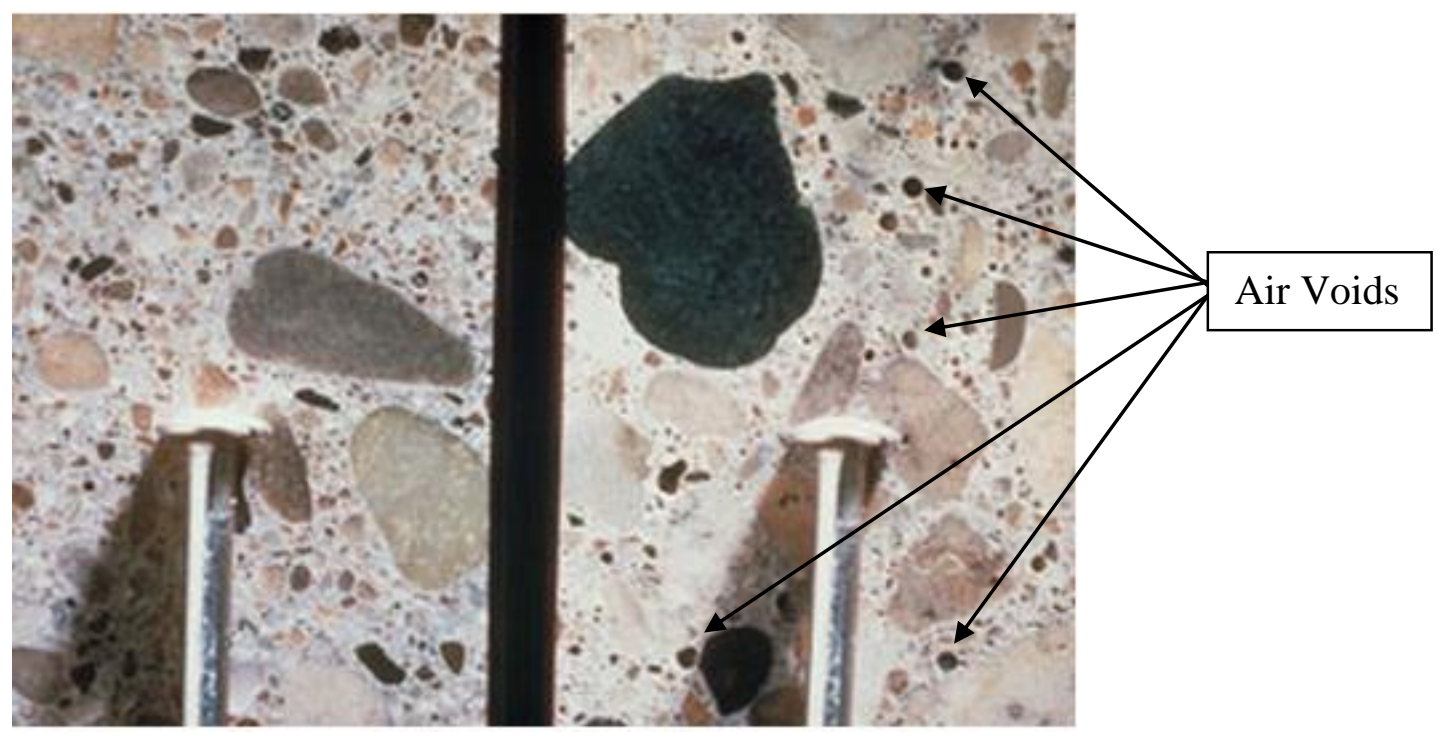

Figure 2.2.2: Non-air entrained vs. Air-entrained (Portland Cement Association, 2014)

In creating these small air bubbles, "the tiny entrained air voids act as empty chambers in the paste for the freezing and migrating water to enter, thus relieving the pressure in the pores and preventing damage to the concrete" (Portland Cement Association, 2014). Essentially, the air entrainment creates channels that connect neighboring pores within the concrete. As hydraulic pressure increases, these channels allow the freezing and expanding water to move to other pores rather than expanding and cracking the pore it currently inhabits. It is said that concrete exposed to freezing and thawing cycles should contain about $6 \%$ air content by volume (Mamlouk \& Zaniewski, 1999). Figure 2.2.3 shows the concept behind the migration of the water from pores to air bubbles created by the air entrainment. It allows for water built up in fully saturated pores to access other pores as the pressure builds. 


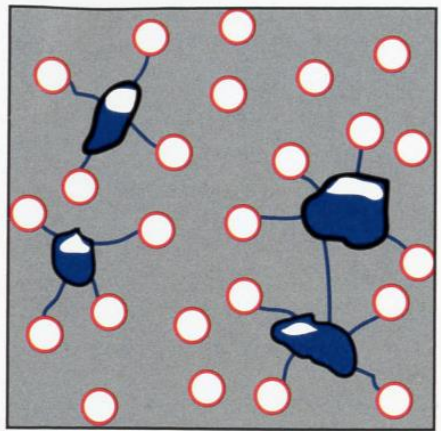

As temperatures drop, pores created by air entrainment allow the water a place to go as it freezes.

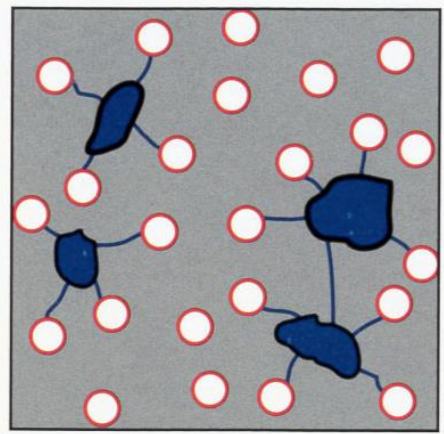

During freezing, water in the capillary pores expands; however, water is also going toward airexpands; however, pores.

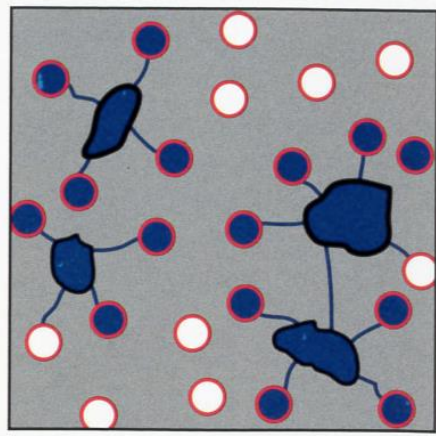

Under pressure, the water will be pushed into the ais entrainment pores and not crack the concrete matrix.

Figure 2.2.3: Use of Air Entrainment (Goguen, 2012)

\subsubsection{Water-Cement Ratio}

The water-cement ratio in a concrete mixture plays a vital role in the concrete's ability to resist freezing and thawing deterioration. If concrete is casted with a high water-cement ratio, the excess water in the mix will migrate to the top of the slab and eventually evaporate. In the process of migrating to the top, small channels known as capillaries are created and remain in the concrete when it has hardened (Hodgson, 2000). This creates a number of pores through the concrete resulting in a less dense and porous concrete. With this increased porosity, the strength of the concrete is reduced thus allowing more free water to penetrate the concrete (Hodgson, 2000).

\subsubsection{Deicing Salts}

When deicing chemicals such as ammonium sulfate or ammonium nitrate are applied onto saturated concrete, the water at the surface of the concrete begins to dissolve the chemicals and channels them throughout the concrete (National Ready Mixed Concrete Association, 1998). As the chemicals mix within the water, it attacks the concrete and begins to eat away at it, causing scaling to the surface of the concrete. 


\subsubsection{Indicators for Freeze-Thaw Deterioration}

There are several factors that contribute to concrete deterioration. Certain indicators allow us to determine the deterioration mechanism. When freezing and thawing deterioration occurs, it will likely result in surface scaling, pop-outs and cracking. The cracking will begin where the water is stored, begins to freeze, and will make their way to the surface. Cracking may be in the form of horizontal cracking, vertical cracking, map cracking, and D-cracking. The damage due to surface scaling significantly increases when deicing chemicals such as salt are used (Ronning, 2001).

\subsubsection{Internal Cracking}

Cracking due to freezing and thawing may begin as interior cracks, thus deterioration will not show immediately. The cracking can originate within the aggregate if it is a non-durable and porous aggregate, known as a frost susceptible aggregate. When dense, durable aggregates are used, it is likely that the deterioration will initiate in the cement paste (Ronning, 2001). In saturated conditions, water accumulates in the pores of the concrete. If the pores become fully saturated and temperatures begin to decrease, the water begins to freeze and expand and the pores will not be able to contain the volume expansion. If there are nearby connecting pores, the water will move to these areas. Once there is nowhere else for the water to move, the volume expansion will cause stress inside the concrete until stress is relieved, thus cracking occurs. It is common to see freezing and thawing damage result in map cracking, as shown in Figure 2.2.4. 


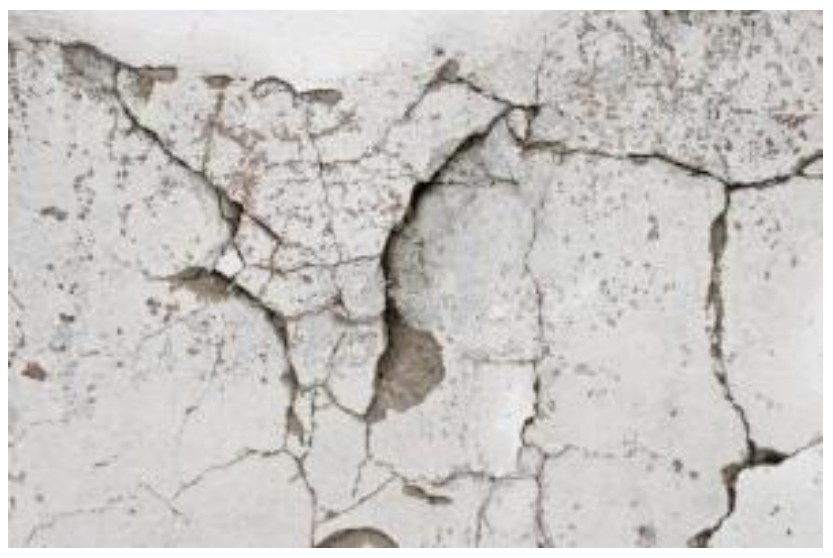

Figure 2.2.4: Map cracking caused from freezing and thawing (Seymour, 2014)

\subsubsection{D-Cracking}

D-Cracking is caused by accumulation of water under concrete pavement in the base and subbase, causing saturation to the aggregates. This is done through capillary action. As freezing and thawing cycles continue, cracking begins at the bottom and the cracks translate to the surface (Portland Cement Association, 2014). The cracks are normally very close to each other and appear near joints. They can form parallel or transversely and spread as they reach the surface. In Figure 2.2.5, D-cracking appears on the surface of the concrete near the joint. The cracking has spread in both directions however, the majority of the cracking has occurred parallel to the joint.

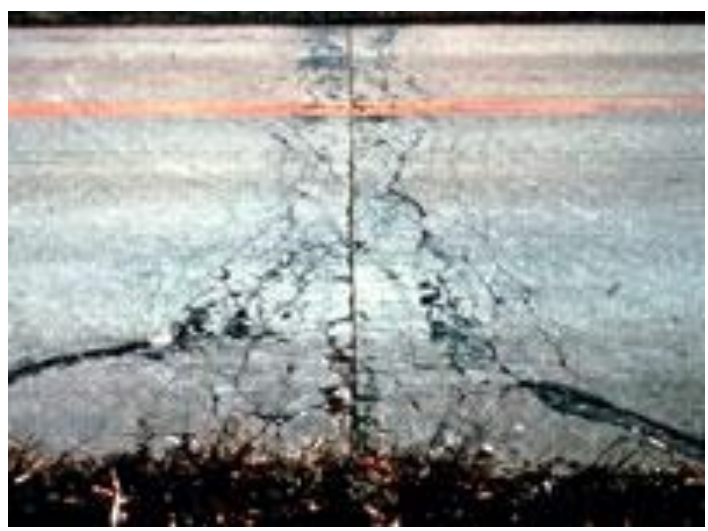

Figure 2.2.5: D-Cracking at the surface joint (Portland Cement Association, 2014) 


\subsubsection{Scaling}

Scaling of concrete may occur due to freezing and thawing deterioration in concrete. Scaling is caused by the combined action of freezing and thawing and the use of deicing chemicals (Portland Cement Association, 2014). Although the deterioration is slow acting, scaling can cause severe damage over time and expose reinforcement. Figure 2.2.6-A shows a mild case of scaling while figure 2.2.6-B shows the effects of scaling over a long period of time.

A)

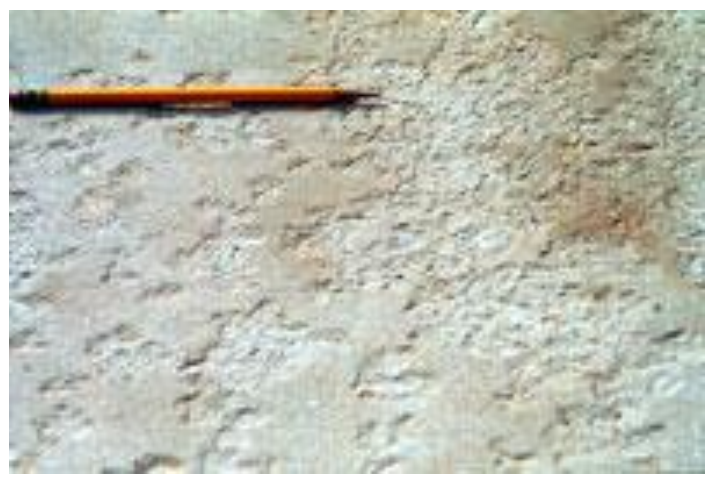

Scaling due to freeze-thaw deterioration
B)

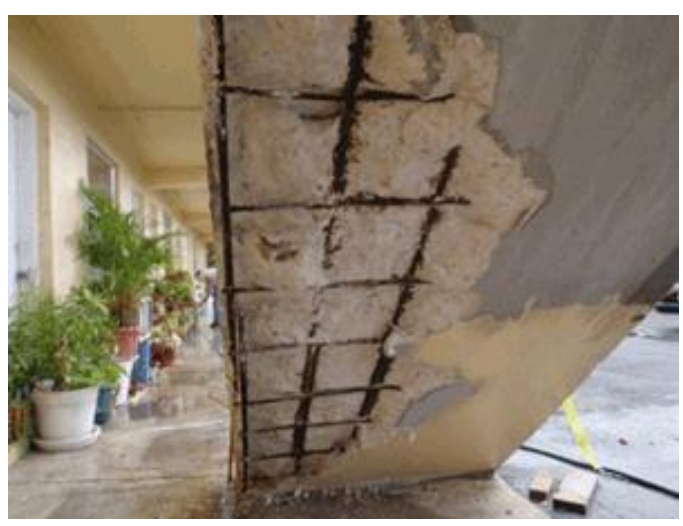

Exposed reinforcement due to scaling

Figure 2.2.6: Scaling of concrete due to freeze-thaw deterioration (Portland Cement Association, 2014)

\subsubsection{Pop-outs}

Pop-outs due to freezing and thawing can occur when a frost susceptible aggregate becomes fully saturated, or reaches saturated surface dry (SSD) conditions near the surface of the concrete. Porous aggregates are more frost susceptible as they have the ability to easily absorb water. When the aggregate is fully saturated and the temperature decreases, the water within the aggregate freezes. This expansion within the aggregate results in cracking. When this occurs near the surface, the pressure from the aggregate causes the paste above it to separate from the concrete and causes a pop-out. Figure 2.2.7 shows a pop-out caused by the use of frost susceptible aggregate. 


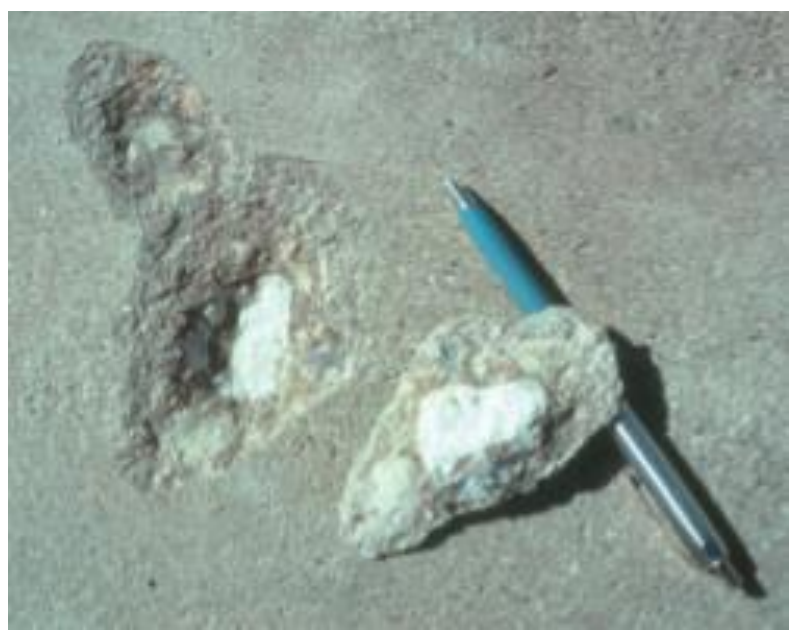

Figure 2.2.7: Pop-out due to frost susceptible aggregate (Thomas, Fournier, and Folliard, 2013)

\subsubsection{Freeze-Thaw Deterioration Lab Testing Methods}

There are several test methods that can be performed in the lab to reduce the risk of casting a frost susceptible concrete. This includes testing the aggregates being used or testing a full concrete mix. To test aggregates, the resistance of coarse aggregates to freezing and thawing test is used. In order to test concrete specimens there are a number of tests that can be completed that generally incorporate varying the amount of entrained air and water-cement ratios. These samples are then put through freezing and thawing cycles to compare results. Some of these test methods include testing the degree of saturation, internal cracking, and scaling resistance (Ronning, 2001).

\subsubsection{Coarse Aggregate Resistance}

This test method is used to determine the durability of an aggregate that will be exposed to freezing and thawing. The coarse aggregate is placed in containers filled with a $3 \%$ sodium chloride solution, which undergo five freezing and thawing cycles. The sample is then washed, dried, and weighed to determine the percentage loss by mass. The higher the percent loss, the more frost 
susceptible the aggregate is. In order for the aggregate to be deemed frost resistant, the percent loss shall be less than 6\% (CSA A23.1-09, 2009).

\subsubsection{Degree of Saturation}

The degree of saturation test is used to determine the maximum level of saturation that will not cause damage to the specimen. The test incorporates pre-wetting samples to different levels of saturation and running them through freezing and thawing cycles. After a number of freezing and thawing cycles are performed, various tests can be completed on the samples such as determining the dynamic modulus of elasticity. By comparing the results, the critical degree of saturation can be determined. Therefore, if the critical degree of saturation is higher than the actual degree of saturation, the concrete is considered to be frost resistant (Ronning, 2001).

\subsubsection{Internal Crack Test}

The beam test, found in ASTM C666 (2015) "Standard Test Method for Resistance of Concrete to Rapid Freezing and Thawing" consists of putting samples through two different cycles. The first cycle type being rapid freezing and thawing in water and the second being rapid freezing in air and thawing in water. The test procedure for both cycles consists of temperatures changing from $4{ }^{\circ} \mathrm{C}$ to $-18{ }^{\circ} \mathrm{C}$ with at least $25 \%$ of the time used for thawing (ASTM C666, 2015). The basis of the assessment is to test the beams for dynamic modulus of elasticity in order to determine the internal cracking that has occurred through the freezing and thawing cycles (Ronning, 2001). The following data shows the effects on compressive strength of concrete given a number of rapid freeze-thaw cycles and varying water cement ratios. 


\begin{tabular}{lcccccccc}
\hline & W/C & Cement & $\begin{array}{c}\text { Cement } \\
\left(\mathrm{kg} / \mathrm{m}^{3}\right)\end{array}$ & $\begin{array}{c}\text { Water } \\
\left(\mathrm{kg} / \mathrm{m}^{3}\right)\end{array}$ & $\begin{array}{c}\text { Sand } \\
\left(\mathbf{k g} / \mathrm{m}^{3}\right)\end{array}$ & $\begin{array}{c}\text { Coarse } \\
\text { aggregate } \\
\left(\mathbf{k g} / \mathbf{m}^{3}\right)\end{array}$ & $\begin{array}{c}\text { Air-entraining } \\
\text { dgent } \\
\left(\mathbf{k g} / \mathbf{m}^{3}\right)\end{array}$ & $\begin{array}{c}\text { Air } \\
\text { content } \\
(\%)\end{array}$ \\
\hline $\mathrm{C} 50$ & 0.32 & 42.5 & 526.00 & 168.30 & 520.00 & 1154.80 & & \\
$\mathrm{C} 40$ & 0.36 & 42.5 & 467.60 & 166.00 & 568.20 & 1148.00 & 1.30 & $5.5 \sim 6.5$ \\
C30 & 0.40 & 42.5 & 412.67 & 164.30 & 586.83 & 1186.00 & 1.17 & $5.5 \sim 6.5$ \\
C25 & 0.40 & 32.5 & 356.00 & 141.00 & 615.20 & 1188.00 & 1.03 & $5.5 \sim 6.5$ \\
C20 & 0.40 & 32.5 & 339.00 & 133.80 & 642.00 & 1185.20 & 0.89 & $5.5 \sim 6.5$ \\
& & & & & & & 0.85 & $5.5 \sim 6.5$ \\
\hline
\end{tabular}

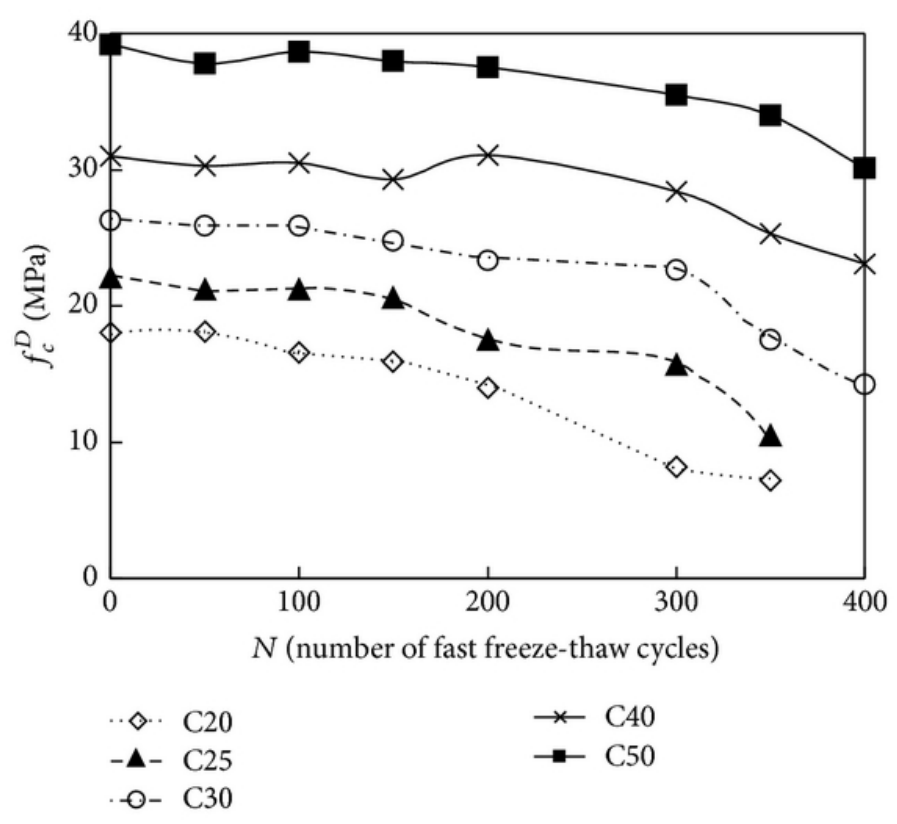

Figure 2.2.8: Number of freeze-thaw cycles vs. compressive strength (Shang, Cao, Wang, 2014)

Figure 2.2.8 shows that as the number of freeze-thaw cycles increase, the compressive strength of the concrete decreases. It also illustrates that mixes $\mathrm{C} 20, \mathrm{C} 25$, and $\mathrm{C} 30$, which all contain the highest water-cement ratios at 0.40 , have the lowest compressive strength. In addition, they also seem to decrease in strength faster as the number of cycles increase. The mix that maintains the highest level of compressive strength is the mixture with the lowest water-cement ratio, thus having the most cement and most air entrainment content.

\subsubsection{Scaling Resistance}

The test method to determine scaling resistance of concrete surfaces exposed to deicing chemicals is found in ASTM C672 (2012). The test method includes exposing concrete samples to salt 
solutions on one side of the specimen and heat flow on the other. The sample is put through freezethaw cycles and the materials that have detached due to scaling is collected and weighed (Ronning, 2001). The percentage loss by mass is recorded to determine the severity of scaling due to deicing chemicals under freezing and thawing conditions. Figure 2.2.9 shows data obtained in a lab, comparing different air void contents and their effects on scaling of concrete. The scaling due to freezing and thawing is incredibly high when minimal air content is provided. Once at least 3\% air content is present, the amount of scaling drops immensely with almost no scaling at $6 \%$ air content. Thus, under extreme weather conditions, it is ideal to have close to $6 \%$ air voids in your concrete.

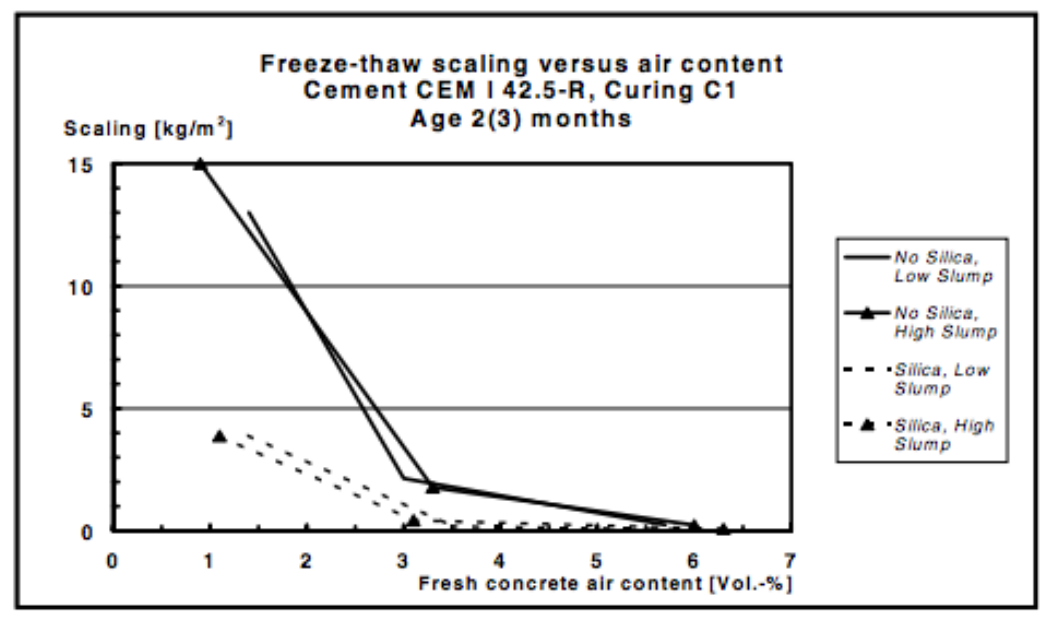

Figure 2.2.9: Air content vs. scaling due to freeze-thaw cycles (Ronning, 2001)

\subsection{Supplementary Cementing Materials}

As a major contributor to $\mathrm{CO}_{2}$ emissions, the production of Portland cement has been recognized to be a great environmental issue. Thus, the construction industry has discovered an innovative way to replace a portion of cement in concrete mixtures in order to reduce its negative environmental effect. The use of supplementary cementing materials (SCM) has become widely popular in the concrete industry as a substitute for Portland cement. Thus, the study of various 
SCM at different proportions in concrete arises (Lothenbach, Scrivener, and Hooton, 2011). Three of the most commonly used SCM are silica fume, blast-furnace slag and fly ash. Each contains diverse properties that effect concrete in various ways.

\subsubsection{Fly Ash}

Fly ash is a pozzolanic material classified as an SCM. This fine material is a product of the combustion of coal. Its physical properties of fine, spherical particles are the reason why fly ash produces workable concrete (Supplementary Cementitious Materials, 2006). The chemical property of fly ash includes its reaction with calcium hydroxide, which is a by-product of the hydration process in cement (Supplementary Cementitious Materials, 2006). In addition to its benefit to the environment, fly ash also reduces the heat of hydration in concrete, reduces it permeability, provides higher ultimate strength, and increases the concretes resistance to alkali silica reaction. Due to its chemical property of reacting with alkalis in concrete, the use of fly ash reduces the available alkalis in the concrete, which in turn reduces the amount of available alkalis that can react with silica found in the aggregate (Siddique and Khan, 2011). The addition of fine, pozzolanic materials, such as fly ash and silica fume, neutralizes the alkalinity of the cement, which is greatly beneficial in mitigating ASR (Siddique and Khan, 2011).

\subsubsection{Silica Fume}

Silica Fume is another widely used SCM known to increase the durability of concrete, reduce its permeability and calcium hydroxide content, and yields a higher resistance to sulfate attack (Siddique and Khan, 2011). It is the result of the production of silicon or alloys containing silica in electric arc furnaces. The many advantages associated with the use of silica fume include a high early compressive strength, high tensile and flexural strength, increased durability and a reduction 
in expansion due to ASR. According to Douglas Hooton, a professor from University of Toronto, expansion in mortar bars made with high-alkali cement was inversely proportional to the amount of silica fume used in the place of Portland cement (Siddique and Khan, 2006). Thus, it was concluded that the use of silica fume reduced the expansion in mortar bars made with high-alkali cement.

\subsubsection{Slag}

Ground granulated blast furnace slag is an SCM that is produced in blast furnaces that are used to create iron. Its physical properties include small, fine particles that result in a "glassy" material, its low specific gravity and its beige to brown colour. Some of its advantages include improved workability, increased pumpability and strength, and its high resistance to ASR. Slag is widely known to effectively mitigate ASR in concrete for multiple reasons. Similar to fly ash, slag greatly reduces the alkalinity of the concrete by bonding with the available alkalis. This results in a decrease of available alkalis and thus, decreases the opportunity for silica to react with the alkalis to produce ASR. Slag also reduces the mobility of the alkalis as well as the free lime. The reduction of free lime in concrete is directly proportional to the reduction of ASR in concrete; this is due to the fact that ASR is a calcium-based reaction. With the presence of free lime in the concrete, its calcium is able to react with the available silica and hydrogen to form the calcium-silica-hydrate gel that is ASR. Thus, a reduction in free lime is a reduction in ASR (Siddique and Khan, 2006).

\subsection{Recycled Concrete Aggregate}

As the construction industry has become increasingly environmentally conscious, new and innovative ways to deal with environmental issues have been proposed. There are three major issues present in the concrete industry, which are: depleting natural aggregates, decreasing landfill space and $\mathrm{CO}_{2}$ emissions. In order to address these issues, the industry has come with some 
initiative including the use of recycled concrete aggregate (RCA). RCA is can be used as a partial substitute for aggregate in concrete, which directly reduces the amount of natural aggregate used. RCA is taken from existing structures that are demolished. Once the demolished structure is crushed and reduced to small, coarse aggregate-like sizes, the RCA can be used in new concrete structures as a partial replacement of aggregate (Radonjanin et al., 2013). The environmental benefits associated with using RCA include the use of waste concrete, which in turn, reduces the total volume of landfill wastes, as well as a reduction of natural aggregate consumption or depletion (Radonjanin et al., 2013). The composition of RCA is a major factor in the new concrete's performance. Due to the fact that a portion of the aggregate is replaced with RCA, additional cement paste is introduced in the mixture. Thus, as opposed to the traditional concrete mixture where the cement paste bonds with the aggregate, the new cement paste bonds with the old cement paste forming two Interfacial Transition Zones (ITZ) rather than one in case of concrete with virgin aggregate. Interfacial Transition Zone is a zone of porous and relatively weak phases at the interface between aggregate and paste. This reduces the durability and strength of the concrete. In addition, the cumulative volume of old and new paste increases the bulk volume of paste which comes with some negative impacts including increased shrinkage. Various testing has been conducted to determine and compare the physical properties of RCA to traditional concrete. It can be concluded that concrete with RCA has higher absorption when compared to traditional concrete due to the increased cement paste. In addition, as the RCA content increases, the slump of the concrete decreases, the compressive strength decreases, and the durability decreases (Radonjanin et al., 2013). In order to achieve the best possible performance of RCA concrete, proper testing should be conducted on the RCA to determine its properties and how these properties can affect the new concrete. It is important to know what aggregate was used in the 
original structure and what deterioration has occurred to the structure. Properties such as the durability of the aggregate and whether or not the RCA is reactive is very important to know in order to determine the performance and quality of the new concrete structure.

\subsubsection{Reactivity of RCA}

When recycled materials are considered for use in concrete, engineers and owners must perform the necessary testing to determine the composition and reactivity of the RCA to predict how it will behave in the new concrete. For example, testing the composition of RCA is highly important to determine if the material is reactive, which can be detrimental to new concrete. It has been determined that new concrete with RCA that has been affected by ASR in its previous structure produces more expansion when compared to concrete with virgin reactive aggregate. Various lab tests were conducted using Spratt-RCA and Spratt virgin aggregate (Shehata et al., 2010, 2012), which are highly reactive aggregates, in order to determine and compare the expansions results between the two and understand how ASR-affected RCA affects new concrete. The series of tests included the concrete prism test (CPT) and the concrete microbar test (CMBT). The results of the CPT test show that after one year, concrete cast with $100 \%$ Spratt-RCA expanded only slightly higher than that of the virgin Spratt aggregate, concluding that the use of Spratt-RCA in concrete is feasible with the proper use of SCM (Shehata et al., 2010, 2012). As more reactive aggregates get tested as an RCA, it will be easier to understand the difference in expansion between the RCA and its virgin aggregate. For instance, if a structure has undergone variable deterioration, it is important to determine if the level of deterioration that has occurred on the previous structure will affect the expansion or deterioration in the new structure. In addition, it is important to determine if each reactive RCA expands higher, lower or similarly to their respective virgin aggregate. This study investigates the reactivity of RCA containing moderately reactive gravel and the same virgin 
aggregate to understand if they follow the same trend as previously found for RCA containing highly reactive aggregate.

\subsection{Sudbury Bridge}

A bridge built in Sudbury, Ontario over 20 years ago was built with an aggregate found in a local quarry nearby, now known as Sudbury aggregate. This gravel aggregate was used because the quarry was local and the aggregate was easily accessible. What was not known at the time was that this aggregate is highly susceptible to ASR. The bridge has since been removed and replaced due to the level of deterioration that had occurred to the bridge. The figures below show a variety of cracking and deterioration at the surface of various highway road barriers on the bridge.

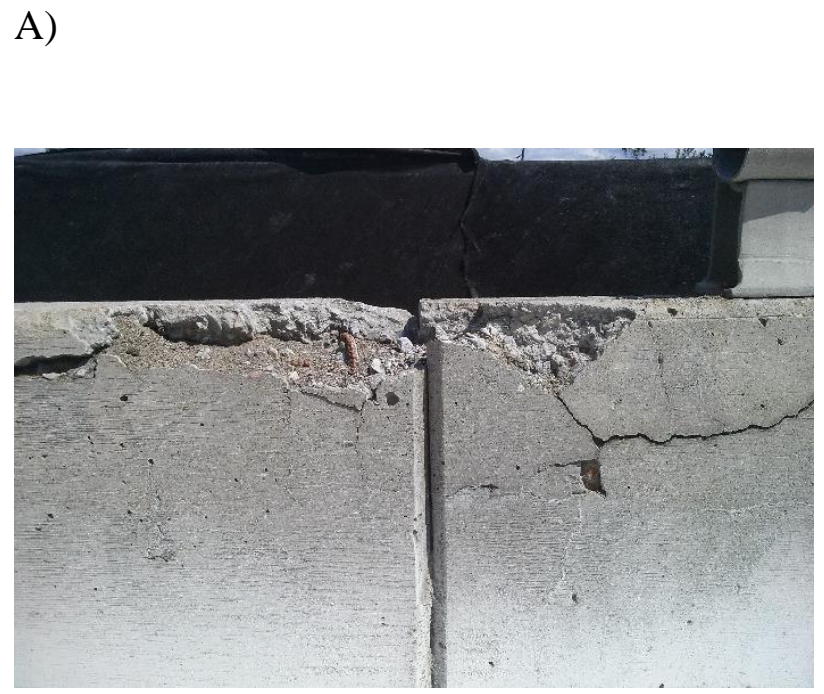

Cracking at joint of road barriers
B)

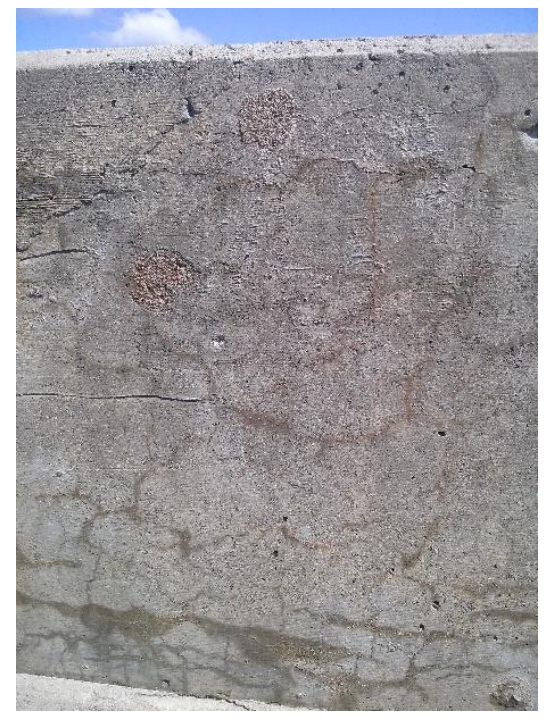

Map cracking on back of barrier 
C)

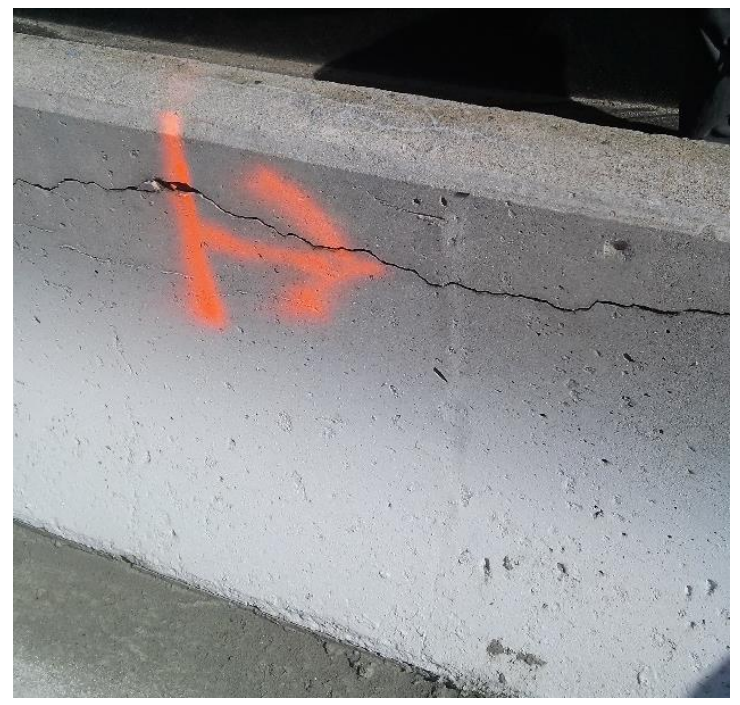

Horizontal cracking at face of barrier
D)

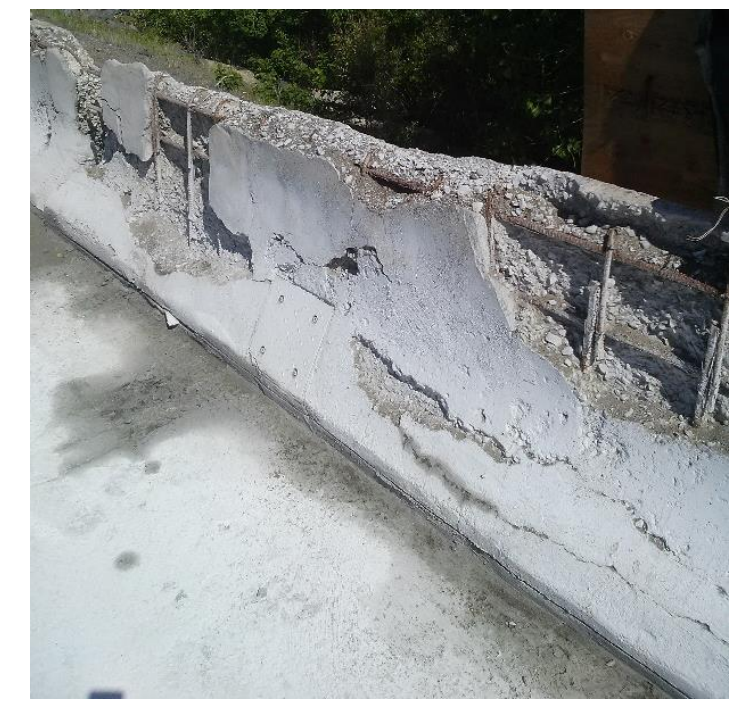

High level of deterioration at face of barriers

Figure 2.4.1: Deterioration of highway road barriers in Sudbury, Ontario

The cracks and deterioration shown in Figure 2.4.1 is a result of the combined effects of freezing and thawing deterioration and alkali-silica reaction. These two concrete deterioration mechanisms share similarities in that the visual cracking and deterioration caused by them is very similar. When the two act together, a variety of levels of deterioration become apparent due to the orientation of the concrete. That is, concrete exposed to more severe weather, such as the surface facing the sun or concrete exposed to more water, will deteriorate at a quicker rate due to the stimulation of the deterioration mode. Other, less likely, factors could include different batches of mixes (nonuniformity) or improper finishing of the concrete. This can help explain the different levels of deterioration shown in Figures 2.4.1. It is likely that parts of the structure showing far more deterioration (such as Figure 2.4.1-D) were exposed to more severe weather conditions than some other surfaces (shown in Figure 2.4.1-C), which aided in expediting the deterioration. The joint crack shown in Figure 2.4.1-A is a result of expansion within the two barriers, causing a pressure 
buildup, resulting in a crack at the weakest point of the barriers. In Figure 2.4.1-B, map cracking is apparent on the backside of the barrier due to the formation of the rebar within the barrier.

\subsubsection{Bridge Condition Surveys}

Detailed bridge condition surveys were completed on the Sudbury Bridge through the Ministry of Transportation (MTO) in August of 2011. The two locations of importance to this research are the North-West bridge ramp, which is where the low deteriorating barriers were obtained from, and the East-North bridge ramp, which is where the high deteriorating barriers were obtained from. Figure 2.4.2 shows pictures taken from the bridge condition survey reports of each bridge ramp conducted by the MTO. Figure 2.4.2-A shows a low deteriorating barrier from the North-West ramp and Figure 2.4.2-B shows a high deteriorating barrier from the East-North ramp.

A)

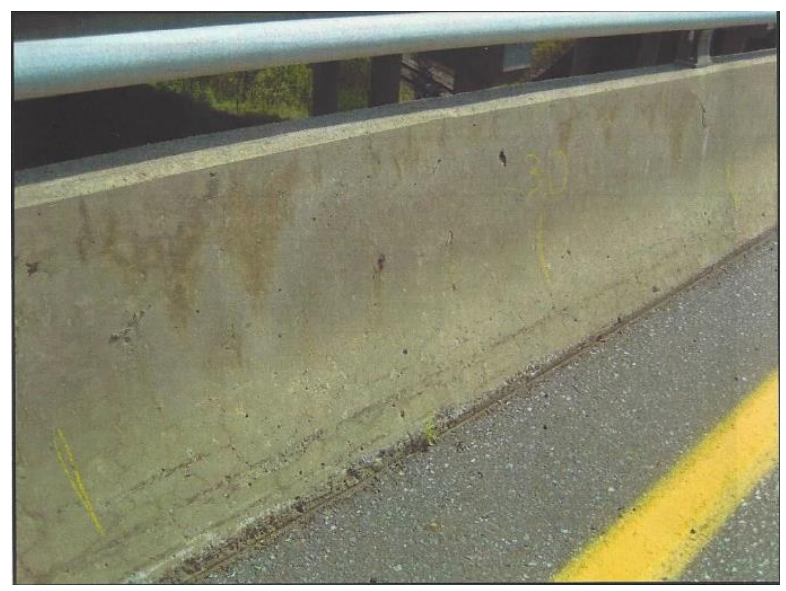

Low deteriorating road barrier (East-North ramp)
B)

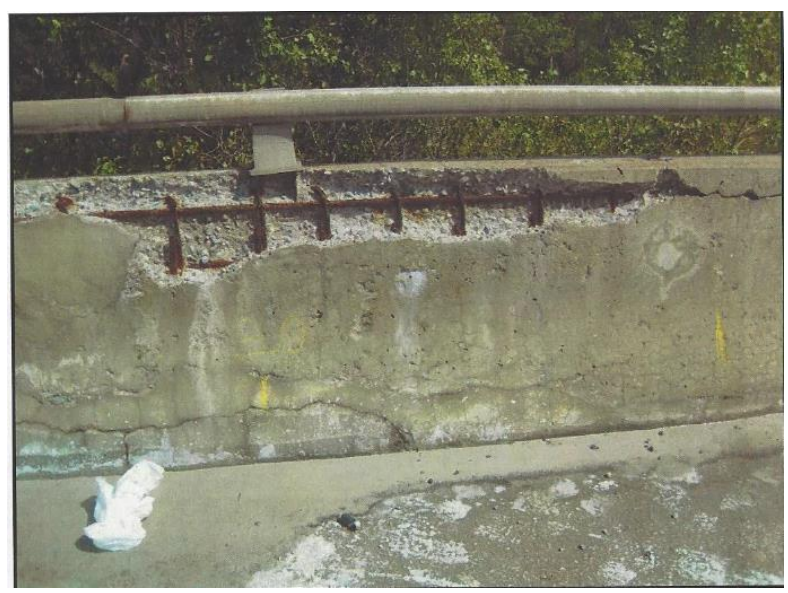

High deteriorating road barrier (North-West ramp)

Figure 2.4.2: Low and high deteriorating road barriers from bridge condition survey conducted by MTO in 2011

The bridge condition survey of the North-West ramp concluded that the concrete barrier walls were in fair condition. It suggested that the interior faces consisted of localized spalling, 
delamination, exposed corroded reinforced steel, medium scaling, and wide cracking. The exterior faces were considered to be in good condition with minor longitudinal cracking, medium scaling, and localized small spalls.

The East-North ramp bridge condition survey concluded that the barrier walls were in poor to fair condition. The interior faces were considered to have localized spalling, delamination, exposed and corroded reinforced steel, and extensive wide longitudinal cracking. The exterior faces were considered to be in fair to good condition, with minor longitudinal cracking and localized small spalls. 


\section{Chapter 3: Experimental Procedures and Material Details}

\subsection{Scope of Work}

This thesis will examine virgin Sudbury aggregate and Sudbury RCA obtained from a bridge built in Sudbury over 20 years ago that had deteriorated from both freeze-thaw deterioration and ASR. The research conducted for this thesis includes a variety of testing to compare virgin Sudbury aggregate with Sudbury RCA, including providing various methods of mitigation. Mitigation techniques were used in both newly casted concrete containing virgin aggregate and RCA as well as existing concrete containing Sudbury aggregate. This was done in an effort to better understand the reactions that have already occurred, whether or not they will continue to occur, and any new reactions that will begin to occur in the new concrete containing RCA. In order to do so, the Ministry of Transportation (MTO), has provided virgin Sudbury aggregate, RCA from the demolished Sudbury bridge, and road barriers from the bridge. The road barriers were hand selected based on the level of deterioration that had occurred in them and were separated into two categories - high deteriorated and low deteriorated. Along with testing the expansion in the road barriers, cores were extracted - from both high and low deteriorated barriers - and kept separate. Since the RCA sent by the MTO from Sudbury was not hand selected, RCA was also produced from the road barriers of high and low deterioration and also kept separate. This was done to determine if the level of previous deterioration has an effect on the deterioration that will occur in the new structure. Therefore, RCA obtained from high deteriorated road barriers will be referred to as RCA-H, RCA obtained from low deteriorated road barriers will be referred to as RCA-L, and RCA obtained randomly from the bridge will be referred to as mixed RCA or RCA-M. Virgin Sudbury aggregate, along with RCA-H and RCA-L was used to produce concrete prisms and cylinders in order to correlate results obtained from the extracted cores and the road barriers. In an 
effort to examine mitigation methods on structures already affected by ASR, a waterproofing agent, known as a silane-base sealer, was used on these samples. Ultimately, the objective is to draw a correlation between the results of the prisms and cores (through the results of the cylinders) and a correlation between the cores in the lab and the road barriers in the field. The mixed RCA was used in comparison to virgin Sudbury aggregate for concrete prisms, microbars, and mortar bars. This was done to determine if the concrete microbar test (CMBT) and the accelerated mortar bar test (AMBT) could accurately predict the expansion obtained by the concrete prism test (CPT). Various amounts and combinations of supplementary cementing materials (SCM) were used as a mitigation technique for these samples to determine what is necessary to effectively mitigate expansion of samples containing virgin Sudbury aggregate in comparison to samples containing Sudbury RCA. SCM were also used to verify the validity of the CMBT and AMBT in predicting the CPT results for Sudbury aggregate and Sudbury RCA given a variety of SCM blends. The results obtained for virgin Sudbury aggregate and Sudbury RCA from the CPT and AMBT can be compared with the results obtain for Spratt and Spratt RCA from Shehata et al. to better understand the trends associated with the virgin aggregate and it as an RCA.

It is the goal that, through the findings in this research, society becomes more accepting of the use of RCA in new structures, including RCA that has been previously affected by ASR. Although this research only examines samples consisting of 100\% RCA, which is not realistic to occur anytime in the near future, it is the first step towards partial use of RCA with moderately reactive aggregate in new structures. Thus, further research should be conducted through the CPT, AMBT, and CMBT tests using partial RCA replacement along with SCM in samples containing both virgin Sudbury aggregate and Sudbury RCA. 


\subsubsection{Test Development}

As mentioned, expansion testing due to ASR was measured on casted concrete cylinders and extracted cores. Because testing these two samples for ASR expansion is not standard practice, test methods needed to be developed to accommodate for their testing. For example, holes in the top and bottom of the cores needed to be drilled in order to imbed measuring studs and cylinders needed to be casted with measuring studs placed in them. For the cores, an apparatus was manufactured in order to accurately drill and place studs into the centre of the faces. For the cylinders, moulds were modified to allow for measuring studs to be cast into the faces. ASR testing of cylinders and cores were done in the lab in an effort to compare data from the lab to the field.

\subsubsection{Extracted Cores}

The cores were extracted from high and low deteriorating road barriers using a coring drill. Six cores from high deteriorating road barriers and six cores from low deteriorating road barriers that range between $170 \mathrm{~mm}$ and $230 \mathrm{~mm}$ in length were extracted and placed in sealed bags until they were ready for testing. The cores were limited to this range in length so that they can accurately be compared to a concrete cylinder, which has a length of $200 \mathrm{~mm}$. Before testing began, an apparatus was made using 1 inch by 1 inch steel tubing, 1 inch angle iron, and set screws to drill holes into the top and bottom of the cores. These holes were drilled $3 / 4$ of an inch deep and $3 / 8$ in

diameter using a drill press, as shown in Figure 3.1.1-A. Studs that were 1 inch in length and $1 / 4$ inch in diameter were placed into the drilled holes and secured with non-shrink grout, shown in Figure 3.1.1-B. 
A)

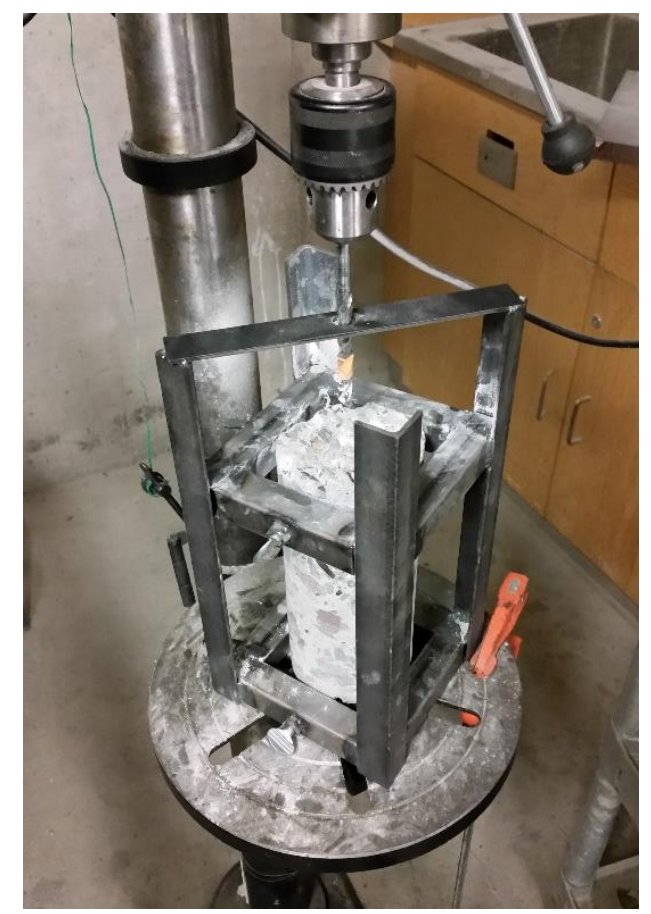

Drilling holes into face of cores
B)

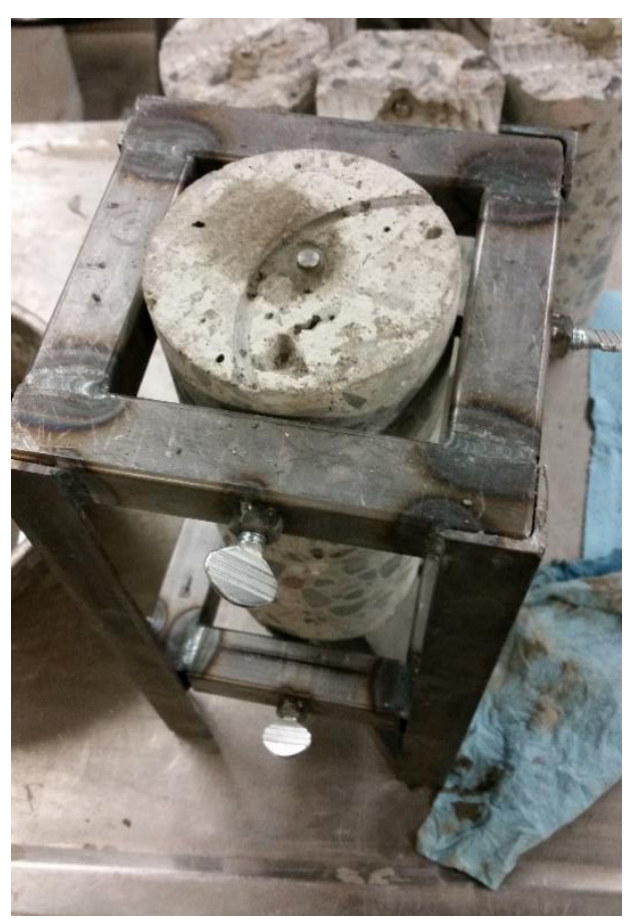

Stud placement using non-shrink grout

\section{Figure 3.1.1: Core drilling and measuring stud placement apparatus}

Two layers of silane-sealer application were done on three high deteriorated cores and three low deteriorated cores. As per the guidelines of the silane-sealer, they were left out to dry for 7 days before testing began. The silane-sealer application process can be seen in Figure 3.1.2 below. In order to keep results consistent, the cores that did not have the silane-sealer applied - three high and three low deteriorated cores - also sat for 7 days at room temperature. A zero reading was taken of the cores after these 7 days, in which they were completely dry, and placed in the heat room at $38{ }^{\circ} \mathrm{C}$ as per CSA A23.2-09 14A (2009) standard for testing concrete prisms for ASR. 


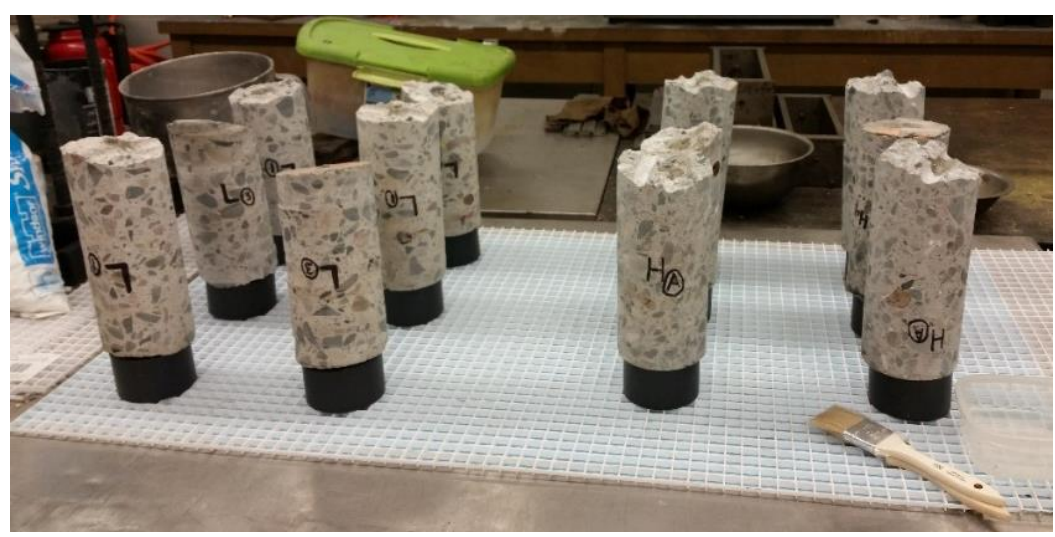

Figure 3.1.2: Application of silane-sealer on cores

\subsubsection{Concrete Cylinders}

Cylinder moulds were modified by using a standard concrete cylinder mould (100 mm in diameter and $200 \mathrm{~mm}$ in length) and drilling a 5/16 of an inch hole through the middle of the bottom of the mould. Then, two squares were cut at $120 \mathrm{~mm}$ by $120 \mathrm{~mm}$ in $3 / 4$ inch plywood. A $3 / 8$ inch hole was drilled through the centre and a female threaded binding barrel was place in the hole. Using Autocad drawings and a laser cutter, these same $120 \mathrm{~mm}$ by $120 \mathrm{~mm}$ squares were cut out of acrylic plastic, with $100 \mathrm{~mm}$ diameter circles cut out of them. The acrylic squares was screwed down to the wooden blocks to complete the mould. Then, a stud was created using $1 \frac{1 / 4}{4} \mathrm{inch}$ long by $1 / 4$ inch diameter stainless steel hex head screws, with the ends sanded to a flat surface using a drill press and a belt sander and screwed into the female threaded binding barrels, shown in Figure 3.1.3-A. The blocks sit on the top and bottom of the concrete cylinder mold after casting, and elastics are used to hold them tight together until demoulding, shown in Figure 3.1.3-B. Six of these molds were created since each mix contains three cylinders with the silane-sealer and three cylinders without. 
A)

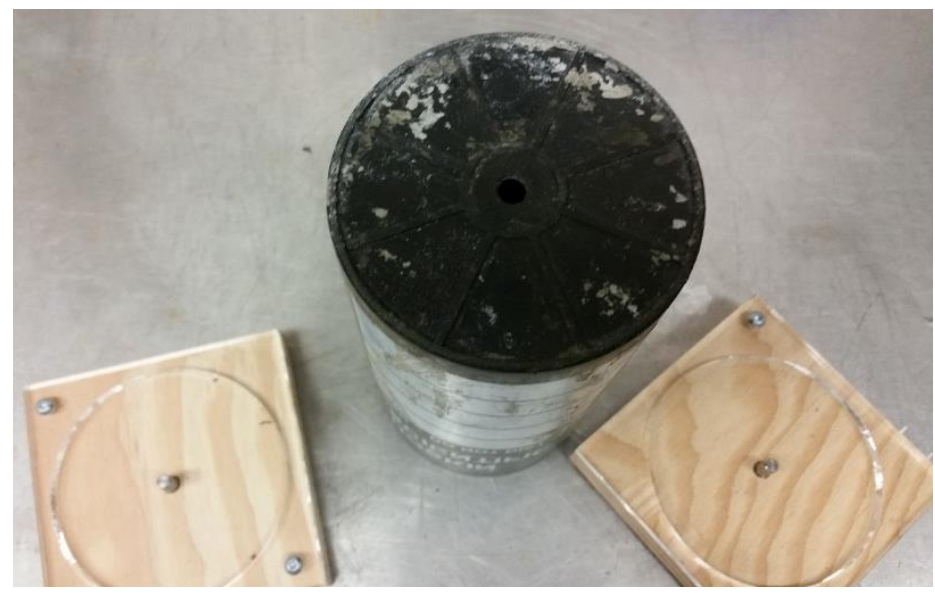

Parts required to test cylinders for expansion
B)

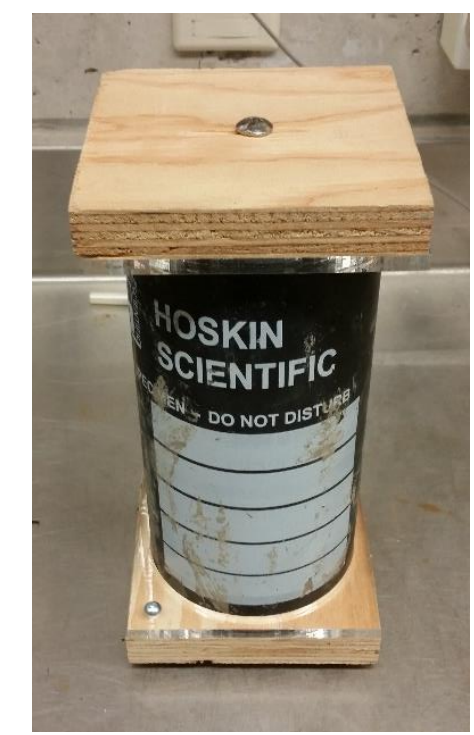

Completed Cylinder Mould

Figure 3.1.3: Cylinder mould for expansion testing

The purpose of creating cylinders to be tested for expansion is to relate the expansion measured in the concrete prisms to the extracted cores. Since the extracted cores are of similar shape and size as the cylinders, the expansion can be related between the two and then compared to the standardized concrete prism test. This will allow for a better understanding of the expansion results obtained in the cores.

The testing procedure of the cylinders were done similar to that of the extracted cores in order to keep results consistent. There were two difference that occurred: (1) the zero reading was taken within an hour of being demoulded, therefore in a saturated state and (2) once the initial reading was taken, the cylinders were placed in a bucket over water at room temperature for 14 days for curing. This method was used for curing so that the samples would not become wet in order to reduce expansion and leaching before testing. After curing, silane-sealer was applied over two days, allowed to sit for 7 days to dry, and placed in the heat room at $38{ }^{\circ} \mathrm{C}$ as per the CSA A23.2-09 14A (2009). 


\subsubsection{Road Barriers}

Four road barriers ( 2 high and 2 low deteriorated barriers) had holes drilled into them using a hammer drill in order to imbed measuring studs. The holes were drilled with a diameter of $5 / 8$ " and had $1 / 4$ inch diameter, inch and a half long steel dowels placed in them using non-shrink grout. The dowels extended beyond the surface of the barrier by about $5 \mathrm{~mm}$ for ease of measurement and had a conical hole drilled in the centre of the top face using an end drill to allow for measurement using the Whittemore Strain Gauge. The centre of the dowels were positioned 20 inches away from each other, which is the centre of the Whittemore's measuring range. Measuring the expansion of the road barriers on site will allow for a comparison to be made between expansion found in the lab on the prisms, cylinders, and cores to these barriers in the field. After obtaining a correlation between expansion of the samples in the lab, the goal is to be able to compare the expansion of the cores to the expansion of the barriers to see how the lab affects expansion of similar samples.

After obtaining data for one year on the road barriers, 1 high and 1 low deteriorated barrier has had a silane-based sealer applied to them. The sealer was applied in two coatings over two days. Since the barriers are placed in a manner of which the faces of the barrier are facing up, the sealer was applied to the face and sides of the barriers. The barriers were then covered with vapour barrier for 7 days to dry, before being re-exposed to normal weather conditions. The barriers will continue to be monitored to determine both the difference in expansion between lab and field results as well as the effects of silane-based sealers on previously deteriorated structures. 


\subsubsection{Sample Preparation}

There were a variety of samples tested for expansion due to ASR in the lab. Each type of sample consisted of 3 specimens, making up a 'set' of samples. Each sample that was cast in the lab had a measuring, or gauge, stud placed in each end. The samples were made in accordance with ASTM C192 - Standard Practice for Making and Curing Concrete Test Specimens in the Laboratory. Any samples obtained outside of the lab were embedded with a measuring stud by drilling a hole and being placed with non-shrink grout. The samples cast in the lab include mortar bars, microbars, prisms, and cylinders. The mortar bars, microbars, and prisms were all measured using a length comparator and compared to an invar bar. The invar bar was calibrated against a reference bar and all measurements were adjusted for the difference. The expansion measurements for cylinder and cores were taken using outside micrometers because the length of the samples were too short to be taken with the length comparator. The expansion measurements for the road barriers were taken with the Whittemore Strain Gauge, which are being tested in the field for combined freeze-thaw deterioration and ASR. All measurements were taken as per ASTM C490 - Standard Practice for Use of Apparatus for the Determination of Length Change of Hardened Cement Paste, Mortar, and Concrete.

\subsubsection{Mortar Bars}

CSA A23.2-25A - Test method for deterioration of alkali-silica reactive aggregate by accelerated expansion of mortar bars was followed when preparing mortar bars. To begin, a representative sample of $21 \mathrm{~kg}$, which includes $7 \mathrm{~kg}$ of each fraction $5 \mathrm{~mm}$ to $20 \mathrm{~mm}$, was taken to ensure a representative portion of the original coarse sample was taken. The $21 \mathrm{~kg}$ was then crushed using a small jaw crusher until all materials passed the $5 \mathrm{~mm}$ sieve. The material was then further crushed using a disk pulveriser until the proper fractions of each size was obtained. When crushing the 
RCA, it was ensured that all $21 \mathrm{~kg}$ was crushed into the necessary fractions so that a representative sample was used, as proposed by Adams et al. (2013). This was done because if the aggregate was just crushed until the amount needed was obtained, a representative sample would not have been obtained because when crushing RCA, the cement paste separates first. This is due to its much lower durability compared to the aggregate, thus crushing the whole batch prevents a cementdominant mix. Figures 3.1.4 show the crushed fractions for Sudbury RCA as described.

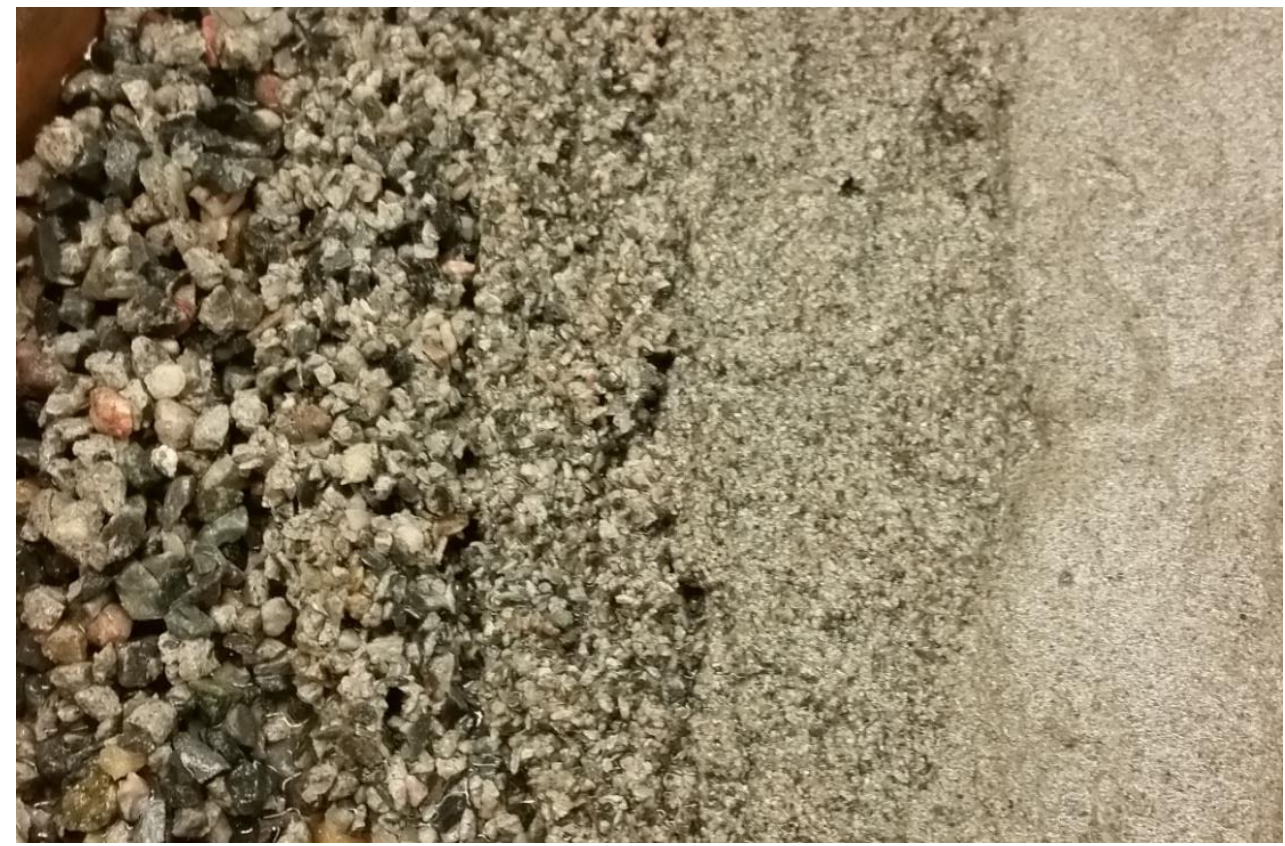

Figure 3.1.4: Graded RCA used for AMBT starting from retained on $2.36 \mathrm{~mm}$ (left) to retained on $0.150 \mathrm{~mm}$ (right)

In Figure 3.1.4, a representative portion of both the original aggregate and residual mortar can be seen from the RCA rubble that was crushed. Once the proper portions were obtained, each size of aggregate was washed over the sieve and dried before casting. The washing procedure used on the Sudbury RCA was different than that of the virgin aggregate. When washing the RCA, the procedures followed were those outlined by Adams et al. (2013). The washing procedure is as follows: 
1. Sieve each aggregate, and keep separated according to each fraction retained on sieve sizes;

2. Measure out about $3.5 \mathrm{lbs}$. (1600 g) of material onto a fine sieve;

3. Wash aggregate using a rubber hose with a fanned-spray hose nozzle for the following times for each retained on sieve size:

○ \#8 Sieve: 3 minutes 30 seconds

○ \#16 Sieve: 5 minutes

- \#30 Sieve: 6 minutes

○ \#50 Sieve: 7 minutes

○ \#100 Sieve: 8 minutes;

4. Place aggregate into a $230^{\circ} \mathrm{F}\left(110^{\circ} \mathrm{C}\right)$ oven to dry for $24 \pm 2$ hours before using.

After the aggregate is dried, the samples can be prepared. The size of the bars are $25 \mathrm{~mm}$ x $25 \mathrm{~mm}$ x $285 \mathrm{~mm}$ with a gauge length of $250 \mathrm{~mm}$. When casting the virgin aggregate, no modifications were made to the procedures outlined by CSA A23.2-25A (2009). The interior of the moulds were covered in a release agent and the measuring studs were placed in the moulds. The mortar, made of $440 \mathrm{~g}$ cementing materials (including SCM), $990 \mathrm{~g}$ graded aggregate, and $220 \mathrm{~g}$ water, was mixed in accordance with the requirements set out by ASTM C305 - Standard Practice for Mechanical Mixing of Hydraulic Cement Pastes and Mortars of Plastic Consistency. When casting the RCA aggregate, a modified mix procedure was used. The modified procedure allowed for aggregate absorption corrections due to the large absorption characteristics of RCA. The following is the mix procedure used for casting mortar bars containing RCA, obtained from Adams et al. (2013):

1. After washing and drying the aggregates, soak in the mixing water which is corrected for $95 \%$ of the aggregate absorption, for a period of 30 minutes;

2. Mix soaked aggregate for 30 seconds in mixer on low speed;

3. Slowly add cement over a 30 second period while mixing on low speed;

4. Stop the mixer and let the mortar stand for 1 minute and 30 seconds. During the first 15 seconds of this rest period, scrape down into the mixture any mortar that may have collected on the side of the bowl; then cover the bowl with a lid;

5. Finish mixing the mortar on medium speed for 1 minute;

6. Cast specimens. 
The specimens were then left in the moisture room for 24 hours before being demoulded. The specimens had their initial lengths measured and were placed in a container containing room temperature water that saw the samples completely submerged in water. The containers were sealed and placed in an oven at $80{ }^{\circ} \mathrm{C}$ for 24 hours. After 24 hours, the bars were measured and the zero reading was obtained. The bars were then transferred to sealed containers containing a $1 \mathrm{~N}$ solution of sodium hydroxide preheated to $80{ }^{\circ} \mathrm{C}$ and put back in the oven. Subsequent measurements were taken at day 3, day 7, day 10, day 14, day 21, and day 28 for all mortar bar specimens.

\subsubsection{Microbars}

The concrete microbars were prepared as per Rilem AAR-5 - Rapid preliminary screening test for carbonate aggregates (2005). In doing so, aggregate was crushed using a small jaw crusher to obtain two aggregate sizes, between $4.75 \mathrm{~mm}-9.5 \mathrm{~mm}$ and between $9.5 \mathrm{~mm}-12.5 \mathrm{~mm}$. The two sizes of aggregates were used to compare expansion of samples containing $4.75 \mathrm{~mm}-9.5 \mathrm{~mm}$ aggregate versus $9.5 \mathrm{~mm}-12.5 \mathrm{~mm}$ aggregate. When preparing the samples using the virgin Sudbury aggregate, the aggregate was washed over a sieve, similar to that of the virgin aggregate for the mortar bars. On the contrary, the RCA was not washed in an effort to reduce the leaching of alkalis. Once the aggregate was dried, $1800 \mathrm{~g}$ of cementing materials (including SCM), $1800 \mathrm{~g}$ of aggregate and $580 \mathrm{~g}$ of water. The water was also corrected for absorption for both aggregate types. In addition, the sodium hydroxide equivalent $\left(\mathrm{Na}_{2} \mathrm{O}_{e}\right)$ of the concrete is required to be brought up to $1.5 \%$ by mass of cement. Thus, if the cement contains $0.99 \% \mathrm{Na}_{2} \mathrm{O}_{e}$, an addition $0.51 \%$ by mass of cement is added to the mixing water. The mixing procedure for pastas, found in ASTM C305 (2014), was followed. After mixing the cement and water, the aggregate was mixed in by hand until the mixture was consistent and all aggregate was fully coated. The mix was then 
placed in moulds, which were coated with a release agent and measuring studs placed in, of size $40 \mathrm{~mm}$ x $40 \mathrm{~mm}$ x $285 \mathrm{~mm}$ with a $250 \mathrm{~mm}$ gauge length and placed in the moisture room to cure for 24 hours. Similar to that of the mortar bars, the initial reading was taken after demoulding, the specimens were placed in containers containing water and into the $80{ }^{\circ} \mathrm{C}$ oven for 24 hours, the zero readings was taken, and the specimens were then placed into a preheated solution of $1 \mathrm{~N}$ of sodium hydroxide and back into the oven. Readings were then taken weekly for 8 weeks.

\subsubsection{Concrete Prisms and Cylinders}

The procedures followed for casting the concrete prisms and cylinders follow the guidelines set out in CSA A23.2-14A - Potential expansivity of aggregates (procedure for length change due to alkali-aggregate reaction in concrete prisms at $38^{\circ} \mathrm{C}$ ). The aggregate was sieved into three equal portions of aggregate from $19.5 \mathrm{~mm}-13.5 \mathrm{~mm}, 13.5 \mathrm{~mm}-9.5 \mathrm{~mm}$, and $9.5 \mathrm{~mm}-4.75 \mathrm{~mm}$. The mixing portions consisted of $420 \mathrm{~kg} / \mathrm{m}^{3}$ (unless otherwise noted), a coarse to fine aggregate ratio of 60:40 by mass, a water-cementing ratio of 0.45 (along with absorption corrections), and an alkali content raised to $1.25 \% \mathrm{Na}_{2} \mathrm{O}_{\text {e. }}$. Once everything was ready, the specimens were mixed using the following mixing procedure:

1. Add aggregate and mix for 1 minute

2. Add $1 / 2$ of water while mixing and mix for 1 minute

3. Rest for 30 seconds

4. Add cement and remaining water over 30 seconds while mixing

5. Mix for 1 minute

6. Rest for $2 \mathrm{~min}$

7. Mix for $2 \mathrm{~min}$

8. Rest for $2 \mathrm{~min}$

9. Mix for $2 \mathrm{~min}$ 
After mixing, the mix was placed and rodded into moulds that were coated with release agents and have studs placed in. The prism moulds $(75 \mathrm{~mm}$ x $75 \mathrm{~mm} \times 285 \mathrm{~mm}$ with a gauge length of 250 $\mathrm{mm}$ ) were casted horizontally and in three layers, ensuring proper compaction, especially around the measuring stud, and finished flat - just as the mortar bars and microbars. The cylinder moulds (100 mm diameter by $200 \mathrm{~mm}$ length) were casted similarly, with the only difference being that they were cast vertically. The samples were then placed in the moisture room for 24 hours, after which they were demoulded and an initial zero reading was taken immediately. After the initial reading was taken, two different procedures were used. The first method, which consists of following CSA A23.2-14A (2009), was done for the set of prisms used to compare different levels of SCM (that would also be compared to the microbars and mortar bars). This consists of placing them in sealed buckets lined with damp cloth and raised over water, vertically, in a heat room controlled to $38{ }^{\circ} \mathrm{C}$. In the second method, which consists of comparing prisms, cylinders, and extracted cores in addition with the use of a silane-based sealer, a slight modification was made. Instead of immediately placing the sealed buckets into the controlled heat room, they samples were left in the buckets at room temperature for 14 days as a method of curing. This was done to allow the samples to slightly cure before the silane-based sealer was applied. The samples were then taken out of the buckets, and the silane-based sealer was coated on the exterior of the samples two times over two days. After the second application, the samples were left to dry for 7 days before being placed back into the buckets. The buckets were then placed into the controlled heat room at $38^{\circ} \mathrm{C}$ to continue the normal testing procedures. The samples of prisms, cylinders, and cores that were not coated with the silane-based sealer but are being compared to the samples that had the sealer applied underwent the same procedures as that of the samples with the sealer, minus the 
sealer application. All samples were measured as per CSA A23.2-14A (2009) at weeks 1, 2, 4, 8, 13, 18, 26, and 52 (and every three months until week 104 for samples containing SCM).

\subsection{Materials}

\subsubsection{Aggregate}

There were two coarse aggregates and one fine aggregate used in testing that will be discussed in this section. The two coarse aggregates consisted of virgin Sudbury aggregate and Sudbury RCA. The virgin Sudbury aggregate was obtained from a quarry in Sudbury, Ontario. Sudbury aggregate is a coarse gravel aggregate containing argillite, greywacke, and quartz-wacke. It is known to be a moderately reactive aggregate. Sudbury aggregate has a dry bulk relative density (BRD) of 2674 $\mathrm{kg} / \mathrm{m}^{3}$ and an absorption of $0.539 \%$. The Sudbury RCA was taken from three sources and are classified as RCA-H (obtained from high deteriorated road barriers), RCA-L (obtained from low deteriorated road barriers), and RCA-M (obtained from a mix of both high and low deteriorated road barriers). All the RCA was taken from a demolished bridge in Sudbury, Ontario built over 20 years ago. The BRD of the Sudbury RCA is $2359 \mathrm{~kg} / \mathrm{m}^{3}$ and an absorption is $3.873 \%$. A picture of high deteriorated core cast with Sudbury aggregate is shown in Figure 3.2.1. The core shows gel formation due to ASR surrounding the exposed aggregate. In some aggregates, the gel formation occurs within the aggregate as well as around the aggregate, however in Sudbury aggregate, the ASR gel formation is predominately around the aggregate. 


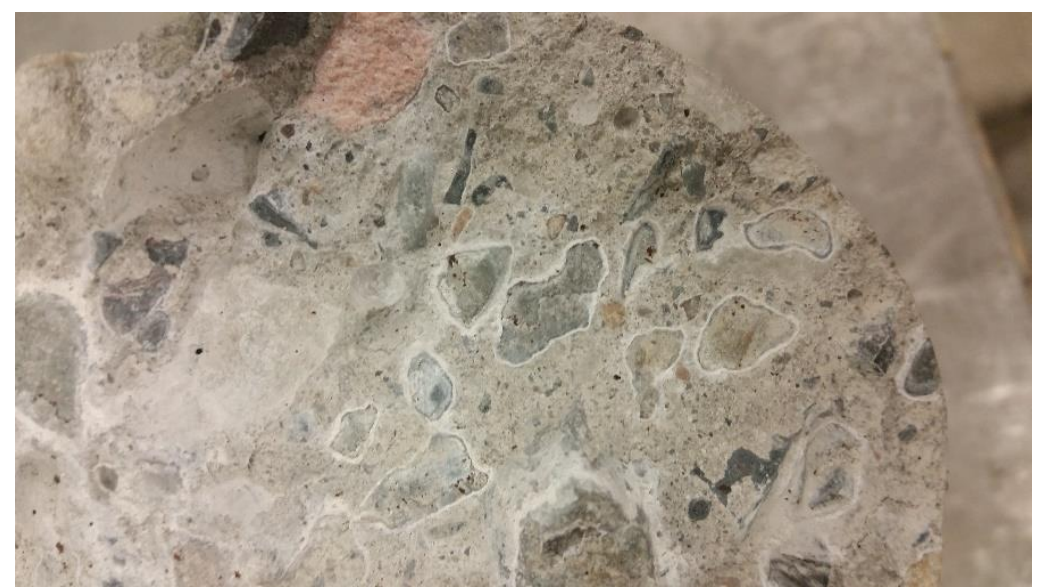

Figure 3.2.1: Gel formed from ASR exposed around aggregates at the surface of a concrete core taken from a bridge panel in Sudbury

The fine aggregate used is a non-reactive sand obtained from the Lafarge Caledon pit in Caledon, Ontario. The sand has a BRD of $2693 \mathrm{~kg} / \mathrm{m}^{3}$ with an absorption of $1.01 \%$. Table 3.2.1 shows a summary of the aggregate properties. The coarse aggregate testing was done in accordance with ASTM C127 - 12 Standard Test Method for Density, Relative Density (Specific Gravity), and Absorption of Coarse Aggregate (ASTM C127, 2015). The fine aggregate testing was done in accordance to ASTM C128 - 12 Standard Test Method for Density, Relative Density (Specific Gravity), and Absorption of Fine Aggregate (ASTM C128, 2015).

Table 3.2.1: Aggregate Properties

\begin{tabular}{|c|c|c|}
\hline & BRD (kg/m3) & Absorption (\%) \\
\hline $\begin{array}{c}\text { Virgin Sudbury } \\
\text { Aggregate }\end{array}$ & 2674 & 0.539 \\
\hline Sudbury RCA & 2359 & 3.873 \\
\hline Sand & 2693 & 1.01 \\
\hline
\end{tabular}




\subsubsection{Cementing Materials}

\subsubsection{General Use (GU) Portland Cement}

The majority of samples were cast using GU Portland cement, produced in Mississauga at the Holcim plant. The GU used for this research contained $0.99 \%$ total alkalis. The chemical analysis can be seen in Table 3.2.2.

\subsubsection{High Silica Fume Cement (HSF)}

The samples that were not cast using GU Portland cement were cast with HSF cement. HSF cement is a blended cement containing $92 \% \mathrm{GU}$ and $8 \%$ silica fume with a total alkali content of $0.96 \%$. This was used instead of mixing GU and silica fume in the lab. The chemical analysis can be seen in Table 3.2.2.

\subsubsection{Fly Ash}

There were three types of fly ash used as supplementary cementing materials (SCM) in this research. The first two are a low-calcium fly ash, labeled FA LA-LC and FA HA-LC, and the third was a high-calcium fly ash, labeled FA-HC. The low-alkali, low-calcium fly ash (FA LA-LC) contained $3.67 \%$ calcium oxide and $1.86 \% \mathrm{Na}_{2} \mathrm{O}_{\mathrm{e}}$ and the high-alkali low-calcium fly ash contained $7.24 \%$ calcium oxide and $3.68 \% \mathrm{Na}_{2} \mathrm{O}_{\mathrm{e}}$, while the high-calcium fly ash contained $26.41 \%$ calcium oxide and $2.11 \% \mathrm{Na}_{2} \mathrm{O}_{\text {e. }}$. The low-calcium fly ash labelled FA LA-LC was used in the AMBT and CMBT while FA HA-LC was used in the CPT. The rest of the chemical analysis is shown in Table 3.2.2. 


\subsubsection{Slag}

Slag was the final type of SCM used in this research. The chemical analysis can be seen in Table 3.2.2.

Table 3.2.2: Chemical Analysis of Cementing Materials

\begin{tabular}{|lccccccc|} 
Material & $\begin{array}{c}\text { CaO } \\
(\mathbf{\%})\end{array}$ & $\begin{array}{c}\text { SiO2 } \\
(\mathbf{\%})\end{array}$ & $\begin{array}{c}\text { Al203 } \\
(\mathbf{\%})\end{array}$ & $\begin{array}{c}\text { Fe203(T) } \\
(\mathbf{\%})\end{array}$ & $\begin{array}{c}\text { MgO } \\
(\mathbf{\%})\end{array}$ & $\begin{array}{c}\text { SO3 } \\
(\mathbf{\%})\end{array}$ & $\begin{array}{c}\text { Total } \\
\text { Alkalis (\%) }\end{array}$ \\
\hline $\begin{array}{l}\text { GU Portland } \\
\text { Cement }\end{array}$ & 62.77 & 19.33 & 5.25 & 2.42 & 2.35 & 4.03 & 0.99 \\
\hline HSF Cement & 55.97 & 26.15 & 5.03 & 2.18 & 2.22 & 4.02 & 0.96 \\
\hline FA-HC & 26.41 & 34.01 & 18.35 & 6.32 & 6.09 & 1.39 & 2.11 \\
\hline FA LA-LC & 3.67 & 47.36 & 23.86 & 17.4 & 1.0 & 1.08 & 1.86 \\
\hline FA HA-LC & 7.24 & 60.67 & 17.09 & 4.92 & 2.46 & 0.61 & 3.68 \\
\hline Slag & 39.9 & 36.9 & 7.82 & 0.68 & 11.2 & 0.45 & 1.10 \\
\hline
\end{tabular}

\subsubsection{Solutions}

\subsubsection{Sodium Hydroxide}

A sodium hydroxide solution was used for testing microbars and mortar bars. As per their respective standards, they are required to be placed in the oven at $80{ }^{\circ} \mathrm{C}$ in a solution of 1 mole of sodium hydroxide $(\mathrm{NaOH})$. The combination of a high temperature and exposure to a highly alkali solution (sodium hydroxide) causes an accelerated reaction of ASR.

\subsubsection{Sealers}

\subsubsection{Silane-based Sealer}

The silane-based sealer used in this research is known as MasterProtect $\mathrm{H} 1000$ and was manufactured by BASF. MasterProtect H 1000 is a clear liquid, containing 100\% silane sealer, and is used to protect new and existing concrete surfaces. As per the manufacturer, it is expected to "penetrate the concrete deeply, sealing out water, chloride ions, and acids, preventing damage 
from freeze/thaw cycles." For the purpose of this research, it was used to test its ability in reducing expansion due to ASR by reducing water penetration in concrete samples.

\subsection{Methodologies}

For ease of explanation, the research completed for this thesis will be broken up into two tasks. Task 1 will include mortar bars, microbars, and prisms containing a variety of SCM and two types of coarse aggregate. The only variation between mix designs in task 1 is the level and type of SCM and the type of aggregate used in each set of samples. Task 2 will consist of prisms, cylinders, cores, and road barriers. This task does not include the use of SCM, thus only control mixes are compared in casted samples. In task 2, two different mix designs, 3 types of coarse aggregate, and a silane-based sealer is used. All mixes were conducted as per their respective standards for ASR testing.

\subsubsection{Task 1}

Task 1 consists of using virgin Sudbury aggregate and Sudbury RCA-M for 12 different mixes. The only difference between each of the 12 mixes is the level and type of SCM. The 12 mixes are as follows:
1. Control
2. $25 \%$ FA-LC
3. $30 \%$ FA-HC
4. $50 \%$ HA-HC
5. $30 \%$ Slag
6. $40 \%$ Slag
7. $50 \%$ Slag
8. $\mathrm{HSF}$
9. HSF with $25 \%$ FA-LC
10. HSF with $30 \%$ FA-HC
11. HSF with $20 \%$ FA-HC
12. HSF with $30 \%$ Slag 
Each of the 12 mixes were cast for mortar bars, microbars, and prisms - once with virgin Sudbury aggregate, and once with Sudbury RCA-M. Thus, 24 sets of samples were cast for each of the three test methods. Mixes 1-7 were cast with GU Portland cement while mixes 8-12 were cast with HSF. The concrete prisms were all cast with a coarse-fine aggregate ratio of 60:40, cementing materials content of $420 \mathrm{~kg} / \mathrm{m}^{3}$, water-cement ratio of 0.42 and $\mathrm{Na}_{2} \mathrm{O}_{\mathrm{e}}$ of $1.25 \%$, as per CSA A23.2-14A (2009). The microbars were cast with a cement-aggregate ratio of $1: 1$, water-cement ratio of 0.32 , and $\mathrm{Na}_{2} \mathrm{O}_{\mathrm{e}}$ of $1.5 \%$ (Sommers, Nixon, and Sims, 2005). The mortar bars were cast with a cementaggregate ratio of 0.44 and water-cement ratio of 0.50 as per CSA A23.2-25A (2009). The purposes of this task is to determine (1) the difference in expansion between virgin Sudbury aggregate and Sudbury RCA, (2) the effectiveness of SCM on Sudbury RCA, and (3) the ability of the concrete microbar test and accelerated mortar bar test to properly predict the expansion of virgin Sudbury and Sudbury RCA in the concrete prism test.

\subsubsection{Task 2}

Task 2 consists of using virgin Sudbury aggregate, Sudbury RCA-H and Sudbury RCA-L for 6 different mixes as well as cores and barriers of high and low deterioration. The 6 mixes, all cast with GU Portland cement, are as follows, with the only differences being the aggregate type and the cement content:

1. Virgin Sudbury aggregate with $420 \mathrm{~kg} / \mathrm{m}^{3}$ cement

2. Virgin Sudbury aggregate with $360 \mathrm{~kg} / \mathrm{m}^{3}$ cement

3. RCA-H with $420 \mathrm{~kg} / \mathrm{m}^{3}$ cement

4. RCA-H with $360 \mathrm{~kg} / \mathrm{m}^{3}$ cement

5. RCA-L with $420 \mathrm{~kg} / \mathrm{m}^{3}$ cement

6. RCA-L with $360 \mathrm{~kg} / \mathrm{m}^{3}$ cement

The mixes cast with $420 \mathrm{~kg} / \mathrm{m}^{3}$ cement were classified as "Standard Mix" and the mixes cast with $360 \mathrm{~kg} / \mathrm{m}^{3}$ cement were classified as "Bridge Mix". The two types of cement contents were used 
because it is believed that the original bridge was cast with $360 \mathrm{~kg} / \mathrm{m}^{3}$ and the standard for ASR testing calls for the use of $420 \mathrm{~kg} / \mathrm{m}^{3}$. The 6 different mixes were cast for 2 sets of concrete prisms and 2 sets of concrete cylinders. One set of the cylinders and one set of the prisms were coated with the silane-based sealer. Each mix contains its respective cement content, a 60:40 coarse-fine aggregate ratio, and a 0.45 water-cement ratio, as per CSA A23.2-14A (2009). In addition to the 6 mixes, 2 sets of high deteriorated cores and 2 sets of low deteriorated cores ( 1 set each coated with the silane-based sealer) as well as 2 high deteriorated road barriers and 2 low deteriorated road barriers ( 1 of each coated with the silane-based sealer) belong to task 2 . The cores, cylinders, and prisms have all undergone the ASR testing procedures for concrete prisms while the barriers are undergoing field testing. The main purpose of this task is to be able to relate the expansion obtained on a standard prism $\left(420 \mathrm{~kg} / \mathrm{m}^{3}\right)$ undergoing ASR testing in the lab to the expansion in a road barrier in the field in effort to try and predict expansion due to ASR on structures. To do so, the following needs to be determined: (1) the effects of the level of cementing materials, (2) the effects of sample geometry, (3) the difference in expansion between extracted core samples and samples containing RCA from the same source as the core, (4) the difference in expansion between a core sample undergoing lab testing and its original source. In addition to the main objective, the use of a silane-based sealer is also being tested to determine its efficacy of mitigating or reducing expansion due to ASR of new and existing structures.

\subsubsection{Correlating Tasks 1 and 2}

Tasks 1 and 2 are designed to investigate different parameters. Task 1 is aimed at comparing results from the AMBT, CMBT, and CPT as well as determining the effectiveness of SCM on RCA. Task 2 is aimed at comparing data from different samples geometries and varying cement contents in order to better understand expansion occurring in structures. However, there are some correlations 
to be made between the two tasks. The first correlation to be made between task 1 and 2 is the difference in expansion between RCA-M (task 1), RCA-H (task 2), and RCA-L (task 2). Since RCA-M was received not knowing which barriers were crushed on the bridge in Sudbury to obtain it, the level of deterioration that had occurred to the RCA was not known. On the other hand, RCA-H and RCA-L was obtained by crushing known high and low deteriorated road barriers, thus it is no secret as to where those aggregates came from. The next correlation to be made between tasks 1 and 2 is the difference in effectiveness of mitigating ASR through SCM and the silanebased sealer. It is expected that the use of SCM will be more effective in reducing expansion, given the right amount and blends are used. That being said, it will be interesting to determine the level of reduction in expansion when silane-based sealer is used. 


\section{Chapter 4: Results and Analysis}

\subsection{Task 1}

Task 1 covers the testing of mortar bars, microbars, and prisms in an effort to determine the required SCM amounts and blends to effectively mitigate expansion due to ASR for Sudbury RCA. The results of each individual test will be evaluated and checked for consistency across all three test methods. The AMBT and CMBT will be evaluated for their effectiveness of properly predicting expansion results of Sudbury RCA for the CPT.

\subsubsection{Accelerated Mortar Bar Test}

The accelerated mortar bar test (AMBT) was conducted for 12 mixes containing virgin Sudbury aggregate and the same 12 mixes containing Sudbury RCA-M. Each mix consisted of different types, amounts, and blends of SCM with measurements taken for up to 28 days. An aggregate is generally deemed non-reactive if the expansion of the samples is less than $0.15 \%$ at 14 days (CSA A23.2-27A, 2009). If expansion results are between $0.10 \%$ and $0.20 \%$ at 14 days, the aggregate should be further tested (through CPT) and if the expansion results are greater than $0.20 \%$ the aggregate is considered to be ASR-reactive (ASTM C1260, 2014). An aggregate with its control mix expanding between $0.15 \%$ and $0.40 \%$ is considered highly reactive and an aggregate with its control mix expanding over $0.40 \%$ is considered extremely reactive (CSA A23.2-27A, 2009).

\subsubsection{AMBT Results for Virgin Sudbury Aggregate}

The first samples tested using the AMBT were the samples cast with virgin Sudbury aggregate containing GU Portland cement. It is no surprise that the control mix, which contains no SCM, far exceeded the allowable limit of $0.15 \%$ at 14 days. With the introduction of SCM, the expansion values begin to decrease. Beginning with fly ash, both low-calcium (FA LA-LC) and high-calcium 
fly ash (FA-HC) were introduced to the aggregate. Samples containing 25\% FA LA-LC, 30\% FA$\mathrm{HC}$, and 50\% FA-HC were evaluated and compared to the expansion results of the control mix. These results can be seen in Figure 4.1.1, in which the control mix shows the most expansion, $0.26 \%$ at 14 days (considered to be highly reactive) and $0.46 \%$ at 28 days, and the $25 \%$ FA LALC shows the least expansion, $0.03 \%$ at 14 days and $0.09 \%$ at 28 days. The mixes containing FA$\mathrm{HC}$ fall between the two, with the samples containing 50\% FA-HC expanding $0.10 \%$ at 14 days and $0.18 \%$ at 28 days and the samples containing $30 \%$ FA-HC expanding $0.23 \%$ at 14 days and $0.38 \%$ at 28 days.

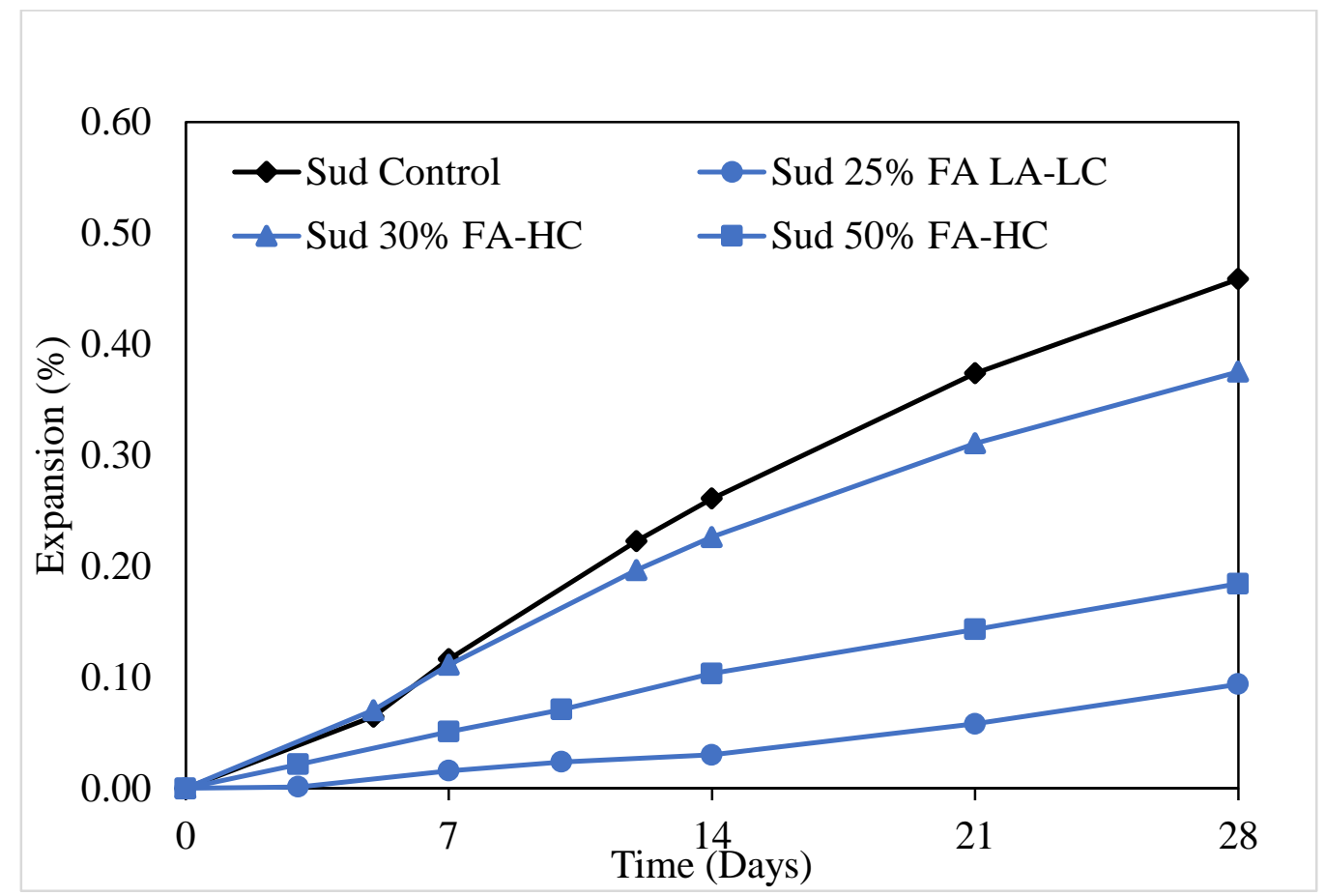

Figure 4.1.1: AMBT Expansion results of Sudbury control and Sudbury containing fly ash

Along with the virgin Sudbury control mix, the virgin Sudbury containing 30\% FA-HC mix also far exceeds their $0.15 \%$ expansion limit at 14 days. The only safe mixes are the virgin Sudbury containing 25\% FA LA-LC and virgin Sudbury with 50\% FA-HC, as they are significantly less than the limit. 
In addition to the testing of fly ash, slag was introduced to virgin Sudbury aggregate in $30 \%, 40 \%$, and $50 \%$ cementations material replacements. As expected, as the amount of slag increases, the level of expansion decreases, shown in Figure 4.1.2. When 30\% slag is introduced, the expansion reduces to $0.18 \%$ at 14 days and $0.34 \%$ at 28 days. With $40 \%$ slag, the expansion reduces to $0.11 \%$ at 14 days and $0.22 \%$ at 28 days. Finally, when $50 \%$ slag is introduced, the 14 day expansion reduces to $0.04 \%$ and the 28 day expansion reduces to $0.10 \%$.

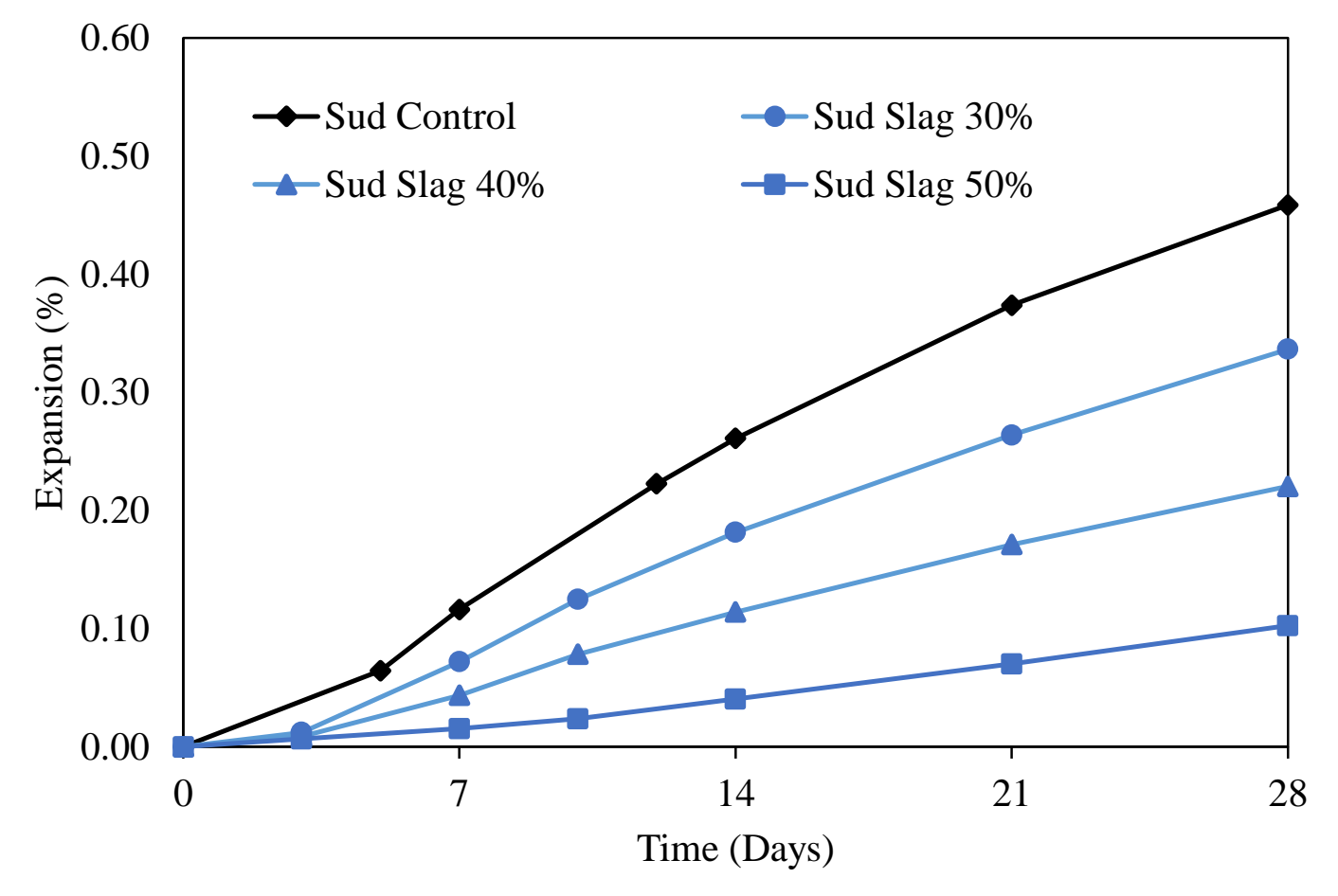

\section{Figure 4.1.2: AMBT Expansion results of Sudbury control and Sudbury containing slag}

The results of the AMBT test for the samples containing slag suggest that $30 \%$ slag is not sufficient to mitigate ASR in virgin Sudbury aggregate and should be further tested using the CPT, as its expansion value falls between $0.10 \%$ and $0.20 \%$. When $40 \%$ slag and $50 \%$ slag is introduced, the expansion of $0.11 \%$ and $0.04 \%$ at 14 days, respectively, suggests that virgin Sudbury aggregate is safe to use with $40 \%$ or $50 \%$ slag replacement. 
The next portion of tested samples consisted of replacing the GU Portland cement with high silica fume (HSF) blended cement, which contains $8 \%$ silica fume and $92 \%$ GU Portland cement. When introducing the HSF cement, the expansion reduces significantly in comparison to the Sudbury control mix containing GU Portland cement, expanding $0.08 \%$ at 14 days and $0.24 \%$ at 28 days. In addition, when introducing fly ash and slag to the mixes containing HSF cement, the expansion continued to reduce. Three variations of fly ash and one mix containing slag were used with HSF - 25\% FA LA-LC, 30\% FA-HC, 20\% FA-HC, and 30\% slag. The results can be seen in Figure 4.1.3, with the mix containing HSF with $25 \%$ FA LA-LC produced the least expansion, expanding $0.02 \%$ at 14 days and $0.04 \%$ at 28 days, followed by the mix containing HSF with $30 \%$ slag, expanding $0.03 \%$ at 14 days and $0.05 \%$ at 28 days. The mixes containing HSF with $30 \%$ FAHC and HSF with 20\% FA-HC followed, expanding $0.03 \%$ at 14 days, $0.10 \%$ at 28 days and $0.05 \%$ at 14 days, $0.14 \%$ at 28 days, respectively.

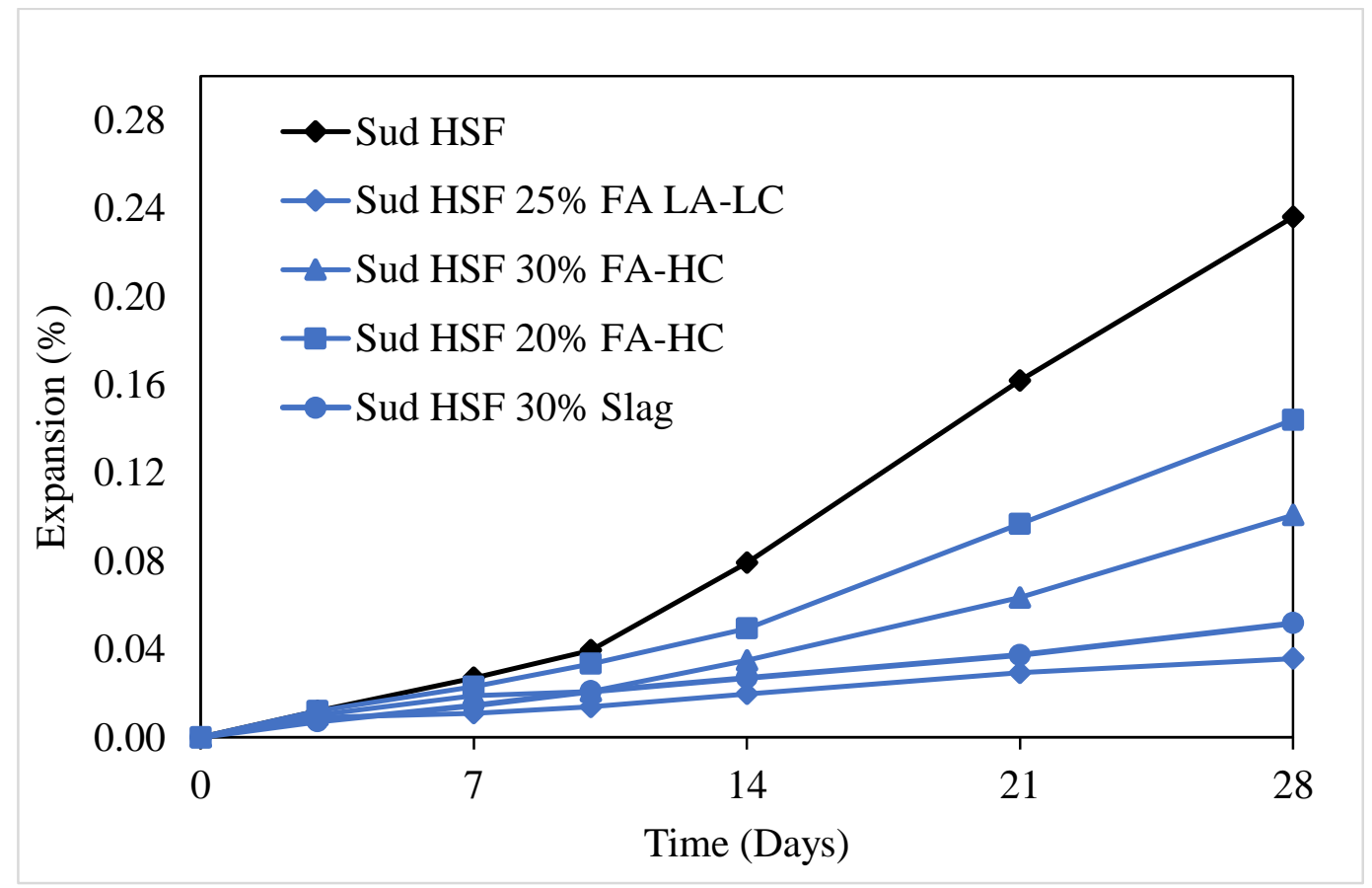

Figure 4.1.3: AMBT Expansion results of Sudbury containing HSF and various SCM 
Figure 4.1.3 shows that by replacing GU Portland cement with HSF blended cement, the AMBT suggests that virgin Sudbury aggregate can be used, as the expansion is below $0.15 \%$ at 14 days in all tested samples. The mixes containing either fly ash or slag in addition to HSF blended cement yield expansion results under $0.05 \%$, thus are deemed safe to use by the AMBT.

As shown in Figures 4.1.1, 4.1.2, and 4.1.3 there does not seem to be any outliers or strange changes to any of the curves, as the trend of the expansion results of all 12 mixes over 28 days seems to be smooth and accurate. When looking at the 14 day expansion of all the samples containing virgin Sudbury aggregate in Figure 4.1.4, 6 of the 12 mixes are significantly below the 0.15\% expansion limit (25\% FA LA-LC, 50\% slag, HSF with 25\% FA LA-LC, HSF with $30 \%$ FA-HC, HSF with $20 \%$ FA-HC, and HSF with $30 \%$ slag). Three of the mixes (50\% FA-HC, $40 \%$ slag, and HSF) are very close to the limit and the $30 \%$ slag mix is between the $0.15 \%$ and $0.20 \%$ limits, thus require further testing. It can be assumed that the final two mixes (Sudbury control and $30 \%$ FA-HC) are ASR reactive, as they exceed $0.20 \%$ expansion. It should be noted that the most effective SCM in reducing expansion of virgin Sudbury aggregate in the AMBT is the low-calcium fly ash, followed by the slag, leaving the high-calcium fly ash being the least effective. When used in combination with HSF, the effects are greater. 


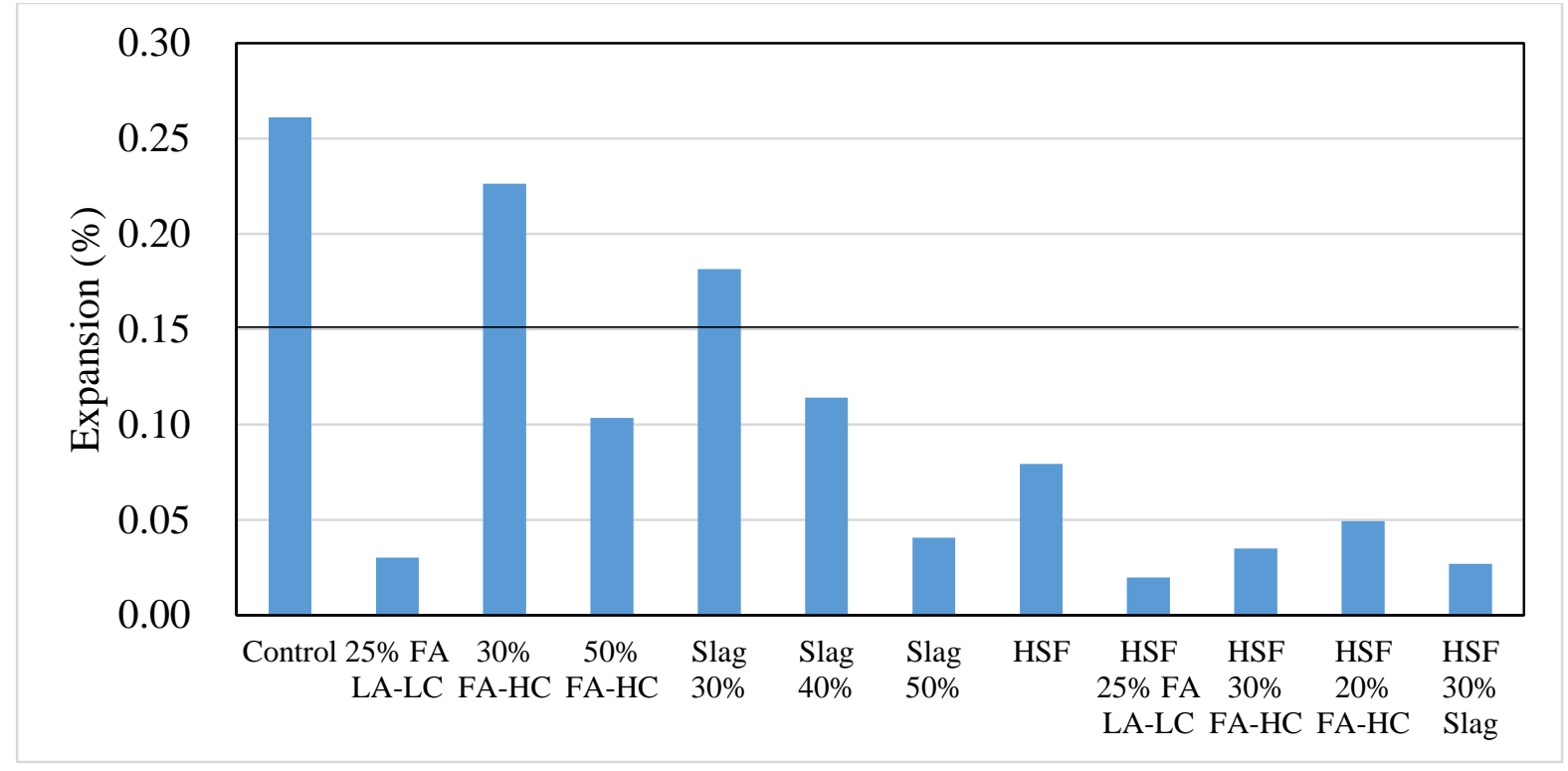

Figure 4.1.4: AMBT summary of 14 day expansion results for virgin Sudbury aggregate

\subsubsection{AMBT Results for Sudbury RCA-M}

When testing Sudbury RCA with the AMBT, similar trends prevailed. As expected, the RCA control mix with GU Portland cement contained the highest expansion results, expanding 0.19\% at 14 days and $0.31 \%$ at 28 days. When compared with 25\% FA LA-LC, 30\% FA-HC, and 50\% FA-HC, the expansion decreased similar to that of the samples containing virgin Sudbury aggregate, shown in Figure 4.1.5. Again, the samples containing 25\% FA LA-LC expanded the least, with $0.06 \%$ expansion at 14 days and $0.15 \%$ at 28 days, followed by $50 \%$ FA-HC, with $0.12 \%$ expansion at 14 days and $0.20 \%$ expansion at 28 days. Sudbury RCA containing $30 \%$ FA$\mathrm{HC}$ followed, showing expansion results of $0.17 \%$ at 14 days and $0.27 \%$ at 28 days. 


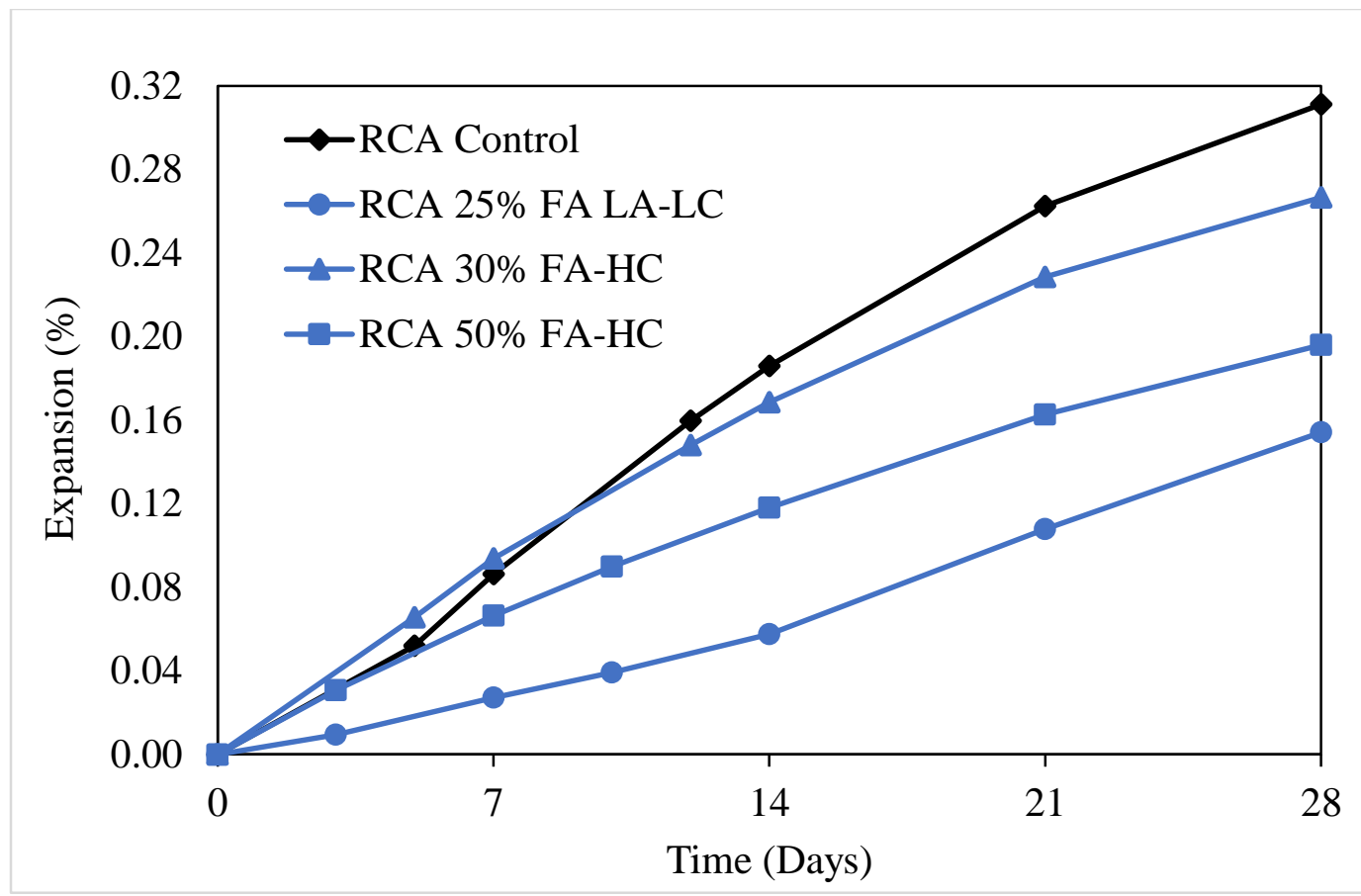

Figure 4.1.5: AMBT Expansion results of Sudbury RCA control and Sudbury RCA containing fly ash

Figure 4.1.5 shows that two samples were below the $0.15 \%$ limit at 14 days, Sudbury RCA with $25 \%$ FA LA-LC and 50\% FA-HC. The RCA control and RCA 30\% FA-HC both exceed the $0.15 \%$ limit, but are below the $0.20 \%$ limit, suggesting further testing is required through the CPT. Judging by the curve shown in Figure 4.1.5, the expansion in the RCA control mix seems to start off slow over the first 7 days, thus the actual expansion in that mix may be slightly higher than the test revealed.

When introducing slag as the SCM to Sudbury RCA aggregate, similar trends occurred as that of the virgin Sudbury aggregate. With 30\%, 40\%, and 50\% slag replacements, expansion again decreased as the percentage of slag increased, shown in Figure 4.1.6. The RCA samples containing $30 \%$ slag expanded $0.12 \%$ at 14 days and $0.22 \%$ at 28 days, while RCA with $40 \%$ slag expanded $0.09 \%$ at 14 days and $0.19 \%$ at 28 days. The largest reduction in expansion with the use of slag 
occurred with $50 \%$ slag replacement, yielding an expansion of $0.06 \%$ at 14 days and $0.13 \%$ at 28 days.

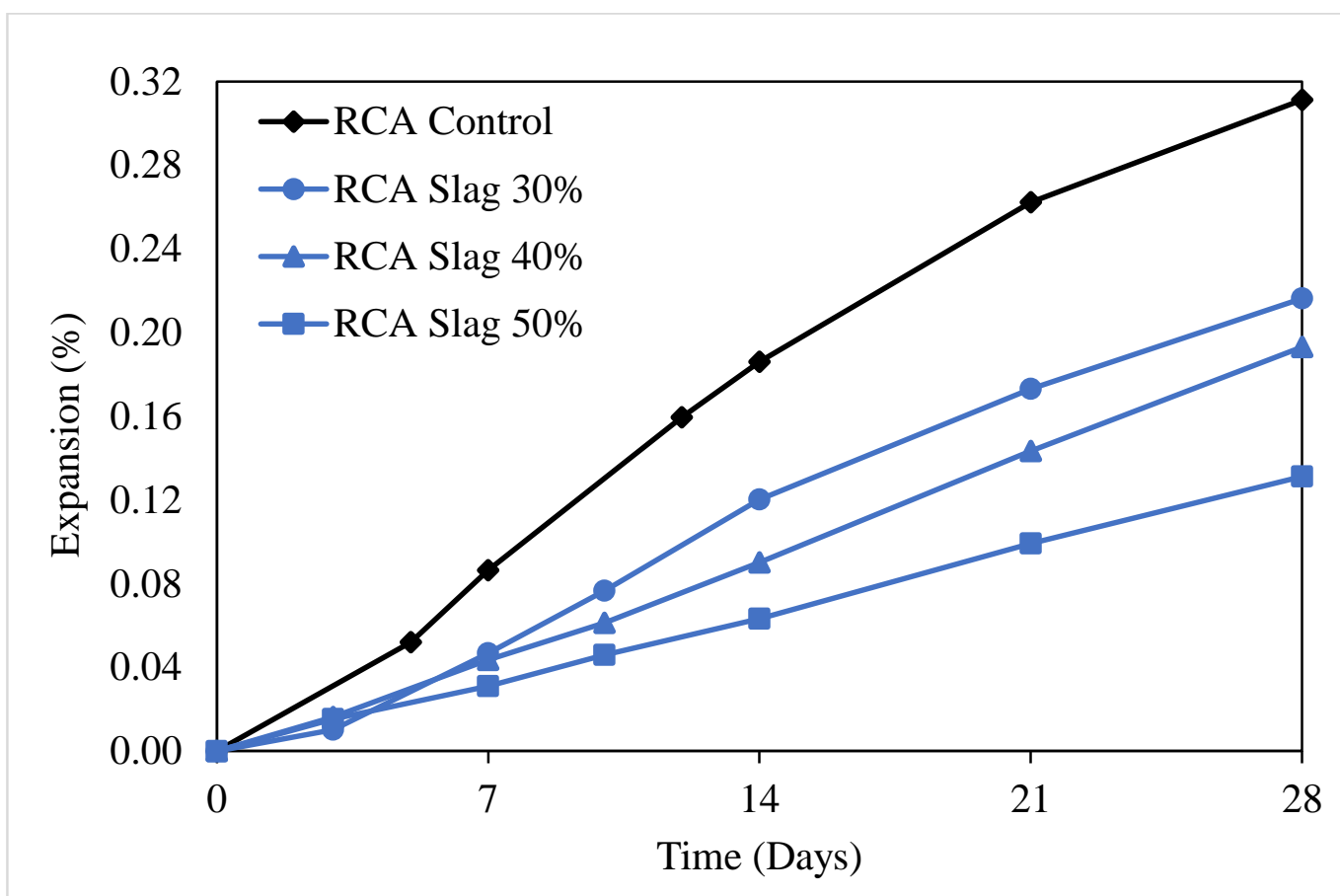

\section{Figure 4.1.6: AMBT Expansion results of Sudbury RCA control and Sudbury RCA containing slag}

The results of the AMBT for Sudbury RCA containing various portion of slag show that when introducing $40 \%$ or $50 \%$ slag, Sudbury RCA expands well below the $0.15 \%$ limit, thus are acceptable for use. With a $30 \%$ replacement, the $0.12 \%$ expansion at 14 days is very close to the limit, thus requires further testing through the CPT.

When replacing GU Portland cement with HSF blended cement in samples cast with Sudbury RCA, the expansion reduced to $0.08 \%$ at 14 days and $0.22 \%$ at 28 days. The expansion results continued to decrease with the addition of 25\% FA LA-LC, 30\% FA-HC, 20\% FA-HC, and 30\% slag, shown in Figure 4.1.7. The least expansion was experienced in the samples containing HSF with $25 \%$ FA LA-LC, showing expansion of $0.04 \%$ at 14 days and $0.08 \%$ at 28 days, followed 
by HSF with $30 \%$ slag, showing expansion of $0.05 \%$ at 14 days and $0.11 \%$ at 28 days. The HSF with $30 \%$ FA-HC followed, expanding $0.08 \%$ at 14 days and $0.17 \%$ at 28 days, with the HSF with $20 \%$ FA-HC expanding slightly more at $0.08 \%$ at 14 days and $0.19 \%$ at 28 days.

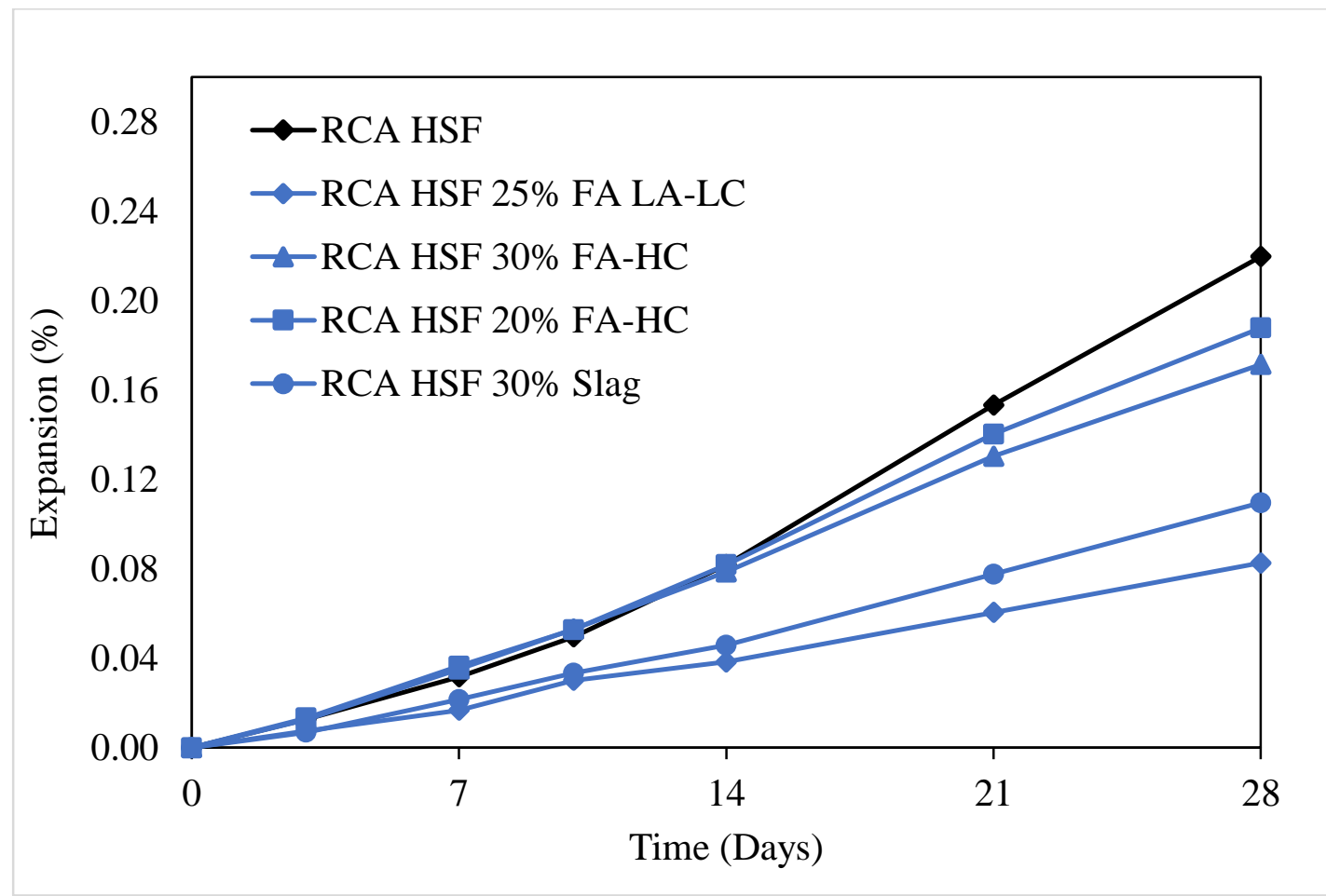

Figure 4.1.7: AMBT Expansion results of Sudbury RCA containing HSF and various SCM

The results of the AMBT in Figure 4.1.7 show that all Sudbury RCA samples containing HSF blended cement fall below the $0.15 \%$ expansion limit at 14 days. This suggests that with the use of HSF blended cement, Sudbury RCA can be used.

In the case of the Sudbury RCA-M mortar bars, shown in Figure 4.1.8, all mixes but the control mix, as explained previously, follow a steady trend and don't seem to have any strange changes to the curve or outliers. When looking at the 14 day expansion of all the samples containing Sudbury RCA, 10 of 12 mixes (RCA with 25\% FA LA-LC, RCA with 50\% FA-HC, RCA with 30\%, 40\%, and 50\% slag, RCA with HSF, RCA with HSF and 25\% FA LA-LC, RCA with HSF and 30\% FA-HC, RCA with HSF and 20\% FA-HC, and RCA with HSF and 30\% slag) 
are deemed safe for use, as they are well below the $0.15 \%$ expansion limit. The remaining 2 mixes (RCA control and RCA with 30\% FA-HC) are between the $0.15 \%$ and $0.20 \%$ limits, thus require further testing. The order of effectiveness of the SCM in reducing expansion of Sudbury RCA-M in the AMBT is consistent with that of the virgin aggregate. The low-calcium fly ash is the most effective, followed by the slag, with the high-calcium fly ash being the least effective. When used in combination with HSF, the effects are greater.

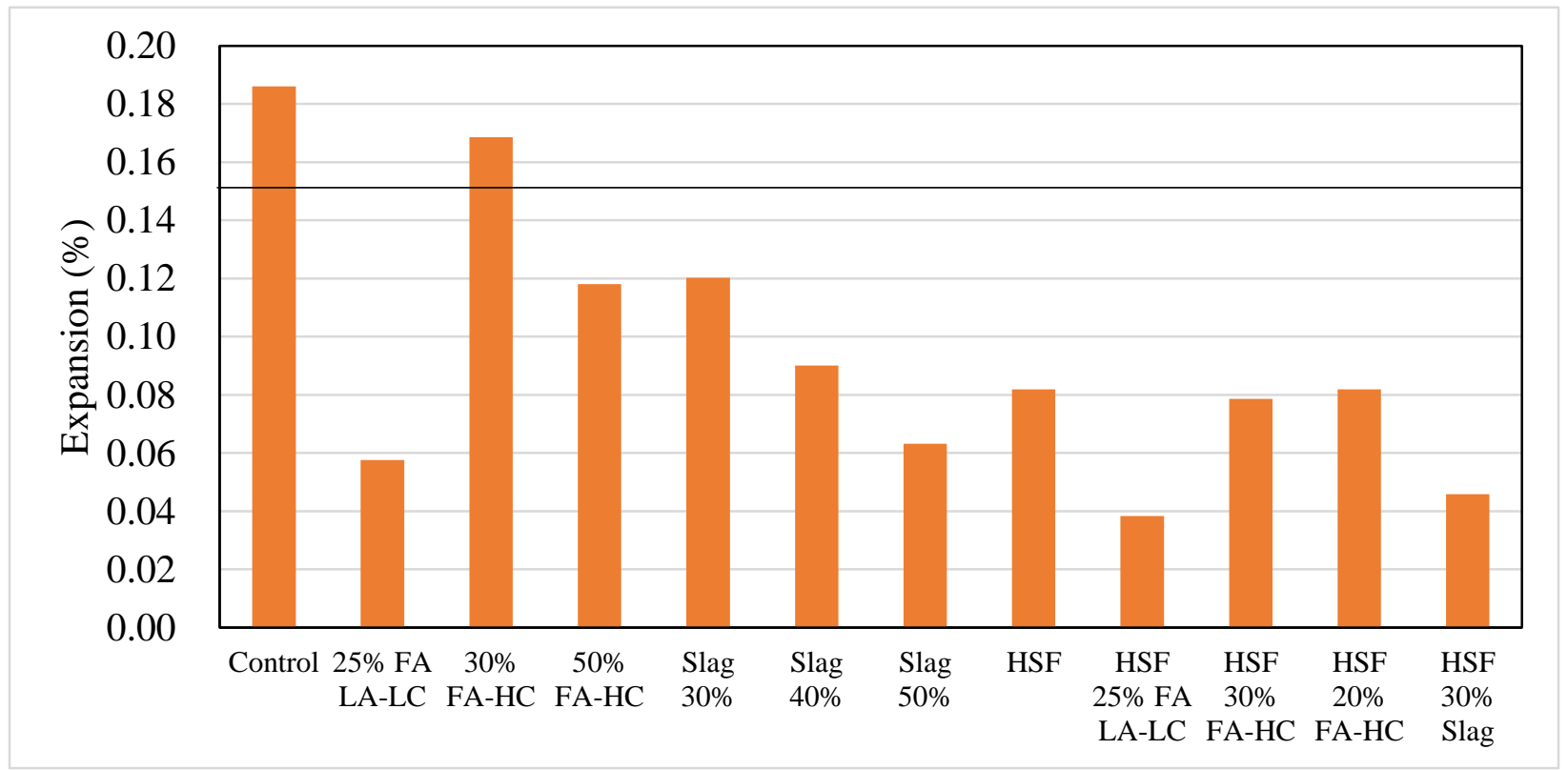

Figure 4.1.8: AMBT summary of 14 day expansion results for Sudbury RCA-M

\subsubsection{Comparing AMBT Results of Virgin Sudbury Aggregate and Sudbury RCA-M}

When comparing the results of the AMBT between samples cast with virgin Sudbury aggregate and Sudbury RCA-M, it is difficult to determine which aggregate expands at a higher rate. In some cases, the virgin Sudbury aggregate does, while in other cases the Sudbury RCA-M does. In Figure 4.1.9, the 14-day mortar bar expansion data can be seen for both aggregates containing all variations of SCM used. 


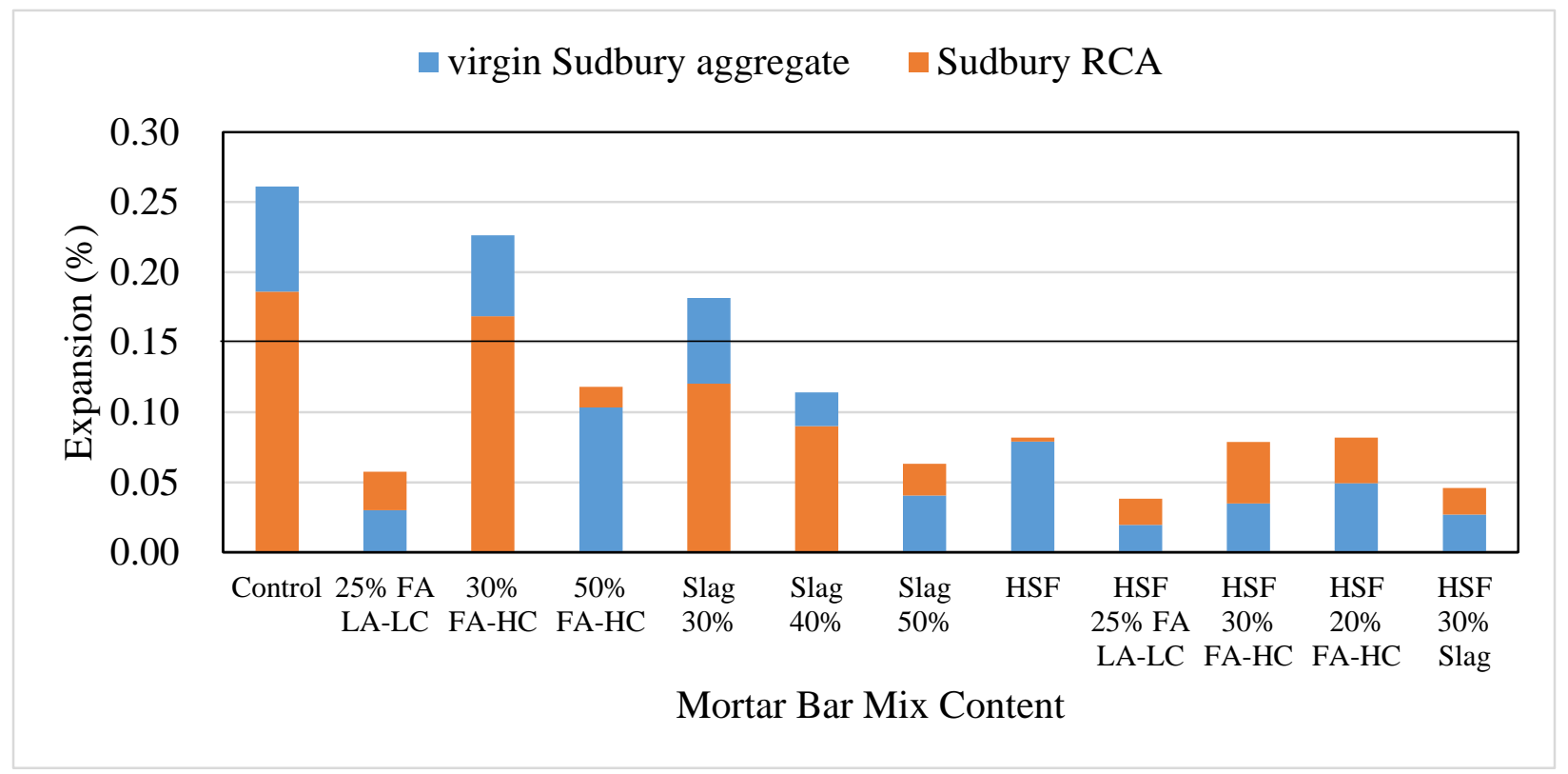

Figure 4.1.9: AMBT Summary of 14-day expansion results

As shown in Figure 4.1.9, the expansion of the same mix containing virgin aggregate exceeds the expansion of the RCA-M 4 out of 12 times, especially in the case of the control mixes and the mixes containing 30\% FA-HC. In the other two cases, the mixes containing $30 \%$ and $40 \%$ slag, the difference in expansion between the two is minimal. This trend was monitored up until the 28day mark to see if these 4 mixes continued to expand at a higher rate with the virgin aggregate. The 28-day expansion results can be seen in Figure 4.1.10. 


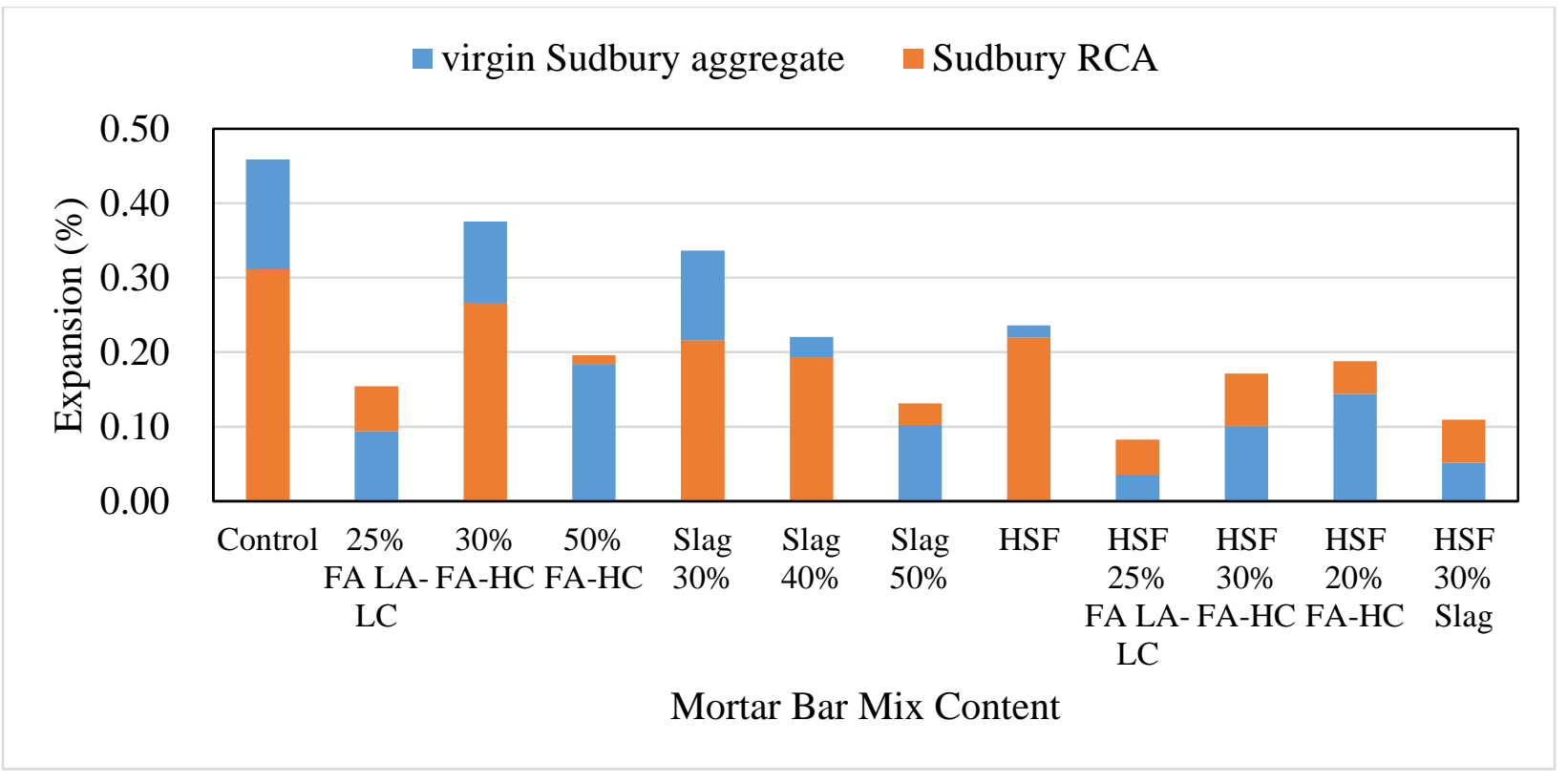

Figure 4.1.10: AMBT Summary of 28-day expansion results

When looking at the 28-day expansion in Figure 4.1.10, the same trend occurs, along with the mix containing HSF cement and virgin Sudbury aggregate slightly exceeding the HSF cement and Sudbury RCA-M expansion. In order to verify the results, the control mixes and the mixes containing 30\% FA-HC were repeated. Figure 4.1.11 shows that after the repeat test, the same trend occurs at 28 days.

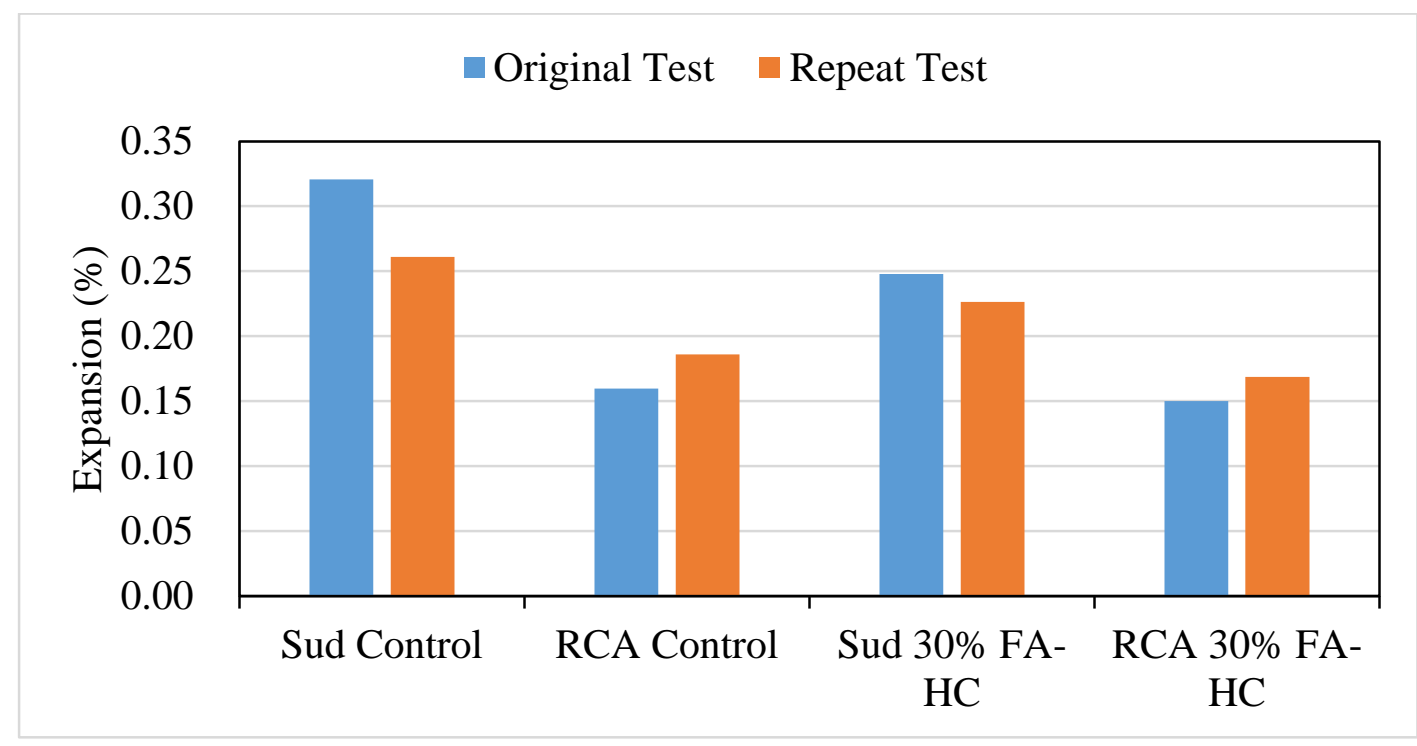

Figure 4.1.11: 28-day repeat results of AMBT 
The data obtained from the repeat tests shows that both the control mix and the mix containing $30 \%$ FA-HC using virgin Sudbury aggregate do in fact expand at a higher rate than that of the same mixes using Sudbury RCA-M. The second time around, the difference seems to be slightly less apparent, which is likely more realistic as it is closer to the trend of the rest of the mixes. Through the use of the AMBT, it is unknown whether the virgin Sudbury aggregate or the Sudbury RCA-M produce higher expansion results. These results will be compared to the CMBT and the CPT in order to further verify the results obtained. A summary of the 14-day expansion results are shown in Table 4.1.1.

Table 4.1.1: Summary of 14-day expansion data for AMBT

\begin{tabular}{|c|c|c|}
\hline \multirow{2}{*}{ Mix Type } & \multicolumn{2}{|c|}{ 14-day expansion value (\%) } \\
\cline { 2 - 3 } & $\begin{array}{c}\text { Virgin Sudbury } \\
\text { Aggregate }\end{array}$ & Sudbury RCA-M \\
\hline Control & 0.261 & 0.186 \\
\hline $25 \%$ FA LA-LC & 0.030 & 0.058 \\
\hline $30 \%$ FA-HC & 0.226 & 0.169 \\
\hline $50 \%$ FA-HC & 0.103 & 0.118 \\
\hline $30 \%$ Slag & 0.182 & 0.120 \\
\hline $40 \%$ Slag & 0.114 & 0.090 \\
\hline $50 \%$ Slag & 0.041 & 0.063 \\
\hline HSF (8\% Silica Fume) & 0.079 & 0.082 \\
\hline HSF w/ 25\% FA LA-LC & 0.020 & 0.038 \\
\hline HSF w/ 30\% FA-HC & 0.035 & 0.079 \\
\hline HSF w/ 20\% FA-HC & 0.049 & 0.082 \\
\hline HSF w/ 30\% Slag & 0.027 & 0.046 \\
\hline
\end{tabular}




\subsubsection{Concrete Microbar Test}

The concrete microbar test (CMBT) was conducted for the same 12 mixes containing virgin Sudbury aggregate and Sudbury RCA-M as the AMBT. An aggregate is suggested to be safe if the expansion of the samples is less than $0.10 \%$ for siliceous limestone aggregates and $0.04 \%$ for any other siliceous aggregates at 28 days (Grattan-Bellew et. al, 2004). Since Sudbury aggregate is a gravel containing Argillite, greywacke and quartz-wacke, the 28 -day limit is $0.04 \%$. Although the current expansion limit is set at 28 days, measurements were taken up to 56 days to further monitor the expansion. Sommers, Nixon, and Sims (2005) suggests using an aggregates between $4.75 \mathrm{~mm}$ and $9.5 \mathrm{~mm}$, however two sizes were checked $(4.75 \mathrm{~mm}-9.5 \mathrm{~mm}$ and $9.5 \mathrm{~mm}-12.5 \mathrm{~mm})$ with virgin Sudbury, Sudbury RCA-M, and virgin Spratt aggregate to determine which would show more reactivity. The results, shown in Figure 4.1.12, verify that the $4.75 \mathrm{~mm}-9.5 \mathrm{~mm}$ mixes expand at a higher rate, thus all mixes were conducted using aggregate of that size.

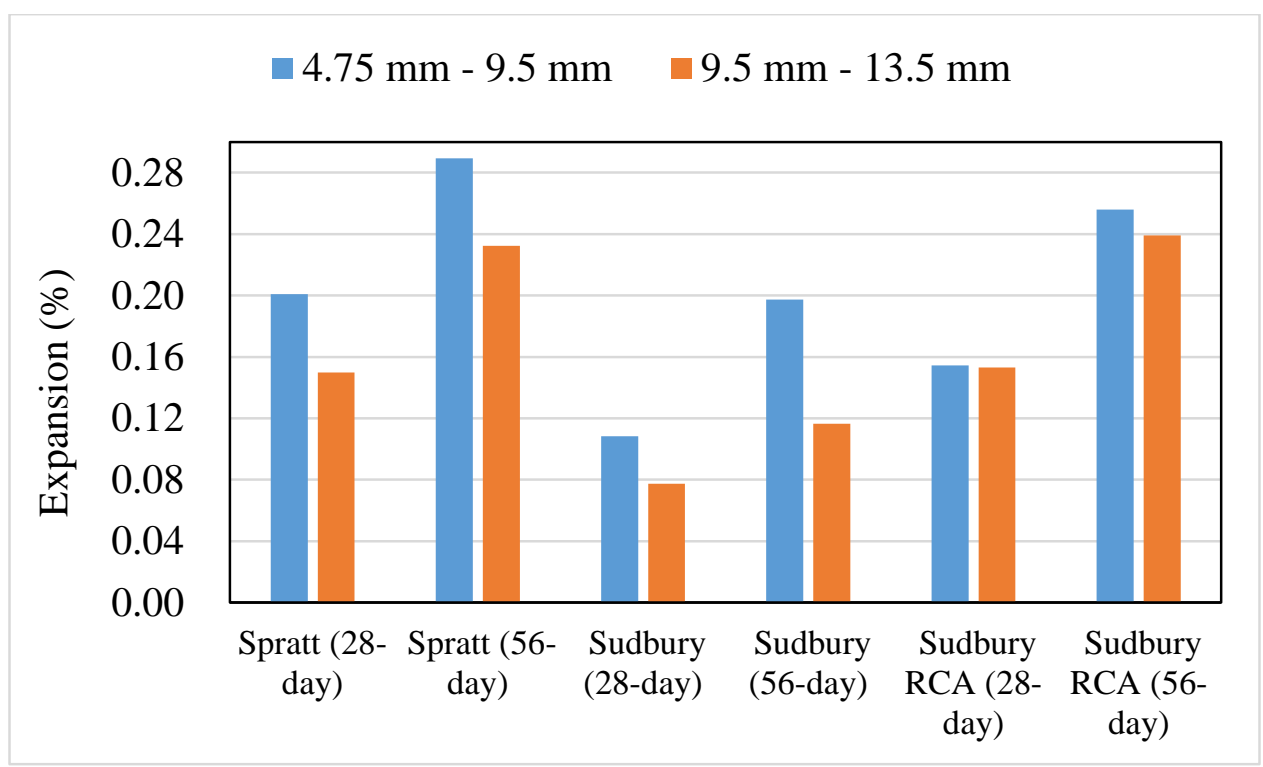

Figure 4.1.12: Spratt, Sudbury, and Sudbury RCA-M CMBT expansion results 


\subsubsection{CMBT Results for Virgin Sudbury Aggregate}

The CMBT results for virgin Sudbury aggregate are expected to follow a similar trend to that of the results of the AMBT. The first set of mixes will look at comparing the control mix of virgin Sudbury aggregate and GU Portland cement with mixes containing different types and levels of fly ash. As shown in Figure 4.1.13, the expansion of the CMBT for virgin Sudbury aggregate containing 30\% FA-HC exceeds the expansion of the virgin Sudbury aggregate with no SCM. This is should not be the case and it seems that the expansion of the microbars containing 30\% FA-HC should be slightly lower or the control mix should be slightly higher. The mix containing $30 \%$ FAHC seems to follow a steady curve, while the Sudbury control mix does not. The expansion over the first 21 days of the control mix seems to be rather slow, thus suggesting the actual expansion should be slightly higher. That being said, the 28-day expansion of the Sudbury control, Sudbury with 30\% FA-HC, and Sudbury with 50\% FA-HC are all above the $0.04 \%$ limit, with expansion values of $0.11 \%, 0.14 \%$, and $0.09 \%$ respectively. The 28 -day expansion of the Sudbury with $25 \%$ FA LA-LC is right on the acceptable limit of $0.04 \%$, thus further testing is required to verify its reactivity. 


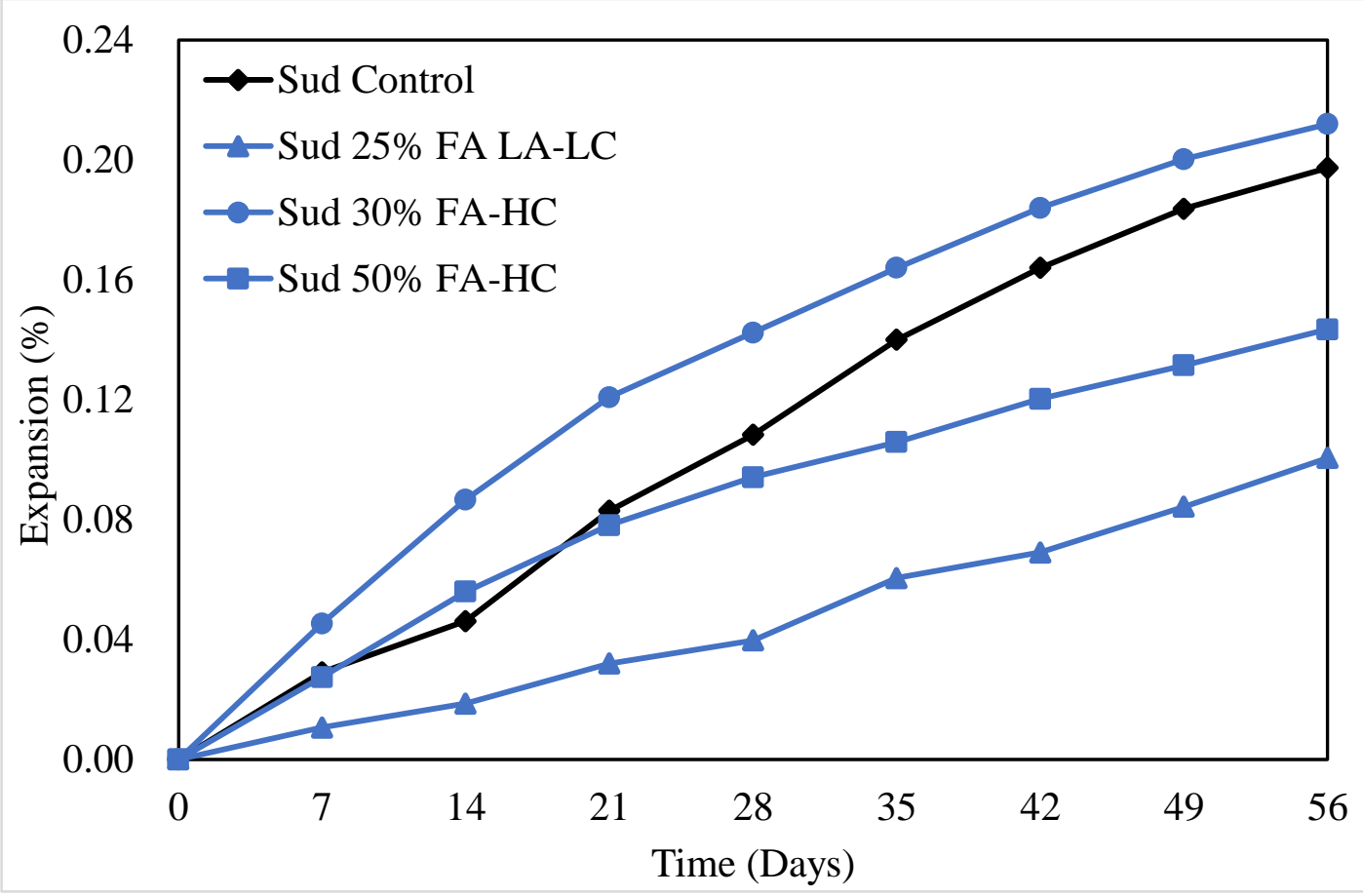

Figure 4.1.13: CMBT Expansion results of Sudbury control and Sudbury containing fly ash

Following fly ash, the next set of mixes to be compared are the Sudbury control mix and Sudbury containing various levels of slag. 30\%, 40\%, and 50\% slag mixed with GU Portland cement was used and expansion reduced as more slag was introduced. The mix containing $30 \%$ slag had a 28 day expansion of $0.08 \%$ and a 56 -day expansion of $0.16 \%$, the mix containing $40 \%$ slag had a 28 day expansion of $0.04 \%$ and a 56-day expansion of $0.08 \%$, while the mix containing $50 \%$ slag had a 28-day expansion of $0.03 \%$ and a 56-day expansion of $0.05 \%$, shown in Figure 4.1.14. As expected, all expansion results are below that of the Sudbury control mix, which has a 28-day expansion of $0.11 \%$ and a 56 -day expansion of $0.20 \%$. The mix containing $40 \%$ slag is right on the 28-day limit and the mix containing 50\% slag is slightly below the limit. 


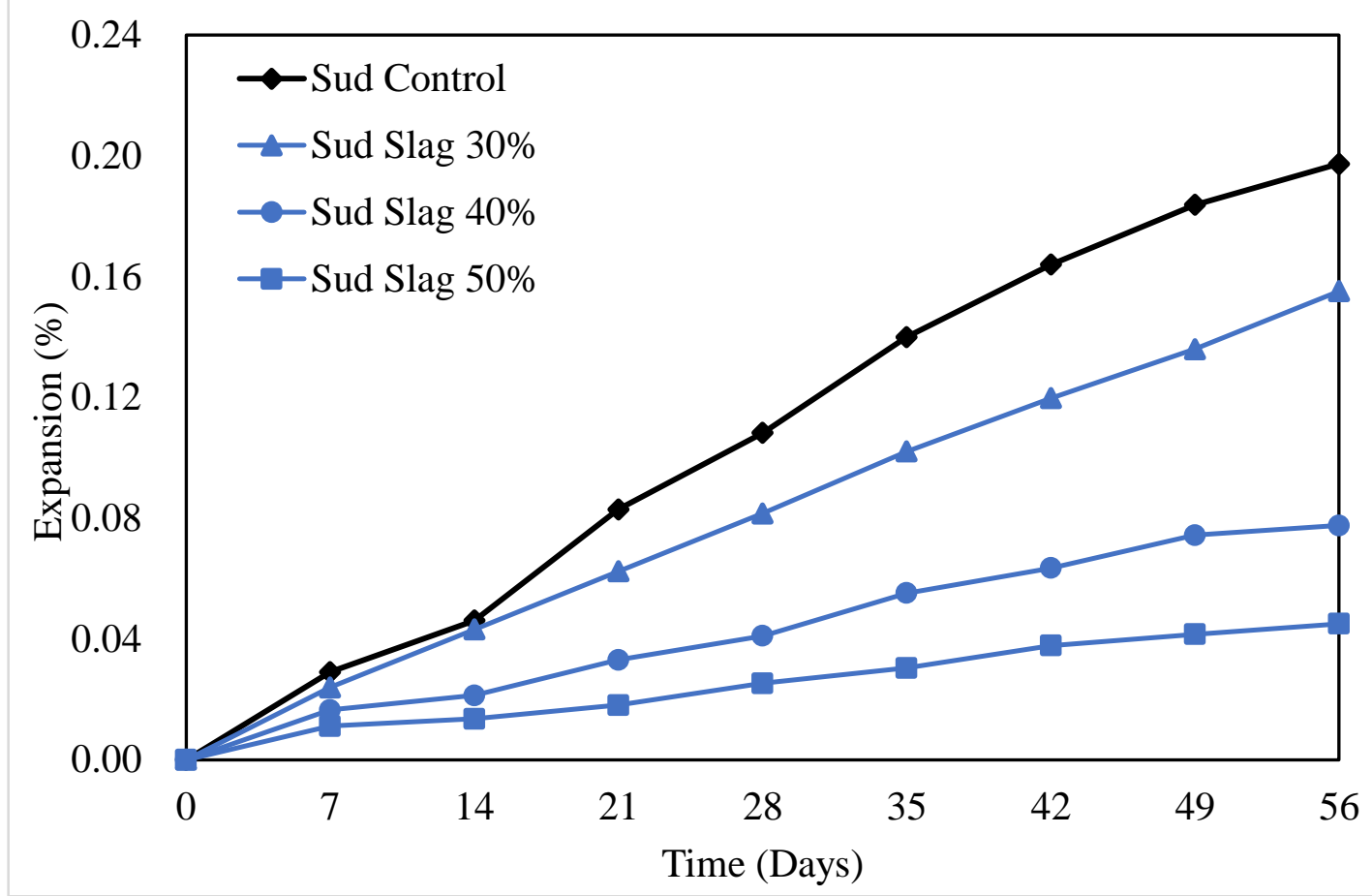

Figure 4.1.14: CMBT Expansion results of Sudbury control and Sudbury containing slag

In the next set of samples, GU Portland cement was replaced with HSF blended cement. The virgin Sudbury aggregate with HSF cement mix was compared to that of mixes containing virgin Sudbury aggregate with HSF and the addition of FA LA-LC, FA-HC, and slag. The expansion results for the mix containing HSF expanded just over $0.04 \%$ at 28 days and $0.10 \%$ at 56 days. The HSF with low-calcium, high-calcium fly ashes, and slag were, as expected, all below or on the $0.04 \%$ expansion limit at 28-days, with the mix containing HSF and 25\% FA LA-LC expanding the least (28-day expansion of $0.03 \%$ and 56-day expansion of just under $0.04 \%$ ), followed by the HSF with $30 \%$ slag ( $0.03 \%$ expansion at 28 days and $0.05 \%$ expansion at 56 days), followed by the mix containing HSF and 30\% FA-HC (28-day expansion of $0.04 \%$ and 56-day expansion of $0.07 \%$ ), and the mix containing HSF and 20\% FA-HC (28-day expansion of 0.04\% and 56-day expansion of $0.09 \%$ ). 


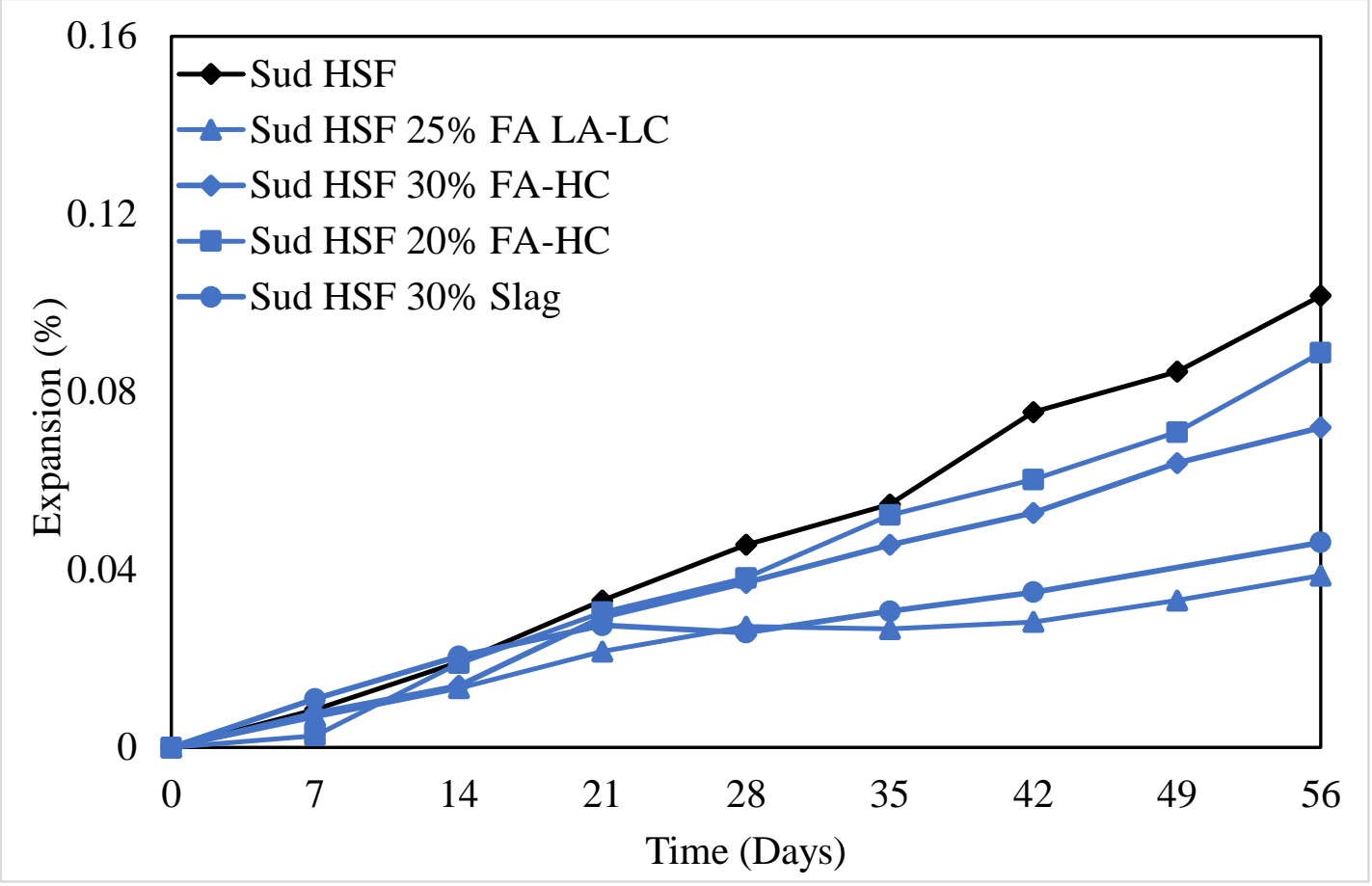

Figure 4.1.15: CMBT Expansion results of Sudbury containing HSF and various SCM

When looking at the 28-day CMBT expansion data of all mixes, shown in Figure 4.1.16, it is apparent that 7 of the 12 mixes are within the limit of $0.04 \%$ at 28 days. That being said, 2 of them (mixes containing 25\% FA LA-LC and 40\% slag) are right on the limit, 2 are just below the limit (mixes containing HSF with $30 \%$ FA-HC and HSF with $20 \%$ FA-HC), while the other three (mixes containing 50\% slag, HSF with 25\% FA LA-LC, and HSF with 30\% slag) are well below the limit. These mixes, along with the HSF, slightly above the $0.04 \%$ limit will be further tested through the CPT to determine their reactivity. The other 4 mixes contain expansion results far beyond the limit and can be classified as reactive by the CMBT. 


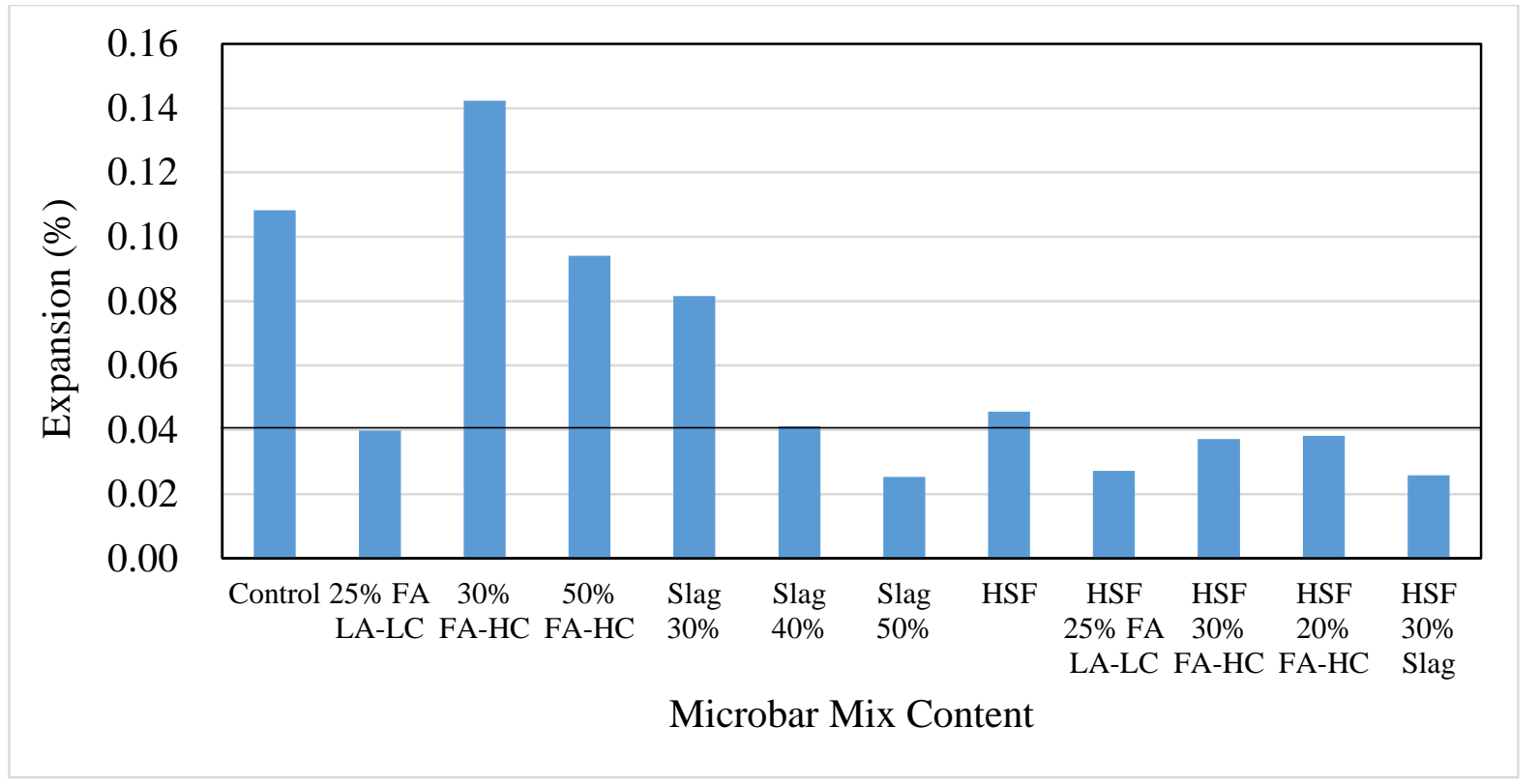

Figure 4.1.16: CMBT summary of 28-day expansion results for virgin Sudbury aggregate

\subsubsection{CMBT Results for Sudbury RCA-M}

CMBT expansion results of Sudbury RCA-M containing GU Portland cement and varying levels of fly ash can be seen in Figure 4.1.17.

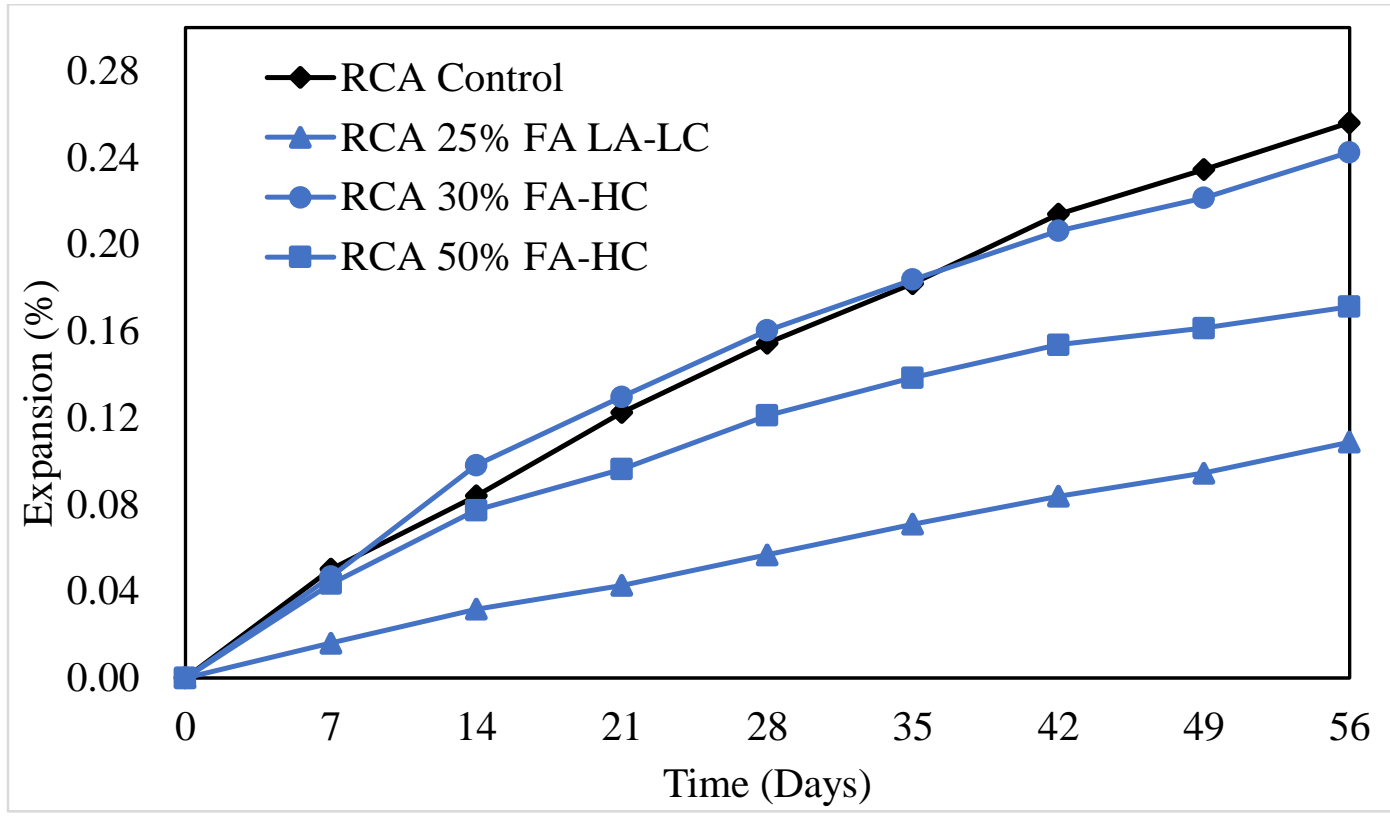

Figure 4.1.17: CMBT Expansion results of Sudbury RCA control and Sudbury RCA containing fly ash 
When looking at the data obtained for Sudbury RCA-M with GU Portland cement, as a control mix and with the use of fly ash, all 4 mixes produce samples that exceed the $0.04 \%$ expansion limit at 28 days. The closest mix is the mix containing $25 \%$ FA LA-LC, expanding $0.06 \%$ at day 28 and $0.11 \%$ at day 56 , followed by the mix containing $50 \%$ FA-HC $(0.12 \%$ at day 28 and $0.17 \%$ at day 56). Similar results were found in the other two mixed, with $30 \%$ FA-HC producing $0.16 \%$ expansion at day 28 and $0.24 \%$ expansion at day 56 and the control mix producing $0.15 \%$ expansion at day 28 and $0.26 \%$ expansion at day 56. The expansion in the mix containing $30 \%$ FA-HC has exceeded the expansion of the control mix again, which also occurred in the virgin Sudbury microbar samples. It seems, as it did with the virgin Sudbury mix that the control mix experienced less than expected expansion over the first 21 days, in which the expansion began to pick up to what would eventually lead it to expand more than the $20 \%$ FA-HC mix by the week 6 reading.

When introducing slag in Sudbury RCA-M microbars containing GU Portland cement, the microbars consisting of 50\% slag produced expansion results just below the $0.04 \%$ limit at 28 days and had an expansion of $0.08 \%$ at 56 days, shown in Figure 4.1.18. The mixes containing $40 \%$ slag and $30 \%$ slag followed, with the $40 \%$ slag expanding $0.06 \%$ at 28 days and $0.13 \%$ at 56 days and the $30 \%$ slag expanding $0.09 \%$ at 28 days and $0.18 \%$ at 56 days. 


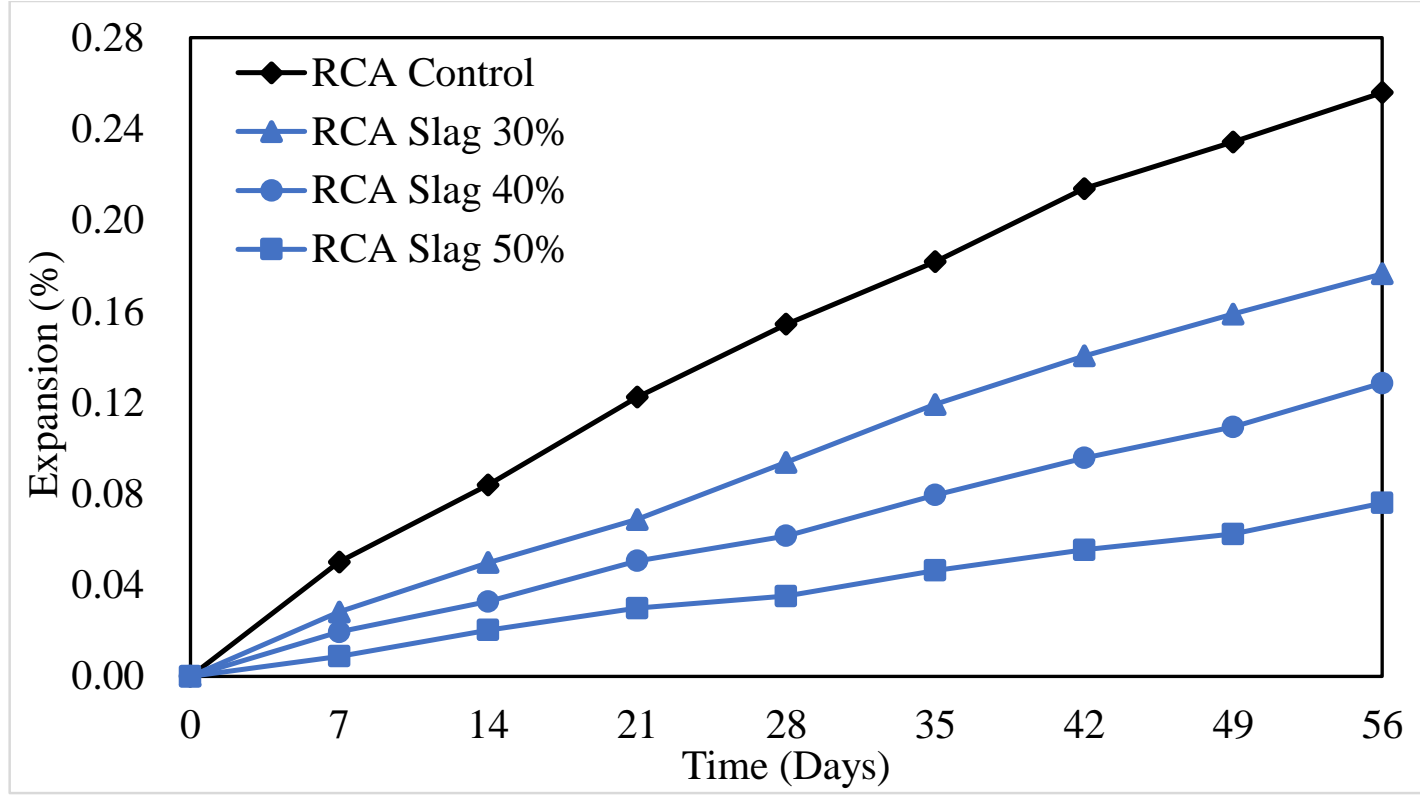

Figure 4.1.18: CMBT Expansion results of Sudbury RCA control and Sudbury RCA
containing slag

In the next set of results, the GU Portland cement was replaced with the HSF blended cement and again, the expansion results dropped significantly. In the mix containing HSF cement, the 28-day expansion of $0.10 \%$ and 56-day expansion of $0.20 \%$ are both $0.05 \%$ less than the 28 and 56-day expansion results of the control mix containing GU Portland cement. When fly ash and slag are incorporated into the mix along with the HSF blended cement, the expansion results continue to decrease, shown in Figure 4.1.19. The mixes containing HSF with 20\% FA-HC (0.07\% expansion at 28 days and $0.13 \%$ expansion at 56 days) and HSF with 30\% FA-HC (0.07\% expansion at 28 days and $0.12 \%$ expansion at 56 days) are the next two mixes of this set yielding the highest expansion, both of which do not satisfy the $0.04 \%$ expansion limit at 28 days. The other two mixes, containing HSF with 25\% FA LA-LC (expanding slightly below $0.04 \%$ at 28 days and $0.06 \%$ at 56 days) and HSF with $30 \%$ slag (expanding $0.03 \%$ at 28 days and $0.07 \%$ at 56 days) do satisfy the limit. 


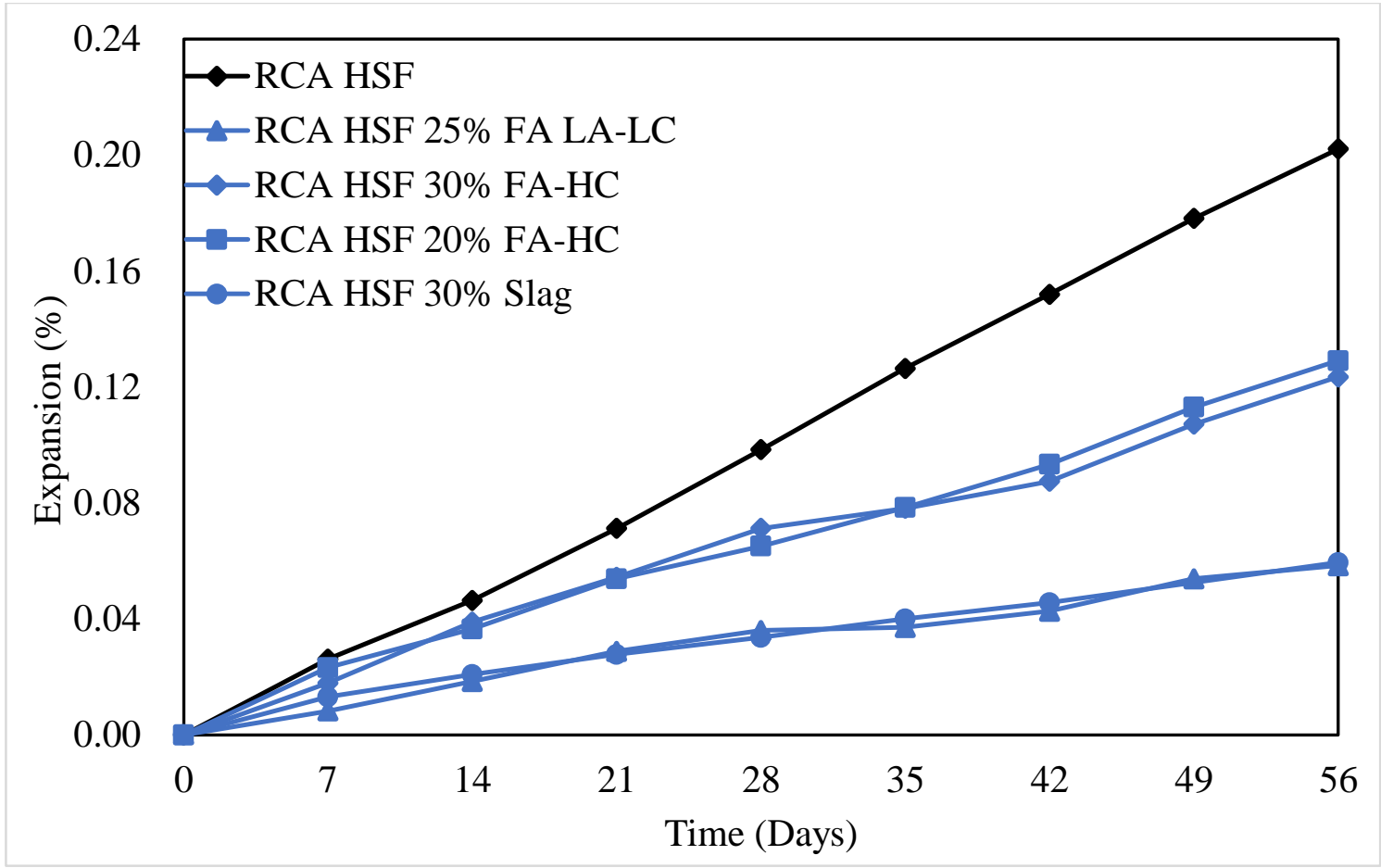

Figure 4.1.19: CMBT Expansion results of Sudbury RCA-M containing HSF and various SCM

Aside from the control mix, all mixes seem to follow a steady trend and contain no outliers, suggesting accurate readings were taken throughout. When comparing the 28-day expansion results of all the CMBT mixes containing Sudbury RCA-M, shown in Figure 4.1.20, it can be seen that there are three mixes (GU Portland cement with 50\% slag, HSF with 25\% FA-HC, and HSF with $30 \%$ slag) that satisfy the $0.04 \%$ expansion limit at 28 days for the CMBT, though all three of the mixes are very close to the limit. This suggests that they should be further tested through the CPT in order to verify the results obtained. The mixes containing $25 \%$ FA LA-LC, $40 \%$ slag, HSF with $30 \%$ FA-HC, and HSF with $20 \%$ FA-HC are slightly above the limit, thus further testing may prove them to be satisfactory. The rest of the mixes are far off the limit and can likely be assumed to be unsafe for use. 


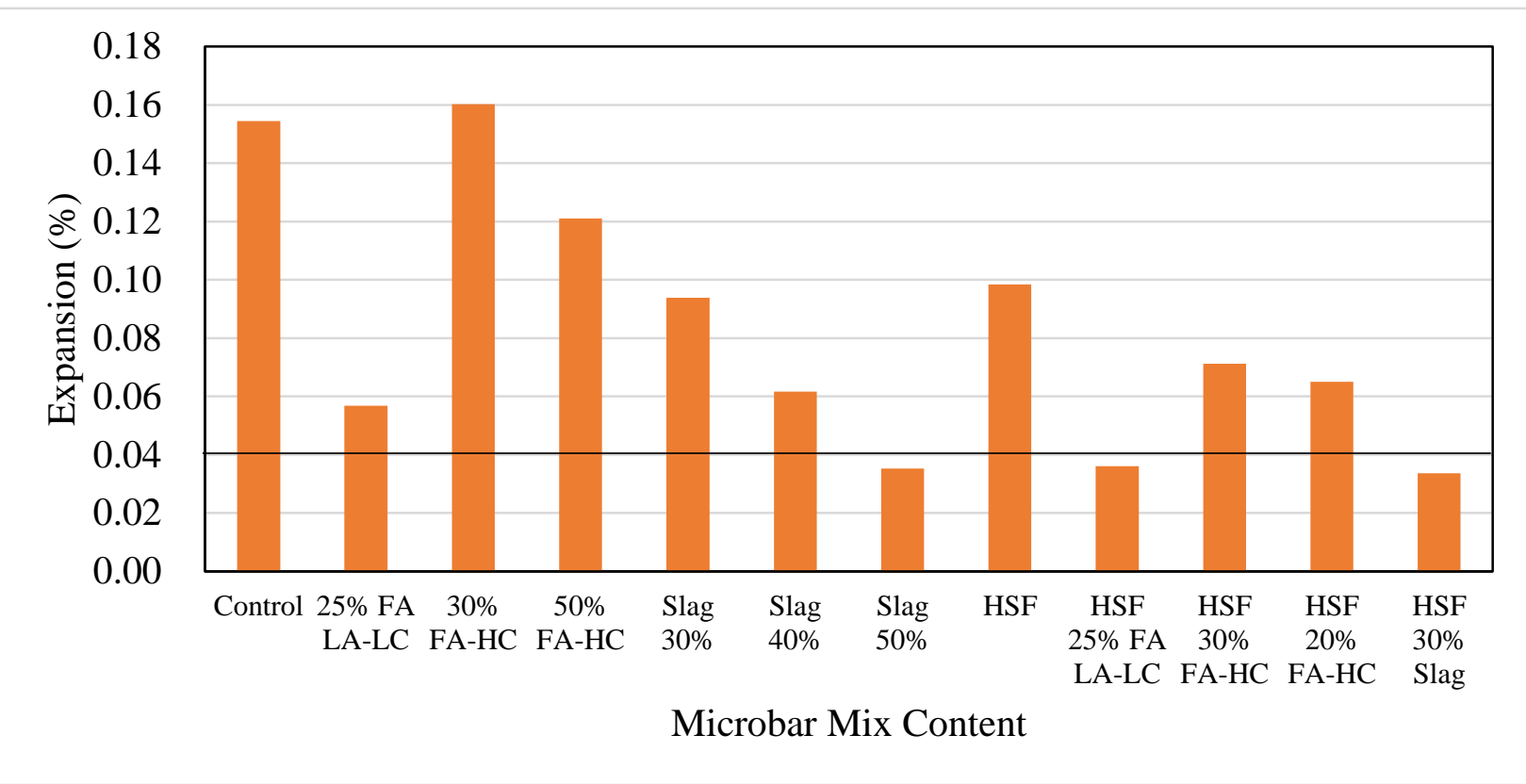

Figure 4.1.20: CMBT summary of 28-day expansion results for Sudbury RCA-M

\subsubsection{Comparing CMBT Results of Virgin Sudbury Aggregate and Sudbury RCA-M}

When looking at the summary of results at 28 days for the CMBT for both virgin Sudbury aggregate and Sudbury RCA-M, it is clear that the Sudbury RCA-M expands at a higher rate than that of the virgin aggregate, shown in Figure 4.1.21. There are 5 mixes that satisfy the $0.04 \%$ limit for RCA-M while there are 7 for the virgin aggregate. It is likely that expansion in the control mixes for both aggregates should be slightly higher, above the mixes containing 30\% FA-HC, as the expansion of those two sets of samples appeared slower over the first 28 days of measuring. 


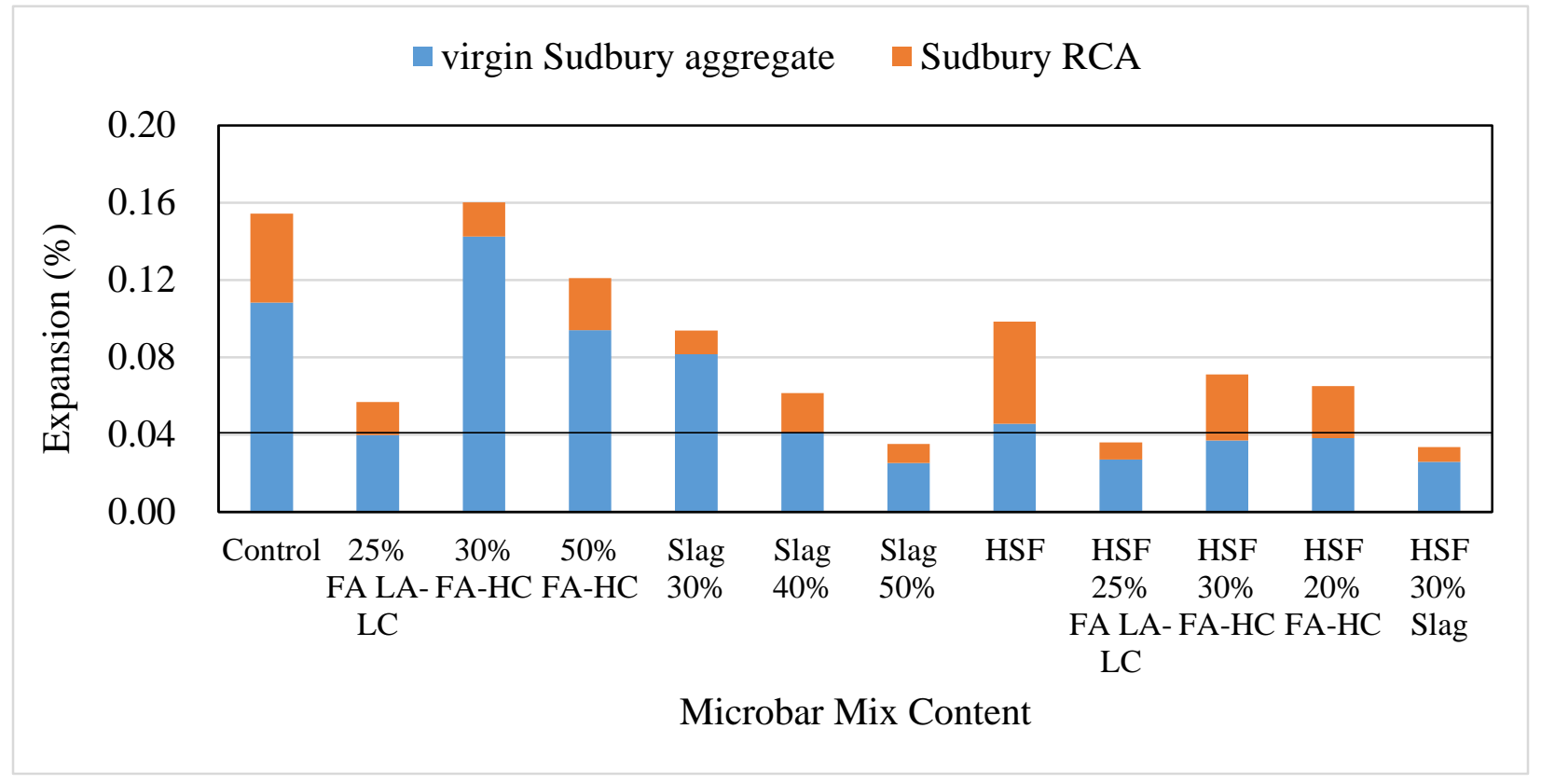

Figure 4.1.21: CMBT Summary of 28-day expansion results

When looking at the summary of results for 56-day expansion of the CMBT, shown in Figure 4.1.22, the same trends hold with all RCA-M mixes providing higher expansion results than that of the virgin aggregate.

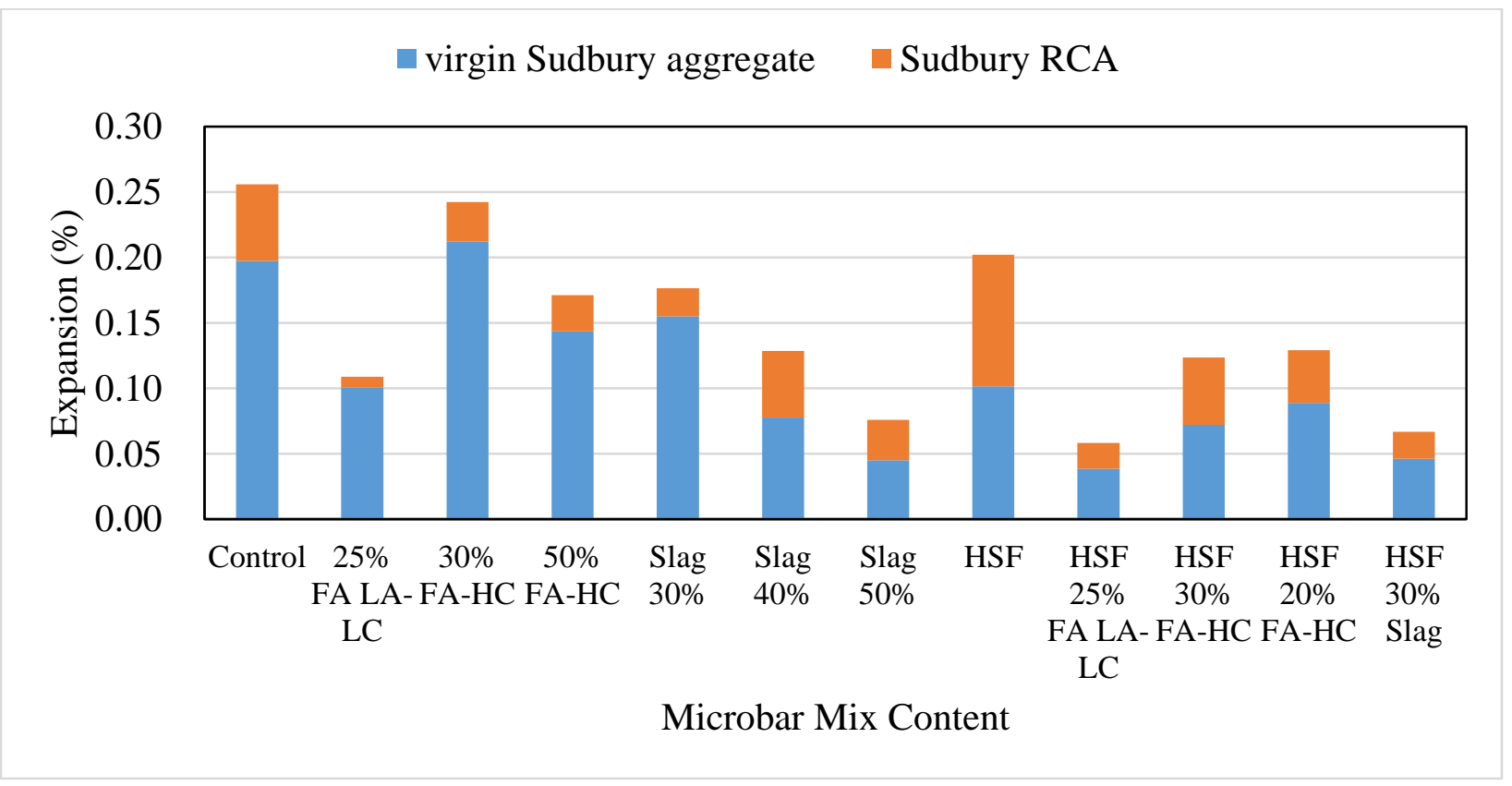

Figure 4.1.22: CMBT Summary of 56-day expansion results 
Figure 4.1.22 further verifies that the problem likely resides in the mix containing the virgin Sudbury aggregate. Thus, it can be concluded that the CMBT suggests that mixes containing Sudbury RCA expand at a higher rate than mixes containing virgin Sudbury aggregate. It should be noted that the effectiveness of each SCM continues to stay consistent between the AMBT and CMBT. Again, the most effective SCM in reducing expansion is the low-calcium fly ash, followed by the slag, leaving the high-calcium fly ash being the least effective. When used in combination with HSF, the effects are greater. Figure 4.1.2 shows a summary of expansion values for 28-day data of the CMBT.

Table 4.1.2: Summary of 28-day expansion data for CMBT

\begin{tabular}{|c|c|c|}
\hline \multirow{2}{*}{ Mix Type } & \multicolumn{2}{|c|}{ 28-day expansion value (\%) } \\
\cline { 2 - 3 } & $\begin{array}{c}\text { Virgin Sudbury } \\
\text { Aggregate }\end{array}$ & Sudbury RCA-M \\
\hline Control & 0.108 & 0.154 \\
\hline $25 \%$ FA LA-LC & 0.040 & 0.057 \\
\hline $30 \%$ FA-HC & 0.142 & 0.160 \\
\hline $50 \%$ FA-HC & 0.094 & 0.121 \\
\hline $30 \%$ Slag & 0.082 & 0.094 \\
\hline $40 \%$ Slag & 0.041 & 0.062 \\
\hline $50 \%$ Slag & 0.025 & 0.035 \\
\hline HSF (8\% Silica Fume) & 0.046 & 0.098 \\
\hline HSF w/ 25\% FA LA-LC & 0.027 & 0.036 \\
\hline HSF w/ 30\% FA-HC & 0.037 & 0.071 \\
\hline HSF w/ 20\% FA-HC & 0.038 & 0.065 \\
\hline HSF w/ 30\% Slag & 0.051 & 0.034 \\
\hline
\end{tabular}




\subsubsection{Concrete Prism Test}

The concrete prism test (CPT) was conducted for the same 12 mixes containing virgin Sudbury aggregate and Sudbury RCA-M as the AMBT and CMBT to verify the results. An aggregate is deemed non-reactive if the expansion of the samples is less than $0.04 \%$ after 1 year for mixes containing no SCM and $0.04 \%$ after 2 years for mixes containing SCM (CSA A23.2-28A, 2009). An aggregate is labelled moderately reactive with one year expansion between $0.04 \%-0.12 \%$, highly reactive with one year expansion greater than $0.12 \%$, and extremely reactive with one year expansion greater than $0.23 \%$. Currently, results have only been obtained up to 1 year and 3 months for mixes containing SCM, thus results are not complete.

\subsubsection{CPT Results for Virgin Sudbury Aggregate}

The first series of data for the CPT with virgin Sudbury aggregate consists of mixes containing GU Portland cement and different levels and portions of fly ash. The control mix, containing only GU Portland cement, expanded $0.168 \%$ after 1 year, which classifies Sudbury aggregate as highly reactive $(>0.12 \%)$. When introducing fly ash into the mix, the expansion reduces significantly, shown in Figure 4.1.23. At week 65 (1 year and 3 months), all three mixes contain expansion values below the two year limit, led by the mix containing 50\% FA-HC with an expansion of $0.01 \%$. The mixes containing $25 \%$ FA HA-LC and $30 \%$ FA-HC trail, with an expansion of $0.03 \%$. It is important to note that the low-calcium fly ash used in the CPT mixes, which contains 3.68\% $\mathrm{Na}_{2} \mathrm{O}_{\mathrm{e}}$, is different from than the one used in the AMBT and CMBT, thus the CPT shall be redone using FA LA-LC. It seems that the control mix has 2 slight outliers at weeks 13 and 18, however they seem to be measuring errors as the curve appears to follow the same trend set out in the first 8 weeks of measurements. 


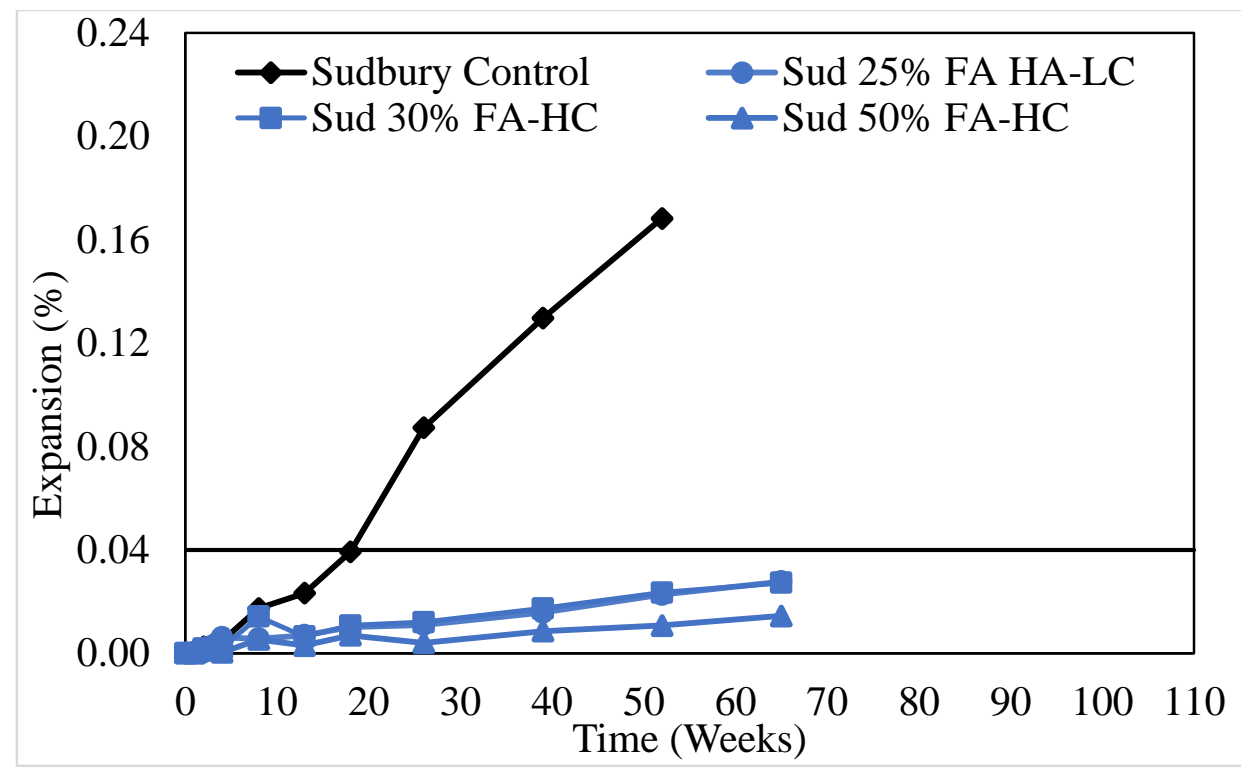

Figure 4.1.23: CPT Expansion results of Sudbury control and Sudbury containing fly ash

With the introduction of varying levels of slag, similar results prevail. The expansion in the mixes containing $30 \%, 40 \%$, and $50 \%$ slag are drastically less than that of the control mix, shown in Figure 4.1.24. The 65 week expansion results indicate an expansion of $0.02 \%$ for the mixes containing 30\% slag and $40 \%$ slag and $0.01 \%$ expansion for the mix containing 50\% slag, all of which are currently below the 2-year limit of $0.04 \%$.

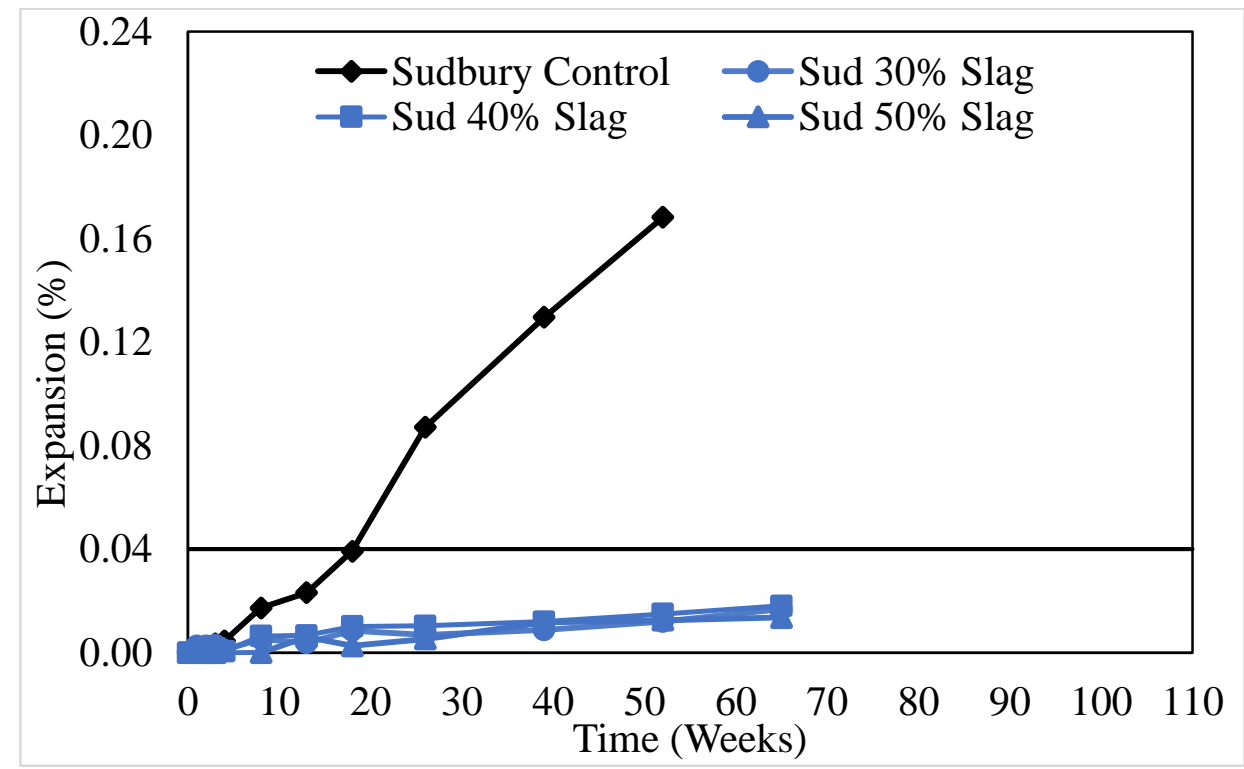

Figure 4.1.24: CPT Expansion results of Sudbury control and Sudbury containing slag 
When replacing the HSF blended cement with the GU Portland cement, the expansion reduces significantly $(0.02 \%$ at week 65$)$. When the addition of fly ash and slag are introduced to the mixes with HSF, the expansion continues to reduce, shown in Figure 4.1.25. The HSF mix experiences a slight jump in expansion at week 13 , however comes back down to follow the normal trend the rest of the way, suggesting a poor reading was taken. The mix containing HSF with $30 \%$ slag trails the HSF mix slightly, with an expansion just below $0.02 \%$ at week 65 , followed by the mixes containing HSF with 25\% FA HA-LC and HSF with $20 \%$ FA-HC, with an expansion of $0.08 \%$ at week 65. The mix containing HSF with 30\% FA-HC has shown almost no expansion through 65 weeks, expanding $0.0003 \%$. The 65 week expansion of all mixes containing HSF blended cement rather than GU Portland cement are well below the 2-year limit of $0.04 \%$. The only mix that may be in danger of exceeding the limit at year 2 is the mix containing only HSF.

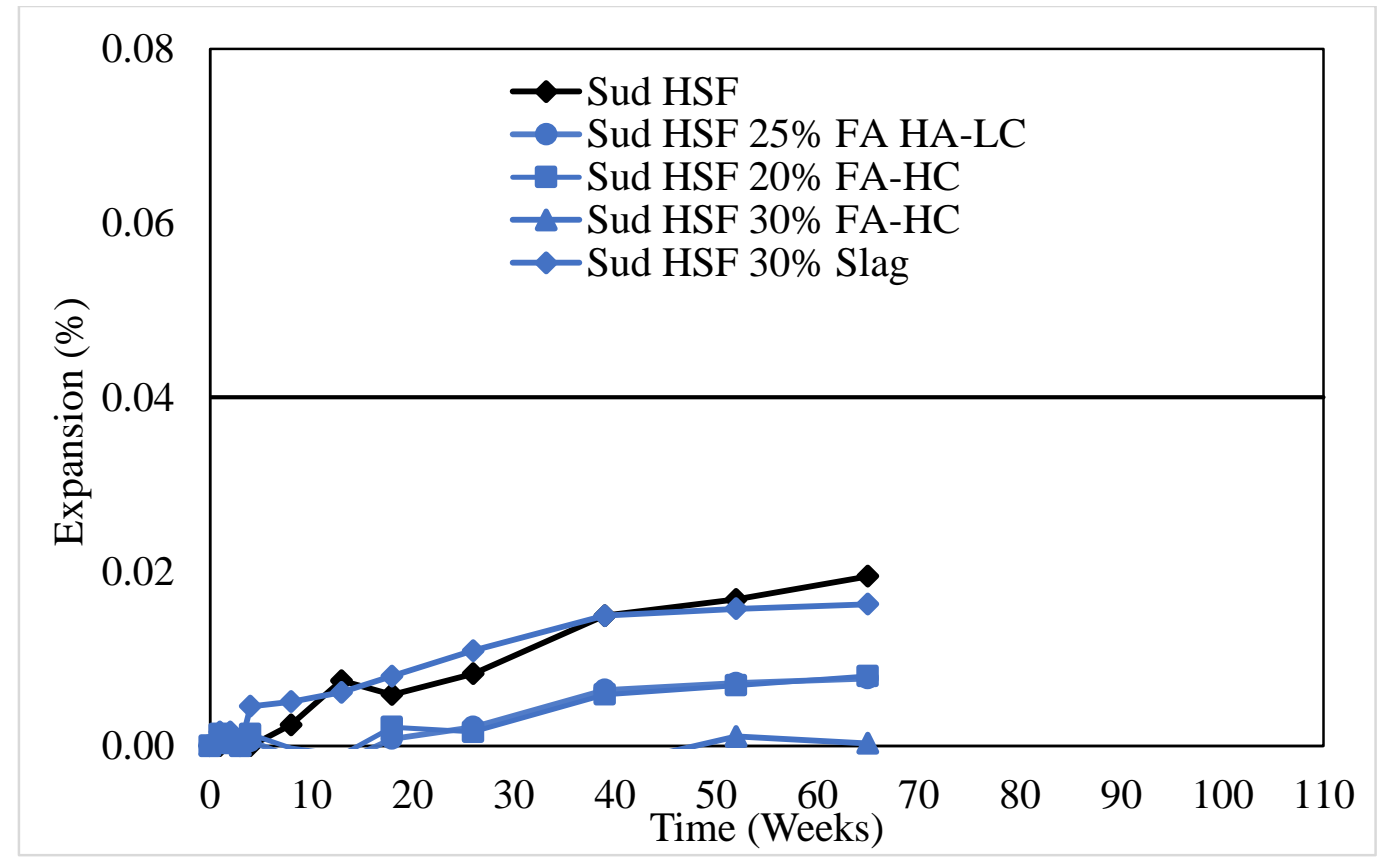

Figure 4.1.25: CPT Expansion results of Sudbury containing HSF and various SCM

When comparing the results at 1 year and 3 months (1 year for Sudbury control mix) of all mixes completed for the CPT containing virgin Sudbury aggregate, shown in Figure 4.1.26, it is clear 
that the use of SCM has an incredibly high impact. After 65 weeks, none of the mixes containing any form of SCM has surpassed the $0.04 \%$ limit for 2 years. Although it is clear that some mixes will surpass the limit, such as the mix containing 25\% FA HA-LC and 30\% FA-HC, it is also apparent that many will stay below the limit. The data will be further monitored until 2-year data is obtained before recommendations are given on the level and type of SCM required to sufficiently mitigate ASR in virgin Sudbury aggregate.

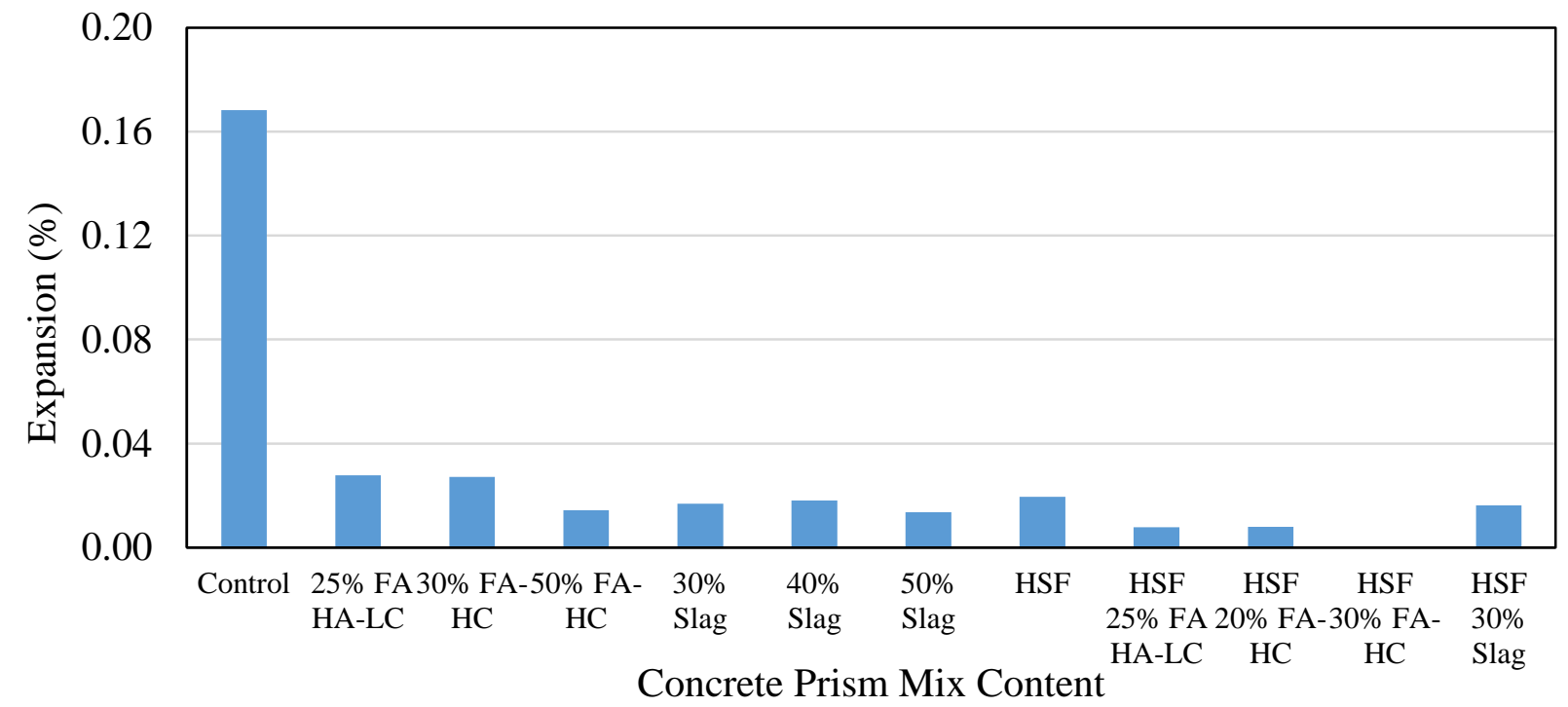

Figure 4.1.26: CPT summary of 65-week (1 year for Control) expansion results for virgin Sudbury aggregate

\subsubsection{CPT Results for Sudbury RCA-M}

When the CPT test was used with Sudbury RCA-M, the first set of samples consisted of GU Portland cement and fly ash. The control mix provided a 1-year expansion value of $0.21 \%$, classifying the aggregate as a highly reactive aggregate. When fly ash was introduced, the expansion reduced immensely, much like that of the virgin Sudbury aggregate. The 25\% FA HCLC mix provided an expansion of $0.06 \%$ at week 65 , followed by $0.05 \%$ expansion for the $30 \%$ FA-HC mix and $0.03 \%$ expansion for the $50 \%$ FA-HC mix. Of the three mixes, the only mix still 
below the $0.04 \%$ limit at 2 years is the mix containing 50\% FA-HC, although it is likely to surpass the limit before 2 years.

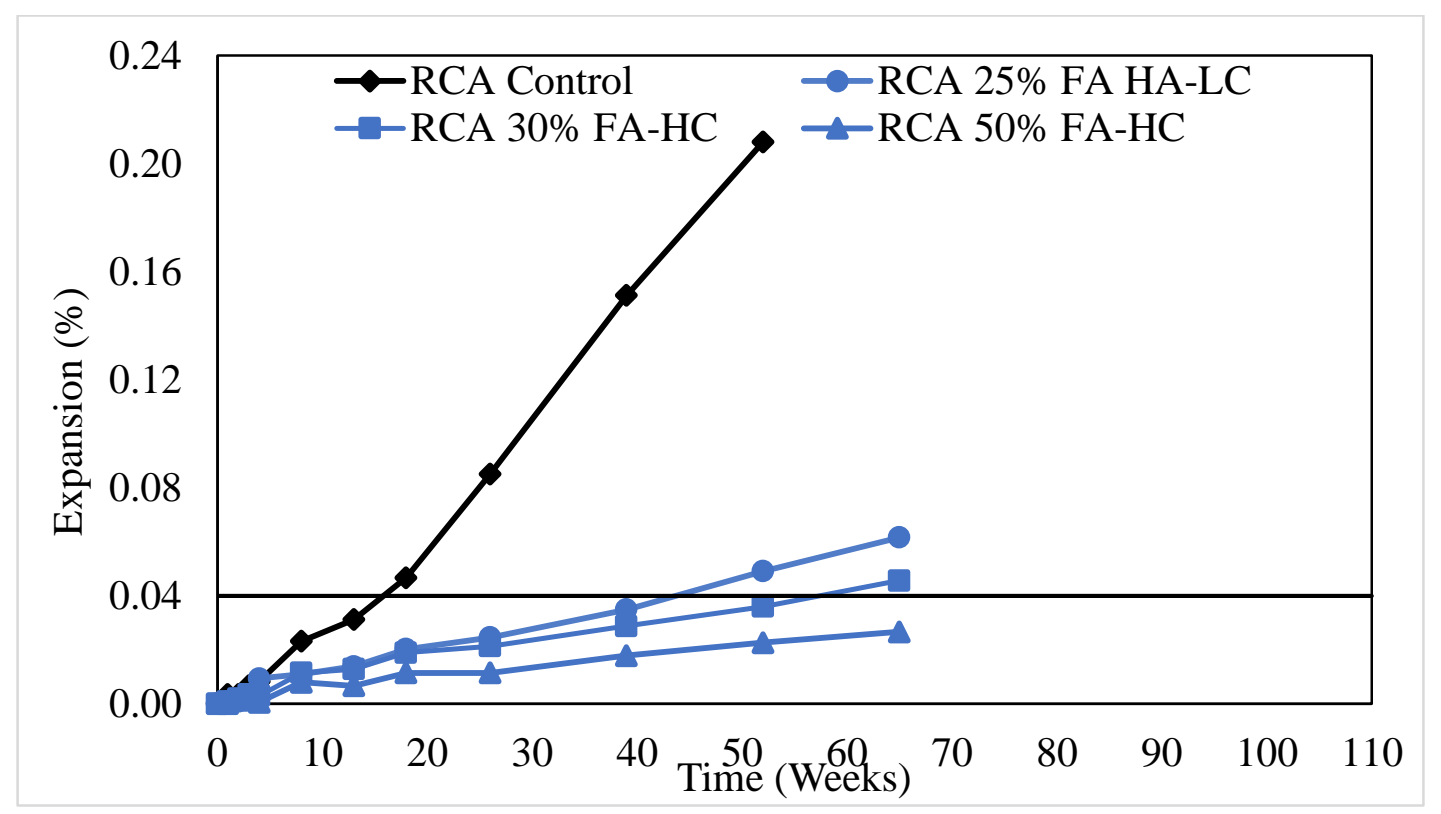

Figure 4.1.27: CPT Expansion results of Sudbury RCA control and Sudbury RCA containing fly ash

With the introduction of slag, the same trend occurred in that the use of SCM greatly reduces the expansion in comparison to the control mix. Figure 4.1 .28 shows that with $30 \%, 40 \%$, ad $50 \%$ slag introduced as a replacement to GU Portland cement, the expansion at 65 weeks is currently below the 2 year limit of $0.04 \%$. That being said, the mixes containing $30 \%$ and $40 \%$ slag are all likely to exceed the limit, as they have expansion values of $0.03 \%$ at week 65 and the mix containing $50 \%$ slag will be very close to the limit, as its 65 week expansion is $0.02 \%$. 


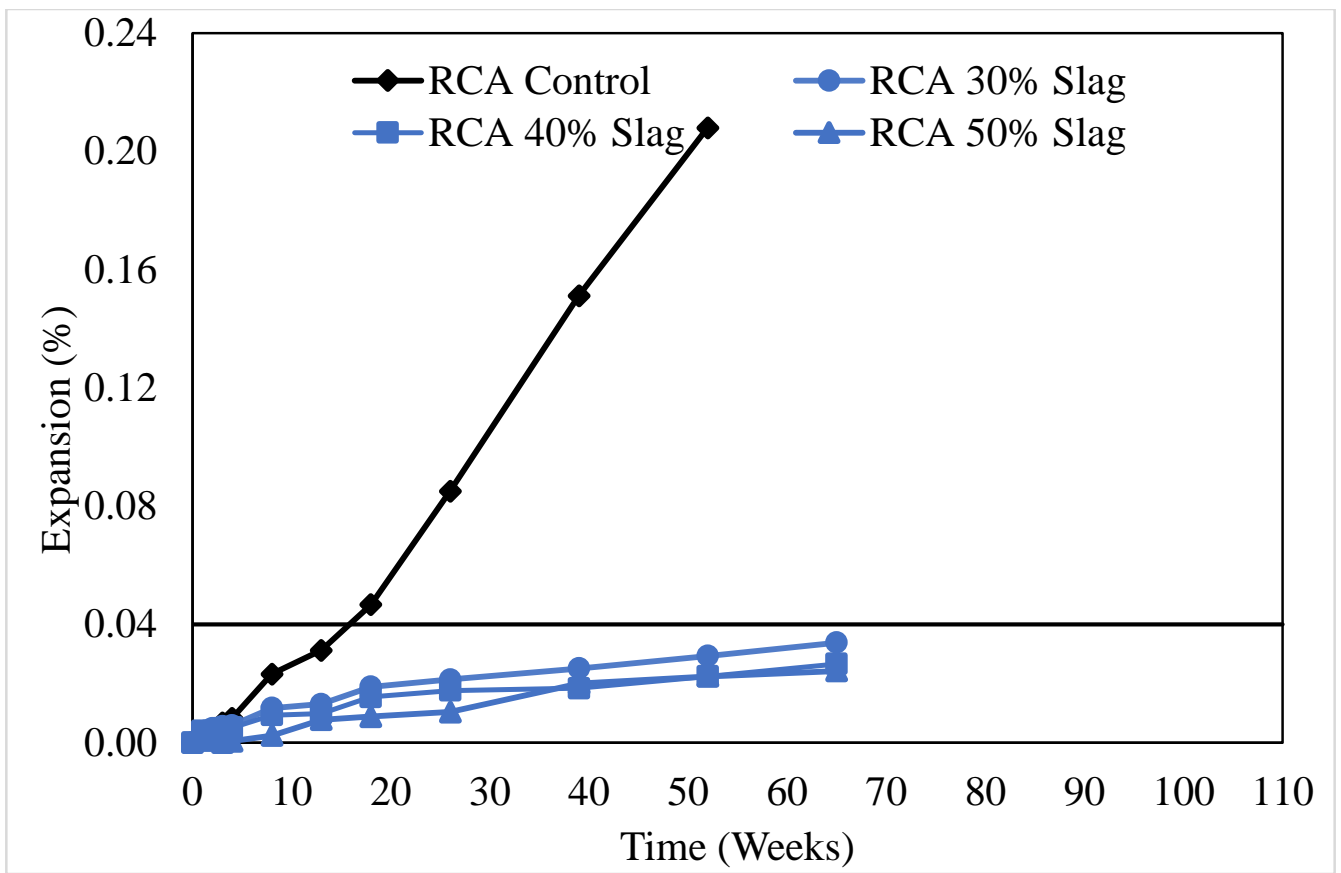

Figure 4.1.28: CPT Expansion results of Sudbury RCA control and Sudbury RCA containing slag

When replacing GU Portland cement with HSF blended cement, the HSF mix drops to 0.03\% expansion at 65 weeks (compared to the GU Portland cement control mix of $0.21 \%$ expansion at 52 weeks). With the introduction of SCM in addition to the HSF blended cement, the expansion continues to decrease, shown in Figure 4.1.29. As it did in the mix containing virgin Sudbury aggregate, the mix containing HSF experiences an odd jump at week 13, further suggesting a poor reading was taken as they were measured together at the same time. The mix containing HSF with $30 \%$ slag has a similar $0.03 \%$ week- 65 expansion, though seems to be slowing down in comparison to the HSF mix. The other three mixes are all just below $0.02 \%$ expansion at 65 weeks, with the mix containing HSF and 25\% FA HA-LC showing the least expansion. It appears that the HSF mixes containing 20\% FA-HC, 30\% FA-HC, and 25\% FA HA-LC will all remain below the $0.04 \%$ limit at year 2, while the mix containing only HSF and HSF with $30 \%$ slag mixes will be very close to the limit. 


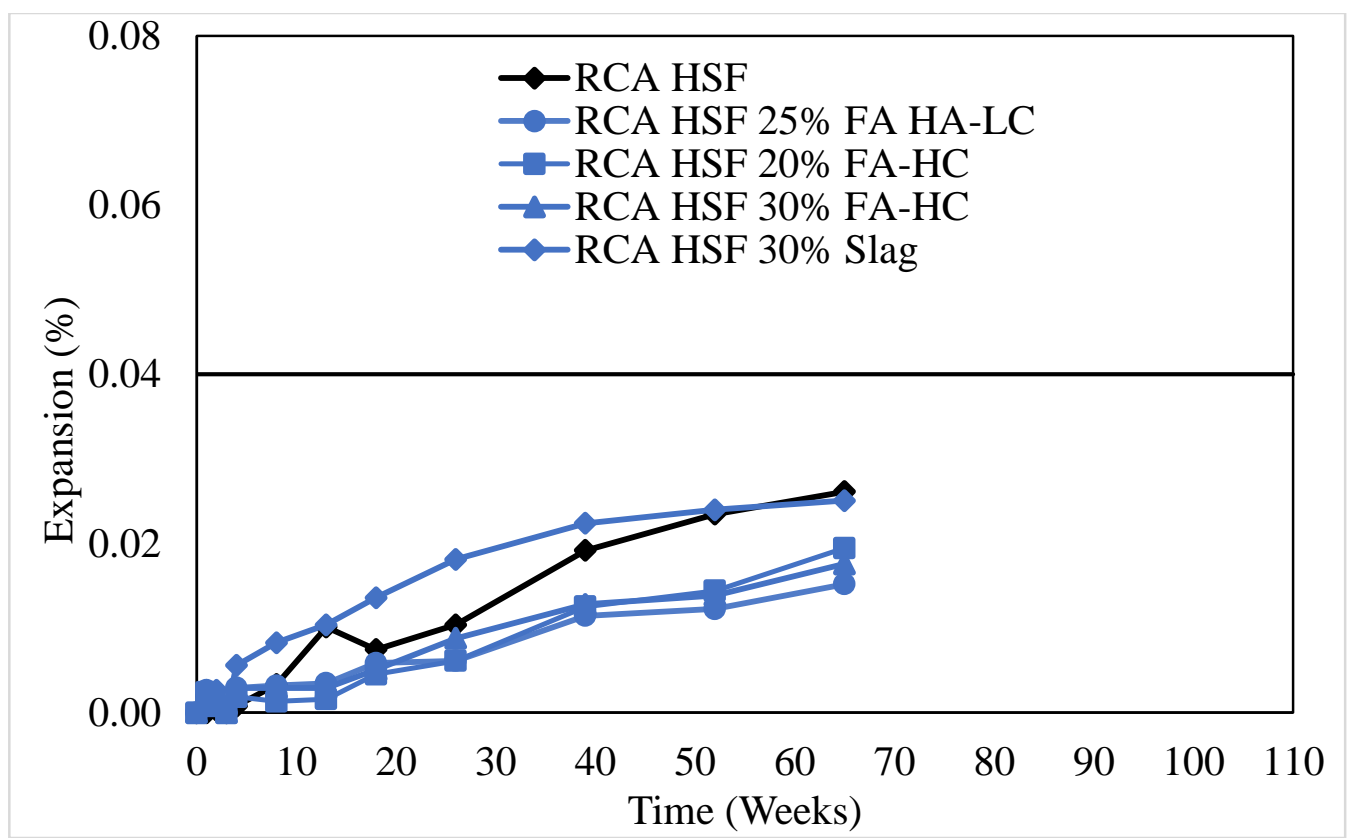

Figure 4.1.29: CPT Expansion results of Sudbury RCA-M containing HSF and various SCM

When looking at the 1 year and 3 month ( 1 year for the control mix) data for the mixes containing Sudbury RCA-M, it is clear that the same trend prevails as that of the virgin Sudbury aggregate. That is, that the use of any SCM significantly reduces the expansion of the CPT, shown in Figure 4.1.30. It can be seen that 3 mixes (control, 25\% FA HA-LC, and 30\% FA-HC) have already surpassed the $0.04 \%$ limit, and that it is likely only 3 or 4 mixes remain below the limit by the time the 2 year reading is taken. 


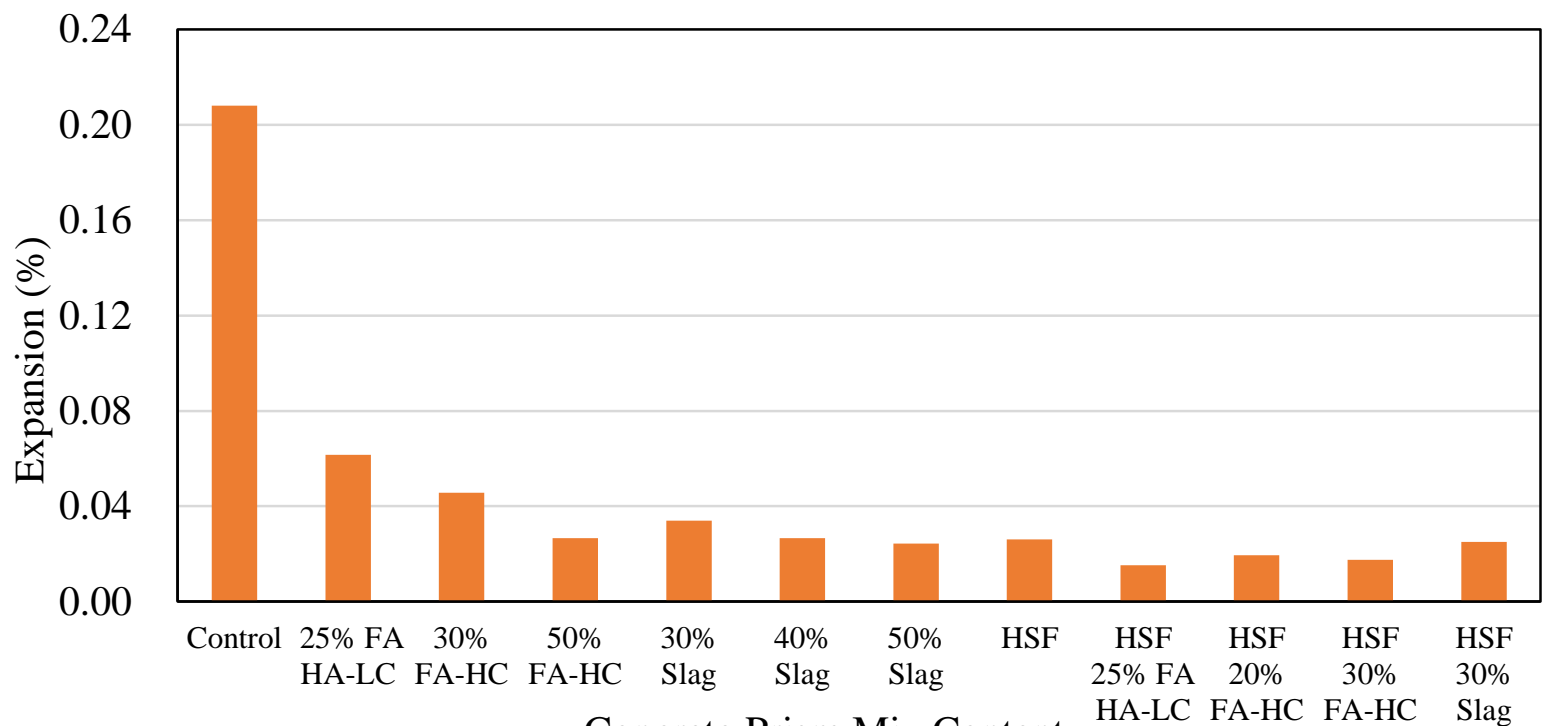

Concrete Prism Mix Content

Figure 4.1.30: CPT summary of 65-week (1 year for Control) expansion results for Sudbury RCA-M

\subsubsection{Comparing CPT Results of Virgin Sudbury Aggregate and Sudbury RCA-M}

When looking at the summary of results at 65 weeks ( 1 year for control mixes) for the CPT for both virgin Sudbury aggregate and Sudbury RCA-M, it is clear that the Sudbury RCA-M expands at a higher rate than that of the virgin aggregate, shown in Figure 4.1.31. This case holds true for all mixes, which was the same trend that had occurred in the CMBT. Thus, it can be concluded that the Sudbury RCA-M is more reactive than that of the virgin Sudbury aggregate. It is important to note that the most effective SCM used in the CPT was the slag, followed by FA HA-LC and FA-HC. The SCM were even more effective when used in combination with HSF blended cement. This differs from the findings in the CMBT and AMBT, as the FA LA-LC was found to be the most effective. However the low-calcium fly ash used in those mixes contained a lower alkali fly ash, thus it is likely the same trend would have occurred given the use of FA LA-LC instead of FA HA-LC in the CPT. 


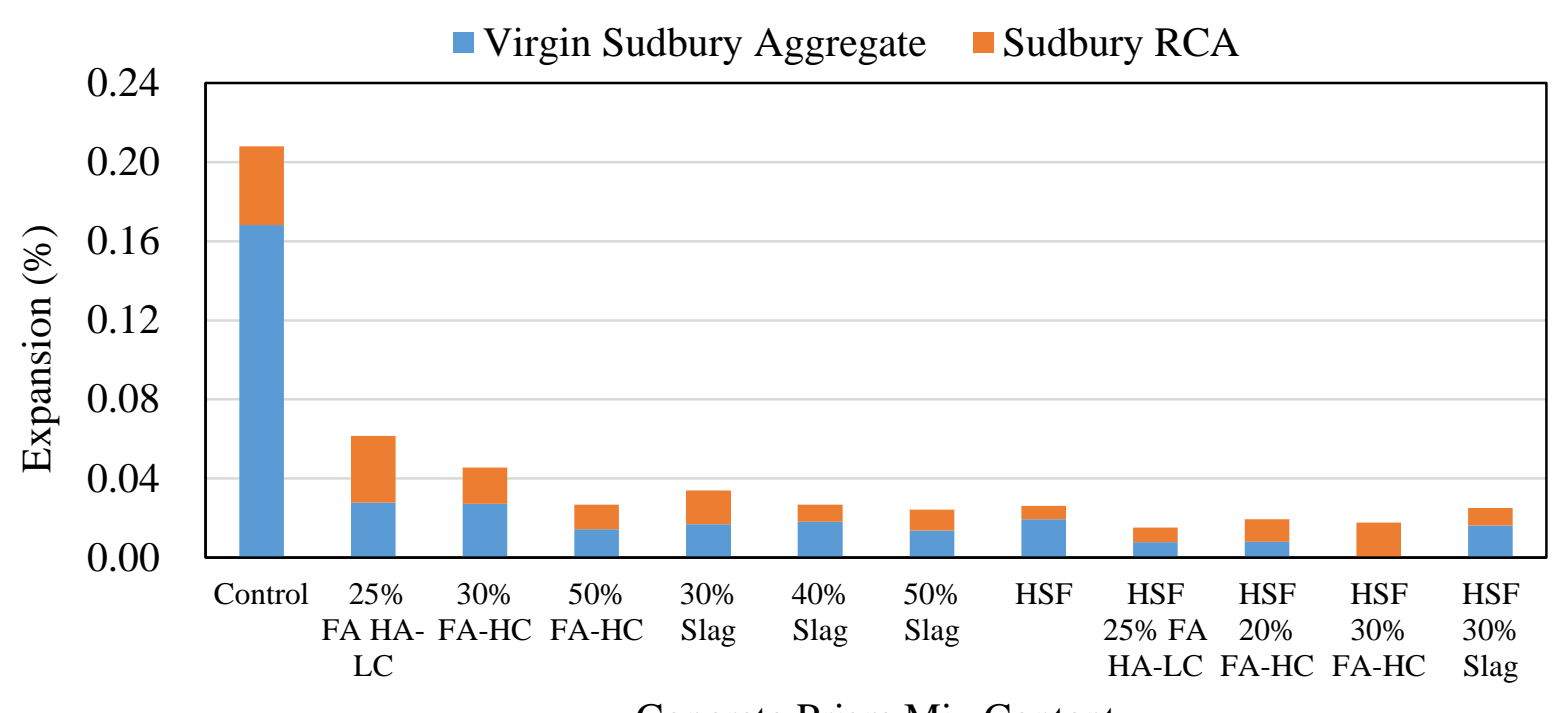

Concrete Prism Mix Content

Figure 4.1.31: CPT Summary of 65 week (1 year for control mixes) expansion results

Using linear extrapolation of the last 2 data points collected (week 52 and 65) the expansion data for all samples containing SCM have been interpreted for 2-year data. This was done by simply taking the slope over the last two readings and applying it to the end of the data to estimate the expansion at 2 years. It is important to keep in mind that the 2 -year data is extrapolated, thus it is what the expected expansion values are, not the actual expansion values. Figure 4.1.32 shows the 1-year expansion results of the control mixes and the expected 2-year expansion results of the mixes containing SCM for both virgin Sudbury aggregate and RCA-M. 


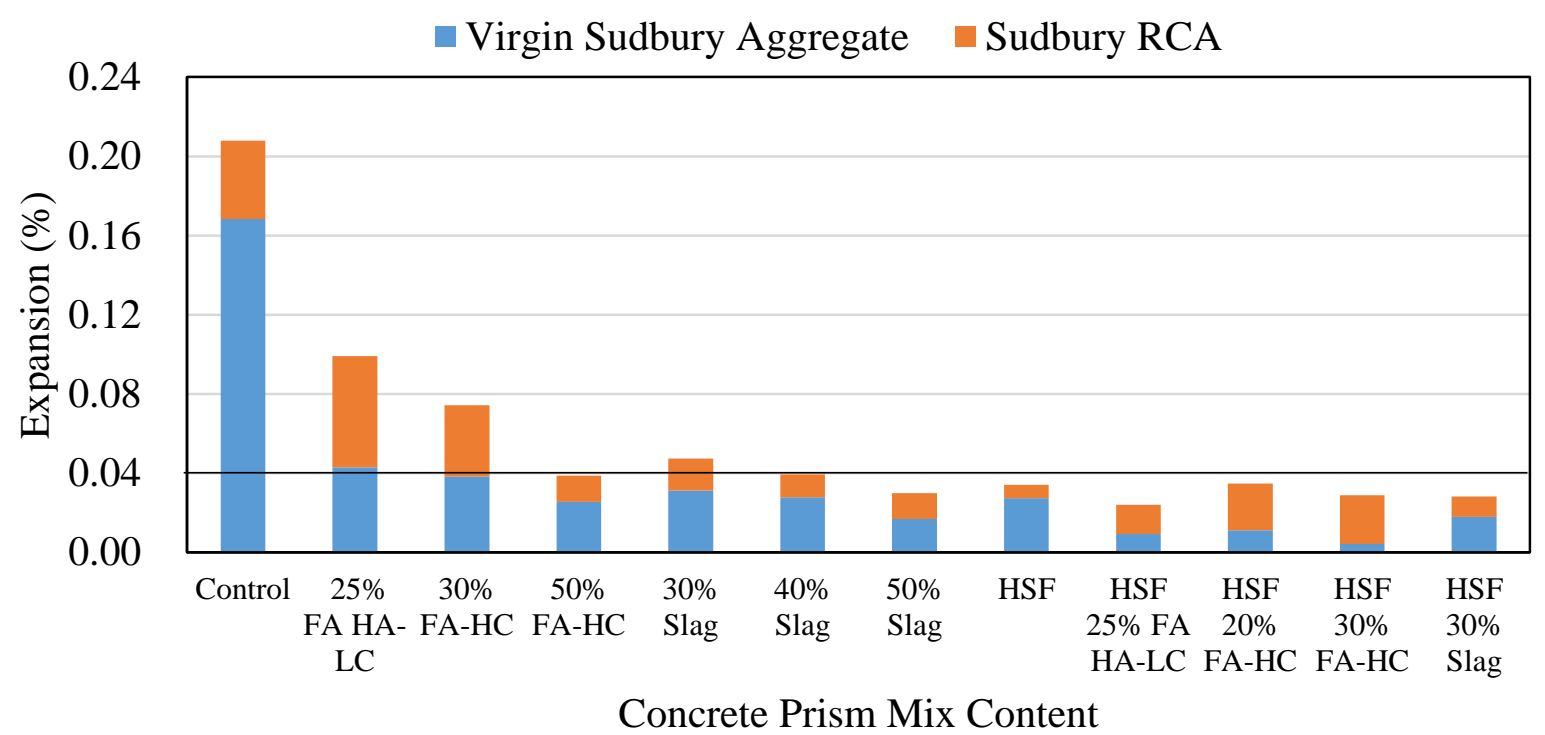

Figure 4.1.32: 1-year expansion results for control mixes containing GU Portland cement and expected 2-year expansion results for mixes containing SCM

With this extrapolation shown in Figure 4.1.32, it is expected that 9 of the mixes containing virgin Sudbury aggregate will remain below $0.04 \%$ expansion after 2 years while only 6 of the mixes containing Sudbury RCA-M will remain below the limit. A summary of the 1-year and expected 2-year expansion results are shown in Table 4.1.3.

Table 4.1.3: Summary of 1-year and expected 2-year expansion data for CPT

\begin{tabular}{|c|c|c|}
\hline \multirow{2}{*}{ Mix Type } & \multicolumn{2}{|c|}{$\begin{array}{c}\text { 1-year and expected 2-year* expansion } \\
\text { value (\%) }\end{array}$} \\
\cline { 2 - 3 } & $\begin{array}{c}\text { Virgin Sudbury } \\
\text { Aggregate }\end{array}$ & Sudbury RCA-M \\
\hline Control & 0.168 & 0.208 \\
\hline $25 \%$ FA HA-LC & $0.045^{*}$ & $0.099^{*}$ \\
\hline $30 \%$ FA-HC & $0.042^{*}$ & $0.070^{*}$ \\
\hline $50 \%$ FA-HC & $0.025^{*}$ & $0.042^{*}$ \\
\hline $30 \%$ Slag & $0.027^{*}$ & $0.046^{*}$ \\
\hline $40 \%$ Slag & $0.026^{*}$ & $0.036^{*}$ \\
\hline $50 \%$ Slag & $0.022^{*}$ & $0.038^{*}$ \\
\hline
\end{tabular}




\begin{tabular}{|c|l|l|}
\hline HSF (8\% Silica Fume) & $0.031^{*}$ & $0.042^{*}$ \\
\hline HSF w/ 25\% FA HA-LC & $0.013^{*}$ & $0.024^{*}$ \\
\hline HSF w/ 30\% FA-HC & $0.014^{*}$ & $0.033^{*}$ \\
\hline HSF w/ 20\% FA-HC & $0.005^{*}$ & $0.026^{*}$ \\
\hline HSF w/ 30\% Slag & $0.022^{*}$ & $0.032^{*}$ \\
\hline
\end{tabular}

*Denotes 2-year expansion value is expected through linear extrapolation of last 2 data points

\subsubsection{Comparing the AMBT and CPT Results}

After compiling the data from the AMBT and CPT, the graph shown in Figure 4.1.33 can be analyzed to compare the 14-day AMBT and expected 2-year CPT data for virgin Sudbury aggregate. The graph shows that 7 of the samples passed both the $0.15 \%$ limit at 14 -days of the AMBT and the $0.04 \%$ limit at 2-years for the CPT. This is the case for all 5 mixes containing HSF as well as the mix containing $50 \%$ FA-HC, $40 \%$ slag, and $50 \%$ slag. The mixes containing the different types of low-calcium fly ash are not shown in these series of graphs because their varying alkali contents cause different levels of expansion.

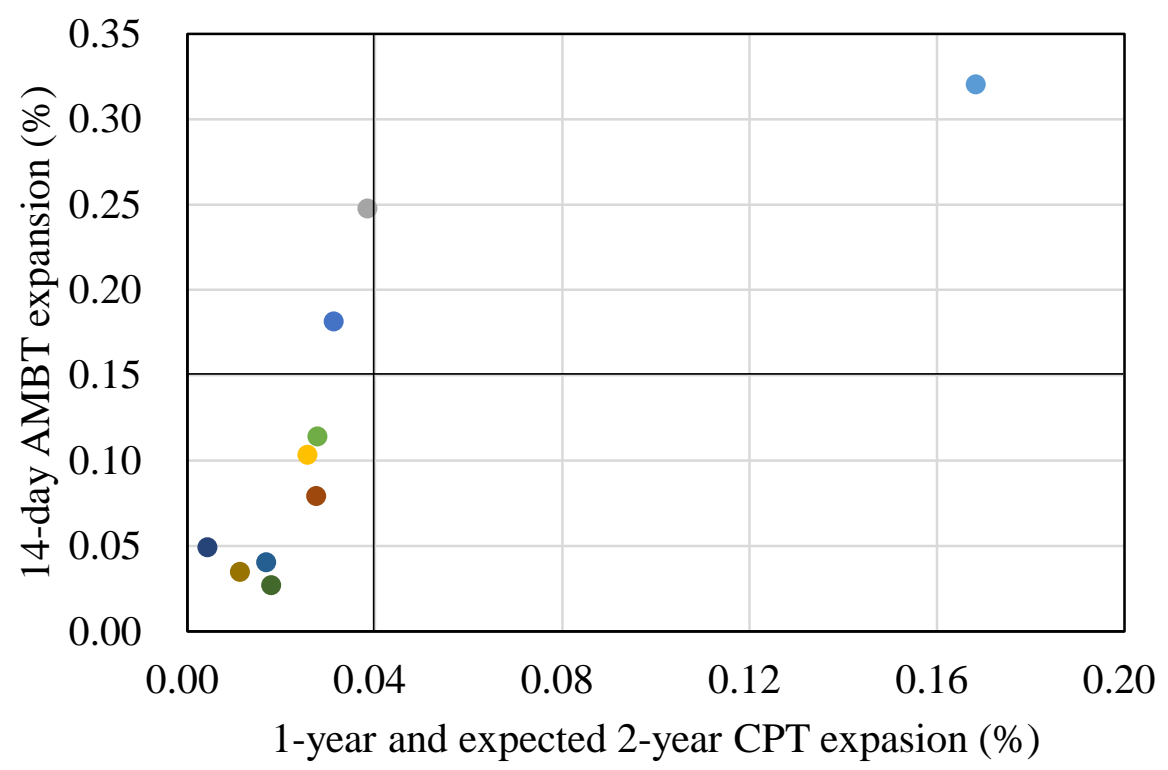

- Sudbury Control

- Sud $30 \%$ FA-HC

Sud $50 \%$ FA-HC

- Sud $30 \%$ Slag

- Sud $40 \%$ Slag

- Sud 50\% Slag

- Sud HSF

- Sud HSF $20 \%$ FA-HC

- Sud HSF $30 \%$ FA-HC

- Sud HSF 30\% Slag

Figure 4.1.33: 14-day AMBT vs. 1-year and expected 2-year CPT expansion for virgin Sudbury aggregate 
The data shown in Figure 4.1.34 compares the Sudbury RCA-M results of the AMBT and CPT. This graph shows that again, 7 samples pass both tests, including all samples containing HSF along with the mixes containing 50\% FA-HC, 40\% slag, and 50\% slag.

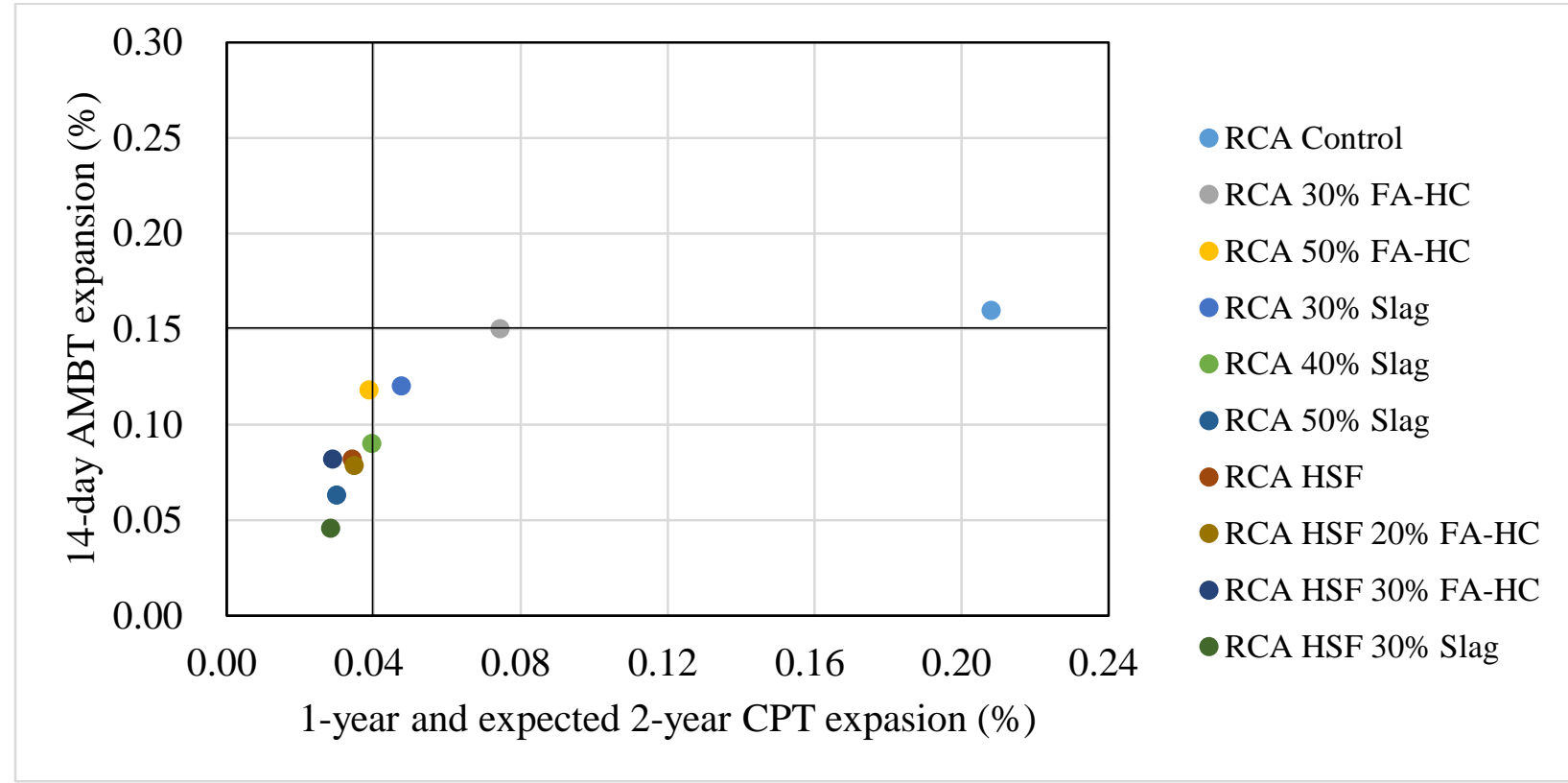

Figure 4.1.34: 14-day AMBT vs. 1-year and expected 2-year CPT expansion for Sudbury
RCA-M

\subsubsection{Comparing the CMBT and CPT Results}

The data comparing the 28-day expansion of the CMBT and the 2-year expected expansion of the CPT for virgin Sudbury aggregate can be seen in Figure 4.1.35. The graph shows that 4 of the samples passed both the $0.04 \%$ limit at 28 -days of the CMBT and the $0.04 \%$ limit at 2-years for the CPT. This is the case for the mixes containing HSF with 30\% FA-HC, HSF with 20\% FA-HC, HSF with $30 \%$ slag, as well as the mix containing 50\% slag. The mixes containing $40 \%$ slag and HSF expand slightly beyond the CMBT limit. Judging by the trend of the data points in Figure 4.1.35, it seems as though an expansion limit $0.10 \%$ is better suited for the CMBT for virgin 
Sudbury aggregate. With an expansion limit of $0.10 \%, 8$ of the 10 samples would pass both tests, with only 1 mix failing the CMBT while passing the CPT and 1 mix failing both. However, this observation needs to be verified when the two year-results of the concrete prisms are available. Alternatively, one can argue that the $0.04 \%$ expansion limit at 28 days provides a conservative expectation of the expansion in the concrete prism test.

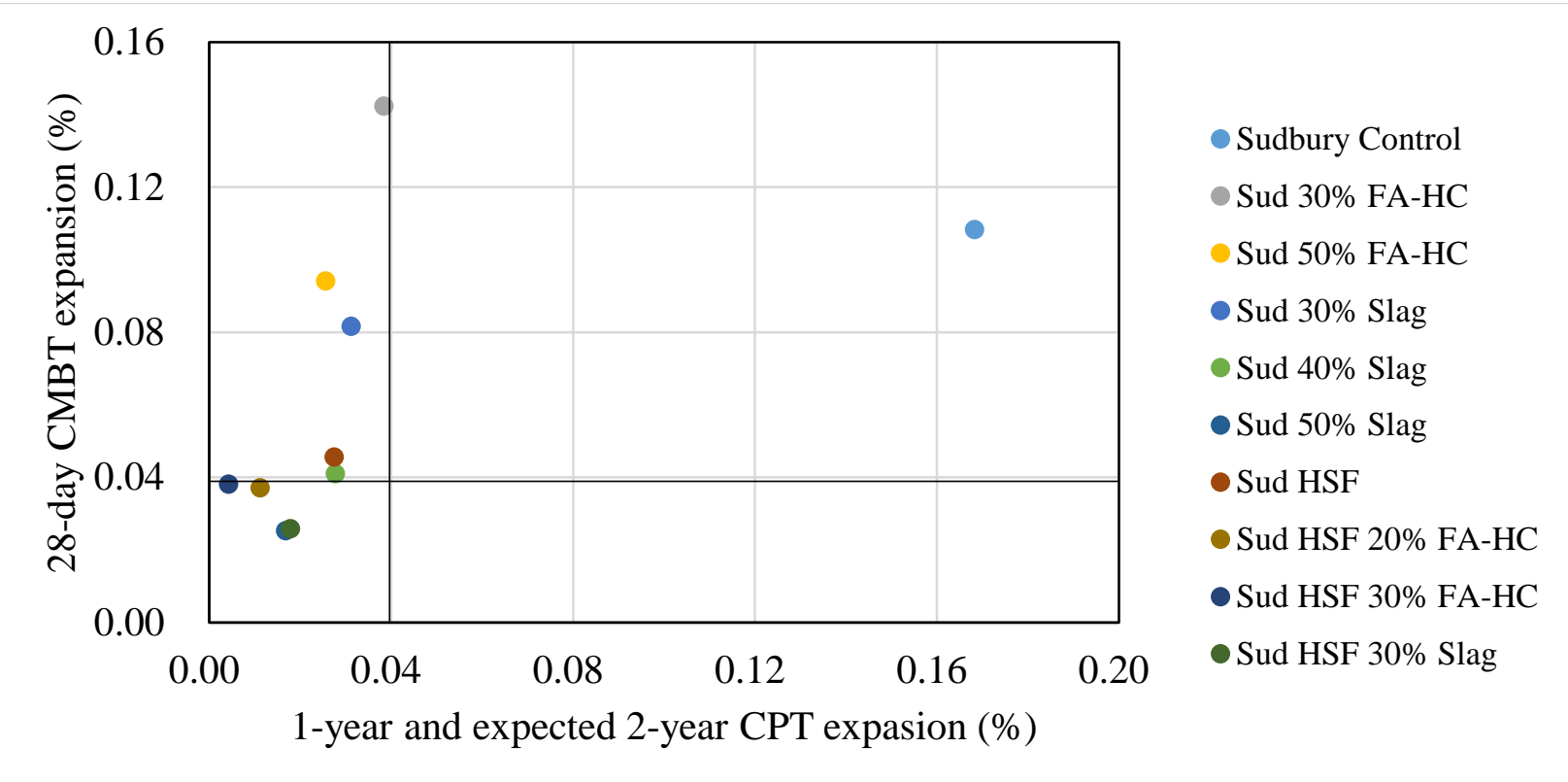

\section{Figure 4.1.35: 28-day CMBT vs. 1-year and expected 2-year CPT expansion for virgin Sudbury aggregate}

The data shown in Figure 4.1.36 compares the Sudbury RCA-M results of the CMBT and CPT at 28-days. This graph shows that only 2 samples pass both tests, including the mixes containing $50 \%$ slag and HSF with 30\% slag. All other mixes are significantly above the CMBT expansion limit. Judging by the trend of the data points in Figure 4.1.36, the same trend prevails as it seems as though an expansion limit of $0.10 \%$ is better suited for the CMBT for Sudbury RCA. With an expansion limit set to $0.10 \%$ at 28 days, 6 of the 10 samples would pass both tests, with only 1 
mix failing the CMBT while passing the CPT, 1 mix failing the CPT while passing the CMBT, and 2 mixes failing both. However, the same argument stated in Figure 4.1.35 above applies.

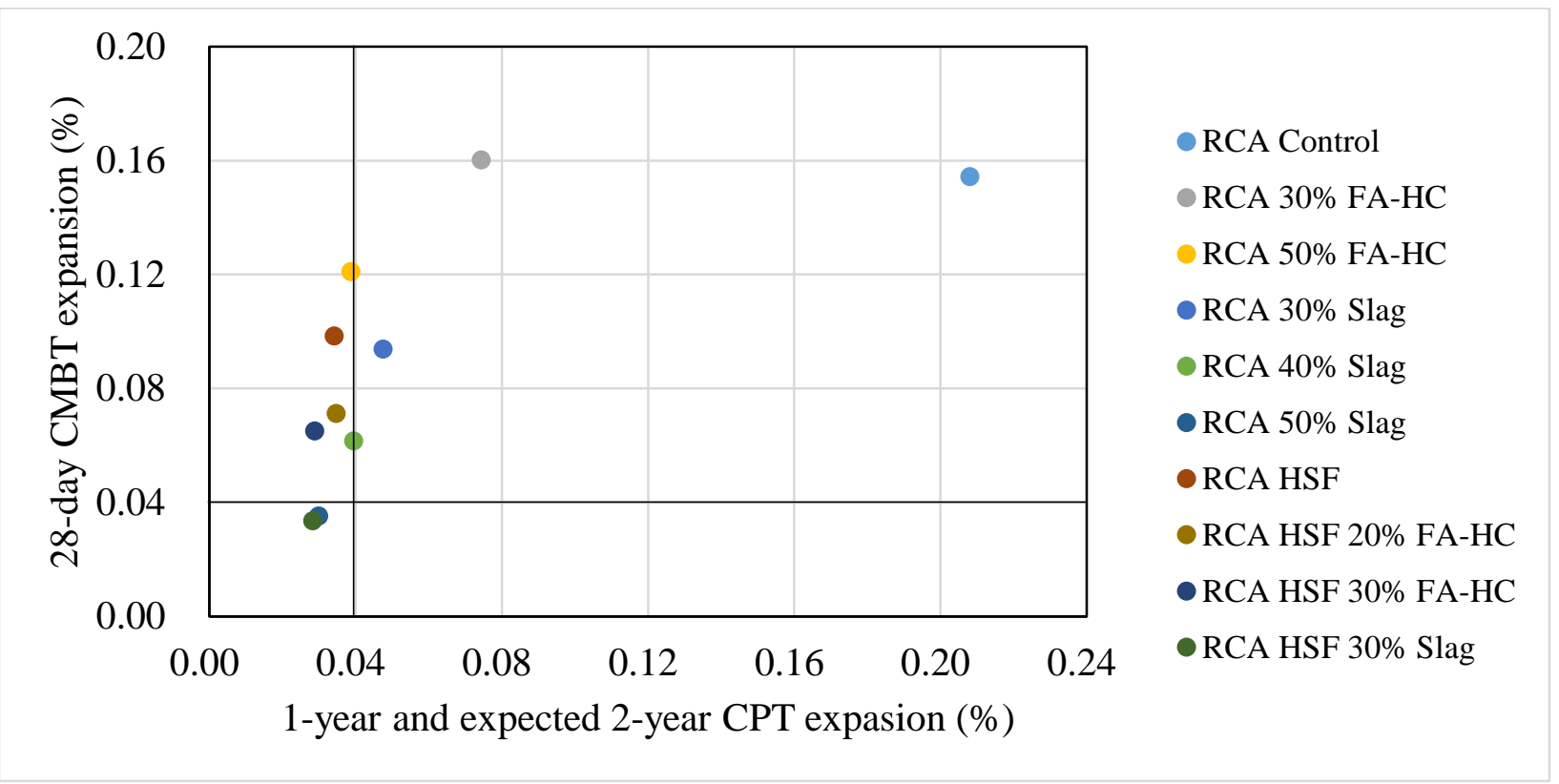

\section{Figure 4.1.36: 28-day CMBT vs. 1-year and expected 2-year CPT expansion for Sudbury RCA-M}

\subsection{Task 2}

Task 2 covers the testing of concrete prisms, concrete cylinders, extracted cores, and highway road barriers in an effort to relate the expansion obtained in the field with the expansion obtained in the lab as well as determine the efficacy of a silane-based sealer as an ASR mitigation technique. The results of each individual test and mix design will be compared with each other in order to better relate lab and field data and to better understand the difference in expansion between virgin Sudbury aggregate, Sudbury RCA, and the previously casted structure in which the RCA was obtained. The CPT procedures were used to evaluate all lab samples and the Whittemore Strain Gauge was used to measure the road barriers in the field. 


\subsubsection{Concrete Prisms}

The samples cast for task 2 include concrete prisms of two different mix designs, the first containing $420 \mathrm{~kg} / \mathrm{m} 3$ (standard mix) and the second containing $360 \mathrm{~kg} / \mathrm{m} 3$ (bridge mix). Within the two mix designs, samples were cast using three types of aggregate: virgin Sudbury aggregate, RCA from high deteriorating barriers (RCA-H), and RCA from low deteriorating barriers (RCAL). For samples of each aggregate type and each mix design, a second set of samples were cast and sealed with a silane-based sealer.

The first set of task 2 samples to be examined are the concrete prisms containing virgin Sudbury aggregate. These samples consist of prisms cast with both the standard and bridge mix, with and without the silane-based sealer. In Figure 4.2.1, the results are shown and it is clear that the samples cast with the standard mix expand at a higher rate than that of the samples cast with the bridge mix. At week 52, the samples cast with virgin Sudbury aggregate using the standard mix expanded $0.16 \%$ and the samples using the bridge mix expanded $0.14 \%$. This is due to the increased cement content of the mix design, which in turn provides more alkalis to the mix. Figure 4.2.1 shows that the silane-based sealer also reduces expansion as the standard mix reduced to $0.11 \%$ expansion at week 52 and the bridge mix reduced to $0.08 \%$ expansion at week 52 . Although all data is significantly above the CPT limit of $0.04 \%$ after 1 year, it is important to note the reduction in expansion through reducing the cement content and applying a silane-based sealer prior to undergoing testing. Additionally, the silane-based sealer was only applied prior to testing and could have a further impact if applied additionally throughout testing. 


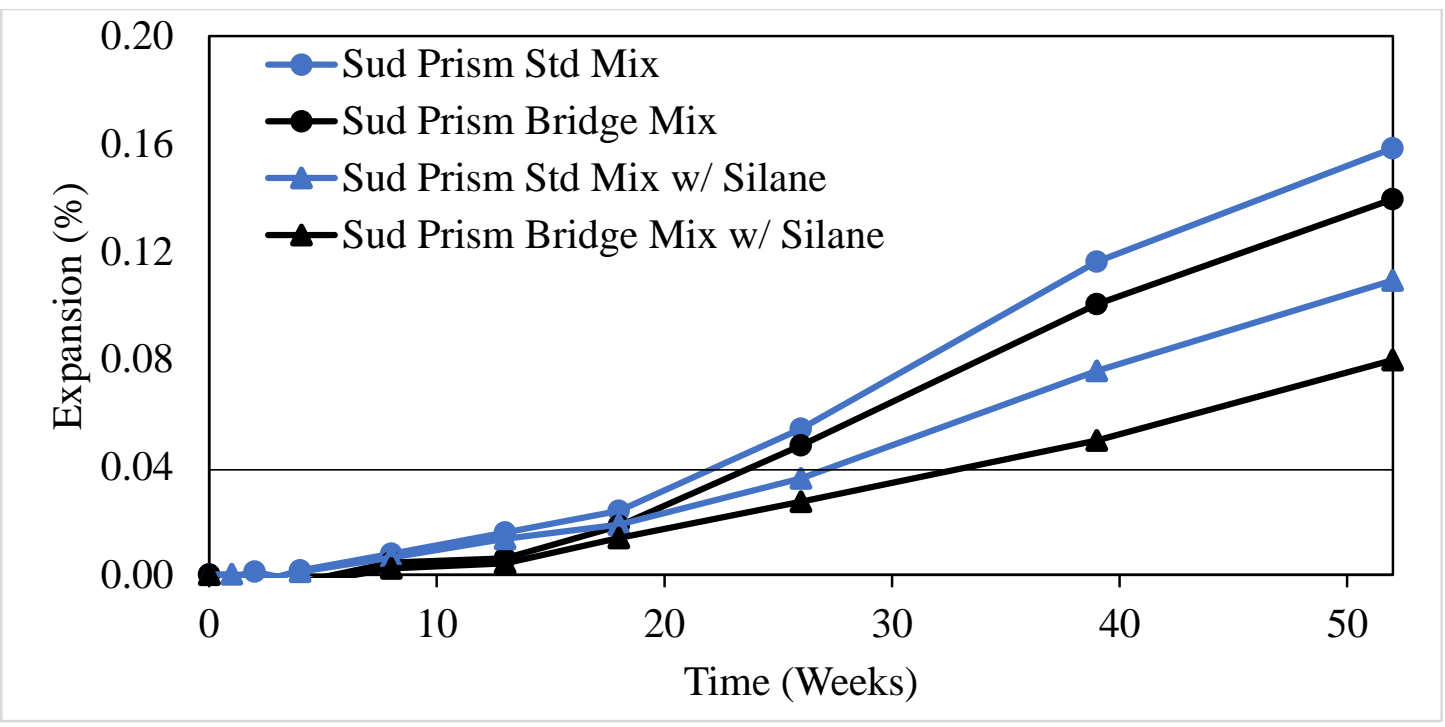

Figure 4.2.1: Prisms cast with virgin Sudbury aggregate with varying cement contents

In the next set of prisms cast for task 2 , the only change is the type of aggregate. In this set of samples, RCA-H was used. Figure 4.2.2 shows the effects of reducing the cement content and the effects of silane application on samples cast with RCA obtained from high deteriorating road barriers. Again, the standard mix produced higher expansion that that of the bridge mix, with the RCA-H standard mix prisms expanding $0.21 \%$ at week 52 and the RCA-H bridge mix prisms expanding $0.16 \%$ at week 52 . Additionally, the use of the silane-based sealer also reduced the expansion, but appears to have had less of an effect on the prisms containing RCA-H. The prisms cast with the standard mix containing RCA-H that had the sealer applied expanded $0.20 \%$ at week 52 (only a $0.01 \%$ reduction) while the bridge mix containing RCA-H with the silane-based sealer expanded $0.14 \%$ at week 52 (only a $0.02 \%$ reduction). 


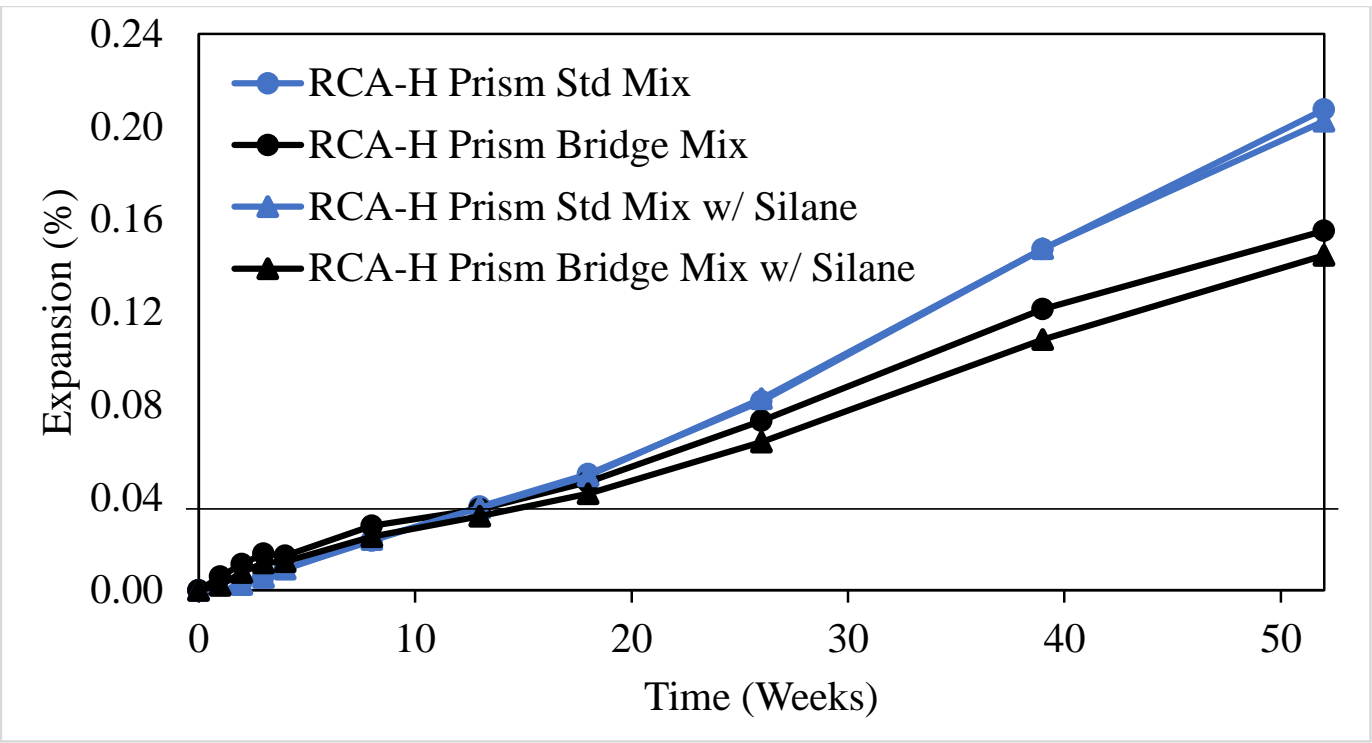

Figure 4.2.2: Prisms cast with RCA-H with varying cement contents

When comparing the same results using RCA-L as the aggregate, shown in Figure 4.2.3, similar results prevail. Again, the standard mix expands at the highest rate $(0.17 \%$ expansion at week 52$)$ while the bridge mix expands 0.15 at week 52 . In addition, the silane-based sealer also reduced the expansion of the low deteriorated prisms, with the standard mix containing the sealer expanding $0.16 \%$ at week 52 and the bridge mix containing the sealer expanding $0.10 \%$ at week 52.

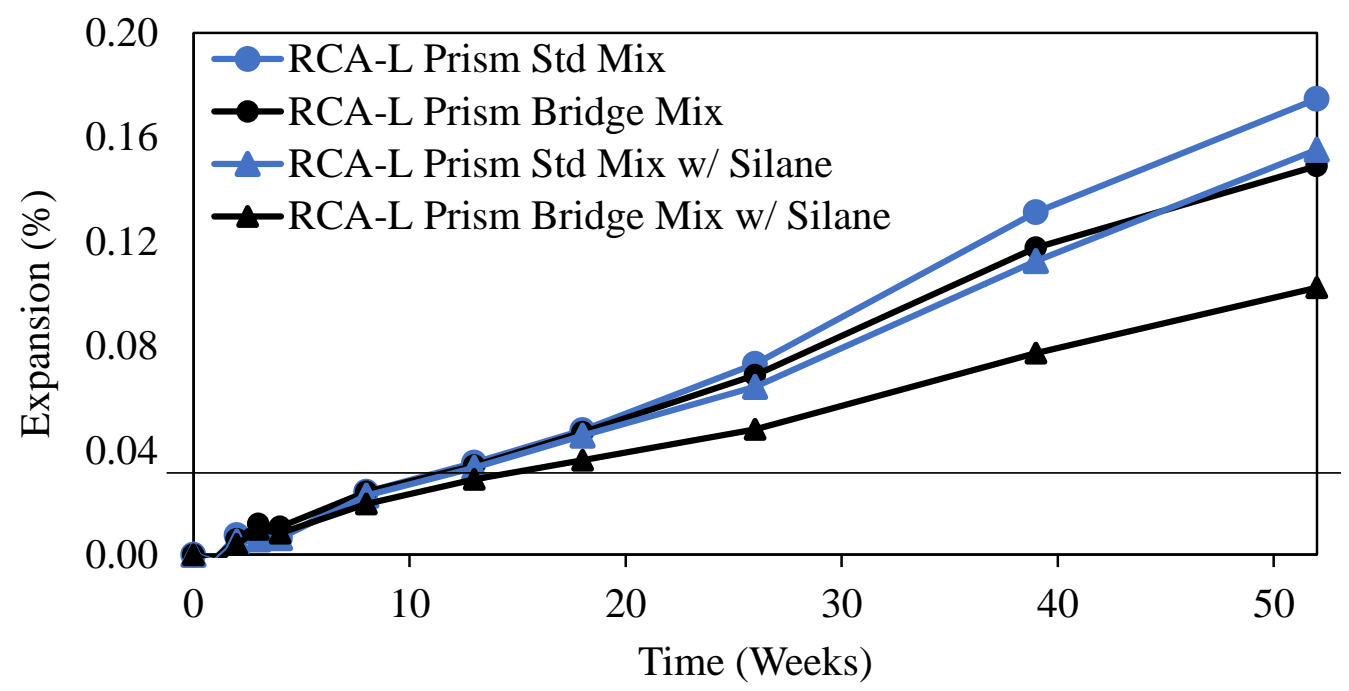

Figure 4.2.3: Prisms cast with RCA-L with varying cement contents 
Thus, in all cases, the reduction in cementing materials and the use of a silane-based sealer is effective in reducing expansion due to ASR in concrete prisms, however, all samples have exceeded the 1 year expansion limit of $0.04 \%$.

When comparing the 1-year concrete prism expansion results between the virgin Sudbury aggregate, RCA-H, and RCA-L, shown in Figure 4.2.4, it is clear that the prisms cast with virgin Sudbury aggregate expand at a lower rate than the prisms cast with RCA-H and RCA-L. The prisms cast with RCA-H expand slightly higher than the prisms cast with RCA-L, both of which show higher expansion than the virgin Sudbury mix. The difference in the expansion between each aggregate is more apparent in the standard mix than the bridge mix, which could be a result of the higher cement content magnifying the expansion results.

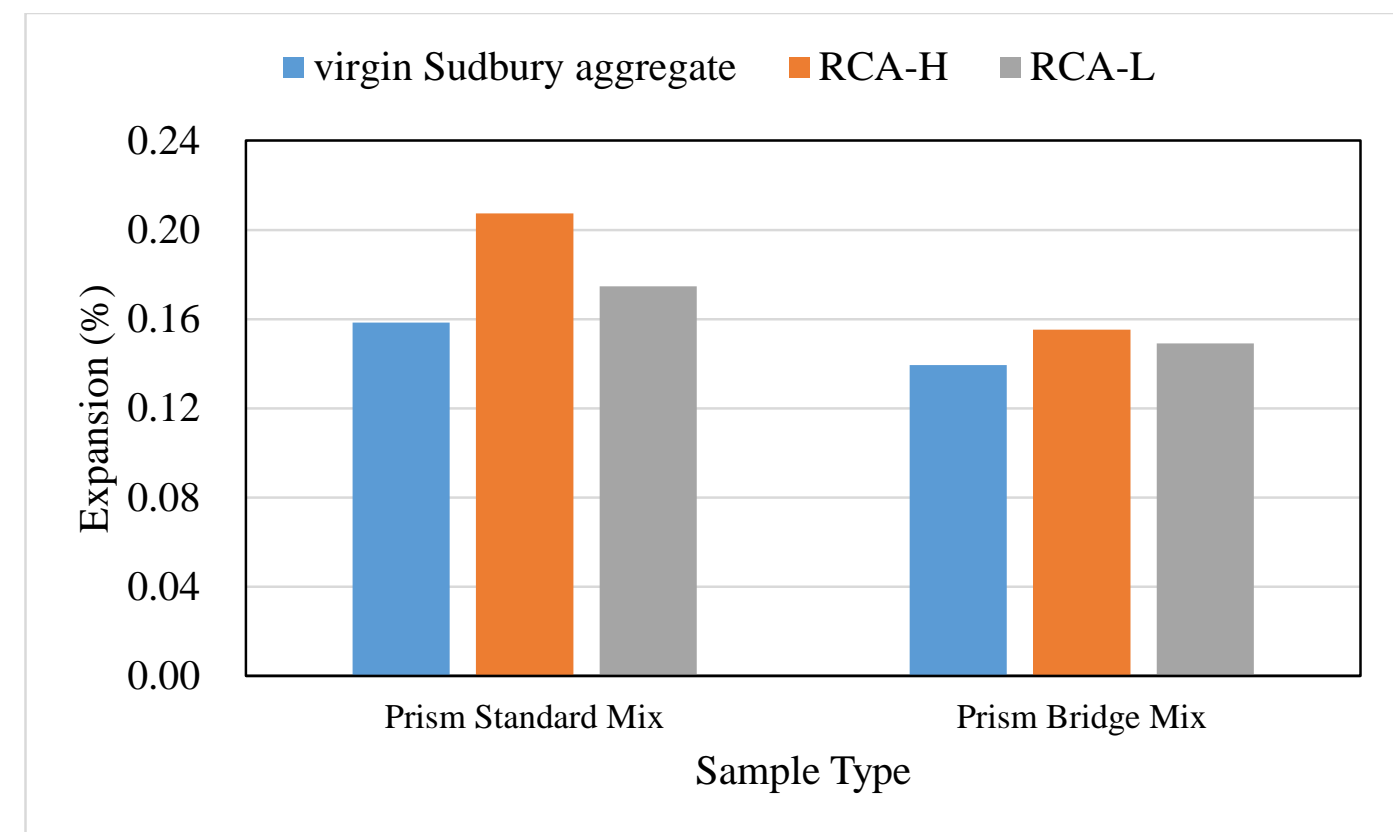

Figure 4.2.4: 52-week expansion of prisms cast with virgin Sudbury aggregate, RCA-H, and RCA-L 


\subsubsection{Concrete Cylinders}

The same samples that were cast for the concrete prisms were also cast for the concrete cylinders. It is important to note that the cylinders were measured with outside micrometers rather than the traditional length comparator used for the concrete prisms. Thus, the data obtained is not as accurate as that of the concrete prisms, as the measuring studs were flattened rather than rounded. The measuring of cylinders also allowed for more human error due to the nature of measuring using an outside micrometer by hand on flat studs. Figure 4.2 .5 shows the results for cylinders cast with virgin Sudbury aggregate. The results indicate that the cement content in the mix designs do not alter the expansion very much, as the standard mix cylinders expanded slightly less than $0.19 \%$ and the bridge mix cylinders expanded $0.19 \%$ over 52 weeks. It is clear that the silane-based sealer had a large effect on the cylinders, as the standard mix cylinders with the sealer had a 52 week expansion slightly below $0.13 \%$ and the bridge mix cylinders with the sealer had a 52 week expansion of $0.13 \%$, both reducing in about $0.06 \%$.

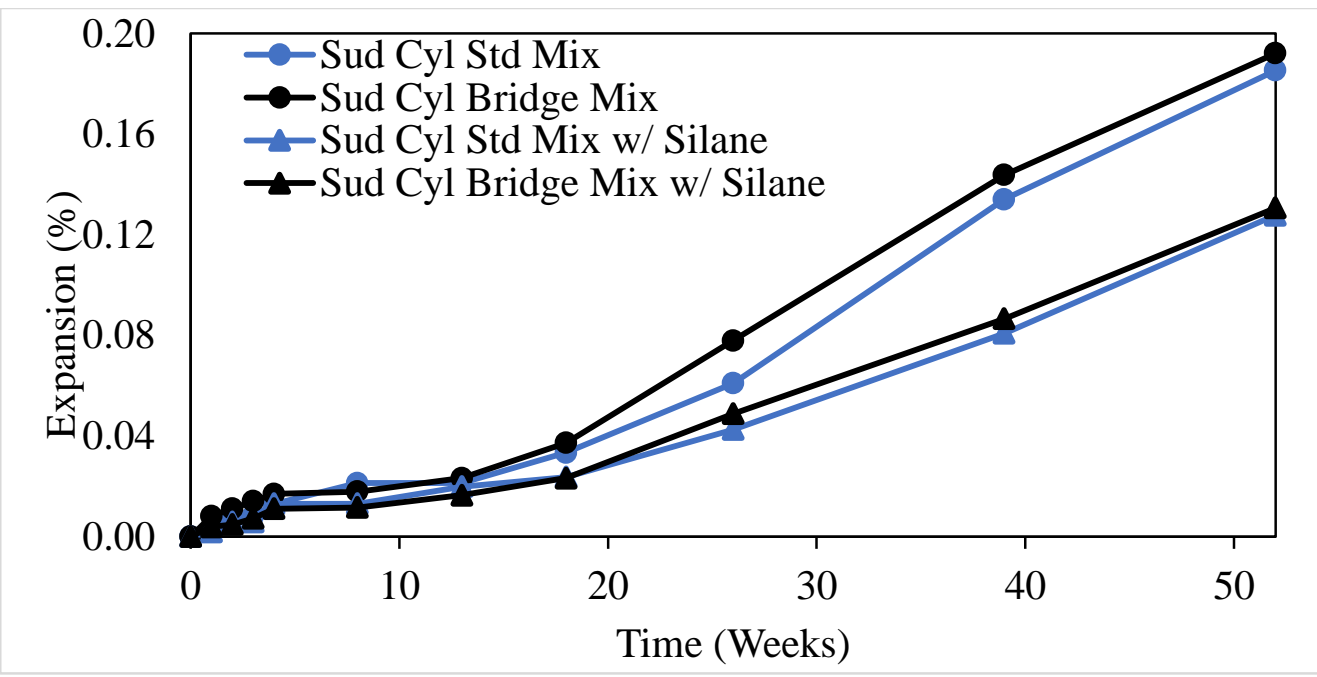

Figure 4.2.5: Cylinders cast with virgin Sudbury aggregate with varying cement contents 
When looking at the cylinder results for the samples cast with RCA-H, shown in Figure 4.2.6, the original trend found in the prisms becomes apparent. That is, that the bridge mix $(0.22 \%$ expansion at week 52) expands at a lower rate than the standard mix (slightly above $0.22 \%$ expansion at week 52), albeit a very minimal difference. The silane-based sealer continues to prove to be effective, as it reduced the expansion of the RCA-H cylinder standard mix by $0.03 \%$ and the RCA-H cylinder bridge mix by $0.04 \%$ after 52 weeks.

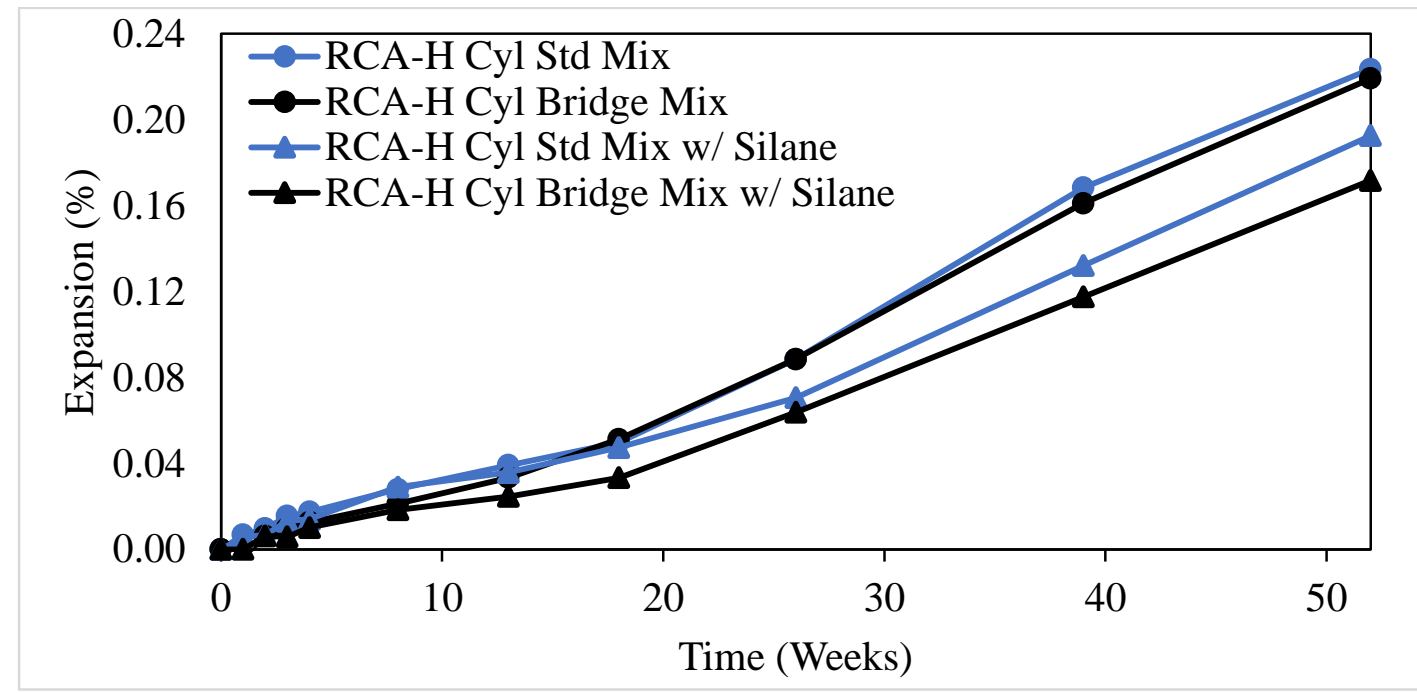

Figure 4.2.6: Cylinders cast with RCA-H with varying cement contents

Similar results prevail when looking at the cylinders cast with RCA-L, shown in Figure 4.2.7. This time around, the difference in expansion between the standard mix and bridge mix is slightly more apparent, with the standard mix expanding $0.20 \% \%$ after 52 weeks while the bridge mix expanded $0.16 \%$ after 52 weeks. The silane-based sealer mitigated some expansion, reducing the standard mix expansion by $0.06 \% \%$ and the bridge mix expansion by $0.04 \%$ through 52 weeks. 


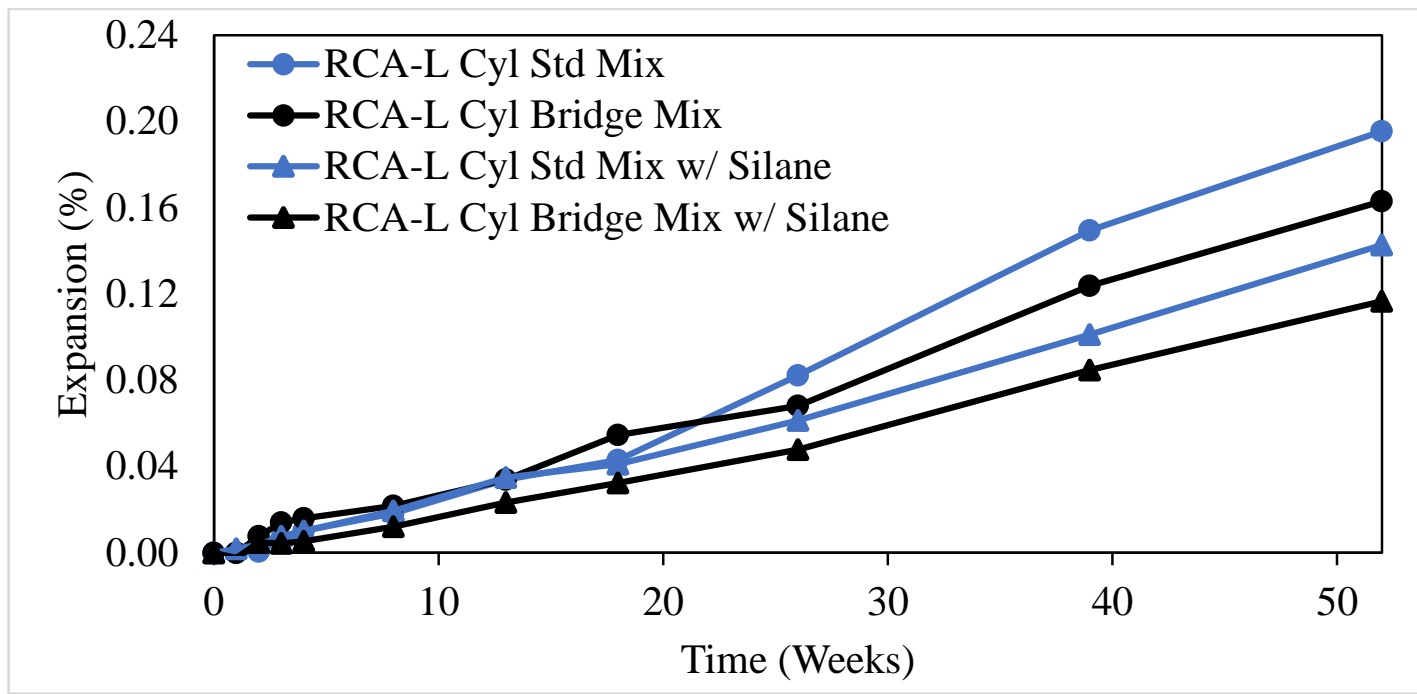

Figure 4.2.7: Cylinders cast with RCA-L with varying cement contents

The results of the cylinders indicate that by reducing the cement content in the mixture, it may or may not reduce the expansion in the cylinders. It is more likely than not that it does reduce the expansion, shown in the samples cast with RCA-L in Figure 4.2.7, because it occurred for all cases in the concrete prisms. The mixes used for the concrete prisms containing the same aggregate type is the exact same mix used for the concrete cylinders as the samples were cast together, thus the same trends should occur in both sample types. As previously mentioned, the level of difficulty to accurately measure the cylinder samples is much higher than that of the prisms, thus the expansion observed in the prisms are a more accurate representation of the true trends occurring between the different mixes.

When comparing the 1-year concrete cylinders expansion results between the virgin Sudbury aggregate, RCA-H, and RCA-L, shown in Figure 4.2.8, the same trend occurs as that of the prisms for the standard mix - RCA-H cylinders expand the most, followed closely by the RCAL cylinders, both of which are higher than the rate of expansion of the cylinders cast with virgin Sudbury aggregate. However, the cylinder cast with RCA-L show less expansion than the virgin aggregate in the bridge mix. As mentioned, the level of accuracy in measuring the cylinders are 
less than that of the prisms, thus the results obtained from the prisms should be more telling of the true trends.

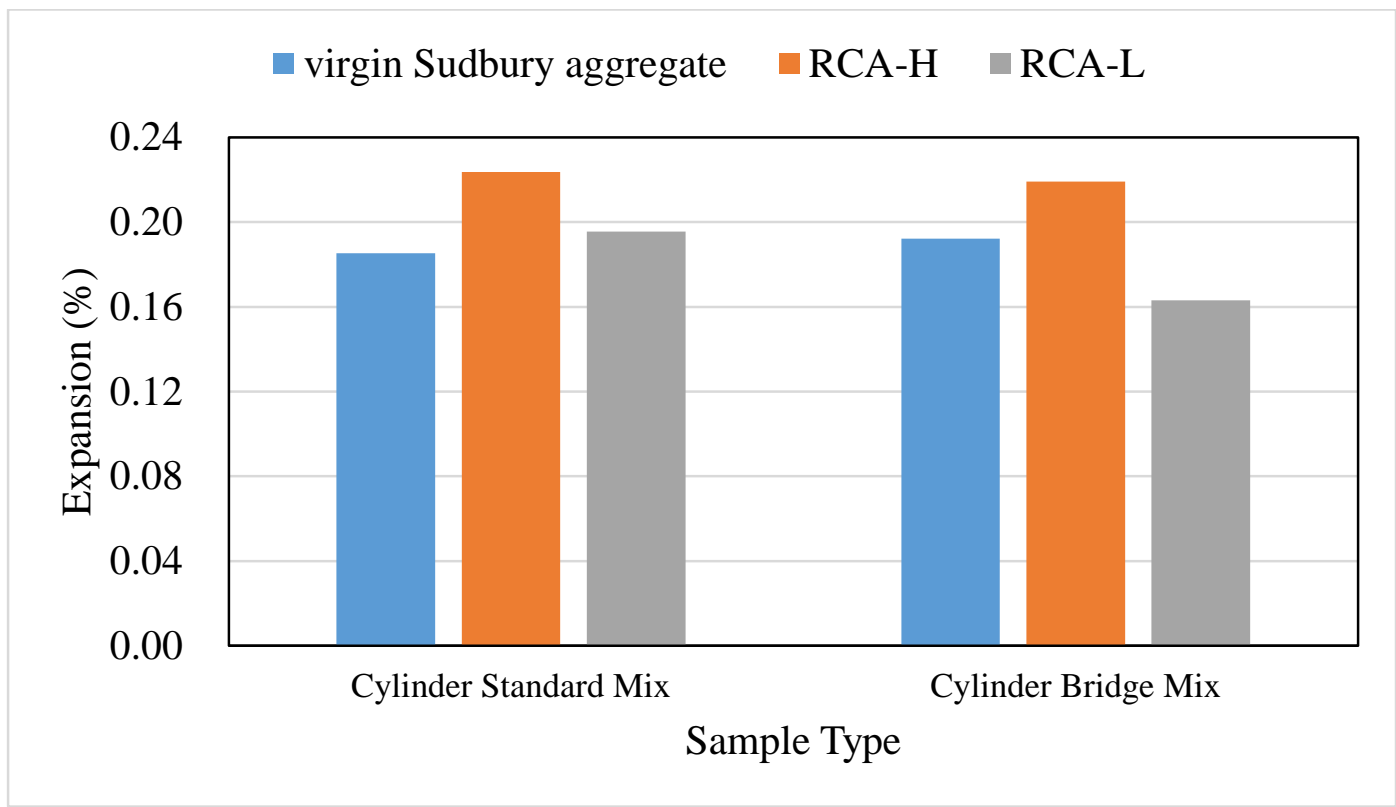

Figure 4.2.8: 52-week expansion of cylinders cast with virgin Sudbury aggregate, RCA-H, and RCA-L

\subsubsection{Extracted Cores}

The cores were extracted from the high and low deteriorating road barriers to be tested in the lab in order to observe the residual expansion that may occur. With measuring studs placed in the top and bottom of the cores, outside micrometers were used to measure the expansion, similar to that of the cylinders. Four sets of cylinders have been monitored - two sets of high deteriorated cores and two sets of low deteriorated cores, one of each set sealed with the silane-based sealer. As shown in Figure 4.2.9, the low deteriorated cores expanded $0.10 \%$ over 52 weeks, double that of the high deteriorated cores which expanded $0.05 \%$ over 52 weeks. When the silane-based sealer was applied, the expansion in the low deteriorated cores reduced by $0.02 \%$ after 52 weeks while the high deteriorated cores reduced by less than $0.01 \%$ after 52 weeks. It is clear that through the 
entire duration of testing, the sealer had a greater effect on the low deteriorated cores. This is likely due to the high deteriorated cores having surface cracks, thus being more difficult to completely coat the surface with the silane-based sealer. In addition, the expansion of both the low and high deteriorated cores seem to be reaching their peaks, suggesting that there may no longer be a sufficient alkali content to react with the aggregate.

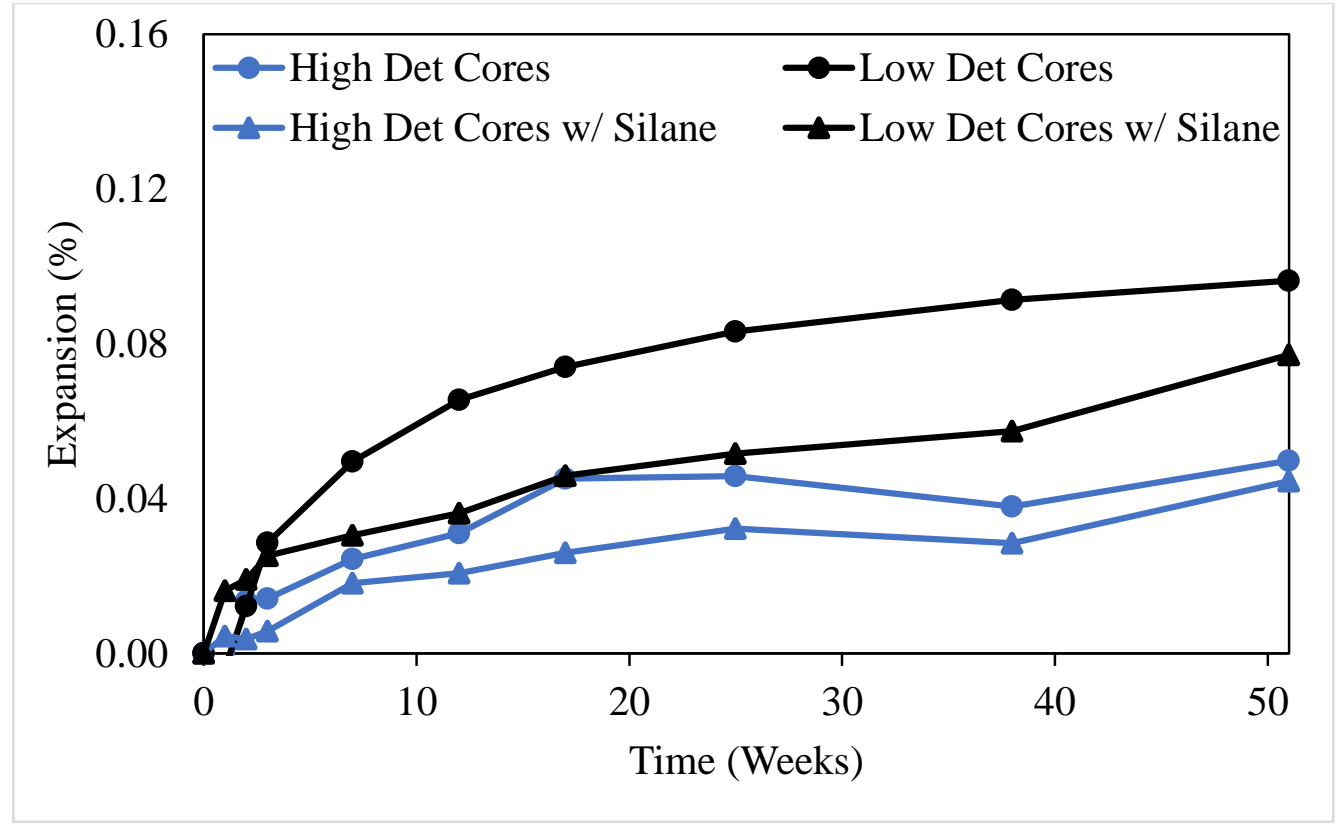

Figure 4.2.9: High and low deteriorated cores

\subsubsection{Comparing Lab Data: Prisms, Cylinders, and Cores}

When comparing the data obtained between the concrete prisms and concrete cylinders made with the standard mix, shown in Figure 4.2.10, the expansion is greater in the cylinders in all cases. The larger expansion in the cylinders is due to the increased cross-sectional area, in which decreases the leaching of alkalis from the sample. This decrease in alkali leaching causes an increase in expansion, albeit minimal, because there are more alkalis kept in the sample to react. 


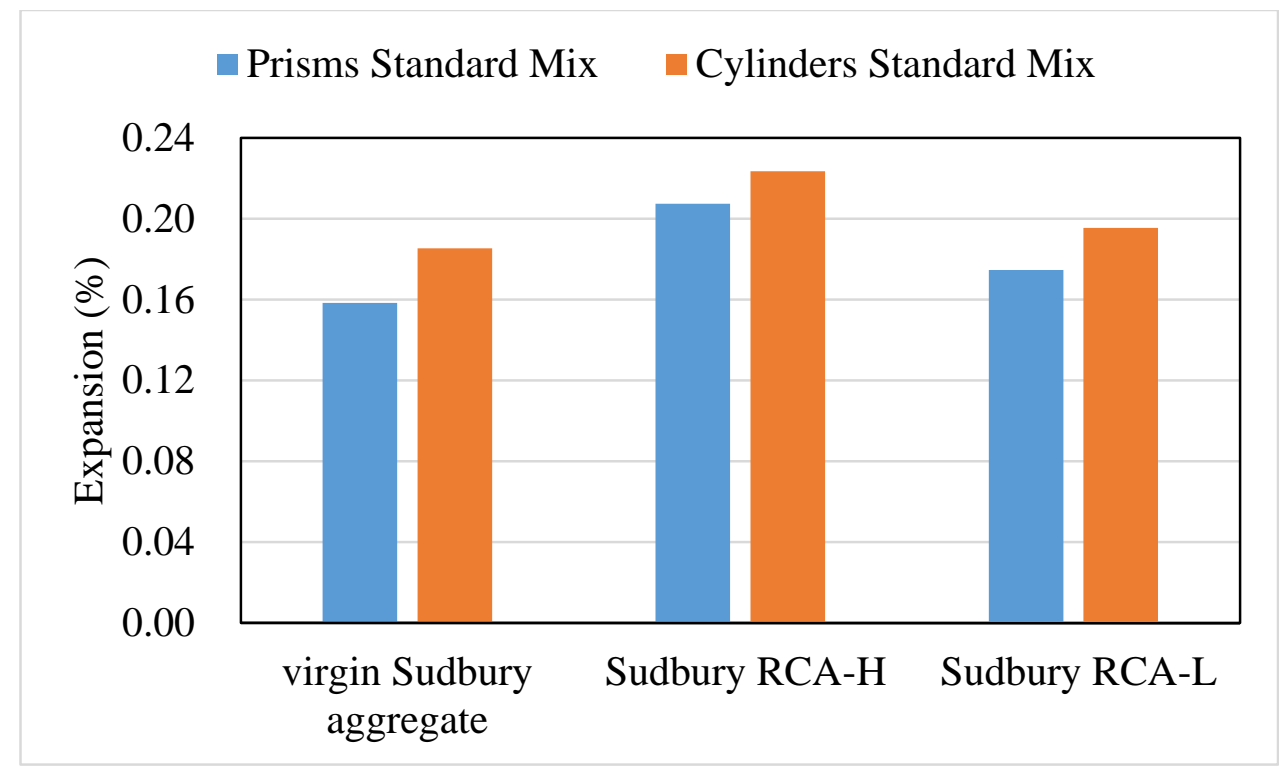

Figure 4.2.10: 52-week expansion of prisms and cylinders cast with standard mix

When looking at the effects of the silane-based sealer, shown in Figure 4.2.11, the sealer is more effective on the cylinders for all three aggregates. This is likely due to the fact that the cylinders have a smooth round surface, with minimal edges. The prisms, however, have many edges that may cause difficulty in properly coating the surface of the samples.

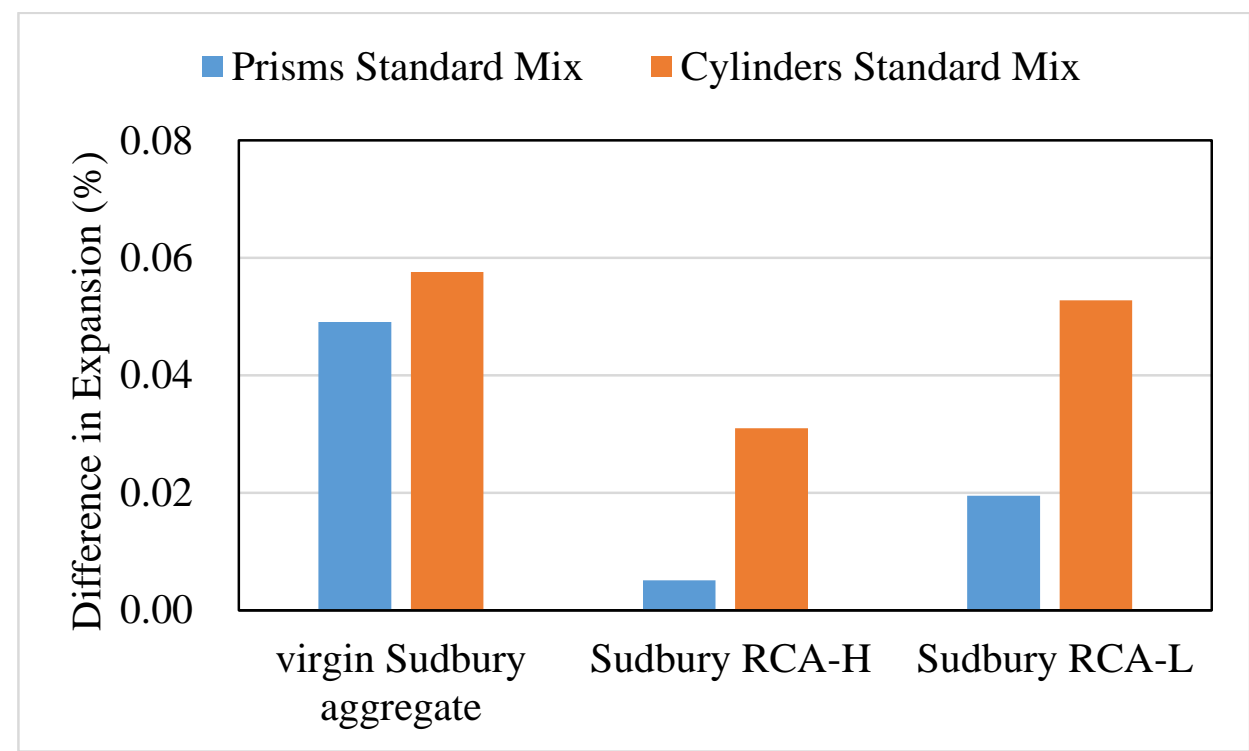

Figure 4.2.11: 52-week expansion reduction due to silane-based sealer in standard mix samples 
When comparing the expansion data between prisms and cylinders of the bridge mix, shown in Figure 4.2.12, the expansion in the cylinders is higher than the expansion in the prisms of each individual mix for all three aggregates. This further suggests that there is a reduction in alkali leaching in the cylinders, directly resulting in an increase in expansion.

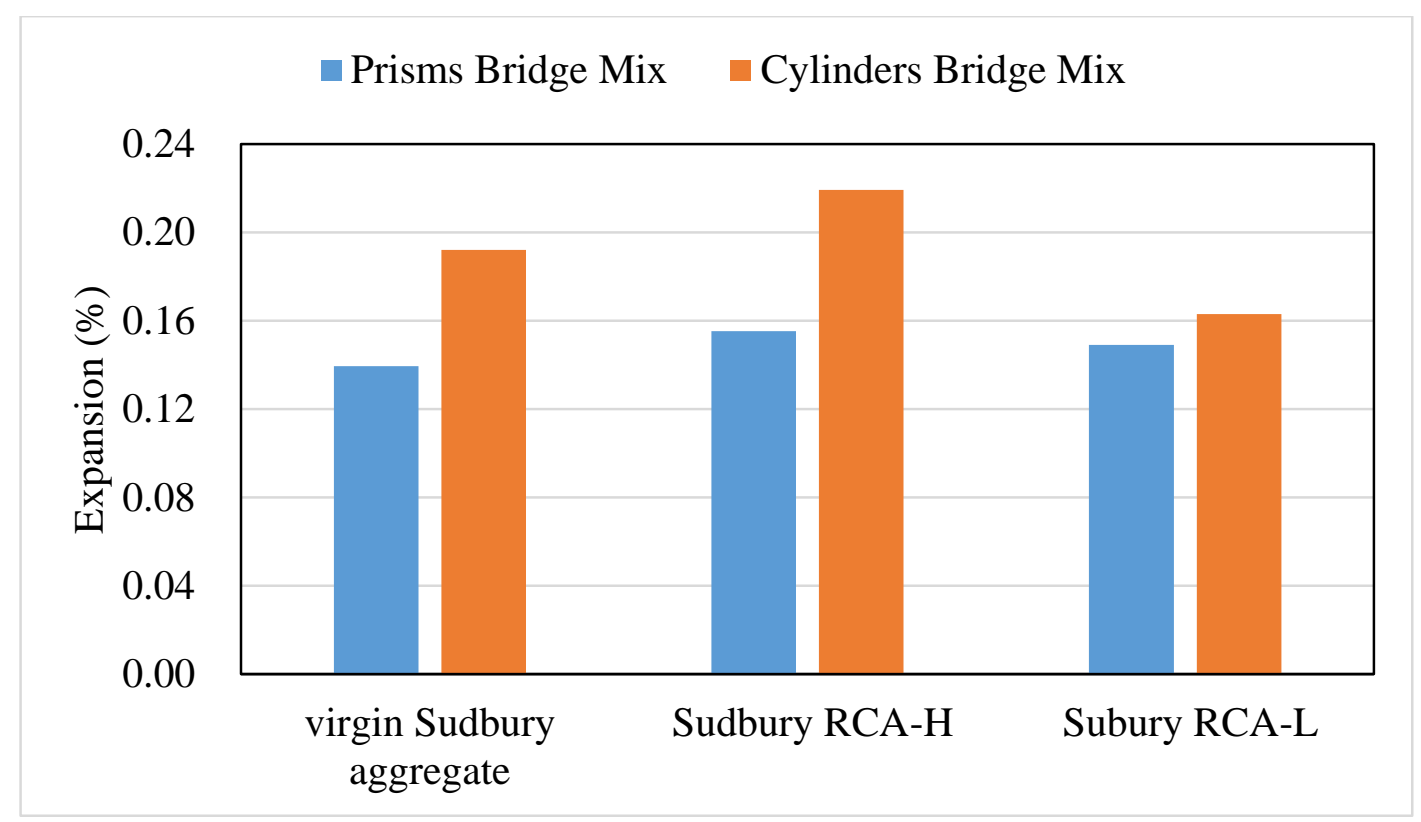

Figure 4.2.12: 52-week expansion of prisms and cylinders cast with bridge mix

It also appears that the silane-based sealer has a greater impact on cylinders made with the bridge mix samples, shown in Figure 4.2.13. Although only showing a minimal increase in reduction on the virgin Sudbury aggregate and the Sudbury RCA-L, there was a significant increase in reduction of the Sudbury RCA-H cylinders in comparison to the prisms. This further verifies the increase in reduction of the cylindrical samples that was shown in the samples cast with the standard mix. 


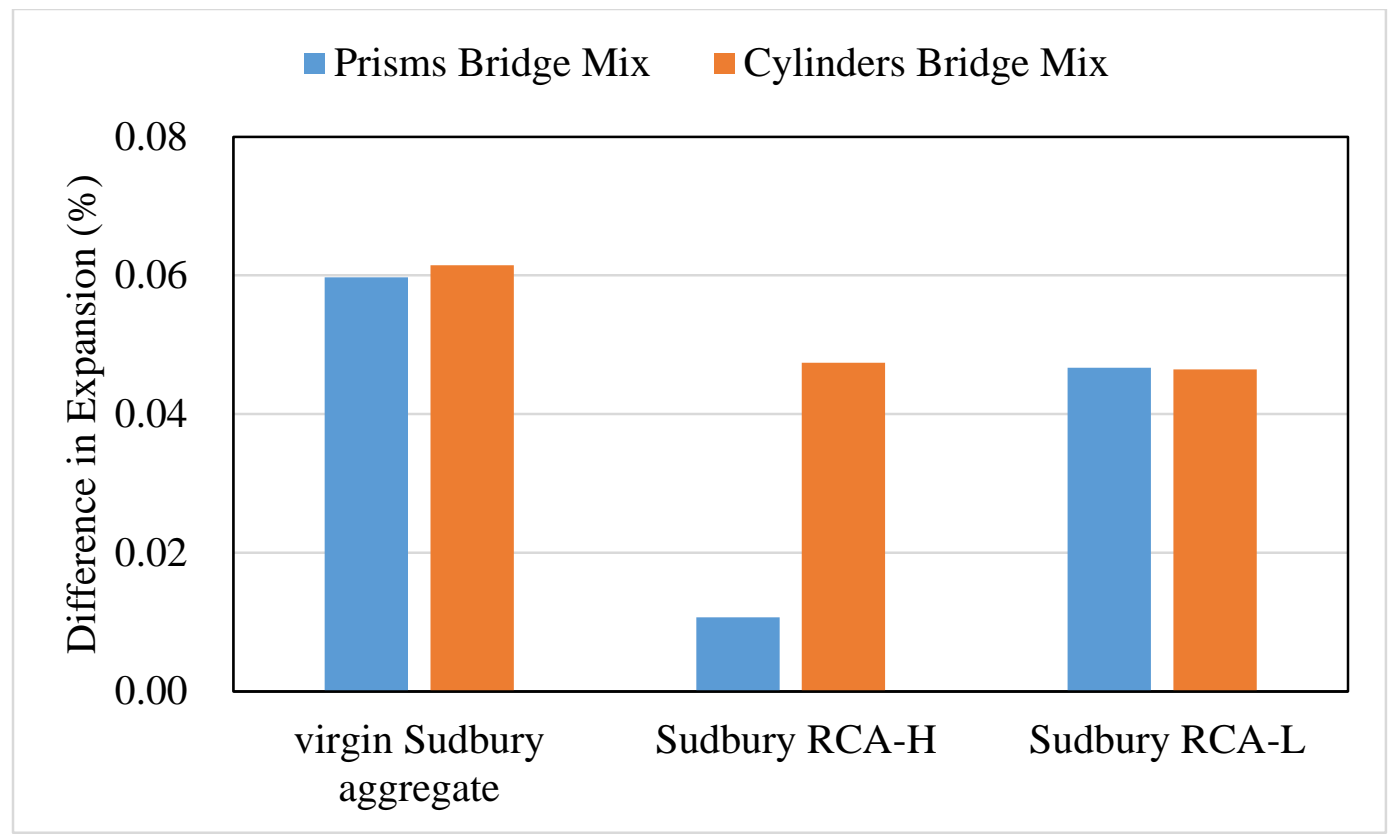

\section{Figure 4.2.13: 52-week expansion reduction due to silane-based sealer on bridge mix samples}

When comparing the expansion results between the cylinders containing RCA claimed from high and low deteriorated road barriers and the cores extracted from high and low deteriorated barriers, there is a significant increase in expansion in the casted cylinders containing RCA, shown in Figure 4.2.14. The large increase in expansion of the newly casted cylinders with RCA is due to the majority of the expansion occurring at the newly crushed faces of the aggregate. In the casted cylinders, expansion is occurring at these newly crushed faces in addition to the residual expansion occurring on the previous aggregate-cement bonds. On the other hand, the cores are only experiencing residual expansion of the original structure, which is significantly less than that of the new expansion. 


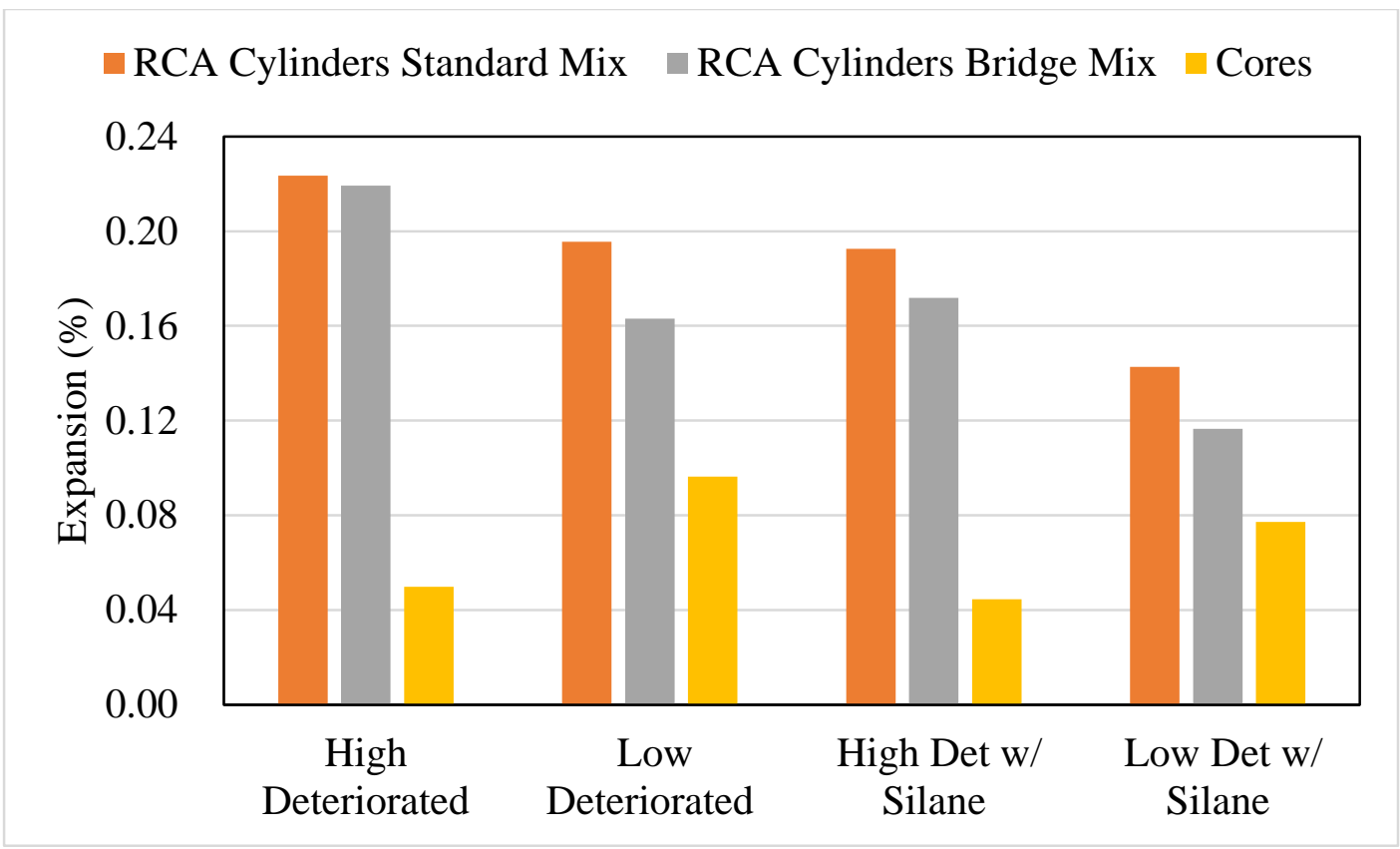

Figure 4.2.14: 52-week expansion of high and low deteriorated cylinders and cores

\subsubsection{Road Barriers}

Expansion data has been collected on the high and low deteriorating road barriers up to week 80 using the Whittemore strain gage, shown in Figure 4.2.15. The results indicate that, after 1 year, there is almost no difference in expansion between the high and low deteriorated barriers as the expansion for both sets of barriers is roughly $0.013 \%$. The measurements taken as the zero reading and at 1 year were taken at the same temperature, $1^{\circ} \mathrm{C}$, therefore thermal expansion can be considered negligible. A second reading was taken at week 80 (1 year and 6 month mark) and the expansion in the low deteriorating road barriers have slightly exceeded the expansion in the high deteriorated barriers, expanding $0.020 \%$ while the high deteriorated road barriers expanded $0.015 \%$. However, the reading taken at week 80 was taken at $22^{\circ} \mathrm{C}$, thus a correction for thermal expansion was made to obtain these values. The equation used was the linear equation for thermal expansion: 


$$
\Delta_{L}=L * \alpha * \Delta_{t} \text { where, }
$$

$\Delta_{L}$ is the thermal expansion,

$L$ is the original gauge length,

$\alpha$ is the coefficient of thermal expansion $12.2 \times 10^{6}\left({ }^{\circ} \mathrm{C}\right)$, and

$\Delta_{t}$ is the change in temperature $\left({ }^{\circ} \mathrm{C}\right)$.

It is uncertain at this point if the assumed coefficient of thermal expansion of $12.2 \times 10^{6}\left({ }^{\circ} \mathrm{C}\right)$ is accurate, thus further measurements shall be taken around $1^{\circ} \mathrm{C}$ in order to determine an accurate coefficient.

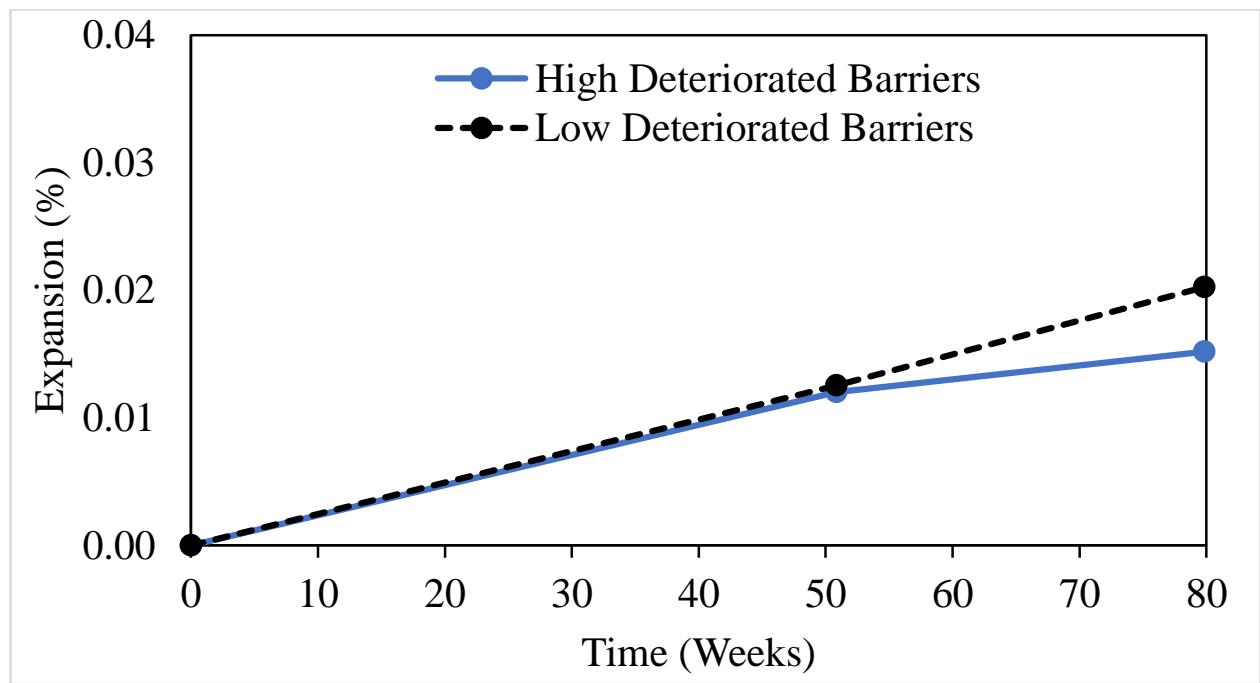

Figure 4.2.15: 80 week expansion data for high and low deteriorated road barriers

\subsubsection{Comparing Lab and Field Data}

The expansion data obtained on the extracted cores undergoing ASR testing through the CPT can be compared to the road barriers undergoing the effects of ASR and freeze-thaw deterioration in the field, shown in Figure 4.2.16. As expected, the expansion measured through one year on the barriers are very minimal compared to the lab samples, because they are not exposed to severe (accelerated) conditions. The barriers also contain restrictions to expansion due to the surrounding concrete around the measuring studs. The combined effects of ASR and freeze-thaw deterioration in the field is still minimal in comparison to the accelerated ASR testing in the lab through the 
concrete prism test. The barriers will continue to be monitored for years to come in an attempt to draw a correlation between lab and field data for structures affected by ASR.

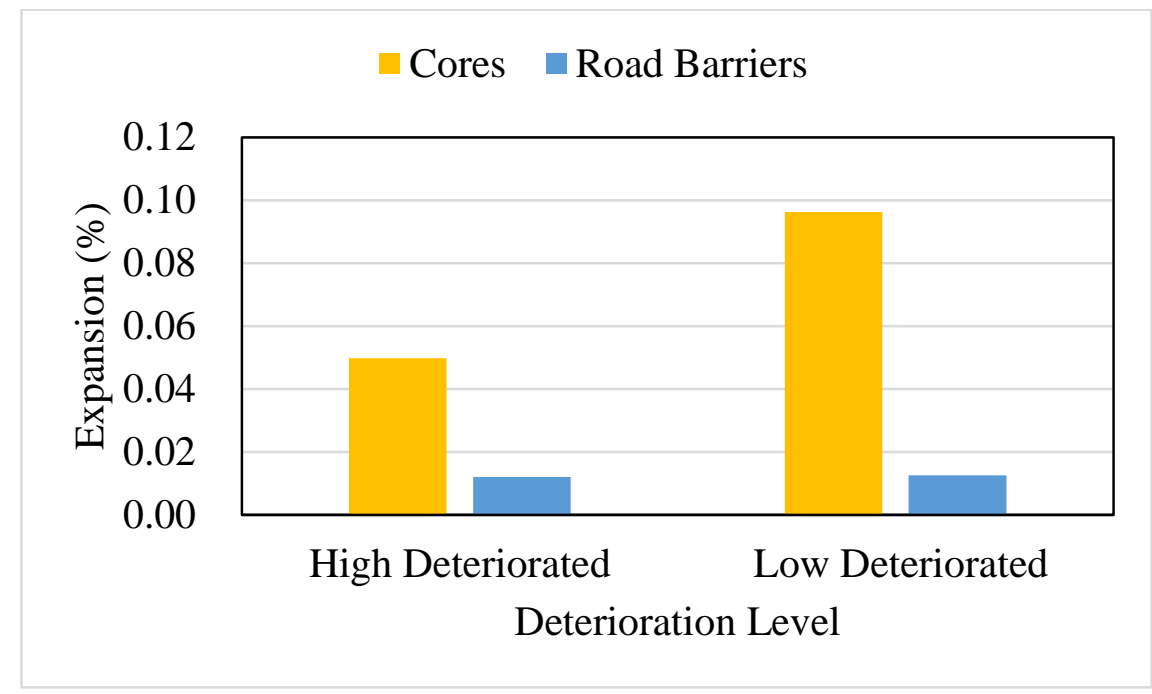

Figure 4.2.16: 1-year expansion data of high and low deteriorated cores and road barriers

\subsection{Comparing Task 1 and Task 2 Data}

The data obtained in task 1 (mortar bars, microbars, and prisms) can be correlated to the data obtained in task 2 (prisms, cylinders, cores, and road barriers). A comparison can be made between the ability of the SCM used in task 1 and the silane-based sealer used in task 2 in mitigating expansion in concrete due to ASR. In addition, a comparison can be made between the difference in expansion between RCA-M, used in task 1, and RCA-H and RCA-L, used in task 2. It has already been shown that there are minimal differences in the RCA obtained from high and low deteriorated road barriers used in task 2 , so it will now be compared to the mixed RCA used in task 1 . The only samples that contain the same mix design and specimen shape from task 2 are the concrete prism standard mixes containing virgin Sudbury aggregate, Sudbury RCA-L, and Sudbury RCA-H from task 2. Thus, these samples will be used to compare to the concrete prisms results in task 1 . 


\subsubsection{Comparing RCA-M, RCA-H, and RCA-L}

The difference in expansion between RCA-H and RCA-L from task 2 has already proven to be minimal, shown in Figure 4.2.4. A comparison can be seen of the RCA-M used in task 1 to that of the RCA-H and RCA-L used in task 2 in Figure 4.2.17. The figure shows that the RCA-M control mix from task 1 has an identical 1-year expansion value of $0.21 \%$ as the RCA-H standard mix (which is an identical mix design), both of which are very similar to the expansion measure for the RCA-L standard mix. $(0.17 \%)$ Thus, it can be concluded that the three RCA's containing different levels of deterioration are interchangeable.

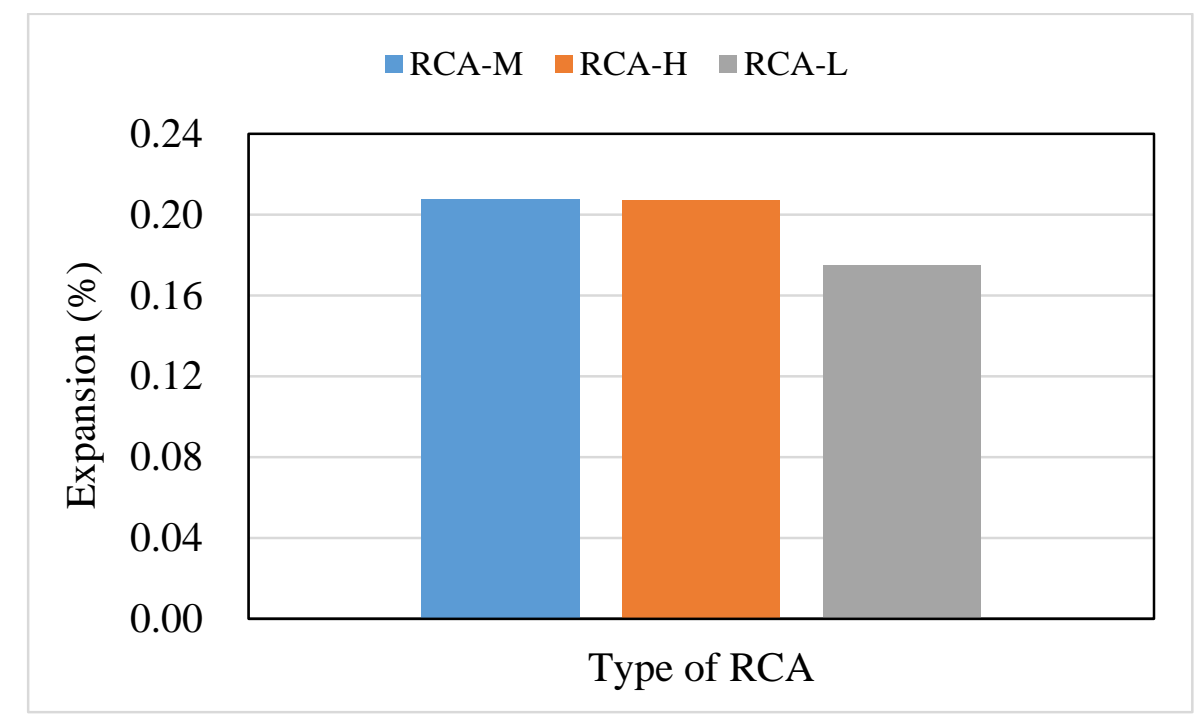

Figure 4.2.17: Comparing 1-year expansion results of RCA-M, RCA-H, and RCA-L

\subsubsection{Comparing the Effects of SCM and the Silane-based Sealer}

When looking at the results of the silane-based sealer on reducing the expansion in concrete due to ASR in task 2, it is clear that a reduction in expansion does occur. That being said, compared to the results obtained through the use of SCM in task 1, the reduction in expansion through the sealer is minimal, shown in Figure 4.2.18. When looking at the expansion data of the prisms in task 1, the samples containing SCM that experienced the most expansion that were cast with virgin 
aggregate are the samples containing $30 \%$ FA-HC, with a 1-year expansion of $0.037 \%$, compared to the control mix of $0.168 \%$ expansion at 1 year. For the samples cast with Sudbury RCA-M containing SCM, the least effective mix contained 25\% FA HA-LC, with a 1-year expansion of $0.049 \%$ compared to the control mix of $0.208 \%$ expansion at 1 year. This indicates that mix containing the least effective SCM for virgin Sudbury aggregate reduced the expansion by $0.145 \%$ while the least effective SCM for RCA-M reduced the expansion by $0.172 \%$. In comparison, the silane-based sealer was most effective on the virgin Sudbury aggregate samples, reducing the expansion by only $0.049 \%$. This suggests that the SCM are at least 3 times more effective than the silane-based sealer at reducing expansion due to ASR in new concrete when only 2 applications of the sealer are applied prior to testing. It should be noted that the main use of silane would be to mitigate rate of expansion in existing structures suffering ASR, where SCM cannot be used. For new mixtures, SCM is the right preventive measures. In situations where minimal expansion is required, the use of SCM in the mixtures with regular treatment of the structure by silane might provide the safest level of ASR prevention.

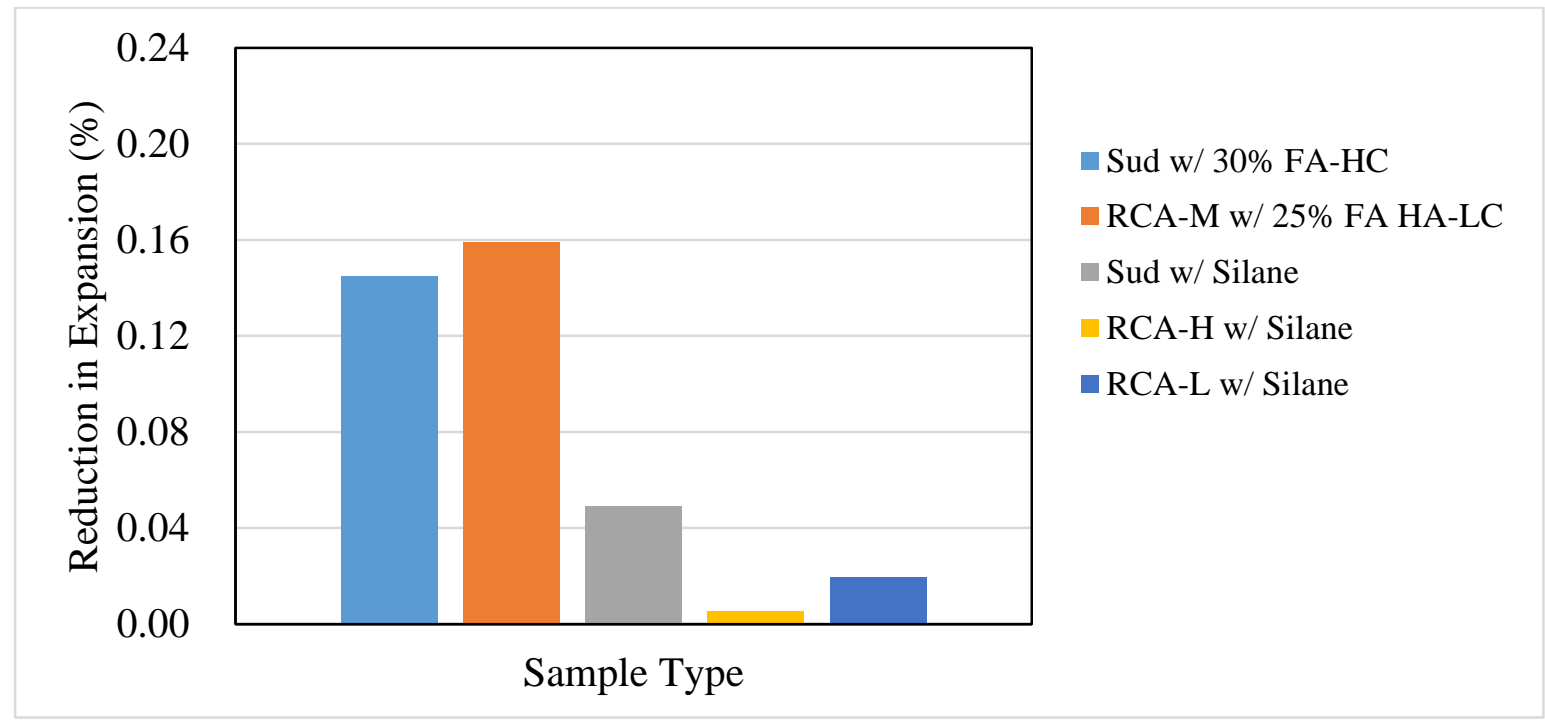

Figure 4.2.18: Comparing the effects of SCM and the silane-based sealer as an ASR mitigating technique 


\section{Chapter 5: Discussion}

\subsection{Analyzing the Effects of SCM on Sudbury Aggregates}

When comparing the effects of different SCM, the results were consistent for both aggregate types and all test methods. The most effective SCM was silica fume, found in the HSF blended cement. The FA LA-LC (used in the AMBT and CPT) was the next most effective, followed by the slag, FA HA-LC (used in the CPT), and finally the FA-HC. There is no doubt that the silica fume was the most effective SCM, as the HSF blended cement only contains $8 \%$ silica fume and was enough to reduce the expected 2-year expansion of virgin Sudbury aggregate to $0.027 \%$ and RCA to 0.034\%. When the HSF blended cement was used in addition to another SCM, all 4 samples contained results that are expected to pass the $0.04 \%$ 2-year limit through linear extrapolation. Linear extrapolation of the slope was completed on the last two data points (week 52 and week 65) in order to estimate the 2-year data. In addition, all samples containing HSF passed the AMBT and only 2 of 8 samples containing HSF along with another SCM failed the CMBT test. The effectiveness of the silica fume is attested to its ability to reduce the calcium hydroxide content of concrete (Siddique and Khan, 2006). It is important to note that only samples with $8 \%$ silica fume were tested, thus the effects of a lesser or higher dosage are unknown. In addition, the same dosage may not be as effective for other aggregates.

The low-calcium fly ash used in the AMBT and CMBT was the next most effective SCM, as using just 25\% FA LA-LC caused the virgin Sudbury aggregate to pass both tests and the Sudbury RCA to pass the AMBT. When used in combination with the HSF blended cement, all samples passed both the AMBT and CMBT. The CPT samples contained FA HA-LC, which contained 3.68\% $\mathrm{Na}_{2} \mathrm{O}_{\text {e }}$ produced higher expansion results, compared to FA LA-LC used in the 
AMBT and CPT which contained an alkali content of $1.86 \%$. Fly ash's ability to reduce expansion due to ASR is due to its ability to react with alkalis present in the concrete, reducing the available alkalis to react with the aggregate to form ASR (Siddique and Khan, 2006). When a fly ash has a higher alkali content, it becomes more difficult for the fly ash to reduce expansion. This is because the fly ash is also increasing the alkali content of the concrete, creating more work for the SCM. In addition, a higher alkali content in the concrete directly relates to a higher expansion. Figure 5.1.1 (Shehata and Thomas 2010) shows the effects of the alkali content $\left(\mathrm{Na}_{2} \mathrm{O}_{e}\right)$ on the expansion of Sudbury, Spratt, and Jobe aggregates. Looking at the Sudbury curve, there is a large increase in expansion when moving from a $0.8 \% \mathrm{Na}_{2} \mathrm{O}_{\text {e }}$ content to a $0.9 \% \mathrm{Na}_{2} \mathrm{O}_{\text {e }}$ content. Thus, the increased alkalis provided from the fly ash has a large impact on the increased expansion of the samples.

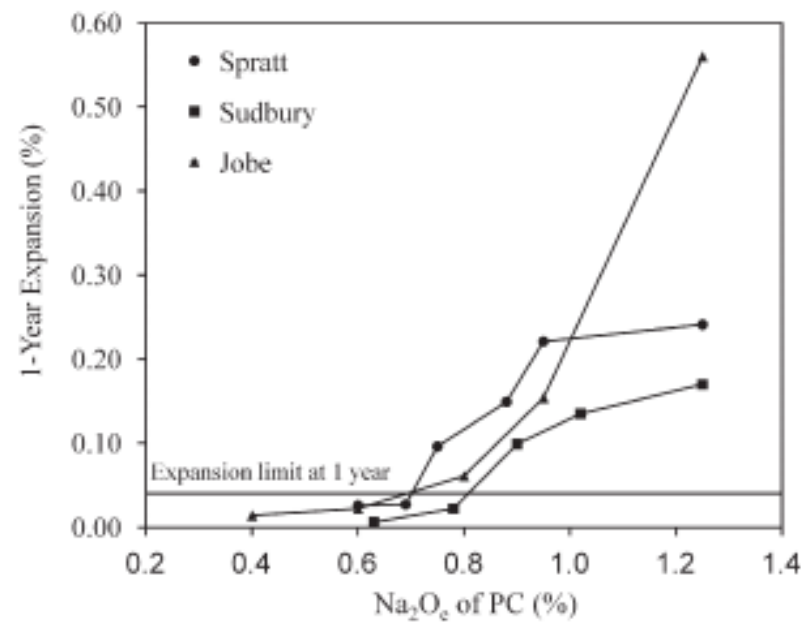

Figure 5.1.1: Effects of Portland cement alkali content on expansion of concrete prisms (Shehata and Thomas, 2010)

The slag was the third most effective SCM, in which 50\% slag was required to mitigate expansion to pass the AMBT and CMBT for both aggregate types. For the CPT, $30 \%$ slog was required to get an expected 2-year expansion below $0.04 \%$ for the virgin aggregate, while $40 \%$ slag was required for the RCA. Slag, much like fly ash, is able to reduce the alkalinity of concrete 
due to its ability to bond with available alkalis in the structure in addition to reducing the mobility of the alkalis (Siddique and Khan, 2006). Finally, the least effective SCM was the FA-HC, as using up to $50 \%$ FA-HC did not reduce the expansion enough to pass the AMBT or CMBT for either aggregate and was the only SCM to do so. This is due to the large amount of calcium oxide present $(26.47 \%)$ in the high-calcium fly ash, compared to the $3.67 \%$ in the FA LA-LC. A high-calcium fly ash is not as effective because (1) it does not consume $\mathrm{Ca}(\mathrm{OH})_{2}$ as effectively as low-calcium fly ash and (2) it produces $\mathrm{CSH}$ at a high calcium-silica ratio which does not bind alkalis as effectively as the CSH in low-calcium fly ash, which contains a low calcium-silica ratio (Shehata and Thomas, 2000). Therefore, as the $\mathrm{CaO}$ content in fly ash increases, it becomes less effective in in reducing the pore solution alkalinity resulting in a greater proportion of alkalis to be available for reaction (Shehata and Thomas, 2000).

\subsection{Analyzing the Expansions of Virgin Sudbury aggregate and Sudbury RCA-M}

The difference in expansion between samples containing Sudbury RCA-M and samples containing virgin Sudbury aggregate have been rather common between the concrete microbar test and the concrete prism test. These two tests have shown that the Sudbury RCA-M consistently expanded at a higher rate than the virgin Sudbury aggregate, suggesting larger quantities of SCM are required to mitigate ASR in Sudbury RCA than the virgin aggregate, shown in Figures 4.1.21 and 4.1.31. The reason for this is likely the contribution of alkalis from the residual mortar, expansion of existing ASR gel, or the exposure of new faces on the existing aggregate due to crushing (Shehata et al. 2010). The results obtained through the accelerated mortar bar test do not coincide with this trend, as the expansion differences between the two types of aggregate were sporadic. Unlike the CMBT and CPT, in which all samples containing RCA exceeded its counterpart sample containing virgin aggregate, 4 of the virgin aggregate samples expanded more than the RCA samples at 14 
days, shown in Figure 4.1.9. It is believed that this is due to two main reasons that are caused by the crushing of the aggregate. The first reason being that when crushing RCA, the cement is the first to break because it is far weaker than the original aggregate. Because of this, the majority of the finer sizes of the crushed RCA (retained on \#30, \#50, \#100 sieves) are likely to be original cement whereas the majority of the larger sizes (retained on \#8 and \#16 sieves) are likely to be original aggregate. This causes a bit of an inaccuracy in the AMBT test because smaller particles contain larger surface area, thus the cement particles, which will not contribute to ASR expansion, take up more surface area within the sample. As a result, there is more reactive materials in the virgin samples than the RCA samples and may be more pronounced due to the smaller aggregate sizes. The second, and likely the more predominant reason, is that in crushing the aggregate to such small sizes for the AMBT, the majority of the previous bonds between cement paste and aggregate that occurred in the original structure are now separated, which is not the case in the aggregate used for the CMBT and CPT, as the aggregate size is much larger. That is, in breaking up the aggregate to meet the gradation requirements for the AMBT, the old gel is broken down and expansion of the old gel will no longer occur because the gel is lost during crushing. Since expansion is only occurring on the newly created bonds of aggregate and cement, there is little to no residual expansion occurring in the AMBT samples. The breakdown and loss of the gel is likely more apparent in Sudbury aggregate due to the fact that the gel formation occurs around the aggregate, rather than within the aggregate such as some other aggregate types. On the other hand, the CMBT and CPT RCA samples contain both the previous bonds and the new bonds, thus the residual expansion is causing an increase in expansion of the RCA samples in comparison to the virgin aggregate samples. The effects of residual expansion were proven by the expansion that 
occurred in the extracted cores in task 2 , as both high and low deteriorated cores undergoing the CPT for 1 year experienced residual expansion, shown in Figure 4.2.14.

Although the AMBT samples containing RCA were prepared and cast in accordance to Adams et al. (2013), it seems that the AMBT might not be as effective as other tests in determining reactivity of RCA and efficacy of preventive measures. When crushing aggregate to fine aggregate sizes, the previous aggregate-cement interface are being broken up. In doing so, the residual expansion will not be as aggressive as it would be in samples containing coarse aggregate like the CMBT and CPT. Since the purpose of testing this aggregate is to test its usability as a coarse aggregate, the CMBT and CPT results are a more indicative of what is expected to occur in a structure.

\subsection{Comparing CPT Results of Sudbury Aggregates to Minimum SCM Requirements}

Given the CPT results obtained of the control mixes for both the virgin Sudbury aggregate $(0.168 \%$ after 1 year) and Sudbury RCA (0.208\% after 1 year), both aggregates can be classified as highly reactive aggregates as per Table 2 from CSA A23.2-09 27A (2014), although this type of aggregate has been classified as moderately reactive aggregates in the past. If we assume that the virgin or RCA investigated here will be used in bridge barriers exposed to water and humidity, the degree of reactivity can be classified as Level 4 (for highly reactive aggregate, which is the case based on the expansion obtained here) as per Table 3 from CSA A23.2-09 27A (2014). From Tables 4 and 5 of CSA A23.2-09 27A (2014), an assumption was made that the bridge was intended to have a service life between 5 and 75 years, thus strong preventative action is required. From Table 7 of CSA A23.2-09 27A (2014), the minimum level of SCM was determined for the mentioned circumstances, shown in Table 5.3.1. When using a combination of SCM, the standard requires 
that the sum of the parts used to equal 1. For example, if a third of the required silica fume is used, a minimum of two-thirds of the required fly ash must be used.

Table 5.3.1: Minimum level of SCM required for Sudbury aggregates

\begin{tabular}{|c|c|c|c|}
\hline Type of SCM & $\begin{array}{c}\text { Total alkali } \\
\text { content of SCM } \\
\mathrm{Na}_{2} \mathrm{O}_{\mathrm{e}}(\%)\end{array}$ & $\begin{array}{l}\text { Chemical } \\
\text { composition } \\
\text { requirement } \\
\text { (\% oxides) }\end{array}$ & $\begin{array}{c}\text { Minimum Level } \\
\text { of SCM }(\%)\end{array}$ \\
\hline \multirow[t]{3}{*}{ Fly Ash } & $<3.0$ & $\mathrm{CaO}<8 \%$ & 25 \\
\hline & & $\begin{array}{c}\mathrm{CaO}=8 \%- \\
20 \%\end{array}$ & 30 \\
\hline & & $\mathrm{CaO}>20 \%$ & $\begin{array}{c}\text { Testing required } \\
\text { as per CSA } \\
\text { A23.2-09 28A }\end{array}$ \\
\hline \multirow[t]{3}{*}{ Fly Ash } & $3.0-4.5$ & $\mathrm{CaO}<8 \%$ & 30 \\
\hline & & $\begin{array}{c}\mathrm{CaO}=8 \%- \\
20 \%\end{array}$ & 35 \\
\hline & & $\mathrm{CaO}>20 \%$ & $\begin{array}{c}\text { Testing required } \\
\text { as per CSA } \\
\text { A23.2-09 28A }\end{array}$ \\
\hline Slag & $<1.0$ & & 50 \\
\hline Silica Fume & $<1.0$ & & $10.7 *$ \\
\hline
\end{tabular}

The low-calcium fly ash used in the concrete prisms, labelled FA HA-LC, had a 7.24\% CaO content along with a $3.68 \%$ alkali content, as shown in Table 3.2.2, thus requiring a minimum level of $30 \%$ fly ash. The expected 2 year results for both the virgin and recycled aggregates shows that the expansion of the mixes containing $25 \%$ FA HA-LC exceed the $0.04 \%$ limit, as expected, since 
Table 5.3.1 shows that a minimum of $30 \%$ should be used. The FA-HC used contains $26.41 \% \mathrm{CaO}$ and $2.11 \%$ alkalis, shown in Table 3.2.2, thus the required amount of fly ash is undetermined, however it is known that a minimum of $30 \%$ is necessary for moderate-calcium fly ashes $(8 \%$ $20 \% \mathrm{CaO}$ ). The $\mathrm{CPT}$ showed that $30 \% \mathrm{FA}-\mathrm{HC}$ was just enough to reduce the expected 2-year expansion of the virgin aggregate below $0.04 \%$, while $50 \%$ was required for the RCA. The slag, which contained a $1.10 \%$ alkali content showed that only $30 \%$ was required for the virgin aggregate and $40 \%$ was required for the RCA, despite the fact that the chart suggested a minimum use of $50 \%$. In addition, the HSF, which contained $8 \%$ silica fume, was enough to reduce the 2year expected expansion below $0.04 \%$ for both aggregates, even though a minimum of $10.7 \%$ is suggested.

When multiple SCM were used, each mix consistently contained $8 \%$ silica fume, which works out to three-quarters of the required amount. Thus, a minimum of one-quarter of the other SCM used is required, which was the case in the mixes containing FA HA-LC and slag. Therefore, only $7.5 \%$ FA HA-LC is required (where $25 \%$ was actually used) and $12.5 \%$ slag is required (where 30\% was used) when used with $8 \%$ silica fume. It is unknown what the required amount is for that of the FA-HC, but as explained above, 30\% was enough for the virgin aggregate and 50\% was enough for the RCA. Therefore when used with $8 \%$ silica fume, $12.5 \%$ should be enough for both aggregates (20\% and $30 \%$ were used). In all cases, except for the RCA mix containing HSF with $25 \%$ FA HA-LC, the two year expected expansion results satisfy the expectations laid out by Table 5.3.1.

\subsection{Comparing CPT Results of Sudbury Aggregates to Spratt Aggregates}

The results obtained using the concrete prisms test for the Sudbury aggregates shows that even the least effective SCM, 30\% FA-HC for instance, has proven to significantly reduce expansion. When 
looking at the 65-week summary of results of the CPT, shown in Figure 4.1.31, a drastic reduction in expansion occurs with the introduction of any SCM introduced. This coincides with previous studies, such as the work completed by Shehata et al. in 2010 on virgin Spratt aggregate and Spratt RCA. For example, the virgin Spratt aggregate and Spratt RCA both expand far beyond the $0.04 \%$ limit at 1 year, with the virgin aggregate expanding about $0.22 \%$ and the Spratt RCA expanding about $0.25 \%$. When SCM were introduced, $8 \%$ silica fume reduced the expansion in both Spratt aggregates to about $0.08 \%$ while $50 \%$ slag was enough to reduce the virgin Spratt aggregate to about $0.02 \%$ and the Spratt RCA to about $0.06 \%$. A comparison of the results obtained for Sudbury aggregates in this research and the results of the Spratt aggregates from Shehata et al. (2010) can be seen in Table 5.4.1.

Table 5.4.1: Comparing 1-year and expected 2-year expansion data for CPT of Sudbury and Spratt aggregates

\begin{tabular}{|c|c|c|c|c|}
\hline \multirow{2}{*}{ Mix Type } & \multicolumn{2}{|c|}{$\begin{array}{c}\text { 1-year and expected 2-year* } \\
\text { expansion value (\%) }\end{array}$} & \multicolumn{2}{|c|}{$\begin{array}{c}* * \text { 1-year and 2-year expansion value } \\
\text { obtained from Shehata et al. 2010 (\%) }\end{array}$} \\
\cline { 2 - 5 } & $\begin{array}{c}\text { Virgin } \\
\text { Sudbury } \\
\text { Aggregate }\end{array}$ & $\begin{array}{c}\text { Sudbury } \\
\text { RCA-M }\end{array}$ & $\begin{array}{c}\text { Virgin Spratt } \\
\text { Aggregate }\end{array}$ & Spratt RCA \\
\hline Control & 0.168 & 0.208 & 0.22 & 0.25 \\
\hline $\begin{array}{c}30 \% \text { High } \\
\text { calcium fly ash }\end{array}$ & $0.042^{*}$ & $0.070^{*}$ & 0.14 & 0.17 \\
\hline $\begin{array}{c}50 \% \text { High } \\
\text { calcium fly ash }\end{array}$ & $0.025^{*}$ & $0.042^{*}$ & & 0.11 \\
\hline $\begin{array}{c}30 \% \text { Slag } \\
\text { 50\% Slag }\end{array}$ & $0.027^{*}$ & $0.046^{*}$ & 0.02 & 0.06 \\
\hline $8 \%$ Silica Fume & $0.031^{*}$ & $0.042^{*}$ & 0.08 & 0.08 \\
\hline $\begin{array}{c}\text { 8\% Silica Fume } \\
\text { and 30\% FA-HC } \\
* * * \text { 5\% SF for }\end{array}$ & $0.014^{*}$ & $0.033^{*}$ & 0.03 & 0.07 \\
\hline Spratt & & & & 0.03 \\
\hline
\end{tabular}




\begin{tabular}{|c|c|c|c|c|}
\hline $\begin{array}{c}8 \% \text { Silica Fume } \\
\text { and 20\% FA-HC } \\
* * * \text { 5\% SF for } \\
\text { Spratt }\end{array}$ & $0.005 *$ & $0.026^{*}$ & 0.04 & 0.06 \\
\hline $\begin{array}{c}8 \% \text { Silica Fume } \\
\text { and 30\% Slag } \\
* * * 5 \% \text { SF for } \\
\text { Spratt }\end{array}$ & $0.022 *$ & $0.032 *$ & 0.01 & 0.06 \\
\hline
\end{tabular}

*Denotes 2-year expansion value is expected through linear interpolation of last 4 data points

$* *$ Values are estimated from graphs provided by Shehata et al. (2010)

***Denotes HSF containing $8 \%$ silica fume was used for Sudbury aggregates while only 5\% silica fume was used for Spratt aggregates

As shown in Table 5.4.1, more SCM is required to mitigate the expansion in concrete with Spratt aggregates due to its increased reactivity in comparison to the Sudbury aggregates. Aside from using 50\% slag, ternary blends of silica fume and either fly ash or slag were required to reduce expansion of the virgin Spratt aggregate. None of the mixes shown in Table 5.4.1 were effective in reducing the expansion of Spratt RCA below the $0.04 \%$ limit. Similar results between the aggregates were achieved when large amounts of replacement were used and with the use of ternary blends. However, when smaller amounts were used, such as $30 \%$ high calcium fly ash or $30 \%$ slag, the expansion in the samples containing Sudbury aggregates reduce significantly less than that of the Spratt samples. This is likely due to the change in expansion based on the level of $\mathrm{Na}_{2} \mathrm{O}_{\mathrm{e}}$ for each aggregate type, in which samples of Sudbury aggregate containing $0.8 \%$ or less $\mathrm{Na}_{2} \mathrm{O}_{\mathrm{e}}$ Portland cement expand less than $0.04 \%$ at 1 year while Spratt aggregate requires less than $0.7 \%$ (Shehata and Thomas, 2010). Additionally, at $1.0 \% \mathrm{Na}_{2} \mathrm{O}_{\mathrm{e}}$, Sudbury aggregate only expands about $0.12 \%$ compared to Spratt aggregate at about $0.22 \%$. Thus, less reduction of $\mathrm{Na}_{2} \mathrm{O}_{\mathrm{e}}$ is required for samples containing Sudbury aggregate. Figure 5.1.1, obtained from Shehata and Thomas (2010) shows the effects of the Portland cement alkali content on Spratt, Sudbury, and a third reactive sand (Jobe). The chart is assuming a control mix, thus no SCM are present. In the 
mixes completed in this research, sodium hydroxide $(\mathrm{NaOH})$ was used to bring the alkali content of the cement up to $1.25 \%$, thus providing an expansion above $0.15 \%$, as verified by Figure 5.1.1. When SCM was added to a mix, the alkali content of just the Portland cement was brought to $1.25 \%$. For example, a virgin Sudbury aggregate control mix has $100 \%$ of its cementing materials as GU Portland cement raised to $1.25 \% \mathrm{Na}_{2} \mathrm{O}_{\mathrm{e}}$. On the contrary, the mic containing $50 \%$ slag contained half the amount of cement, thus only half of the cementing materials were raised to $1.25 \% \mathrm{Na}_{2} \mathrm{O}_{\mathrm{e}}$. According to the Figure 5.1.1, a control mix with a $1.2 \%$ alkali content would expand roughly $0.16 \%$. The mix containing $50 \%$ slag only has half of its cement content raised to $1.25 \%$, so if the mix contains an alkali content below $1.0 \%$, a drastic reduction in expansion is expected, as shown in Figure 5.1.1. If the same was done to a mix containing virgin Spratt aggregate, less than $0.9 \%$ alkalis would be required before a drastic change in expansion would occur, thus a reduced alkali content and the use of SCM are more effective in mitigating expansion of Sudbury aggregate rather than Spratt aggregate, especially SCM with low alkali contents.

\subsection{Analyzing the Effects of Cement Content, Silane-sealer, and Sample Geometry}

The task 2 lab data provides a variety of comparisons, such as the effects of the cement content, the effects of a silane-based sealer, and the effects of the geometry of the sample. Firstly, shown in Figures 4.2.1 to 4.2.7, the expansion of the bridge mixes (containing $360 \mathrm{~kg} / \mathrm{m}^{3}$ ) is consistently less than that of the standard mixes (containing $420 \mathrm{~kg} / \mathrm{m}^{3}$ ). That being said, the difference in expansion is not very significant, but it is believed that the original bridge was cast with $360 \mathrm{~kg} / \mathrm{m}^{3}$, therefore the expansion obtained in those samples are used to compare to the cores and bridge barriers.

The silane-based sealer was used on all lab samples and was effective in reducing damages in all cases. Two coats of the sealer was applied before the samples underwent testing and was not 
reapplied throughout the 52-week duration. Although expansion was reduced with the sealer, the effects were minimal in comparison to the effects of SCM, shown in Figure 4.2.16. The sealer was just as effective on the extracted cores, thus it is suggested that the silane-based sealer is only used on existing structures as a technique to reduce the rate or slow down the expansion and damage. The silane-based sealer had proven to be more effective on the cylinders over than the prisms, likely due to their smooth surface and the lack of edges that are present in the prisms. Further testing should be completed on the effects of the sealer with multiple applications as well as the combined effect of SCM and the silane-based sealer.

The geometry and cross-section of the sample being measured has proven to have an effect on the expansion due to ASR. Testing was completed by the U.S. Department of Transportation in 2010 on the difference in expansion between cylinders and prisms which concluded that rate of expansion in cylinders of both $150 \mathrm{~mm}$ and $200 \mathrm{~mm}$ diameter were larger than that of concrete prisms of the same aggregate. The study suggests that cylinders of $150 \mathrm{~mm}$ in diameter or larger reduce alkali leaching (U.S. Department of Transportation 2010). The results obtained in task 2 between the cylinders and prisms confirm this. The cylinders consistently expanded at a higher rate than the prisms containing the same mix design due to the larger cross-sectional area, shown in Figures 4.2.10 and 4.2.11. This is because with a larger cross-section, there are less alkalis leaching from the sample. Therefore, there are more alkalis contained in the sample to react, causing an increase in the expansion of the cylindrical samples. 


\subsection{Analyzing the Expansion in Extracted Cores and Road Barriers}

The extracted cores were tested in the lab to determine to determine the level of residual expansion that would occur. Shown in Figure 4.2.12, there was residual expansion in both high and low deteriorated cores. However, the low deteriorated cores expanded at a far higher rate, with the 1year expansion being nearly double that of the high deteriorated cores. This could be due to three reasons: (1) the aggregate in the high deteriorated cores have little or no reactive silica or reactive sites remaining, (2) there are major cracks within the high deteriorated cores that allow the gel produced from ASR to divert to, thus causing slower expansion, and/or (3) alkalis were leached from cracked concrete. As the expansion curves reach week 52, shown in Figure 4.2.9, it is clear that the curves are beginning to level off, suggesting that the alkalis in the sample are depleting. Therefore, there is likely very little expansion that will continue to occur after the year-1 data. Figure 4.2.12 also shows that this residual expansion occurring in the cores is far less than that of the cylinders cast with RCA obtained from the same road barriers. This is because the majority of the expansion is occurring at the newly crushed faces of the aggregate in the RCA. Therefore, the cylinders that were cast with RCA are expanding due to the old aggregate-cement interface in addition to the newly formed interface of the new concrete, whereas the cores are only relying on the expansion of the old aggregate-cement bonds.

When looking at the expansion of the road barriers, shown in Figure 4.2.13, after 1 year the expansion results between the high and low deteriorated road barriers are almost identical. At the 1 year and 6 month reading, the reading was not taken at the same temperature as the zero reading and the 1 -year reading of $1^{\circ} \mathrm{C}$. Therefore, the equation for linear thermal expansion was used with an average coefficient of $12.2 \times 10^{6}\left({ }^{\circ} \mathrm{C}\right)$ (Neville, 2011). It is uncertain as to the accuracy of the coefficient, thus readings should be taken every 6 months, with the yearly interval reading 
taken at the same $1^{\circ} \mathrm{C}$ temperature. Once 3 or 4 years of readings are taken, the equation for linear expansion can be used to determine the actual coefficient of thermal expansion for the road barriers. This can be done by using the expansion data obtained yearly at the same temperature and fixing the readings taken at different temperatures to fit the curve, which would ignore thermal expansion. By determining that expansion value, and its original reading taken at a different temperature, the equation for linear expansion can be rearranged to determine the coefficient of linear expansion. In doing so, the readings thereafter can be taken at any temperature and corrected accordingly. The expansion of the road barriers will continue to be monitored for years to come in order to draw a correlation to lab and field data, as the expansion of structures in the field are far slower than samples in the lab, as shown in Figure 4.2.14.

\subsection{Analyzing the Expansion between RCA-M, RCA-H, and RCA-L}

The 1-year expansion results of the three RCA batches, shown in Figure 4.2.15, shows that all three batches provided almost identical expansion results. Therefore, it can be concluded that the level of deterioration that has occurred in the previous structure does not significantly affect the level of expansion that will occur in the new structure. This is likely the case due to the expansion occurring at the newly crushed faces in the RCA rather than a continuation of expansion of the previously exposed faces in the original concrete. Figure 4.2.12 helps solidify this assumption as is it shows that the level of expansion occurring in the new concrete is far higher than that of the expansion occurring in the cores. Although the high deteriorated cores expand at half the rate of the low deteriorated cores, the expansion of both are so minimal, that the difference between them has little to no affect.

It is important to note that all tests were completed using either $100 \%$ reactive virgin aggregate or $100 \%$ reactive RCA. In general, RCA is used as partial replacement of the natural 
aggregates in concrete. This is because using the whole content of coarse aggregate as RCA produces concrete of low workability, high shrinkage and perhaps lower strength. An earlier study (Shehata et al. 2012) showed that when blending reactive RCA with non-reactive coarse aggregate, the expansion is reduced. A number of tests were completed and showed that $100 \%$ reactive RCA yielded over $0.20 \%$ expansion at year 2 whereas $70 \%$ RCA with $30 \%$ non-reactive dolomite yielded just over $0.10 \%$ expansion at year 2 (Shehata et al. 2012). Furthermore, when adding $25 \%$ low-calcium fly ash or 50\% slag, the year 2 expansion was below $0.04 \%$, the maximum accepted value (Shehata et al. 2012). Therefore it is reasonable to assume that the same could be achieved using a portion of Sudbury RCA investigated here and non-reactive aggregate. It should be noted that the common level of RCA used in concrete ranges from $20 \%$ to $40 \%$. At that level, it is anticipated that the expansion can be mitigated using moderate level of preventive measures. 


\section{Chapter 6: Conclusions}

The purpose of this research was to determine the usability of RCA obtained from structures affected by previous deterioration mechanisms, namely Alkali-silica reaction. In this case, accelerated lab testing on RCA originally cast with the moderately alkali-reactive Sudbury aggregate, previously affected by ASR, was compared to samples cast with the virgin Sudbury aggregate as well as extracted cores from the original structure. Field tests were also completed on the original road barriers to compare the accelerated lab tests. A summary of the findings are presented below.

1. Concrete containing alkali reactive RCA produces higher expansion than the original virgin aggregate used in the RCA.

2. Reactive RCA requires higher levels of SCM to mitigate the expansion compared to the virgin reactive aggregate.

3. The concrete microbar test produces similar expansion trends to that of the concrete prism test. In both tests, samples containing Sudbury RCA consistently expanded at higher rates than the same samples containing virgin Sudbury aggregate, likely due to the residual expansion occurring in the previous aggregate-cement bonds. It is suggested that the CMBT 28-day limit of $0.04 \%$ is not appropriate for Sudbury aggregate with SCM. Instead, a 28 -day limit of $0.10 \%$ is believed to be more appropriate. However, this observation needs to be verified when the two-year results of the CPT are available.

4. The accelerated microbar test does not provide similar trends between RCA and the virgin aggregate, suggesting that the crushing of the aggregate to fines breaks apart the part of the originally formed gel, reducing the expansion. 
5. The level of deterioration that has previously affected the structure containing gravel reactive aggregate does not have a significant effect on its reactivity as an RCA. This suggests that the reaction occurs, mainly, at the newly crushed faces of the aggregate.

6. With the use of moderate levels of SCM, RCA can be safely used as partial replacement of the coarse aggregate in concrete. Testing should be carried out to identify the type and levels of SCM required with the use of partial RCA replacements.

7. Concrete cylinders expand at a higher rate than concrete prisms of the same mix design, which is likely a result of the decrease in alkali leaching in the cylindrical samples.

8. Reducing the cement content of the mix design results in a decrease in expansion.

9. The expansion of the low deteriorated cores is much higher than that of the high deteriorated cores. This suggests that one or a combination of the following is taken place (1) after a certain amount of expansion has occurred, ASR begins to slow down and/or stop, (2) cracking is so severe in the high deteriorated cores that the expansive gel is able to navigate within the cracks and show less expansion, or (3) the cracking promoted more alkali leaching from the core samples.

10. Silane-based sealers can be used to reduce expansion due to ASR, however SCM are far more effective. Sealers should only be used on existing structures as a mitigation technique and further testing should be completed on the combined mitigating effects of SCM and the sealer. 
11. The expansion measured on the road barriers left on site was much slower than the samples tested under accelerated conditions in the lab. Further testing is required to compare lab and field data. 


\section{Chapter 7: Recommendations for Future work}

- Concrete prisms samples containing the same low-alkali, low-calcium fly ash in the microbar and mortar bar samples should be completed in order to verify its effectiveness. The prisms samples were cast with a high-alkali, low-calcium fly ash which provided larger expansion results.

- Samples should be cast with partial RCA replacements ranging between $20 \%$ and $40 \%$ in addition to a non-reactive aggregate for the accelerated mortar bar test, concrete microbar test, and concrete prisms test. The samples tested in this research are cast with $100 \%$ RCA replacement, which is not realistic for use in construction. In using a 20$40 \%$ partial replacement, the exact levels and types of SCM required can be determine for use in construction.

- Samples should be cast of cylinders and prisms to test the effects of the silane-based sealer with multiple applications. The samples tested in this research only included an initial sealer application, prior to testing. Samples can be tested with sealer application every $4,8,16$, and 32 weeks to determine the effectiveness of multiple applications.

- Samples should be cast with varying levels of SCM in addition to the silane-based sealer to determine the combined mitigating effects. In doing so, less SCM may be required to mitigate ASR expansion. 


\section{References}

Adams, M.P., Jones, A., Beauchemin, S., Johnson, R., Fournier, B., Shehata, M., Tanner, J.E., Ideker, J.H. (2013). Applicability of the Accelerated Mortar Bar Test for Alkali-Silica Reactivity of Recycled Concrete Aggregates. ASTM International, 2(1) ACEM20120030.

ASTM International. (2011). Standard Practice for Use of Apparatus for the Determination of Length Change of Hardened Cement Paste, Mortar, and Concrete. ASTM C490, Pennsylvania, United States.

ASTM International. (2012). Standard Test Method for Scaling Resistance of Concrete Surfaces Exposed to Deicing Chemicals. ASTM C672, Pennsylvania, United States.

ASTM International. (2014). Standard Practice for Mechanical Mixing of Hydraulic Cement Pastes and Mortars of Plastic Consistency. ASTM C305, Pennsylvania, United States.

ASTM International. (2014). Standard Test Method for Potential Alkali Reactivity of Aggregates (Mortar-Bar Method). ASTM C1260, Pennsylvania, United States.

ASTM International. (2015). Standard Practice for Making and Curing Concrete Test Specimens in the Laboratory. ASTM C192, Pennsylvania, United States.

ASTM International. (2015). Standard Test Method for Relative Density (Specific Gravity) and Absorption of Coarse Aggregate. ASTM C127, Pennsylvania, United States.

ASTM International. (2015). Standard Test Method for Relative Density (Specific Gravity) and Absorption of Fine Aggregate. ASTM C128, Pennsylvania, United States.

ASTM International. (2015). Standard Test Method for Resistance of Concrete to Rapid Freezing and Thawing. ASTM C666, Pennsylvania, United States.

Canadian Standards Association. (2009). Concrete materials and methods of concrete construction. CSA A23.1-09, Ontario, Canada. 
Canadian Standards Association. (2009). Potential expansivitiy of aggregates (procedure for length change due to alkali aggregate reaction in concrete prisms at $38 \mathrm{C}$ ). CSA A23.214A, Ontario, Canada.

Canadian Standards Association. (2009). Standard practice for laboratory testing to demonstrate the effectiveness of supplementary cementing materials and lithium-based admixtures to prevent alkali-silica reaction in concrete. CSA A23.2-28A, Ontario, Canada.

Canadian Standards Association. (2009). Standard practice to identify degree of alkali- aggregate reactivity of aggregates and to identify measures to avoid deleterious expansion in concrete. CSA A23.2-27A, Ontario, Canada.

Canadian Standards Association. (2009). Test method for detection of alkali-silica reactive aggregate by accelerated expansion of mortar bars. CSA A23.2-25A, Ontario, Canada.

Diamond, S. (1983). Alkali reactions in concrete - Pore solution effects. Proceedings of the 6th International Conference on Alkalis in Concrete, (Ed. G.M. Idorn and Steen Rostam), Danish Concrete Association, Copenhagen, 155-166.

Goguen, C. (2012). Air Entrainment versus Air Entrapment. Retrieved August 9, 2014, from http://precast.org/2012/12/air-entrainment-versus-air-entrapment/

Grattan-Bellew, P.E., Du-you, L., Fournier, B., Mitchell, L. (2004). Comparison of expansions in the concrete prism and concrete microbar tests of an assorted suite of aggregates from several countries. Proceedings of the 12th International Conference on Alkali-Aggregate Reaction in Concrete, Beijing, China, 251-256.

Hodgson, S. S. (2000). The Effects of Water/Cement Ratio and Air Entraining on Portland Cement Concrete Freeze/Thaw Durability. University of Wisconsin-Madison, Environmental Engineering Department. Retrieved August 13, 2014, from http://www.hottmannconstruction.com/Paper.PDF.

Lothenbach, B., Scrivener, K., Hooton, R.D. (2011). Supplementary cementitious materials. Cement and Concrete Research 41(3), 1244-1256. 
Mamlouk, Michael S. and Zaniewski, John P. (1999). Materials for Civil and Construction Engineers. Prentice Hall, Pearson.

National Ready Mix Concrete Association. (1998). Scaling Concrete Surfaces. Retrieved August 8, 2014, from http://www.nrmca.org/aboutconcrete/cips/02p.pdf

Neville, A.M. (2011). Properties of Concrete, Fifth Edition. Pearson.

Portland Cement Association. (2014). Freeze-Thaw Resistance. Retrieved August 10, 2014, from http://www.cement.org/for-concrete-books-learning/concretetechnology/durability/freeze-thaw-resistance

Radonjanin, V., Malesev, M., Marinkovic, S., Malty, A. (2013). Green recycled aggregate concrete. Construction and Building Materials.

Ronning, T. F. (2001). Freeze-Thaw Resistance of Concrete Effect of: Curing Conditions, Moisture Exchange and Materials. The Norwegian Institute of Technology, Division of Structural Engineering, Concrete Section.

Salcedo, A. (1984). Identification of Frost Susceptible Aggregate and Their Use in Concrete or Bituminous Pavements. Indiana Department of Highways, School of Civil Engineering.

Seymour. (2014). Roberts-Seymour Consulting: Freeze Thaw Damage to Portland Cement Steel Reinforced Concrete. Retrieved August 16, 2014, from http://www.roberts-seymourgroup.com/2012/04/freeze-thaw-damage-to-portland-cement.html

Shang, H., Cao, W., \& Wang, B. (2014). Effect of Fast Freeze-Thaw Cycles on Mechanical Properties of Ordinary-Air-Entrained Concrete. Retrieved August 16, 2014, from http://www.hindawi.com/journals/tswj/2014/923032/

Shehata, M., Christidis, C., Mikhaiel, W., Rogers, C., Lachemi, M. (2010). Reactivity of reclaimed concrete aggregate produced from concrete affected by alkali-silica reaction. Cement and Concrete Research 40(4), 575-582.

Shehata M.H., Mikhaeel W, Lachemi M, Rogers, C. (2012). Preventive Measures against Expansion in Concrete Containing Reactive Recycled Concrete Aggregate. Texas: 
Proceedings of the $14^{\text {th }}$ International Conference on Alkali Aggregate Reaction in Concrete.

Shehata, M.H., Thomas, M.D.A. (2000). The effect of fly ash composition on the expansion of concrete due to alkali-silica reaction. Cement and Concrete Research 30(7), 1063-1072.

Shehata, M.H., Thomas, M.D.A. (2010). The role of alkali content of Portland cement on the expansion of concrete prisms containing reactive aggregates and supplementary cementing materials. Cement and Concrete Research, 40(4), 569-574.

Siddique, R. and Khan, M.I. (2011). Supplementary Cementing Materials. Engineering Materials, 37.

Sika. (2014). Frost and Freeze / Thaw Resistant Concrete. Retrieved August 12, 2014, from http://www.sika.com/en/concrete-redirect/sika-concrete-technology/concrete-handbook2013/concrete-types/thaw-resistant-concrete.html

Sommer, H., Nixon, P.J., Sims, I. (2005). AAR-5: Rapid preliminary screening test for carbonate aggregates. Rilem, Materials and Structures 38, 787-792.

Stanton, T.E. (1940). Expansion of concrete through reaction between cement and aggregate. Proceedings of the American Society of Civil Engineers, 66(10), 1781-1811.

Supplementary Cementitious Materials. (2006). Texas Contractor, 154(12). Retrieved from http://search.proquest.com.ezproxy.lib.ryerson.ca/docview/196612119/fulltext/D7963984 97544773PQ/1?accountid=13631.

Thomas, M.D.A., Fournier, B., Folliard, K., Ideker, J., Shehata, M. (2006). Test methods for evaluating preventative measures for controlling expansion due to alkali-silica reaction in concrete. Cement and Concrete Research 36(10), 1842-1856.

Thomas, M.D.A., Fournier, B., Folliard, K.J. (2013). Alkali-Aggregate Reactivity (AAR) Facts Book. U.S. Department of Transportation: Federal Highway Administration. 